Supporting Information for:

\title{
Modeling the Catalytic Cycle of Glutathione Peroxidase by Nuclear Magnetic Resonance Spectroscopic Analysis of Selenocysteine Selenenic Acids
}

Ryosuke Masuda, Ryutaro Kimura, Takafumi Karasaki, Shohei Sase and Kei Goto*

Department of Chemistry, School of Science, Tokyo Institute of Technology, 2-12-1 Ookayama, Meguro-ku, Tokyo 152-8551, Japan

\section{Contents}

1. Experimental section $\quad$ S2

2. NMR spectra $\quad$ S36

3. References $\quad \mathbf{S 6 5}$ 


\section{General experimental section}

THF (anhydrous) and $\mathrm{Et}_{2} \mathrm{O}$ (anhydrous) were purchased from Kanto Chemical and passed through a Kayama Oxygen solvent purification system prior to use. 1,4-dioxane, dichloromethane, and toluene were purchased from commercial sources and dried over $4 \AA \mathrm{MS}$. $\mathrm{CDCl}_{3}$ was passed through a small column of neutral alumina prior to use. $\mathrm{Et}_{3} \mathrm{~N}$ and ${ }^{i} \mathrm{Pr}_{2} \mathrm{NEt}$ were purchased from commercial sources and distilled over $\mathrm{KOH}$ and then $\mathrm{CaH}_{2}$. Other chemicals were purchased from commercial sources and used as received. Silica gel column chromatography was performed using Kanto silica gel N60 or N40-50. Preparative thin layer chromatography (PTLC) was performed using Merck silica gel 60 PF254. Preparative gel permeation liquid chromatography (GPLC) was performed by LC9210 NEXT with JAI gel 2HR and 2.5HR columns (Japan Analytical Industry) with chloroform as solvent. ${ }^{1} \mathrm{H}$ NMR spectra were recorded on a JEOL ECX-500, a JEOL ECZ-500, a JEOL ECS-400 and the chemical shifts of ${ }^{1} \mathrm{H}$ are referenced to the residual proton signal of $\mathrm{CDCl}_{3}\left(\delta\right.$ 7.25), $\mathrm{CD}_{3} \mathrm{OD}(\delta 3.31)$, and THF-d $d_{8}(1.72) .{ }^{13} \mathrm{C}\left\{{ }^{1} \mathrm{H}\right\} \mathrm{NMR}$ spectra were recorded on a JEOL ECX-500 or a JEOL ECZ-500, and the chemical shifts are given relative to $\mathrm{CDCl}_{3}$ $(\delta 77.23)$ and $\mathrm{CD}_{3} \mathrm{OD}(\delta 49.00)$ as internal standards. ${ }^{77} \mathrm{Se}\left\{{ }^{1} \mathrm{H}\right\}$ NMR spectra were recorded on a JEOL ECX-500, and the chemical shifts of ${ }^{77} \mathrm{Se}$ are referenced to $\mathrm{Ph}_{2} \mathrm{Se}_{2}(\delta 480)$ as an external standard. All spectra were assigned with the aid of DEPT, COSY, HMQC, and HMBC NMR experiments. IR spectra were recorded on a JASCO FT/IR4100. UV-vis spectra were recorded on a JASCO V-650 UV-vis spectrometer. High-resolution FD-TOF mass spectra were measured on a JEOL JMS-T100GCv “AccuTOF GCv". High-resolution FAB mass spectra were measured on a JEOL JMS-700 MStation mass spectrometer. High-resolution ESI-TOF mass spectra were measured on a Bruker "micrOTOF II" at Open Facility Center in Tokyo Institute of Technology. Melting points (m.p.) were measured with a Yanaco MP-S3 (uncorrected).

\section{Synthesis of BpscOH (S3) with a procedure modified from the previous report. ${ }^{2}$}

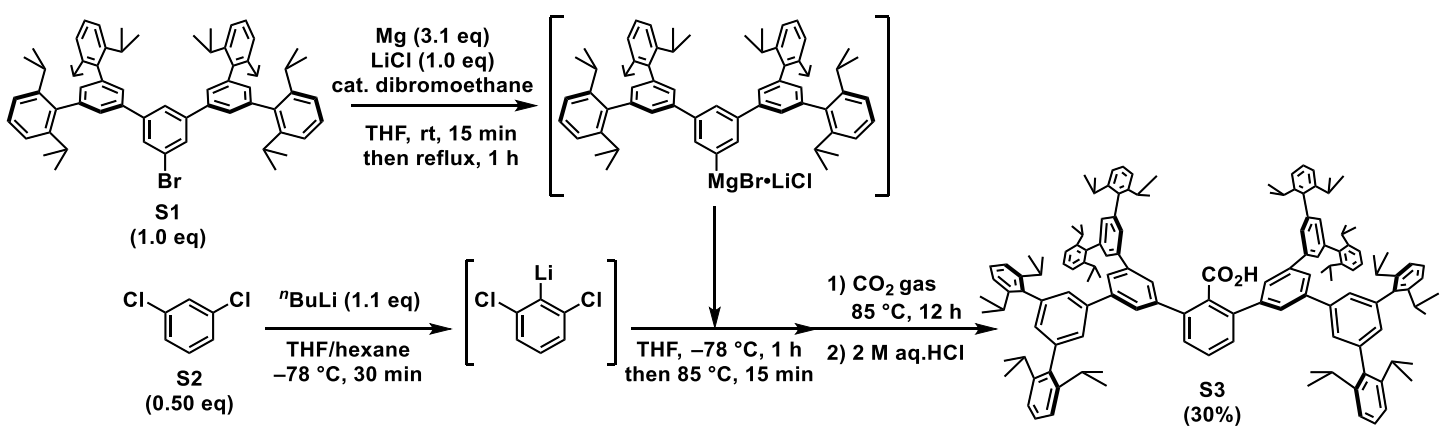

Scheme S1. Synthesis of a framework of Bpsc by utilizing a Grignard reagent.

A two-necked $200 \mathrm{~mL}$ round-bottom flask, which was equipped with a magnetic stir bar and charged with magnesium turnings $(1.37 \mathrm{~g}, 56.6 \mathrm{mmol})$ and $\mathrm{LiCl}(851 \mathrm{mg}, 20.1 \mathrm{mmol})$, was fitted with a reflux condenser, a glass stopper, and a rubber septum. The flask was heated and dried under vacuum, evacuated and backfilled with argon. Aryl bromide $\mathbf{S 1}(17.4 \mathrm{~g}, 18.3 \mathrm{mmol})^{1}$ in THF $39 \mathrm{~mL}$ was slowly added. The reaction mixture was heated to $90{ }^{\circ} \mathrm{C}$ and 1,2-dibromoethane $(9 \mu \mathrm{L})$ was added. The reaction mixture was allowed to stir at $90{ }^{\circ} \mathrm{C}$ for $1 \mathrm{~h}$ and then cooled to room temperature. A separate two-necked $200 \mathrm{~mL}$ round-bottom flask was equipped with a magnetic stir bar, fitted with a rubber septum and a reflux condenser. It was heated and dried under vacuum, evacuated and backfilled with argon. THF (13 mL) and freshly distilled 1,3-dichlorobenzene (S2; $1.04 \mathrm{~mL}, 9.13 \mathrm{mmol})$ were added to the 
flask. The reaction mixture was cooled to $-78{ }^{\circ} \mathrm{C}$ and ${ }^{n} \mathrm{BuLi}$ (1.59 $\mathrm{M}$ in hexane, $5.90 \mathrm{~mL}, 9.31 \mathrm{mmol}$ ) was added by a syringe pump over $30 \mathrm{~min}$. The solution was stirred for an additional $40 \mathrm{~min}$ and the Grignard reagent, which was prepared in the first reaction vessel, was added through a Teflon tube over a 20 min period. The resulting mixture was allowed to stir at $-78^{\circ} \mathrm{C}$ for $20 \mathrm{~min}$ and then slowly warmed to room temperature over $30 \mathrm{~min}$. After it was stirred for an additional $9 \mathrm{~h}$ at $85{ }^{\circ} \mathrm{C}$, the mixture was cooled to room temperature and the vessel was filled with $\mathrm{CO}_{2}$ gas. The black solution was heated to $85^{\circ} \mathrm{C}$ for $5 \mathrm{~h}$. To the mixture, $2 \mathrm{M}$ aq. $\mathrm{HCl}(40 \mathrm{~mL})$ was added to quench on an ice bath. The two layers were separated and the aqueous layer was extracted with $\mathrm{CHCl}_{3}(3 \times 50 \mathrm{~mL})$. The combined organic layer was washed with brine, dried over $\mathrm{Na}_{2} \mathrm{SO}_{4}$, and filtered. The filtrate was concentrated in vacuo and the crude product was purified by flash column chromatography on silica gel (hexane $/ \mathrm{CHCl}_{3}=3: 1$

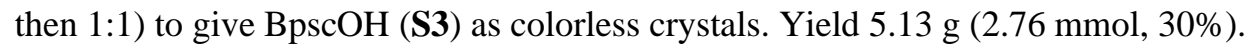

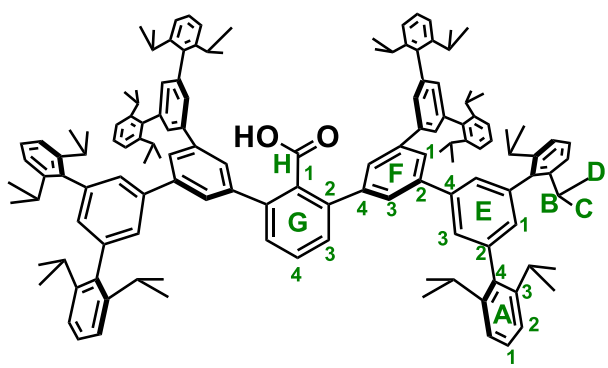

BpscOH (S3) ${ }^{2}$ : colorless crystals; m.p. $235.5-238.5^{\circ} \mathrm{C} .{ }^{1} \mathbf{H}$ NMR $(500$ $\left.\mathrm{MHz}, \mathrm{CDCl}_{3}\right): \delta 1.02(\mathrm{~d}, J=6.9 \mathrm{~Hz}, 48 \mathrm{H}, \mathbf{C}$ or $\mathbf{D}), 1.09(\mathrm{~d}, J=6.9 \mathrm{~Hz}$, $48 \mathrm{H}, \mathbf{C}$ or D), 2.73 (septet, $J=6.9 \mathrm{~Hz}, 16 \mathrm{H}, \mathbf{B}), 6.98(\mathrm{t}, J=1.4 \mathrm{~Hz}, 4 \mathrm{H}$, E1), $7.16(\mathrm{~d}, J=7.8 \mathrm{~Hz}, 16 \mathrm{H}, \mathbf{A 2}), 7.31(\mathrm{t}, J=7.8 \mathrm{~Hz}, 8 \mathrm{H}, \mathbf{A 1}), 7.42$ $(\mathrm{d}, J=1.4 \mathrm{~Hz}, 8 \mathrm{H}, \mathbf{E 3}), 7.48-7.52$ (m, 3H, G3 and G4), 7.72 (d, $J=1.8$ $\mathrm{Hz}, 4 \mathrm{H}, \mathbf{F 3}), 7.83$ (t, $J=1.8 \mathrm{~Hz}, 2 \mathrm{H}, \mathbf{F 1}) ;{ }^{13} \mathbf{C} \mathbf{~ N M R}\left(125 \mathrm{MHz}, \mathrm{CDCl}_{3}\right)$ : $\delta 24.3$ (q, C or D), 24.5 (q, C or D), 30.6 (d, B), 122.6 (d, A2), 125.8 (d, F1), 126.6 (d, E3), 126.9 (d, F3), 128.1 (d, A1), 129.4 (d, G3), 129.8 (d, G4), 130.2 (d, E1), 132.7 (s, G1), 139.2 (s, A4), 139.9 (s, G2), 140.6 (s, F2), 141.1 (s, E2), 141.4 (s, F4), 141.9 (s, E4), 146.9 (s, A3), 171.1 (s, H); IR (KBr); 3446 (br), 2961, 2927, 2867, 1732, 1716, 1577, 1457, 1362, 1325, 1250, 1179, 873, 828, 805, $754 \mathrm{~cm}^{-1}$. HRMS (FD-TOF) $m / z$ 1859.2222 [M] $]^{+}$(calcd for $\mathrm{C}_{139} \mathrm{H}_{158} \mathrm{O}_{2}, 1859.2261$ ).

\section{Synthesis of BpscCl (S4). ${ }^{2}$}

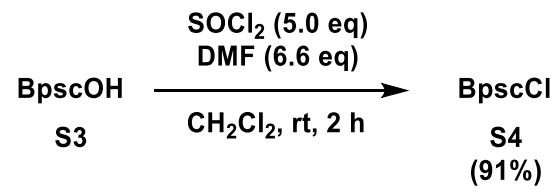

Scheme S2. Synthesis of acid chloride $\mathbf{S 4}$.

BpscOH (S3; $1.15 \mathrm{~g}, 0.616 \mathrm{mmol})$ was placed in a $25 \mathrm{~mL}$ two-necked flask. After evacuated and backfilled with argon, $\mathrm{CH}_{2} \mathrm{Cl}_{2}(15 \mathrm{~mL})$ and DMF $(0.29 \mathrm{~mL}, 3.7 \mathrm{mmol})$ were added and then $\mathrm{SOCl}_{2}(0.22 \mathrm{~mL}, 3.1 \mathrm{mmol})$ was slowly added. The reaction mixture was stirred at room temperature. After $2 \mathrm{~h}$, the reaction mixture was concentrated in vacuo. The residue was dissolved in $\mathrm{CH}_{2} \mathrm{Cl}_{2}$ and concentrated in vacuo for three times. The obtained solid was washed with $\mathrm{CH}_{3} \mathrm{CN}$ and dried under vacuum at $70{ }^{\circ} \mathrm{C}$ to give $\mathrm{BpscCl}(\mathbf{S 4})$ as colorless crystals. Yield $1.05 \mathrm{~g}(0.560$ mmol, 91\%). Addition of DMF was crucial to prevent intramolecular Friedel-Crafts type reaction.

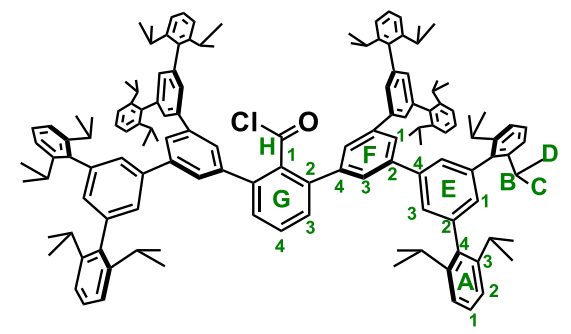

BpscCl (S4): colorless crystals; m.p. 248.9-250.5 ${ }^{\circ} \mathrm{C}$ (dec). ${ }^{1} \mathbf{H}$ NMR (500 $\left.\mathrm{MHz}, \mathrm{CDCl}_{3}\right): \delta 1.03(\mathrm{~d}, J=6.9 \mathrm{~Hz}, 48 \mathrm{H}, \mathbf{C}$ or $\mathbf{D}), 1.09(\mathrm{~d}, J=6.9 \mathrm{~Hz}, 48 \mathrm{H}$, C or D), 2.73 (septet, $J=6.9 \mathrm{~Hz}, 16 \mathrm{H}, \mathbf{B}), 6.99$ (t, $J=1.5 \mathrm{~Hz}, 4 \mathrm{H}, \mathbf{E 1}), 7.16$ (d, $J=7.8 \mathrm{~Hz}, 16 \mathrm{H}, \mathbf{A 2}), 7.31(\mathrm{t}, J=7.8 \mathrm{~Hz}, 8 \mathrm{H}, \mathbf{A 1}), 7.45(\mathrm{~d}, J=1.5 \mathrm{~Hz}$, $8 \mathrm{H}, \mathbf{E 3}), 7.50-7.52(\mathrm{~m}, 2 \mathrm{H}, \mathbf{G 3}), 7.56-7.59(\mathrm{~m}, 1 \mathrm{H}, \mathbf{G 4}), 7.68(\mathrm{~d}, J=1.5 \mathrm{~Hz}$, 
4H, F3), 7.90 (t, $J=1.5 \mathrm{~Hz}, 2 \mathrm{H}, \mathbf{F 1}) ;{ }^{13} \mathbf{C}$ NMR (125 MHz, $\left.\mathrm{CDCl}_{3}\right): \delta 24.3$ (q, C or D), 24.5 (q, C or D), 30.6 (d, B), 122.7 (d, A2), 126.2 (d, F1), 126.5 (d, E3), 127.1 (d, F3), 128.1 (d, A1), 129.7 (d, G3), 130.5 (d, E1), 130.9 (d, G4), 137.4 (s, G1), 139.14 (s, A4), 139.18 (s, G2), 140.0 (s, F2), 140.3 (s, F4), 141.3 (s, E2), 142.1 (s, E4), 146.9 (s, A3), 170.3 (s, H); IR (KBr); 3061, 3033, 2961, 2926, 2868, 1792, 1594, 1577, 1462, 1383, 1362, 1325, 1180, $1055,874,859,804,761,753,726,695,651 \mathrm{~cm}^{-1}$. HRMS (FD-TOF) $\mathrm{m} / \mathrm{z} .1877 .2004[\mathrm{M}]^{+}$(calcd for $\mathrm{C}_{139} \mathrm{H}_{157} \mathrm{ClO}$, 1877.1922).

\section{Synthesis of asymmetric selenocystine 2 .}

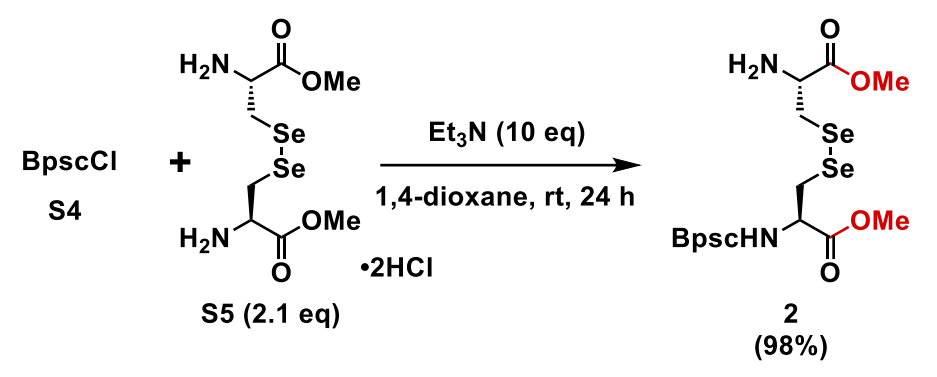

Scheme S3. Synthesis of asymmetric diselenide 2.

Freshly prepared L-selenocystine methyl ester dihydrochloride (S5; $112 \mathrm{mg}, 0.258 \mathrm{mmol}$ ), which was prepared by the reported procedure, ${ }^{3}$ was placed in a $25 \mathrm{~mL}$ Schlenk tube. After evacuated for $30 \mathrm{~min}$ and backfilled with argon, 1,4-dioxane (5.0 mL), BpscCl (S4; $237 \mathrm{mg}, 0.126 \mathrm{mmol})$ and then $\mathrm{Et}_{3} \mathrm{~N}(0.16 \mathrm{~mL}, 1.2 \mathrm{mmol})$ were added. The resulting reaction mixture was stirred at room temperature for $19 \mathrm{~h}$ before water was added. The two layers were separated and the aqueous layer was extracted with $\mathrm{CHCl}_{3}(3 \times 8 \mathrm{~mL})$. The combined organic layer was washed with brine, dried over $\mathrm{Na}_{2} \mathrm{SO}_{4}$, and filtered. The filtrate was concentrated in vacuo and the crude product was purified by flash column chromatography on silica gel (hexane/ $\mathrm{CHCl}_{3}=1: 7$ then 1:10) to give $\mathbf{2}$ as yellow crystals. Yield $272 \mathrm{mg}$ (0.123 mmol, 98\%).

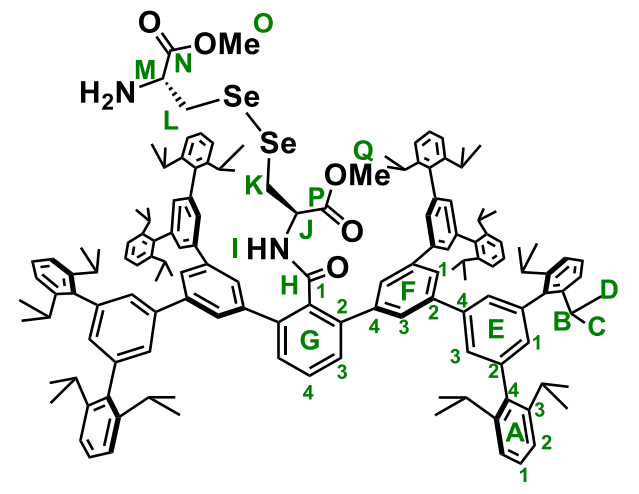

2: yellow crystals; m.p. $252-255{ }^{\circ} \mathrm{C} .{ }^{1} \mathbf{H}$ NMR $\left(500 \mathrm{MHz}, \mathrm{CDCl}_{3}\right): \delta$ 1.02-1.04 (m, 48H, C and D), 1.09-1.12 (m, 48H, C and D), 2.54 (dd, $J$ $\left.=13.1,4.9 \mathrm{~Hz}, 1 \mathrm{H}, \mathbf{K}_{\mathrm{A}}\right), 2.70-2.79(\mathrm{~m}, 16 \mathrm{H}, \mathbf{B}), 2.86(\mathrm{~d}, J=6.0 \mathrm{~Hz}, \mathbf{L})$, $2.92\left(\mathrm{dd}, J=13.1,4.2 \mathrm{~Hz}, 1 \mathrm{H}, \mathbf{K}_{\mathbf{B}}\right), 2.98(\mathrm{~s}, 3 \mathrm{H}, \mathbf{Q}), 3.36(\mathrm{t}, J=6.0 \mathrm{~Hz}$, $1 \mathrm{H}, \mathbf{M}), 3.50(\mathrm{~s}, 3 \mathrm{H}, \mathbf{O}), 4.63(\mathrm{dt}, J=7.8,4.5 \mathrm{~Hz}, 1 \mathrm{H}, \mathbf{J}), 6.59(\mathrm{~d}, J=$ $8.1 \mathrm{~Hz}, 1 \mathrm{H}, \mathbf{I}), 6.98$ (br, 4H, E1), 7.16-7.18 (m, 16H, A2), 7.31 (t, $J=$ 7.7 Hz, 8H, A1), 7.42-7.44 (m, 10H, E3 and G3), 7.47-7.50 (m, 1H, G4), 7.68 (br, 4H, F3), 7.78 (br, 2H, F1); $\left.{ }^{13} \mathbf{C ~ N M R ~ ( 1 2 5 ~ M H z , ~} \mathrm{CDCl}_{3}\right)$ :

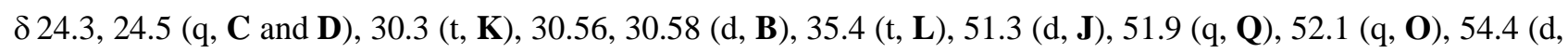
M), 122.6 (d, A2), 126.1 (d, F1), 126.7 (d, E3), 127.2 (d, F3), 128.0 (d, A1), 129.4 (d, G4), 130.0 (d, G3), 130.4 (d, E1), 134.8 (s, G1), 139.2 (s, A4), 140.2 (s, G2), 140.6 (s, F2), 141.1 (s, E2), 141.6 (s, F4), 141.8 (s, E4), 146.8 (s, A3), 168.0 (s, H), 170.0 (s, P), 173.9 (s, N); $\left.{ }^{77} \mathbf{S e ~ N M R ~ ( 9 5 ~ M H z , ~ C D C l ~}\right)$ ): $\delta 285,286$; IR (KBr); 3418 (br), 3390 , 3334, 3275, 3062, 3030, 3008, 2961, 2926, 2867, 1746, 1681, 1669, 1583, 1576, 1505, 1469, 1462, 1383, 1361, 1325, 1249, 1216, 1177, 1055, 873, 805, 754, $726 \mathrm{~cm}^{-1}$. HRMS (FD-TOF) $\mathrm{m} / \mathrm{z} 2205.1638[\mathrm{M}]^{+}$(alcd for $\mathrm{C}_{147} \mathrm{H}_{172} \mathrm{~N}_{2} \mathrm{O}_{5} \mathrm{Se}_{2}$, 2205.1596). Anal. Calcd. for $\mathrm{C}_{147} \mathrm{H}_{172} \mathrm{~N}_{2} \mathrm{O}_{5} \mathrm{Se}_{2}$ : C, 80.08; H, 7.86; N, 1.27. Found: C, 79.83; H, 


\section{Synthesis of selenol 3a.}

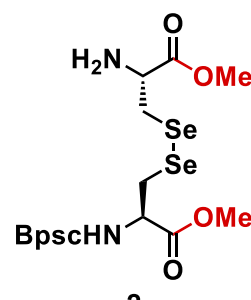

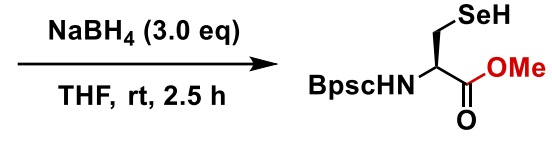

$3 a$

$(86 \%)$

Scheme S4. Synthesis of selenol 3a.

The following reaction and workup were performed under argon atmosphere. Asymmetric diselenide 2 (260 mg, $0.118 \mathrm{mmol})$ was placed in a $25 \mathrm{~mL} \mathrm{~J}$-young tube. After evacuated and backfilled with argon, THF (6.0 mL) and $\mathrm{NaBH}_{4}(13.4 \mathrm{mg}, 0.354 \mathrm{mmol})$ were added. The resulting reaction mixture was stirred at room temperature for 2.5 $\mathrm{h}$ before degassed water was added. The two layers were separated and the aqueous layer was extracted with degassed $\mathrm{CHCl}_{3}(3 \times 8 \mathrm{~mL})$ using Schlenk tubes. The combined organic layer was washed with degassed brine, dried over $\mathrm{Na}_{2} \mathrm{SO}_{4}$, and filtered through Celite. The filtrate was concentrated in vacuo to give $\mathbf{3 a}$ as colorless crystals. Yield $203 \mathrm{mg}(0.101 \mathrm{mmol}, 86 \%)$.

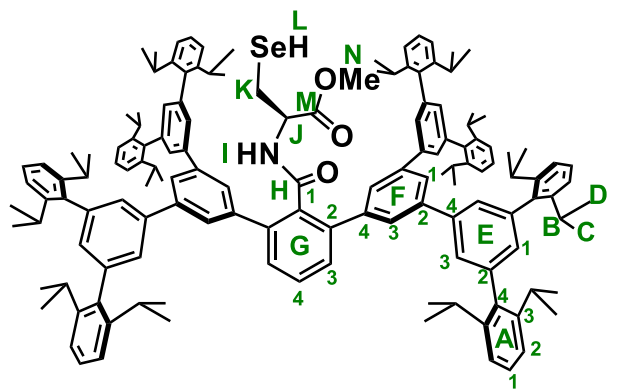

Selenol 3a: colorless crystals; m.p. $240-243{ }^{\circ} \mathrm{C} .{ }^{1} \mathbf{H}$ NMR $(500 \mathrm{MHz}$, $\left.\mathrm{CDCl}_{3}\right): \delta-1.52(\mathrm{t}, J=8.0 \mathrm{~Hz}, 1 \mathrm{H}, \mathbf{L}), 1.02-1.04(\mathrm{~m}, 48 \mathrm{H}, \mathbf{C}$ and $\mathbf{D})$, $1.08-1.14(\mathrm{~m}, 48 \mathrm{H}, \mathbf{C}$ and $\mathbf{D}), 2.22\left(\mathrm{ddd}, J=12.6,8.0,4.0 \mathrm{~Hz}, 1 \mathrm{H}, \mathbf{K}_{\mathbf{A}}\right)$, $2.50\left(\mathrm{ddd}, J=13.2,8.1,3.1 \mathrm{~Hz}, 1 \mathrm{H}, \mathbf{K}_{\mathbf{B}}\right), 2.68-2.78(\mathrm{~m}, 16 \mathrm{H}, \mathbf{B}), 3.02$ $(\mathrm{s}, 3 \mathrm{H}, \mathbf{N}), 4.65(\mathrm{dt}, J=7.4,3.7 \mathrm{~Hz}, 1 \mathrm{H}, \mathbf{J}), 6.41(\mathrm{~d}, J=7.4 \mathrm{~Hz}, 1 \mathrm{H}, \mathbf{I})$, $6.98(\mathrm{t}, J=1.4 \mathrm{~Hz}, 4 \mathrm{H}, \mathbf{E 1}), 7.16-7.18(\mathrm{~m}, 16 \mathrm{H}, \mathbf{A 2}), 7.31(\mathrm{t}, J=7.7$ $\mathrm{Hz}, 8 \mathrm{H}, \mathbf{A 1}), 7.42(\mathrm{~d}, J=1.4 \mathrm{~Hz}, 8 \mathrm{H}, \mathbf{E 3}), 7.44-7.46$ (m, 2H, G3), 7.48$7.51(\mathrm{~m}, 1 \mathrm{H}, \mathbf{G 4}), 7.68(\mathrm{~d}, J=1.4 \mathrm{~Hz}, 4 \mathrm{H}, \mathbf{F 3}), 7.79(\mathrm{t}, J=1.4 \mathrm{~Hz}, 2 \mathrm{H}, \mathbf{F 1}) ;{ }^{13} \mathbf{C}$ NMR $\left(125 \mathrm{MHz}, \mathrm{CDCl}_{3}\right): \delta 19.3$ (t, K), 24.3, 24.52, 24.54 (q, C and D), 30.5, 30.6 (d, B), 52.4 (q, N), 52.7 (d, J), 122.7 (d, A2), 126.2 (d, F1), 126.7 (d, E3), 127.2 (d, F3), 128.1 (d, A1), 129.4 (d, G4), 129.9 (d, G3), 130.5 (d, E1), 135.0 (s, G1), 139.2 (s, A4), 140.2 (s, G2), 140.6 (s, F2), 141.2 (s, E2), 141.7 (s, F4), 141.9 (s, E4), 146.9 (s, A3), 168.0 (s, H), 169.8 (s, M); ${ }^{77} \mathbf{S e}$ NMR (95 MHz, $\left.\mathrm{CDCl}_{3}\right): \delta-83.2 ; \mathbf{I R}(\mathrm{KBr}) ; 3419,3060,3033,2960,2926,2867,1749,1680,1578,1495,1460$, 1382, 1361, 1344, 1325, 1250, 1208, 1178, 1102, 1054, 873, 805, 754, $725 \mathrm{~cm}^{-1}$. HRMS (FD-TOF) $\mathrm{m} / \mathrm{z} 2024.1951$ $[\mathrm{M}]^{+}$(calcd for $\left.\mathrm{C}_{143} \mathrm{H}_{165} \mathrm{NO}_{3} \mathrm{Se}, 2024.1954\right)$. Anal. Calcd. for $\mathrm{C}_{143} \mathrm{H}_{165} \mathrm{NO}_{3} \mathrm{Se}: \mathrm{C}, 84.82 ; \mathrm{H}, 8.21 ; \mathrm{N}$, 0.69. Found: C, $85.03 ; \mathrm{H}, 7.95 ; \mathrm{N}, 0.75 \%$. 
Synthesis of $N$-Boc-Sec(CHPh $)$-OMe (S7).

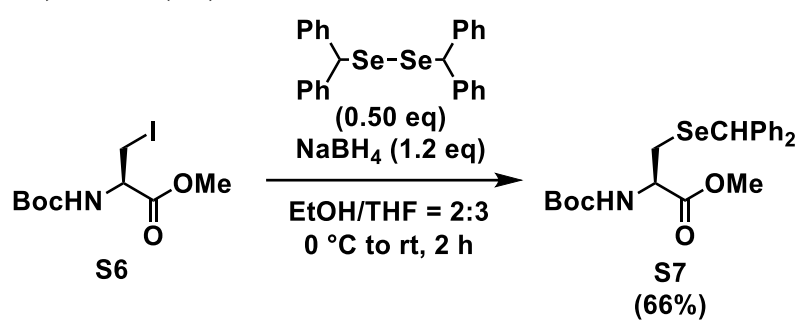

Scheme S5. Synthesis of $N$-Boc-Sec $\left(\mathrm{CHPh}_{2}\right)-\mathrm{OMe}(\mathbf{S 7})$.

Freshly prepared $N$-Boc-L-iodoalanine methyl ester $(\mathbf{S 6} ; 4.37 \mathrm{~g}, 13.3 \mathrm{mmol})$, which was prepared by the reported procedure, ${ }^{4}$ and bis(diphenylmethyl)diselenide $(3.27 \mathrm{~g}, 6.64 \mathrm{mmol})$, which was synthesized by the reported procedure, ${ }^{5}$ were placed in a $300 \mathrm{~mL}$ two-necked Schlenk flask. After evacuated and backfilled with argon, THF (36 mL), degassed EtOH $(24 \mathrm{~mL})$ and then $\mathrm{NaBH}_{4}(602 \mathrm{mg}, 15.9 \mathrm{mmol})$ were added in an ice bath. The reaction mixture was allowed to slowly warm to room temperature, after which it was stirred for an additional $2 \mathrm{~h}$ at the same temperature. After addition of water at $0{ }^{\circ} \mathrm{C}$, the two layer was separated and the aqueous layer was extracted with AcOEt $(3 \times 40 \mathrm{~mL})$. The combined organic layer was washed with brine, dried over $\mathrm{Na}_{2} \mathrm{SO}_{4}$, and filtered. The filtrate was concentrated in vacuo and the crude product was purified by flash column chromatography on silica gel (hexane/ $\mathrm{CHCl}_{3}=1: 6$ then 1:2) to give $\mathbf{S} 7$ as pale-yellow oil. The oil was crystalized in fridge and afforded as colorless crystals. Yield $3.93 \mathrm{~g}(8.77 \mathrm{mmol}, 66 \%)$.

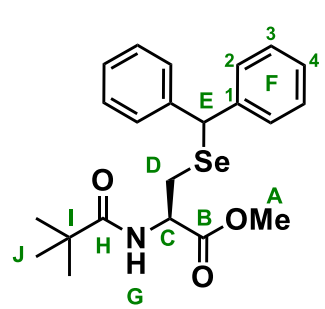

$\mathrm{N}$-Boc-Sec(CHPh $)$-OMe (S7) ${ }^{6}$ : colorless crystals; m.p. $79.2-79.9^{\circ} \mathrm{C} .{ }^{1} \mathbf{H} \mathbf{N M R}(500 \mathrm{MHz}$ $\left.\mathrm{CDCl}_{3}\right): \delta 1.45(\mathrm{~s}, 9 \mathrm{H}, \mathbf{J}), 2.75\left(\mathrm{dd}, J=12.9,6.0 \mathrm{~Hz}, 1 \mathrm{H}, \mathbf{D}_{\mathrm{A}}\right), 2.80(\mathrm{dd}, J=12.9,4.9 \mathrm{~Hz}$, 1H, $\mathbf{D}_{\mathbf{B}}$ ), 3.70 (s, 3H, A), 4.57 (br, 1H, C), 5.22 (br d, $\left.J=8.0 \mathrm{~Hz}, 1 \mathrm{H}, \mathbf{G}\right), 5.44$ (s, 1H, E), 7.19- 7.22 (m, 2H, F4), 7.27-7.31 (m, 4H, F3), 7.40-7.44 (m, 4H, F2); ${ }^{13} \mathbf{C ~ N M R ~ ( 1 2 5 ~ M H z ~}$ $\left.\mathrm{CDCl}_{3}\right): \delta 27.7$ (t, D), $28.4(\mathrm{q}, \mathbf{J}), 48.2(\mathrm{~d}, \mathbf{E}), 52.5$ (q, A), 53.3 (d, C), 80.1 (s, I), 127.3 (d, F4), 128.71, 128.78 (d, F2 and d, F3), 141.1 (s, F1), 155.1 (s, H), 171.7 (s, B); ${ }^{77}$ Se NMR (95 MHz, $\mathrm{CDCl}_{3}$ ): $\delta$ 309; IR (KBr); 3401, 2976, 2949, 2931, 1752, 1701, 1506, 1450, 1415, 1392, 1369, 1317, 1271, 1258, 1222, 1202, 1177, 1159, 1083, 1072, 1043, 1029, 1016, 973, 866, 819, 750, 706, 694, $622 \mathrm{~cm}^{-1}$, HRMS (FD-TOF) $\mathrm{m} / z$ 449.1114 [M] ${ }^{+}$(calcd for $\mathrm{C}_{22} \mathrm{H}_{27} \mathrm{NO}_{4} \mathrm{Se}, 449.1105$ ). Anal. Calcd. for $\mathrm{C}_{22} \mathrm{H}_{27} \mathrm{NO}_{4} \mathrm{Se}$ : C, 58.93; $\mathrm{H}$, 6.07; N, 3.12. Found: C, 58.86; H, 6.09; N, 3.09\%.

\section{Synthesis of $N$-Boc-Sec(CHPh $)$-OH (S8).}

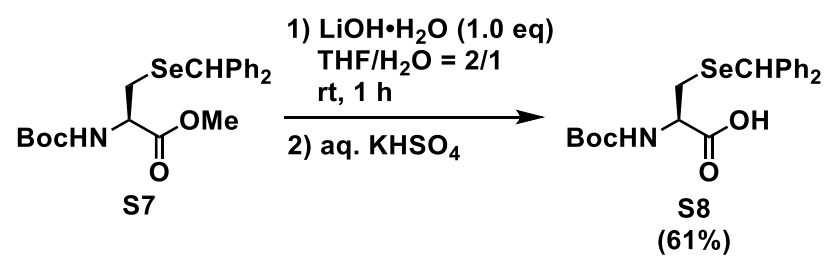

Scheme S6. Synthesis of $N$-Boc-Sec(CHPh $)-\mathrm{OH}(\mathbf{S 8})$.

Selenocysteine methyl ester $\mathbf{S} 7(1.53 \mathrm{~g}, 3.41 \mathrm{mmol})$ was placed in a $100 \mathrm{~mL}$ two-necked Schlenk flask. After evacuated and backfilled with argon, THF $(20 \mathrm{~mL})$, degassed water $(10 \mathrm{~mL})$ and then $\mathrm{LiOH} \cdot \mathrm{H}_{2} \mathrm{O}(143 \mathrm{mg}, 3.41$ mmol) were added. The resulting mixture was stirred at room temperature for $1 \mathrm{~h}$ before $10 \%$ aqueous $\mathrm{KHSO}_{4}$ was 


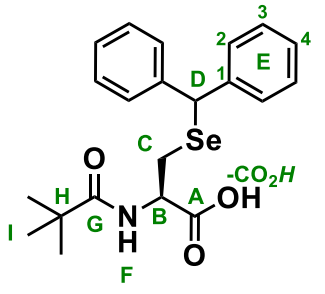

added. The two layers were rapidly separated and the aqueous layer was extracted with AcOEt $(3 \times 40 \mathrm{~mL})$. The combined organic layer was washed with brine, dried over $\mathrm{Na}_{2} \mathrm{SO}_{4}$, and filtered. The filtrate was concentrated in vacuo and the crude product was recrystallized from $\mathrm{Et}_{2} \mathrm{O}$-hexane ${ }^{6}$ to give $\mathbf{S 8}$ as colorless crystals. Yield $905 \mathrm{mg}$ (2.08 mmol, 61\%). The purity of this compound was crucial for next condensation reactions.

$N$-Boc-Sec(CHPh $)$ )-OH (S8) ${ }^{6}$ : colorless crystals; m.p. 91.3-92.9 ${ }^{\circ} \mathrm{C} .{ }^{1} \mathbf{H} \mathbf{~ N M R ~ ( 5 0 0 ~ M H z , ~} \mathrm{CDCl}_{3}$ ): $\delta 1.44$ (s, $9 \mathrm{H}$, I), 2.81 (br, 2H, C), 4.52 (br, 1H, B), 4.84 (br, $\left.1 \mathrm{H},-\mathbf{C O}_{2} \mathbf{H}\right), 5.20$ (br d, $\left.J=7.5 \mathrm{~Hz}, \mathbf{F}\right), 5.46$ (s, 1H, D), 7.18- 7.22 (m, 2H, E4), 7.25-7.30 (m, 4H, E3), 7.39-7.43 (m, 4H, E2); ${ }^{13} \mathbf{C ~ N M R ~ ( 1 2 5 ~ M H z , ~ C D C l ~} 3$ ): $\delta 27.3(\mathrm{t}, \mathbf{C}), 28.5(\mathrm{q}, \mathbf{I})$, 48.5 (d, D), 53.3 (d, B), 80.8 (s, H), 127.5 (d, E4), 128.8 (d, E2 and d, E3), 141.1 (s, E1), 155.7 (s, G), 175.2 (s, A); ${ }^{77}$ Se NMR (95 MHz, CDCl $): \delta 310$; IR (KBr); 3383, 3026, 3006, 2980, 2933, 1733, 1686, 1509, 1446, 1418 , 1393, 1364, 1339, 1279, 1214, 1168, 1074, 1048, 1024, 1003, $864 \mathrm{~cm}^{-1}$. HRMS (FD-TOF) $\mathrm{m} / z$ 435.0965 [M] ${ }^{+}$(cald for $\mathrm{C}_{21} \mathrm{H}_{25} \mathrm{NO}_{4} \mathrm{Se}, 435.0948$ ).

\section{Synthesis of $N$-Boc-Sec(CHPh 2$)$-Gly-Gly-OMe (S10).}

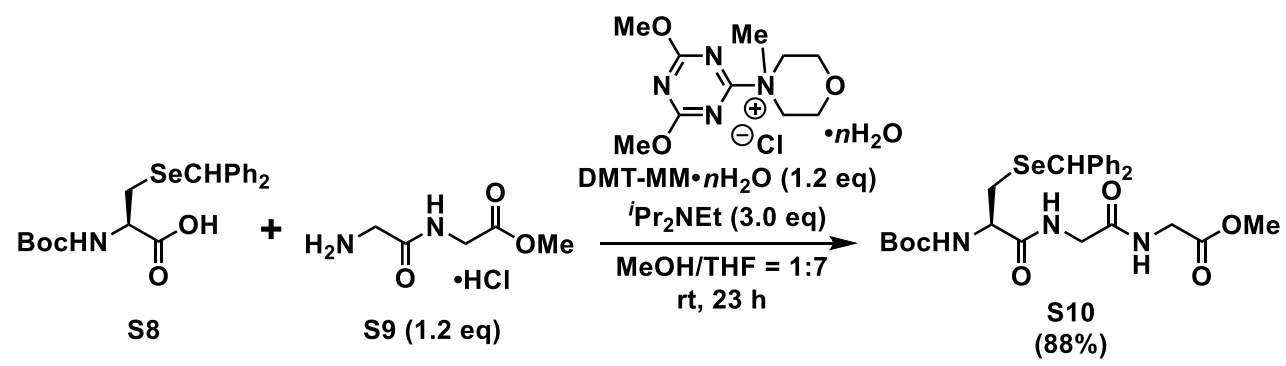

Scheme S7. Synthesis of $N$-Boc-Sec( $\left.\mathrm{CHPh}_{2}\right)$-Gly-Gly-OMe (S10).

Selenocysteine S8 (760 mg, $1.76 \mathrm{mmol}$ ) and glycylglycine methyl ester hydrochloride (S9; $584 \mathrm{mg}, 2.11 \mathrm{mmol}$ ), which was prepared by the reported procedure, ${ }^{7}$ were placed in a $50 \mathrm{~mL}$ Schlenk tube. After evacuated and backfilled with argon, THF $(12 \mathrm{~mL}), \mathrm{MeOH}(1.6 \mathrm{~mL}),{ }^{i} \mathrm{Pr}_{2} \mathrm{NEt}(0.90 \mathrm{~mL}, 5.3 \mathrm{mmol})$ and then DMT-MM $n$-hydrate (584 mg, $2.11 \mathrm{mmol}$ ) were added. The resulting mixture was stirred at room temperature for $23 \mathrm{~h}$. The two layers were separated after addition of water and the aqueous layer was extracted with $\mathrm{CHCl}_{3}(3 \times 15 \mathrm{~mL})$. The combined organic layer was washed with brine, dried over $\mathrm{Na}_{2} \mathrm{SO}_{4}$, and filtered. The filtrate was concentrated in vacuo and the crude product was purified by flash column chromatography on silica gel $\left(\mathrm{CH}_{2} \mathrm{Cl}_{2} / \mathrm{MeOH}=50: 1\right)$ to give $\mathbf{S 1 0}$ as colorless crystals. Yield $867 \mathrm{mg}(1.54 \mathrm{mmol}, 88 \%)$.

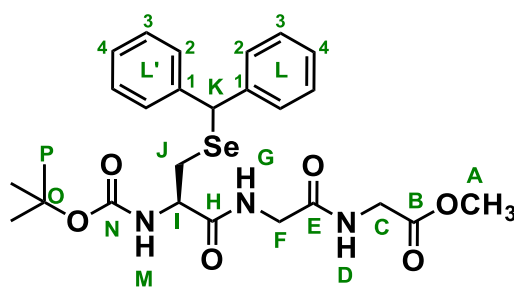

$N$-Boc-Sec(CHPh $)$-Gly-Gly-OMe (S10): colorless crystals; m.p. 45.2$46.3{ }^{\circ} \mathrm{C} .{ }^{1} \mathbf{H}$ NMR $\left(500 \mathrm{MHz}, \mathrm{CDCl}_{3}\right): \delta 1.44$ (s, 9H, P), 2.75 (dd, $J=12.9$, $\left.6.0 \mathrm{~Hz}, 1 \mathrm{H}, \mathbf{J}_{\mathrm{A}}\right), 2.83\left(\mathrm{dd}, J=13.2,6.6 \mathrm{~Hz}, 1 \mathrm{H}, \mathbf{J}_{\mathbf{B}}\right), 3.71(\mathrm{~s}, 3 \mathrm{H}, \mathbf{A}), 3.94(\mathrm{~d}$, $J=5.7 \mathrm{~Hz}, 2 \mathrm{H}, \mathbf{F}), 3.96(\mathrm{~d}, J=6.0 \mathrm{~Hz}, 2 \mathrm{H}, \mathbf{C}), 4.17(\mathrm{q}, J=6.6 \mathrm{~Hz}, 1 \mathrm{H}, \mathbf{I})$, 5.13 (br, 1H, M), 5.44 (s, 1H, K), 6.73 (br, 1H, D), 6.89 (br, 1H, G), 7.217.24 (m, 2H, L4 and L'4), 7.28-7.32 (m, 4H, L3 and L'3), 7.43-7.45 (m, 4H, L2 and L'2); ${ }^{13} \mathbf{C}$ NMR (125 MHz, $\mathrm{CDCl}_{3}$ ): $\delta 27.4$ (t, J), 28.3 (q, P), 41.0 (t, F), 43.0 (t, C), 48.3 (d, K), 52.3 (q, A), 54.4 (d, I), 80.4 (s, O), 127.3 (d, L4 and L'4), 128.6 (d, L2 and L'2, overlapped, d, L3 and L'3), 141.2 (s, L1), 155.7 (s, N), 169.3 (s, B), 170.2 (s, H), 171.7 (s, E); ${ }^{77}$ Se NMR (95 MHz, $\left.\mathrm{CDCl}_{3}\right): \delta 312$; IR (KBr); 3317, 3083, 3060, 3025, 3003, 2977, 2952, 2933, 
1749, 1683, 1670, 1523, 1508, 1494, 1449, 1438, 1408, 1392, 1367, 1318, 1247, 1213, 1165, 1077, 1045, 1030, 1021, 984, 862, 751, $700 \mathrm{~cm}^{-1}$, HRMS (FD-TOF) $\mathrm{m} / z 563.1559[\mathrm{M}]^{+}$(calcd for $\mathrm{C}_{26} \mathrm{H}_{33} \mathrm{~N}_{3} \mathrm{O}_{6} \mathrm{Se}, 563.1534$ ).

Synthesis of H-Sec(CHPh $)$-Gly-Gly-OMe hydrochloride (S11).

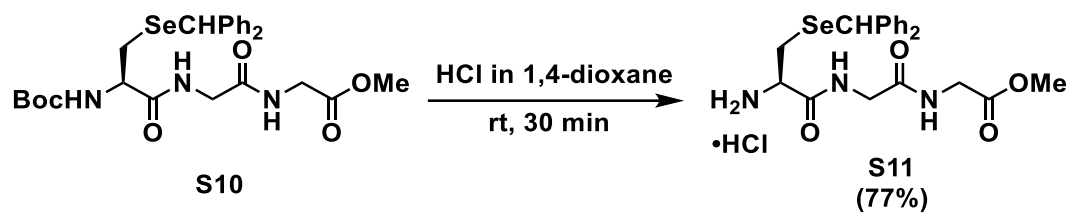

Scheme S8. Synthesis of H-Sec( $\left.\mathrm{CHPh}_{2}\right)$-Gly-Gly-OMe hydrochloride (S11).

A $N$-Boc-Sec(CHPh $)$-Gly-Gly-OMe $(\mathbf{S 1 0} ; 338 \mathrm{mg}, 0.586 \mathrm{mmol})$ was placed in a $25 \mathrm{~mL}$ Schlenk tube. After evacuated and backfilled with argon, a $4 \mathrm{M}$ 1,4-dioxane solution of $\mathrm{HCl}(4.7 \mathrm{~mL}, 19 \mathrm{mmol})$ was added. The resulting solution was stirred at room temperature for $30 \mathrm{~min}$. The solution was concentrated in vacuo and the crude product was dried under vacuum at $55^{\circ} \mathrm{C}$ to give $\mathbf{S 1 1}$ as off-white crystals. Yield $225 \mathrm{mg}(0.451 \mathrm{mmol}, 77 \%)$. This compound was sufficiently dried and used for the next reaction because of its hygroscopicity.

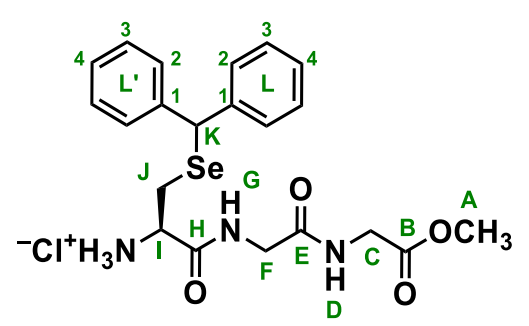

H-Sec(CHPh $)$-Gly-Gly-OMe hydrochloride (S11): off-white crystals; m.p. 99.9-102.8 ${ }^{\circ} \mathrm{C} .{ }^{1} \mathbf{H}$ NMR $\left(500 \mathrm{MHz}, \mathrm{CD}_{3} \mathrm{OD}\right): \delta 2.82(\mathrm{dd}, J=13.3,6.6 \mathrm{~Hz}$, $\left.1 \mathrm{H}, \mathbf{J}_{\mathbf{A}}\right), 2.89\left(\mathrm{dd}, J=13.3,6.6 \mathrm{~Hz}, 1 \mathrm{H}, \mathbf{J}_{\mathbf{B}}\right), 3.70(\mathrm{~s}, 3 \mathrm{H}, \mathbf{A}), 3.89\left(\mathrm{ABq}, J_{\mathrm{AB}}\right.$ $\left.=16.4 \mathrm{~Hz}, 2 \mathrm{H}, \mathbf{F}_{\mathbf{A}}\right), 4.12\left(\mathrm{ABq}, J_{\mathrm{AB}}=17.0 \mathrm{~Hz}, 2 \mathrm{H}, \mathbf{F}_{\mathbf{B}}\right), 3.96(\mathrm{~s}, 2 \mathrm{H}, \mathbf{C}), 4.06$ (t, $J=7.0 \mathrm{~Hz}, 2 \mathrm{H}, \mathbf{I}), 5.65$ (s, 1H, K), 7.21-7.25 (m, 2H, L4 and L'4), 7.307.35 (m, 4H, L2 and L'2), 7.48-7.51 (m, 4H, L3 and L'3); ${ }^{\mathbf{1 3}} \mathbf{C}$ NMR (125 MHz, CD 3 OD): $\delta 25.7$ (t, J), 41.7 (t, C), 43.2 (t, F), 49.4 (d, K), 52.6 (q, A), 54.1 (d, I), 128.45 (d, L4 or L'4), 128.49 (d, L4 or L'4), 129.71 (d, L2 or L'2), 129.78 (d, L2 or L'2, overlapped, L3 and L'3), 142.2 (s, L1 or L'1), 142.3 (s, L1 or L'1), 169.5 (s, H), 171.3 (s, E), 171.5 (s, B); ${ }^{77}$ Se NMR (95 MHz, CD 3 OD): $\delta 295$; IR (KBr); 3347 , 3212, 3067, 3024, 2932, 2853, 2830, 2499, 2374, 2195, 2069, 1747, 1670, 1655, 1557, 1506, 1493, 1474, 1447, 1338, 1289, 1251, 1216, 1184, 1020, 1002, 978, 774, 748, 700, $579 \mathrm{~cm}^{-1}$. HRMS (FAB) $\mathrm{m} / \mathrm{z} 464.1092$ [M-Cl] (calcd for $\mathrm{C}_{21} \mathrm{H}_{26} \mathrm{~N}_{3} \mathrm{O}_{4} \mathrm{Se}^{+}$, M-Cl, 464.1090).

\section{Synthesis of $N$-Bpsc-Se-diphenylmethyl tripeptide 4.}

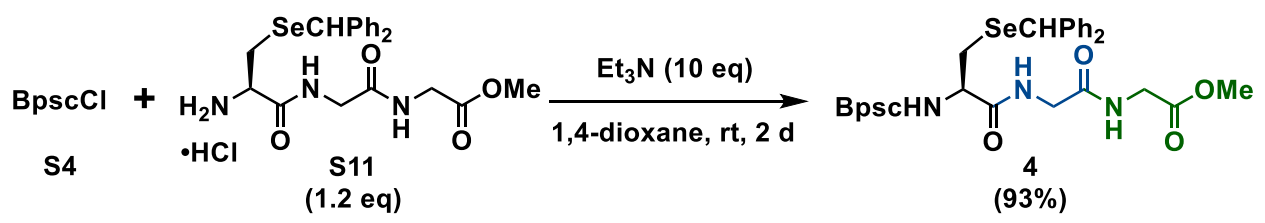

Scheme S9. Synthesis of $N$-Bpsc-Se-diphenylmethyl tripeptide 4.

Selenopeptide S11 (115 mg, $0.230 \mathrm{mmol}$ ) was placed in a $25 \mathrm{~mL}$ Schlenk tube. After evacuated for $1 \mathrm{~h}$ and backfilled with argon, 1,4-dioxane (7.0 mL), $\mathrm{Bpscl}(\mathbf{S 4} ; 360 \mathrm{mg}, 0.192 \mathrm{mmol})$ and then $\mathrm{Et}_{3} \mathrm{~N}(0.27 \mathrm{~mL}, 1.9 \mathrm{mmol})$ were added. The resulting suspension was stirred at room temperature for $49 \mathrm{~h}$ before $10 \%$ aqueous $\mathrm{NH}_{4} \mathrm{Cl}$ was added. The two layers were separated and the aqueous layer was extracted with $\mathrm{CH}_{2} \mathrm{Cl}_{2}(3 \times 8 \mathrm{~mL})$. The combined organic layer was washed with brine, dried over $\mathrm{Na}_{2} \mathrm{SO}_{4}$, and filtered. The filtrate was concentrated in vacuo and 
the crude product was purified by flash column chromatography on silica gel (hexane/ $\mathrm{CH}_{2} \mathrm{Cl}_{2}=1: 4$ then 1:6) then triturated with $\mathrm{CH}_{2} \mathrm{Cl}_{2}$-EtOH to give the 4 as colorless crystals. Yield $413 \mathrm{mg}(0.179 \mathrm{mmol}, 93 \%)$.

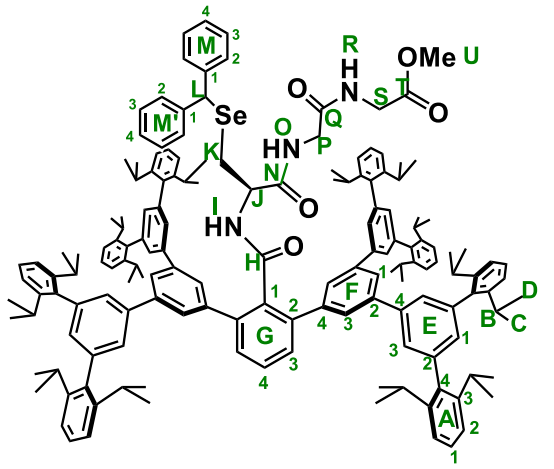

4: colorless crystals; m.p. $195.3-196.7^{\circ} \mathrm{C} .{ }^{1} \mathbf{H}$ NMR $\left(500 \mathrm{MHz}, \mathrm{CDCl}_{3}\right): \delta$ 1.03-1.05 (m, 48H, C and D), 1.08-1.11 (m, 48H, C and D), 2.08 (dd, $J=$ $\left.12.9,7.4 \mathrm{~Hz}, 1 \mathrm{H}, \mathbf{K}_{\mathbf{A}}\right), 2.27\left(\mathrm{dd}, J=12.9,5.7 \mathrm{~Hz}, 1 \mathrm{H}, \mathbf{K}_{\mathbf{B}}\right), 2.72-2.79(\mathrm{~m}$, 16H, B), $3.01\left(\mathrm{dd}, J=16.6,5.2 \mathrm{~Hz}, 1 \mathrm{H}, \mathbf{P}_{\mathbf{A}}\right), 3.42-3.49\left(\mathrm{~m}, 2 \mathrm{H}, \mathbf{P}_{\mathbf{B}}\right.$ and $\left.\mathbf{S}_{\mathbf{A}}\right)$, $3.53(\mathrm{~s}, 3 \mathrm{H}, \mathbf{U}), 3.51-3.56\left(\mathrm{~m}, 1 \mathrm{H}, \mathbf{S}_{\mathbf{B}}\right), 3.98-4.02(\mathrm{~m}, 1 \mathrm{H}, \mathbf{J}), 4.99(\mathrm{~s}, 1 \mathrm{H}, \mathbf{L})$, $6.01(\mathrm{~d}, J=7.2 \mathrm{~Hz}, 1 \mathrm{H}, \mathbf{I}), 6.21(\mathrm{t}, J=6.0 \mathrm{~Hz}, 1 \mathrm{H}, \mathbf{O}), 6.45(\mathrm{t}, J=5.7 \mathrm{~Hz}$, $1 \mathrm{H}, \mathbf{R}), 6.83(\mathrm{t}, J=7.7 \mathrm{~Hz}, 2 \mathrm{H}, \mathbf{M} 2$ or M'2), 6.87 (t, $J=7.7 \mathrm{~Hz}, 2 \mathrm{H}, \mathbf{M} 2$ or M'2), 6.93-6.97 (m, 2H, M4 and M'4), 7.01 (br, 4H, E1), 7.01-7.04 (m, 4H, M3 and M'3), 7.18-7.20 (m, 16H, A2), $7.33(\mathrm{t}, J=7.7 \mathrm{~Hz}, 8 \mathrm{H}, \mathbf{A 1}), 7.41(\mathrm{~d}, J=1.4 \mathrm{~Hz}, 8 \mathrm{H}, \mathbf{E 3}), 7.41-7.43(\mathrm{~m}, 2 \mathrm{H}$, G3), 7.48-7.51 (m, 1H, G4), $7.64(\mathrm{~d}, J=1.4 \mathrm{~Hz}, 4 \mathrm{H}, \mathbf{F 3}), 7.77$ (br, 2H, F1); ${ }^{13} \mathbf{C}$ NMR $\left(125 \mathrm{MHz}, \mathrm{CDCl}_{3}\right): \delta 24.3$, 24.53, 24.56 (q, C and D), 25.5 (t, K), 30.6 (d, B), 40.7 (t, S), 42.6 (t, P), 48.2 (d, L), 52.1 (q, U), 53.1 (d, J), 122.7 (d, A2), 126.0 (d, F1), 126.6 (d, E3), 127.14, 127.17 (d, M4 and M'4), 127.3 (d, F3), 128.1 (d, A1), 128.4 (d, M2 and M'2), 128.5 (d, M3 and M'3), 129.5 (d, G4), 130.0 (d, G3), 130.6 (d, E1), 134.5 (s, G1), 139.1 (s, A4), 140.0 (s, G2), 140.4 (s, F2), 141.1 (s, M1, and M'1, overlapped, s, F4), 141.3 (s, E2), 141.8 (s, E4), 146.90, 146.93 (s, A3), 168.3 (s, Q), 169.1 (s, H), 169.7 (s, T), 169.8 (s, N); ${ }^{77} \mathbf{S e}$ NMR (95 MHz, CDCl $)$ ): $\delta 315$. IR (KBr); 3402, 3371, 3060, 3030, 2961, 2926, 2867, 1750, 1691, 1593, 1578, 1507, 1493, 1462, 1382, 1361, 1345, 1325, 1249, $1205,1179,1054,873,805,754,725,699 \mathrm{~cm}^{-1}$; HRMS (FD-TOF) $\mathrm{m} / 22304.3273[\mathrm{M}]^{+}\left(\right.$calcd for $\mathrm{C}_{160} \mathrm{H}_{181} \mathrm{~N}_{3} \mathrm{O}_{5} \mathrm{Se}$, 2304.3166).

\section{Synthesis of selenenyl iodide 5 .}

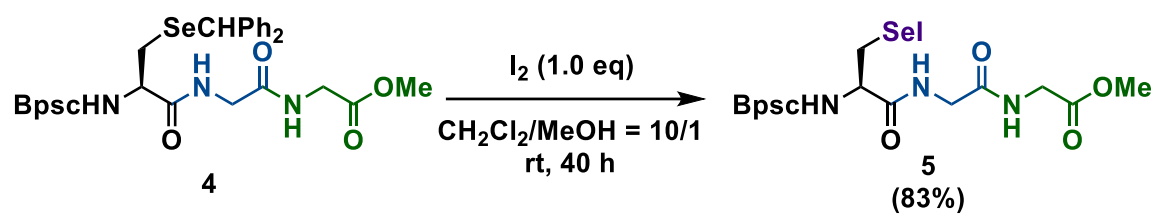

Scheme S10. Synthesis of selenenyl iodide 5 .

Selenide 4 (47.5 mg, $20.6 \mu \mathrm{mol})$ was placed in a J-young $10 \mathrm{~mL}$ Schlenk tube. After evacuated and backfilled with argon, $\mathrm{CH}_{2} \mathrm{Cl}_{2}(1.0 \mathrm{~mL}), \mathrm{MeOH}(0.1 \mathrm{~mL})$ and then $\mathrm{I}_{2}(5.8 \mathrm{mg}, 21 \mu \mathrm{mol})$ were added. The resulting solution was stirred at room temperature for $40 \mathrm{~h}$ after the tube was carefully sealed. After addition of $\mathrm{CH}_{3} \mathrm{CN}, 90 \%$ of the solvent was removed in vacuo. The obtained suspension was cooled and filtered, and the resulting solid was washed with cold $\mathrm{CH}_{3} \mathrm{CN}$ to give 5 as reddish-purple crystals. Yield $38.5 \mathrm{mg}(17.0 \mu \mathrm{mol}, 83 \%)$.

Selenenyl iodide 5: reddish-purple crystals; m.p. $243.1-244.2{ }^{\circ} \mathrm{C}$ (dec.). ${ }^{1} \mathbf{H}$ NMR (500 MHz, $\left.\mathrm{CDCl}_{3}\right) \delta 1.02-1.04$ (m, 48H, C and D), 1.09-1.12 (m, 48H, C and D), $2.57\left(\mathrm{dd}, J=13.3,5.6 \mathrm{~Hz}, 1 \mathrm{H}, \mathbf{K}_{\mathbf{A}}\right), 2.70-2.78(\mathrm{~m}, 16 \mathrm{H}, \mathbf{B}), 2.89$ (dd, $\left.J=13.3,4.7 \mathrm{~Hz}, 1 \mathrm{H}, \mathbf{K}_{\mathbf{B}}\right), 3.05\left(\mathrm{dd}, J=16.6,5.5 \mathrm{~Hz}, 1 \mathrm{H}, \mathbf{N}_{\mathbf{A}}\right), 3.38\left(\mathrm{dd}, J=16.6,6.3 \mathrm{~Hz}, 1 \mathrm{H}, \mathbf{N}_{\mathbf{B}}\right), 3.59$ (s, $3 \mathrm{H}$, S), 3.65-3.67 (m, 2H, $\mathbf{Q}_{\mathbf{A}}$ and $\left.\mathbf{Q}_{\mathbf{B}}\right), 4.17-4.20(\mathrm{~m}, 1 \mathrm{H}, \mathbf{J}), 6.18(\mathrm{t}, J=5.3 \mathrm{~Hz}, 1 \mathrm{H}, \mathbf{P}), 6.39(\mathrm{t}, J=5.9 \mathrm{~Hz}, 1 \mathrm{H}, \mathbf{M})$, $6.50(\mathrm{~d}, J=6.6 \mathrm{~Hz}, 1 \mathrm{H}, \mathbf{I}), 7.00$ (br, 4H, E1), 7.17 (d, $J=7.8 \mathrm{~Hz}, 16 \mathrm{H}, \mathbf{A 2}), 7.31$ (t, $J=7.8 \mathrm{~Hz}, 8 \mathrm{H}, \mathbf{A 1}), 7.43-7.45$ (m, 2H, G3), 7.44 (d, $J=1.4 \mathrm{~Hz}, 8 \mathrm{H}, \mathbf{E 3 ) , ~ 7 . 5 0 - 7 . 5 3 ~ ( m , ~ 1 H , ~ G 4 ) , ~} 7.66$ (d, $J=1.7 \mathrm{~Hz}, 4 \mathrm{H}, \mathbf{F 3}), 7.85$ (br, 2H, F1); 


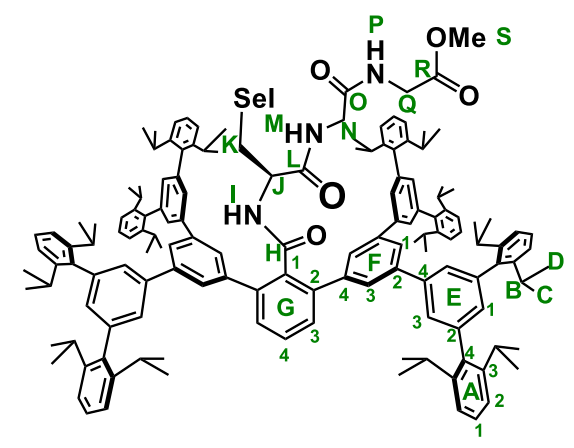
$1362,1346,1325,1313,1250,1211,1179,1160,1101,1055,1038,1024,873,805,762,754,724 \mathrm{~cm}^{-1}$; UV-vis $\left(\mathrm{CHCl}_{3}, 298 \mathrm{~K}\right) \lambda_{\max } 482 \mathrm{~nm}(\varepsilon=86)$, HRMS (FD-TOF) $m / z 2264.1372[\mathrm{M}]^{+}$(calcd for $\mathrm{C}_{147} \mathrm{H}_{170} \mathrm{IN}_{3} \mathrm{O}_{5} \mathrm{Se}$, 2264.1350).

\section{Synthesis of selenenyl sulfide $7 \mathrm{~b}$.}
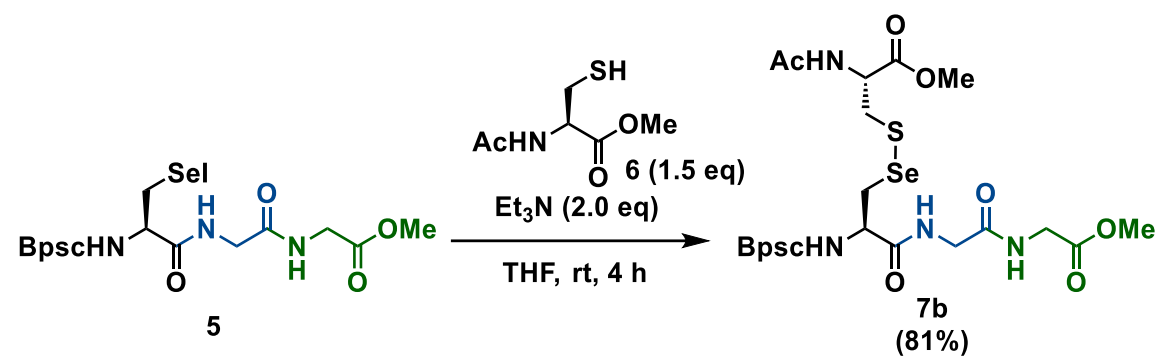

Scheme S11. Synthesis of selenenyl sulfide $7 \mathbf{b}$.

Selenenyl iodide 5 (307 mg, $0.135 \mathrm{mmol})$ was placed in a $25 \mathrm{~mL}$ Schlenk tube. After evacuated and backfilled with argon, THF $(7.0 \mathrm{~mL}), \mathrm{Et}_{3} \mathrm{~N}(38 \mu \mathrm{L}, 0.27 \mathrm{mmol})$ and then $N$-acetyl cysteine methyl ester $(\mathbf{6} ; 36 \mathrm{mg}, 0.20 \mathrm{mmol})$ were added. The resulting solution was stirred at room temperature for $4 \mathrm{~h}$ before $10 \%$ aqueous $\mathrm{NH}_{4} \mathrm{Cl} \mathrm{was}_{\text {added. }}$ The two layers were separated and the aqueous layer was extracted with $\mathrm{CH}_{2} \mathrm{Cl}_{2}(3 \times 8 \mathrm{~mL})$. The combined organic layer was washed with brine, dried over $\mathrm{Na}_{2} \mathrm{SO}_{4}$, and filtered. The filtrate was concentrated in vacuo and the crude product was purified by flash column chromatography on silica gel $\left(\mathrm{CH}_{2} \mathrm{Cl}_{2} / \mathrm{MeOH}=50: 1\right)$ to give $7 \mathbf{b}$ as colorless crystals. Yield $253 \mathrm{mg}(0.109 \mathrm{mmol}, 81 \%)$.

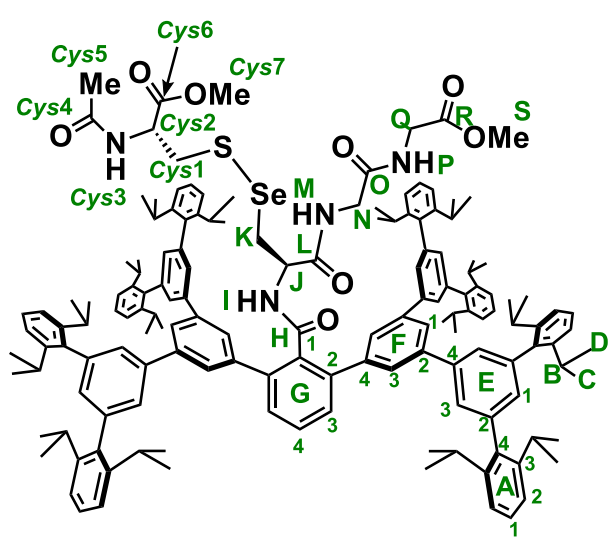

Selenenyl sulfide 7b: colorless crystals; m.p. 193.2-194.0 ${ }^{\circ} \mathrm{C} .{ }^{1} \mathbf{H}$ NMR $\left(500 \mathrm{MHz} \mathrm{CDCl}_{3}\right): \delta 1.02-1.04(\mathrm{~m}, 48 \mathrm{H}, \mathbf{C}$ and $\mathbf{D}), 1.08-1.12(\mathrm{~m}, 48 \mathrm{H}$, C and D), 1.61 (s, 3H, Cys5), $2.30\left(\mathrm{dd}, J=13.0,5.0 \mathrm{~Hz}, 1 \mathrm{H}, \mathbf{K}_{\mathrm{A}}\right), 2.65$ $\left(\mathrm{dd}, J=13.9,5.3 \mathrm{~Hz}, 1 \mathrm{H}, \boldsymbol{C y s} \mathbf{1}_{\mathrm{A}}\right), 2.69-2.79(\mathrm{~m}, 16 \mathrm{H}, \mathbf{B}), 2.87(\mathrm{dd}, J=$ 14.0, 5.4 Hz, 1H, Cys1 $\mathbf{1}_{\mathbf{B}}$ ), 3.01 (dd, J=12.6, 4.6 Hz, 1H, KB ), 3.04 (dd, $\left.J=15.3,5.5 \mathrm{~Hz}, 1 \mathrm{H}, \mathbf{N}_{\mathbf{A}}\right), 3.28\left(\mathrm{dd}, J=16.6,6.0 \mathrm{~Hz}, 1 \mathrm{H}, \mathbf{N}_{\mathbf{B}}\right), 3.53-$ $3.57\left(\mathrm{~m}, 1 \mathrm{H}, \mathbf{Q}_{\mathrm{A}}\right), 3.57$ (s, 3H, Cys7), 3.61 (s, 3H, S), $3.66(\mathrm{dd}, J=18.0$, $\left.5.5 \mathrm{~Hz}, 1 \mathrm{H}, \mathbf{Q}_{\mathbf{B}}\right), 4.35$ (dt, $J=7.2,5.4 \mathrm{~Hz}, 1 \mathrm{H}$, Cys2), 4.56 (dt, $J=8.0$, $4.7 \mathrm{~Hz}, 1 \mathrm{H}, \mathbf{J}), 6.21(\mathrm{t}, J=5.5 \mathrm{~Hz}, 1 \mathrm{H}, \mathbf{P}), 6.30(\mathrm{t}, J=5.7 \mathrm{~Hz}, 1 \mathrm{H}, \mathbf{M})$, 6.72 (d, J= 7.2 Hz, 1H, Cys3), 6.99 (br, 4H, E1), 7.02 (d, J=8.0 Hz, 1H, I), 7.16-7.18 (m, 16H, A2), 7.32 (t, J= 7.7 Hz, 8H, A1), 7.43 (br d, J = 1.2 Hz, 8H, E3), 7.43-7.45 (m, 2H, G3), 7.49-7.52 (m, 1H, G4), 7.69 (br, 4H, F3), 
7.82 (br, 2H, F1); ${ }^{13} \mathbf{C}$ NMR (125 MHz, $\mathrm{CDCl}_{3}$ ): $\delta 22.5$ (q, Cys5), 24.33, 24.36, 24.48 (q, C and D), 30.60, 30.62 (d, B), 33.1 (t, K), 39.7 (t, Cys1), 40.9 (t, Q), 42.4 (t, N), 52.2 (d, J, overlapped, q, S), 52.62 (d, Cys2), 52.66 (q, Cys7), 122.7 (d, A2), 126.1 (d, F1), 126.5 (d, E3), 127.2 (d, F3), 128.1 (d, A1), 129.5 (d, G4), 130.0 (d, G3), 130.6 (d, E1), 134.7 (s, G1), 139.1 (s, A4), 139.9 (s, G2), 140.2 (s, F2), 141.3 (s, E2), 141.4 (s, F4), 141.9 (s, E4), 146.9 (s, A3), 168.2 (s, O), 169.0 (s, H), 169.6 (s, L), 169.8 (s, R), 170.6 (s, Cys4), 171.0 (s, Cys6); ${ }^{77}$ Se NMR (95 MHz, $\left.\mathrm{CDCl}_{3}\right): \delta 382$; IR (KBr); 3403, 3060, 3032, 2961, 2926, 2867, 1748, 1685, 1577, 1559, 1508, 1463, 1438, 1418, 1397, 1382, 1362, 1345, 1325, 1310, 1250, 1209, 1179, 1055, 1038, 874, 805, 754, $725 \mathrm{~cm}^{-1}$; HRMS (FD-TOF) $\mathrm{m} / z, 2314.2730[\mathrm{M}]^{+}$(cald for $\mathrm{C}_{153} \mathrm{H}_{180} \mathrm{~N}_{4} \mathrm{O}_{8} \mathrm{SSe}, 2313.2680$ ).

\section{Synthesis of selenol $3 b$.}

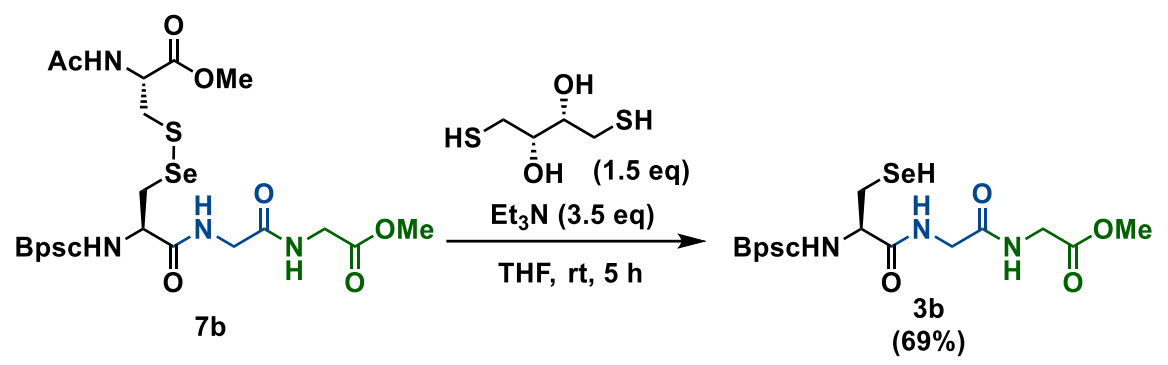

Scheme S12. Synthesis of selenol $\mathbf{3 b}$.

Selenenyl sulfide $7 \mathbf{b}(109 \mathrm{mg}, 46.9 \mu \mathrm{mol})$ was placed in a $25 \mathrm{~mL}$ Schlenk tube. After evacuated and backfilled with argon, THF (3.0 mL), Et $3 \mathrm{~N}(23 \mu \mathrm{L}, 0.16 \mathrm{mmol})$ and then D, L-dithiothreitol (11 mg, $70 \mu \mathrm{mol})$ were added. The resulting solution was stirred at room temperature for $5 \mathrm{~h}$. The following workup were performed under argon atmosphere. After diluted with $\mathrm{Et}_{2} \mathrm{O}$, degassed brine was added to the solution. The two layers were separated and the aqueous layer was extracted with $\mathrm{Et}_{2} \mathrm{O}(3 \times 4 \mathrm{~mL})$ using Schlenk tubes. The combined organic layer was dried over $\mathrm{Na}_{2} \mathrm{SO}_{4}$ and filtered using a glass filter. The filtrate was concentrated in vacuo and the crude product was immediately purified by column chromatography on silica gel (hexane/ $\mathrm{CH}_{2} \mathrm{Cl}_{2}=8: 1$ ) to give $\mathbf{3 b}$ as colorless crystals. Yield $68.9 \mathrm{mg}(32.2 \mu \mathrm{mol}, 69 \%)$.

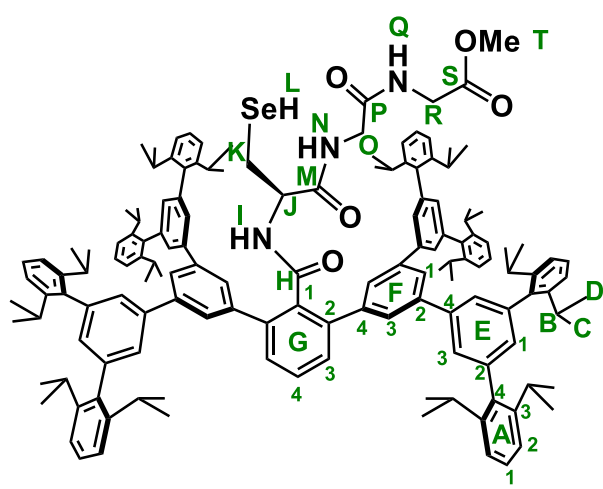

Selenol 3b: colorless crystals; m.p. 234.6-235.8 ${ }^{\circ} \mathrm{C} .{ }^{1} \mathbf{H}$ NMR $(500$ $\left.\mathrm{MHz}, \mathrm{CDCl}_{3}\right): \delta-1.07(\mathrm{dd}, J=8.9,7.2 \mathrm{~Hz}, \mathbf{L}), 1.03-1.05(\mathrm{~m}, 48 \mathrm{H}, \mathbf{C}$ and $\mathbf{D}), 1.09-1.13(\mathrm{~m}, 48 \mathrm{H}, \mathbf{C}$ and $\mathbf{D}), 1.96-2.01\left(\mathrm{~m}, 1 \mathrm{H}, \mathbf{K}_{\mathbf{A}}\right), 2.59(\mathrm{ddd}$, $\left.J=16.9,6.9,3.7 \mathrm{~Hz}, 1 \mathrm{H}, \mathbf{K}_{\mathbf{B}}\right), 2.67-2.79(\mathrm{~m}, 16 \mathrm{H}, \mathbf{B}), 3.01(\mathrm{dd}, J=$ $\left.16.6,4.9 \mathrm{~Hz}, 1 \mathrm{H}, \mathbf{O}_{\mathbf{A}}\right), 3.30\left(\mathrm{dd}, J=16.6,6.6 \mathrm{~Hz}, 1 \mathrm{H}, \mathbf{O}_{\mathbf{B}}\right), 3.60-3.65$ $\left(\mathrm{m}, 1 \mathrm{H}, \mathbf{R}_{\mathbf{A}}\right), 3.62(\mathrm{~s}, 3 \mathrm{H}, \mathbf{T}), 3.76\left(\mathrm{dd}, J=18.3,5.4 \mathrm{~Hz}, 1 \mathrm{H}, \mathbf{R}_{\mathbf{B}}\right), 4.34-$ $4.37(\mathrm{~m}, 1 \mathrm{H}, \mathbf{J}), 5.93(\mathrm{t}, J=5.7 \mathrm{~Hz}, 1 \mathrm{H}, \mathbf{N}), 6.16(\mathrm{t}, J=5.3 \mathrm{~Hz}, 1 \mathrm{H}, \mathbf{Q})$, $6.58(\mathrm{~d}, J=7.2 \mathrm{~Hz}, 1 \mathrm{H}, \mathbf{I}), 7.00(\mathrm{t}, J=1.5 \mathrm{~Hz}, 4 \mathrm{H}, \mathbf{E 1}), 7.17-7.22(\mathrm{~m}$, 16H, A2), 7.33 (t, $J=7.8 \mathrm{~Hz}, 8 \mathrm{H}, \mathbf{A 1}), 7.42$ (d, $J=1.5 \mathrm{~Hz}, 8 \mathrm{H}, \mathbf{E 3 ) , ~ 7 . 4 5 - 7 . 4 8 ~ ( m , ~ 2 H , ~ G 3 ) , ~ 7 . 5 1 - 7 . 5 4 ~ ( m , ~ 1 H , ~ G 4 ) , ~}$ $7.68(\mathrm{~d}, J=1.7 \mathrm{~Hz}, 4 \mathrm{H}, \mathbf{F 3}), 7.82(\mathrm{br}, 2 \mathrm{H}, \mathbf{F 1}) ;{ }^{13} \mathbf{C} \mathbf{~ N M R}\left(125 \mathrm{MHz}, \mathrm{CDCl}_{3}\right)$ : $\delta 18.8(\mathrm{t}, \mathbf{K}), 24.1,24.34,24.38,24.5$ (q, C and D), 30.6 (d, B), 40.9 (t, R), 42.5 (t, O), 52.4 (q, T), 53.3 (d, J), 122.7 (d, A2), 126.2 (d, F1), 126.6 (d, E3), 127.2 (d, F3), 128.1 (d, A1), 129.6 (d, G4), 129.9 (d, G3), 130.6 (d, E1), 134.8 (s, G1), 139.1 (s, A4), 139.9 (s, G2), 140.3 (s, E4), 141.3 (s, E2), 141.5 (s, F4), 142.0 (s, F2), 146.9 (s, A3), 168.2 (s, P), 168.9 (s, H), 169.3 (s, M), 
169.9 (s, S); ${ }^{77} \mathrm{Se} \mathbf{N M R}\left(95 \mathrm{MHz}, \mathrm{CDCl}_{3}\right.$ ): $\delta$-79.0; IR (KBr); 3395, 3060, 2961, 2926, 2867, 1750, 1694, 1579, 1509, 1462, 1382, 1362, 1325, 1250, 1208, 1038, 874, 828, 805, $754 \mathrm{~cm}^{-1}$; HRMS (FD-TOF) $\mathrm{m} / \mathrm{z} 2138.2445$ [M] ${ }^{+}$ (calcd for $\mathrm{C}_{147} \mathrm{H}_{171} \mathrm{~N}_{3} \mathrm{O}_{5} \mathrm{Se}, 2138.2383$ ).

\section{Reaction of selenol 3a with $\mathrm{H}_{2} \mathrm{O}_{2}$.}

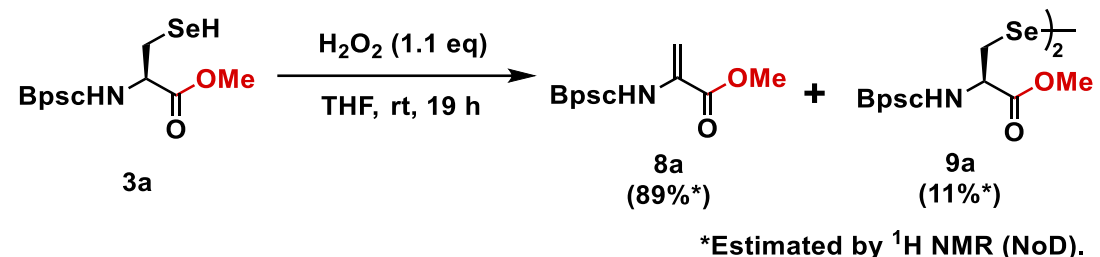

Scheme S13. Reaction of selenol 3a with $\mathrm{H}_{2} \mathrm{O}_{2}$ without any additives under neutral conditions.

A stock solution of $\mathrm{H}_{2} \mathrm{O}_{2}(30 \%, 15 \mu \mathrm{L}, 0.15 \mathrm{mmol})$ in THF $(2.0 \mathrm{~mL})$ was prepared prior to the reaction. Selenol 3a (14 mg, $6.9 \mu \mathrm{mol})$ was placed in a $10 \mathrm{~mL}$ Schlenk tube. After evacuated and backfilled with argon, THF (0.6 $\mathrm{mL})$ and the stock solution of $\mathrm{H}_{2} \mathrm{O}_{2}(75 \mathrm{mM}, 0.1 \mathrm{~mL}, 7.5 \mu \mathrm{mol})$ were added. Then the obtained solution was transferred to a J-young NMR tube. After $19 \mathrm{~h}$, a ${ }^{1} \mathrm{H}$ NMR (NoD) spectrum of the solution was recorded and the NMR yields of dehydroalanine $8 \mathbf{a}$ and diselenide $9 \mathbf{a}$ were determined as $89 \%$ and $11 \%$, respectively. The mixture was concentrated in vacuo and the crude product was separated by PTLC (hexane/ $\mathrm{CHCl}_{3}=1: 3$ ) to give 8a and 9a as colorless crystals and yellow crystals, respectively. 8a: yield $11 \mathrm{mg}(5.7 \mu \mathrm{mol}, 83 \%)$. 9a: yield $1.7 \mathrm{mg}(0.42$ $\mu \mathrm{mol}, 12 \%)$.

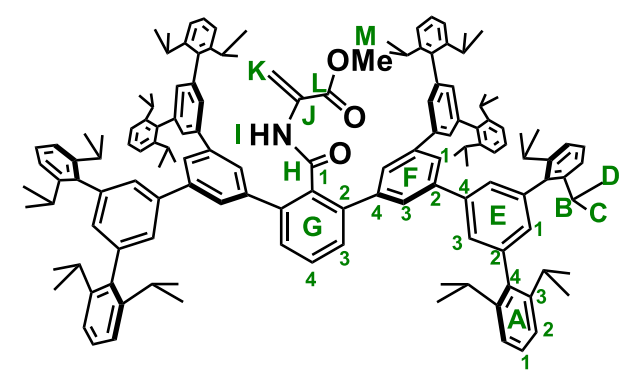

Dehydroalanine 8a: colorless crystals; m.p. $214-217^{\circ} \mathrm{C} .{ }^{1} \mathbf{H}$ NMR $\left(500 \mathrm{MHz}, \mathrm{CDCl}_{3}\right): \delta 1.01(\mathrm{~d}, J=6.8 \mathrm{~Hz}, 48 \mathrm{H}, \mathbf{C}$ or $\mathbf{D}), 1.08(\mathrm{~d}, J=$ $6.8 \mathrm{~Hz}, 48 \mathrm{H}, \mathbf{C}$ or D), 2.71 (septet, $J=6.8 \mathrm{~Hz}, 16 \mathrm{H}, \mathbf{B}), 3.24$ (s, $3 \mathrm{H}$, M), $4.95\left(\mathrm{~s}, 1 \mathrm{H}, \mathbf{K}_{\mathbf{A}}\right), 6.12\left(\mathrm{~s}, 1 \mathrm{H}, \mathbf{K}_{\mathbf{B}}\right), 6.96(\mathrm{br}, 4 \mathrm{H}, \mathbf{E} \mathbf{1}), 7.16(\mathrm{~d}, J=$ $7.7 \mathrm{~Hz}, 16 \mathrm{H}, \mathbf{A 2}), 7.30$ (t, $J=7.7 \mathrm{~Hz}, 8 \mathrm{H}, \mathbf{A 1}), 7.37$ (d, $J=1.7 \mathrm{~Hz}, 8 \mathrm{H}$, E3), 7.53 (br, 3H, G3 and G4), 7.67 (d, $J=1.7 \mathrm{~Hz}, 4 \mathrm{H}, \mathbf{F 3}), 7.76(\mathrm{br}$, $3 \mathrm{H}, \mathbf{F 1}$ and $\mathbf{I}) ;{ }^{13} \mathbf{C} \mathbf{N M R}\left(125 \mathrm{MHz}, \mathrm{CDCl}_{3}\right): \delta 24.3,24.5$ (q, $\mathbf{C}$ and $\left.\mathbf{D}\right)$, 30.5 (d, B), 52.6 (q, M), 108.2 (t, K), 122.6 (d, A2), 125.9 (d, F1), 126.7 (d, E3), 126.9 (d, F3), 128.0 (d, A1), 129.6 (d, G3), 130.1 (s, J), 130.3 (d, E1), 130.6 (d, G4), 135.5 (s, G1), 139.2 (s, A4), 140.2 (s, G2), 140.6 (s, F2), 141.0 (s, F4), 141.1 (s, E2), 142.0 (s, E4), 146.8 (s, A3), 163.5 (s, L), 168.0 (s, H); IR (KBr); 3394 (br), 3060, 3033, 2961, 2926, 2867, 1725, 1692, 1579, 1500, 1459, 1439, 1382, 1361, 1323, 1250, 1201, 1176, 1103, 1055, 901, 873, 805, 754, 725, 649, 631, 594, $586 \mathrm{~cm}^{-1}$; HRMS (FD-TOF) $\mathrm{m} / \mathrm{z}$ 1942.2623 [M] (calcd for $\mathrm{C}_{143} \mathrm{H}_{163} \mathrm{NO}_{3}, 1942.2632$ ).

Diselenide 9a: yellow crystals; m.p. $245-258{ }^{\circ} \mathrm{C} .{ }^{1} \mathbf{H}$ NMR $\left(500 \mathrm{MHz}, \mathrm{CDCl}_{3}\right): \delta 0.97(\mathrm{~d}, J=6.6 \mathrm{~Hz}, 96 \mathrm{H}, \mathbf{C}$ and D), 1.00-1.02 (m, 96H, C and D), $2.32\left(\mathrm{dd}, J=12.5,4.7 \mathrm{~Hz}, 2 \mathrm{H}, \mathbf{K}_{\mathbf{A}}\right), 2.38\left(\mathrm{dd}, J=12.9,6.0 \mathrm{~Hz}, 2 \mathrm{H}, \mathbf{K}_{\mathbf{B}}\right), 2.69$ (septet, $J=6.6 \mathrm{~Hz}, 32 \mathrm{H}, \mathbf{B}), 2.84$ (s, 6H, M), 4.06-4.09 (m, 2H, J), 5.88 (d, $J=8.3 \mathrm{~Hz}, 2 \mathrm{H}, \mathbf{I}), 6.93$ (br, 8H, E1), 7.09 (d, $J=7.7 \mathrm{~Hz}, 32 \mathrm{H}, \mathbf{A 2}), 7.22-7.26$ (m, 16H, A1), 7.40 (d, $J=1.2 \mathrm{~Hz}, 16 \mathrm{H}, \mathbf{E 3}), 7.44-7.45$ (m, 4H, G3), 7.497.52 (m, 2H, G4), 7.71 (br, 8H, F3), 7.76 (br, 4H, F1); $\left.{ }^{13} \mathbf{C ~ N M R ~ ( 1 2 5 ~ M H z , ~} \mathrm{CDCl}_{3}\right): \delta 24.2,24.3,24.45,24.48$ (q, 


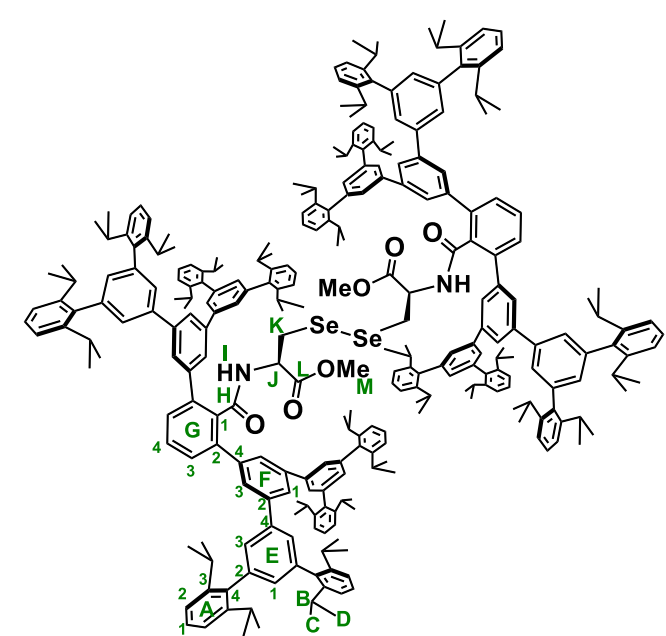

7.67; N, $0.70 \%$.
C and D), 30.5 (d, B, overlapped, t, K), 51.4 (q, M), 52.3 (d, J), 122.6 (d, A2), 126.0 (d, F1), 126.7 (d, E3), 127.5 (d, F3), 128.1 (d, A1), 129.4 (d, G4), 130.4 (d, E1), 130.5 (d, G3), 134.2 (s, G1), 139.1 (s, A4), 140.1 (s, G2), 140.6 (s, F2), 141.1 (s, E2), 141.4 (s, F4), 141.7 (s, E4), 146.75, 146.79 (s, A3), 167.8 (s, H), 169.2 (s, L); ${ }^{77} \mathbf{S e}$ NMR (95 MHz, CDCl $)_{3}$ : $\delta 296$; IR (KBr); 3420 (br), 3060, 3033, 2961, 2926, 2867, 1749, 1681, 1578, 1487, 1462, 1383, 1361, 1345, $1325,1250,1209,1178,1160,1054,873,827,805,754,725,713$ $\mathrm{cm}^{-1}$; HRMS (ESI-TOF) $\mathrm{m} / \mathrm{z} 2064.6804[\mathrm{M}+2 \mathrm{Na}]^{2+}$ (calcd for $\mathrm{C}_{286} \mathrm{H}_{328} \mathrm{~N}_{2} \mathrm{O}_{6} \mathrm{Se}_{2}$, $[\mathrm{M}+2 \mathrm{Na}]^{2+}$, 2046.6823). Anal. Calcd. for $\mathrm{C}_{286} \mathrm{H}_{328} \mathrm{~N}_{2} \mathrm{O}_{6} \mathrm{Se}: \mathrm{C}, 84.87 ; \mathrm{H}, 8.07$; N, 0.69. Found: C, 84.86; H,

Trapping of the transiently generated Sec-SeOH 1a by dimedone (10).

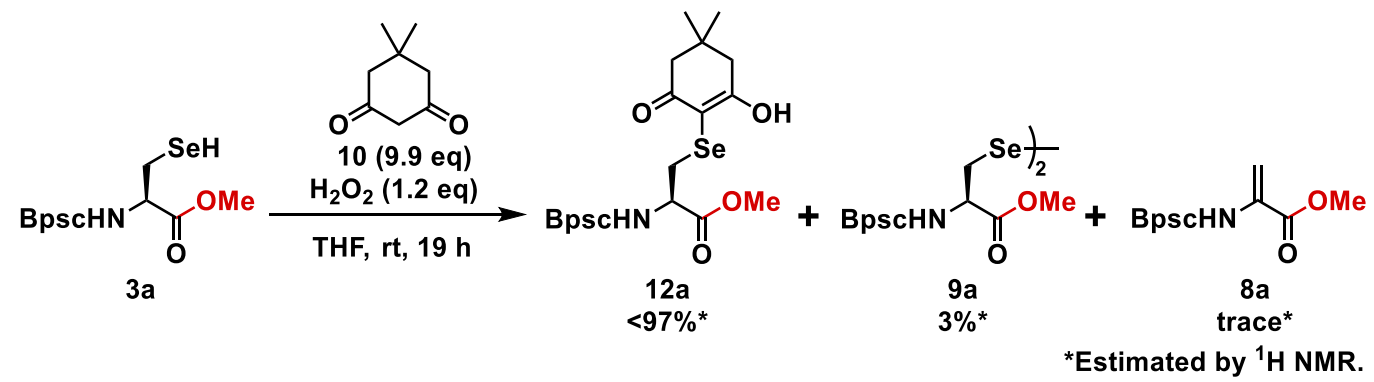

Scheme S14. Reaction of selenol 3a with $\mathrm{H}_{2} \mathrm{O}_{2}$ in the presence of dimedone (10) under neutral conditions.

A stock solution of $\mathrm{H}_{2} \mathrm{O}_{2}(30 \%, 2.5 \mu \mathrm{L}, 24 \mu \mathrm{mol})$ in THF (3.0 mL) was prepared prior to the reaction. Selenol 3a (12.5 mg, $6.17 \mu \mathrm{mol})$ and dimedone $(\mathbf{1 0} ; 8.5 \mathrm{mg}, 61 \mu \mathrm{mol}, 9.9 \mathrm{eq})$ were placed in a $10 \mathrm{~mL}$ Schlenk tube. After evacuated and backfilled with argon, the stock solution of $\mathrm{H}_{2} \mathrm{O}_{2}(8.0 \mathrm{mM}, 1.0 \mathrm{~mL}, 8.0 \mu \mathrm{mol})$ was added. The resulting solution was stirred at room temperature for $19 \mathrm{~h}$. After addition of water $(1 \mathrm{~mL})$, the two layers were separated, and the aqueous layer was extracted with $\mathrm{CHCl}_{3}(3 \times 4 \mathrm{~mL})$. The combined organic layer was dried over $\mathrm{Na}_{2} \mathrm{SO}_{4}$ and filtered. The filtrate was concentrated in vacuo. A ${ }^{1} \mathrm{H}$ NMR spectrum of the solution was recorded and the NMR yields of selenide 12a and diselenide 9a were determined as in $<97 \%$ and $3 \%$, respectively. The crude product was purified by PTLC (hexane/ $\left.\mathrm{CHCl}_{3}=1: 1\right)$ to give 12a as colorless crystals. Yield $10.3 \mathrm{mg}(4.8 \mu \mathrm{mol}$, $77 \%)$.

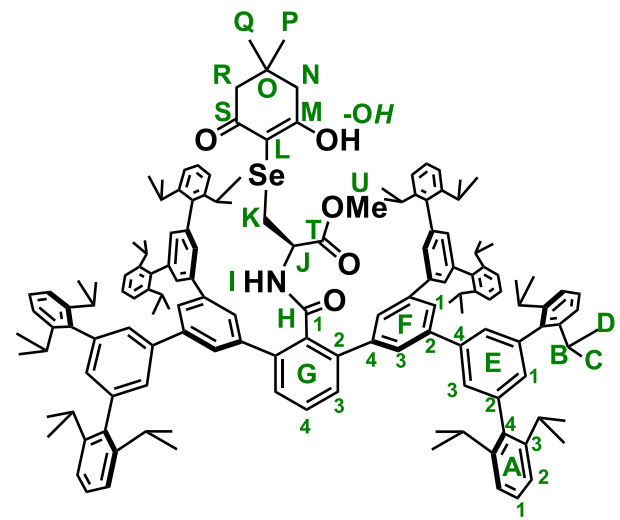

12a: colorless crystals, m.p. 218.0-220.0 ${ }^{\circ} \mathrm{C} .{ }^{1} \mathbf{H}$ NMR $(500 \mathrm{MHz}$, $\left.\mathrm{CDCl}_{3}\right): \delta 0.86$ (s, 3H, $\mathbf{Q}$ or $\left.\mathbf{P}\right), 0.87$ (s, $3 \mathrm{H}, \mathbf{Q}$ or $\left.\mathbf{P}\right), 1.02-1.03(\mathrm{~m}, 48 \mathrm{H}$, $\mathbf{C}$ and $\mathbf{D}), 1.07-1.10(\mathrm{~m}, 48 \mathrm{H}, \mathbf{C}$ and $\mathbf{D}), 1.93-2.09\left(\mathrm{~m}, 5 \mathrm{H}, \mathbf{K}_{\mathbf{A}}, \mathbf{N}\right.$ and R), $2.42\left(\mathrm{dd}, J=12.9,2.5 \mathrm{~Hz}, 1 \mathrm{H}, \mathbf{K}_{\mathbf{B}}\right), 2.69-2.79$ (m, 16H, B), 2.94 (s, $3 \mathrm{H}, \mathbf{U}), 4.18-4.22(\mathrm{~m}, 1 \mathrm{H}, \mathbf{J}), 6.64(\mathrm{~d}, J=8.0 \mathrm{~Hz}, 1 \mathrm{H}, \mathbf{I}), 6.97$ (br, 4H, E1), $7.16(\mathrm{~d}, J=7.7 \mathrm{~Hz}, 16 \mathrm{H}, \mathbf{A 2}), 7.31$ (t, $J=7.7 \mathrm{~Hz}, 8 \mathrm{H}, \mathbf{A 1}), 7.41$ (d, $J=1.2 \mathrm{~Hz}, 8 \mathrm{H}, \mathbf{E 3}), 7.41-7.43$ (m, 2H, G3), 7.47-7.50 (m, 1H, G4), $7.68(\mathrm{~d}, J=1.4 \mathrm{~Hz}, 4 \mathrm{H}, \mathbf{F 3}), 7.79$ (br, 2H, F1), 8.72 (br s, 1H, -OH); 
${ }^{13} \mathbf{C ~ N M R}\left(125 \mathrm{MHz}, \mathrm{CDCl}_{3}\right.$ ): $\delta$ 24.32, 24.38, 24.5 (q, C and D), 27.9 (q, $\mathbf{Q}$ or $\mathbf{P}$ ), 28.3 (q, $\mathbf{Q}$ or P), $30.4(\mathrm{t}, \mathbf{K}), 30.5$ (d, B), 31.4 (s, O), 42.4 (t, N), 50.9 (t, R), 52.3 (q, U), 52.7 (d, J), 102.8 (s, L), 122.6 (d, A2), 126.2 (d, F1), 126.7 (d, E3), 127.7 (d, F3), 128.0 (d, A1), 129.2 (d, G4), 130.1 (d, G3), 130.4 (d, E1), 134.7 (s, G1), 139.2 (s, A4), 140.4 (s, G2), 140.7 (s, F2), 141.1 (s, E2), 141.5 (s, F4), 141.7 (s, E4), 146.8, 146.9 (s, A3), 168.6 (s, H), 170.1 (s, T), 178.7 (s, M), 195.0 (s, S); ${ }^{77} \mathbf{S e}$ NMR (95 MHz, $\mathrm{CDCl}_{3}$ ): $\delta 57$ (br); IR (KBr); 3409 (br), 3272 (br), 3060, 3031, 2961, 2926, 2867, 1744, 1667, 1459, 1383, 1361, 1345, 1325, 1250, 1217, 1055, 873, 805, 754, $725 \mathrm{~cm}^{-1}$; HRMS (FD-TOF) $m / z 2162.2627[\mathrm{M}]^{+}$(calcd for $\mathrm{C}_{151} \mathrm{H}_{175} \mathrm{NO}_{5} \mathrm{Se}, 2162.2635$ ).

\section{Trapping of the transiently generated Sec-SeOH 1a with acetylacetone (11).}

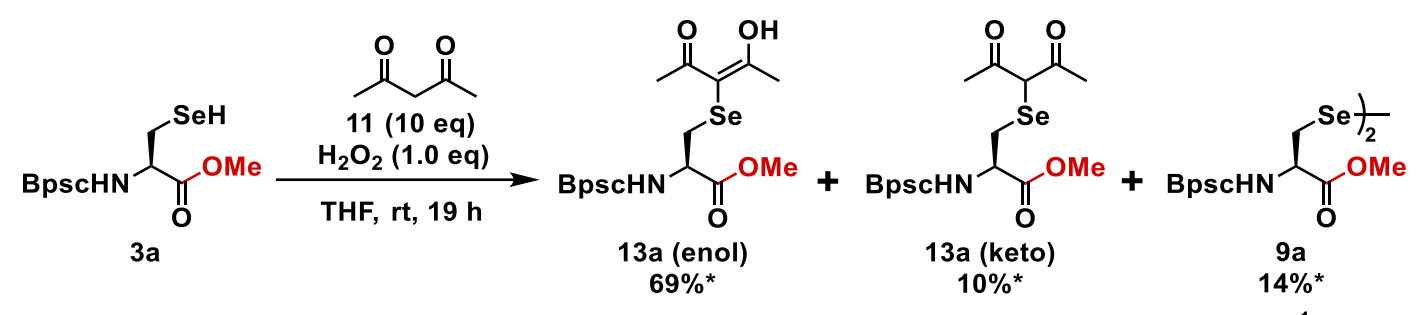

Scheme S15. Reaction of selenol 3a with $\mathrm{H}_{2} \mathrm{O}_{2}$ in the presence of acetyl acetone (11) under neutral conditions.

A stock solution of $\mathrm{H}_{2} \mathrm{O}_{2}(30 \%, 30 \mu \mathrm{L}, 0.29 \mathrm{mmol})$ in THF $(15.0 \mathrm{~mL})$ was prepared prior to the reaction. Selenol 3a $(21.0 \mathrm{mg}, 10.4 \mu \mathrm{mol})$ was placed in a $10 \mathrm{~mL}$ Schlenk tube. After evacuated and backfilled with argon, acetylacetone $(\mathbf{1 1} ; 11 \mu \mathrm{L}, 0.10 \mathrm{mmol}, 10 \mathrm{eq})$ and then the stock solution of $\mathrm{H}_{2} \mathrm{O}_{2}(19.3 \mathrm{mM}, 0.530 \mathrm{~mL}, 10.2 \mu \mathrm{mol})$ were added. The resulting solution was stirred at room temperature for $19 \mathrm{~h}$. The following workup was performed under argon atmosphere. After addition of degassed $10 \%$ aq. $\mathrm{Na}_{2} \mathrm{SO}_{3}(1.0 \mathrm{~mL})$, the two layers were separated, and the aqueous layer was extracted with $\mathrm{Et}_{2} \mathrm{O}(3 \times 4 \mathrm{~mL})$. The combined organic layers were dried over $\mathrm{Na}_{2} \mathrm{SO}_{4}$ and filtered. The filtrate was concentrated in vacuo. A ${ }^{1} \mathrm{H}$ NMR spectrum of the solution was recorded and the NMR yields of selenide 13a and diselenide 9a were determined as 79\% (keto/enol $=13: 87$ ) and 14\%, respectively. The crude product was purified by PTLC (hexane/ $\mathrm{CHCl}_{3}=1: 1$ ) to give the tautomeric mixture of 13a as colorless crystals. Yield $5.10 \mathrm{mg}(2.40 \mu \mathrm{mol}, 23 \%)$. Following data were collected for this mixture of keto/enol isomers.

13a $<$ keto/enol = 16:84>: colorless crystals, IR (KBr); 3419 (br), 3060, 3033, 2961, 2926, 2867, 1747, 1681, 1578, 1494, 1459, 1383, 1361, 1345, 1325, 1250, 1215, 1178, 1102, 1055, 873, 805, 754, $725 \mathrm{~cm}^{-1}$; HRMS (FD-TOF) $\mathrm{m} / z 2122.2320[\mathrm{M}]^{+}$(calcd for $\mathrm{C}_{148} \mathrm{H}_{171} \mathrm{NO}_{5} \mathrm{Se}, 2122.2322$ ). Anal. Calcd. for $\mathrm{C}_{148} \mathrm{H}_{171} \mathrm{NO}_{5} \mathrm{Se}: \mathrm{C}, 83.73 ; \mathrm{H}, 8.12 ; \mathrm{N}$, 0.66. Found: C, $83.64 ; \mathrm{H}, 8.26 ; \mathrm{N}, 0.67 \%$.

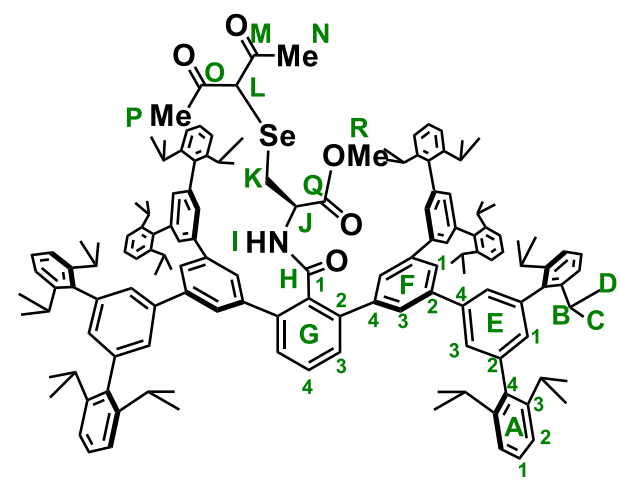

13a (keto form) : ${ }^{1} \mathbf{H}$ NMR $\left(500 \mathrm{MHz}, \mathrm{CDCl}_{3}\right): \delta 1.00-1.03(\mathrm{~m}, 48 \mathrm{H}$, $\mathbf{C}$ and $\mathbf{D}), 1.05-1.10$ (m, 48H, C and $\mathbf{D}), 1.86$ (s, 3H, $\mathbf{N}$ or $\mathbf{P}$ ), 1.89 (s, $3 \mathrm{H}, \mathbf{N}$ or $\mathbf{P}), 2.33\left(\mathrm{dd}, J=13.1,4.8 \mathrm{~Hz}, 1 \mathrm{H}, \mathbf{K}_{\mathbf{A}}\right), 2.44(\mathrm{dd}, J=13.2,4.8$ $\left.\mathrm{Hz}, 1 \mathrm{H}, \mathbf{K}_{\mathbf{B}}\right), 2.69-2.75$ (m, 16H, B), 3.05 (s, 3H, R), 3.86 (s, 1H, L), 4.35-4.38 (m, 1H, J), 6.63 (d, J=7.4 Hz, 1H, I), $6.96(\mathrm{br}, 4 \mathrm{H}, \mathbf{E 1}), 7.15-$ 7.17 (m, 16H, A2), 7.31 (t, $J=7.7 \mathrm{~Hz}, 8 \mathrm{H}, \mathbf{A 1}), 7.41$ (br, 8H, E3), 7.427.44 (m, 2H, G3), 7.50-7.53 (m, 1H, G4), 7.68 (br, 4H, F3), 7.81 (br, 2H, F1); ${ }^{13} \mathbf{C}$ NMR (125 MHz, $\left.\mathrm{CDCl}_{3}\right): \delta 24.2,24.3,24.4,24.5$ (q, C, 
D, and t, K), 27.8 (q, N or P), 28.1 (q, $\mathbf{N}$ or $\mathbf{P}$ ), 30.6 (d, B), 52.3 (d, $\mathbf{R}$ overlapped, d, $\mathbf{J}$ and a carbon $\mathbf{P}$ in enol form, determined by $H M B C$ ), 56.5 (s, L), 122.7 (d, A2), 125.7 (d, F1), 126.6 (d, E3), 127.2 (d, F3), 128.1 (d, A1), 129.5 (d, G4), 130.1 (d, G3), 130.5 (d, E1), 134.7 (s, G1), 139.2 (s, A4), 140.2 (s, G2), 140.5 (s, F2), 141.2 (s, E2), 141.6 (s, E4), 141.7 (s, F4), 146.8 (s, A3), 168.7 (s, H), 170.1 (s, Q, overlapped with a carbon $\mathbf{O}$ in enol form, determined by $H M B C$ ), 200.0 (s, M and O); ${ }^{77} \mathbf{S e} \mathbf{N M R}\left(95 \mathrm{MHz}, \mathrm{CDCl}_{3}\right.$ ): $\delta 263$.

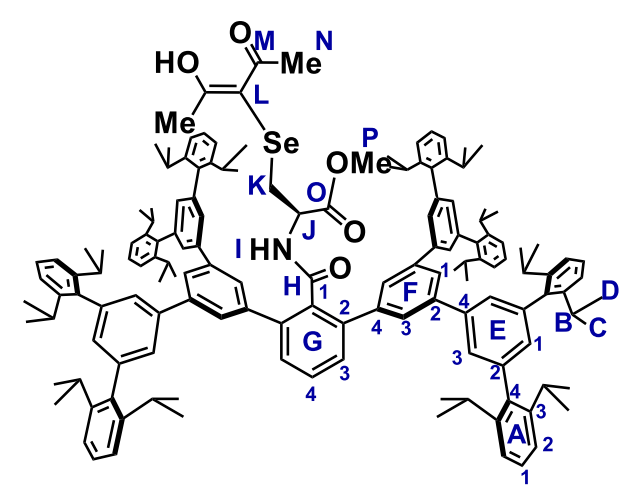

13a (enol form) : ${ }^{1} \mathbf{H}$ NMR (500 MHz, $\left.\mathrm{CDCl}_{3}\right): \delta 1.00-1.03$ (m, $48 \mathrm{H}$, C and D), 1.05-1.10 (m, 48H, C and D), $1.96(\mathrm{~s}, 6 \mathrm{H}, \mathbf{N}), 2.26(\mathrm{dd}, J=$ 13.2, 4.8 Hz, 1H, $\left.\mathbf{K}_{\mathbf{A}}\right), 2.64-2.67\left(\mathrm{~m}, 1 \mathrm{H}, \mathbf{K}_{\mathbf{B}}\right), 2.69-2.75(\mathrm{~m}, 16 \mathrm{H}, \mathbf{B})$, 2.75 (s, 3H, P), 4.58-4.61 (m, 1H, J), 6.28 (d, J = 7.4 Hz, 1H, I), 6.97 (br, 4H, E1), 7.15-7.17 (m, 16H, A2), 7.31 (t, $J=7.7 \mathrm{~Hz}, 8 \mathrm{H}, \mathbf{A 1}), 7.41$ (br, 8H, E3), 7.42-7.44 (m, 2H, G3), 7.48-7.51 (m, 1H, G4), 7.67 (br, 4H, F3), 7.79 (br, 2H, F1); ${ }^{13} \mathbf{C}$ NMR (125 MHz, $\left.\mathrm{CDCl}_{3}\right)$ : $\delta$ 24.2, 24.3, 24.4, 24.5 (q, C and D), 25.7 (q, N), 28.3 (t, K), 30.6 (d, B), 50.6 (d, J), 52.3 (q, $\mathbf{P}$, overlapped with a carbon $\mathbf{R}$ and $\mathbf{J}$ in keto form, determined by HMBC), 97.2 (s, L), 122.7 (d, A2), 126.1 (d, F1), 126.6 (d, E3), 127.1 (d, F3), 128.1 (d, A1), 129.4 (d, G4), 130.2 (d, G3), 130.5 (d, E1), 134.9 (s, G1), 139.2 (s, A4), 140.2 (s, G2), 140.5 (s, F2), 141.2 (s, E2), 141.85 (s, F4), 141.88 (s, E4), 146.8 (s, A3), 167.9 (s, H), 170.1 (s, O, overlapped with a carbon $\mathbf{Q}$ in keto form), 196.6 (s, M); ${ }^{77} \mathrm{Se}$ NMR $\left(95 \mathrm{MHz}, \mathrm{CDCl}_{3}\right): \delta 146$.

Direct observation of Sec-SeOH 1a in the oxidation of selenol 3a by ${ }^{1} \mathrm{H}$ NMR spectroscopy.

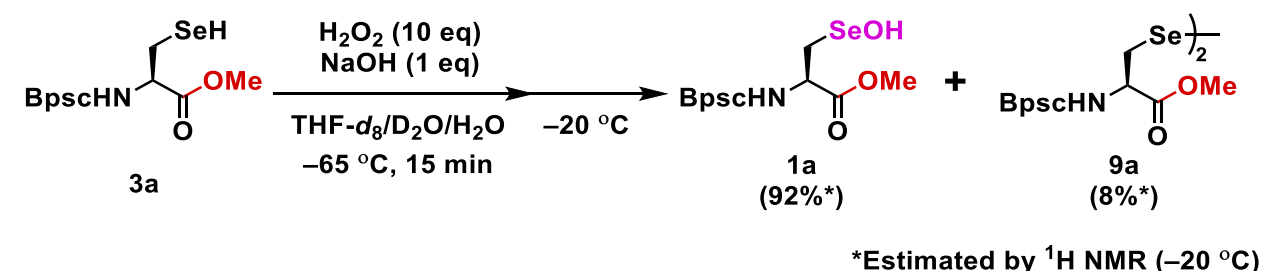

Scheme S16. Direct observation of $\mathrm{Sec}-\mathrm{SeOH} 1 \mathrm{a}$.

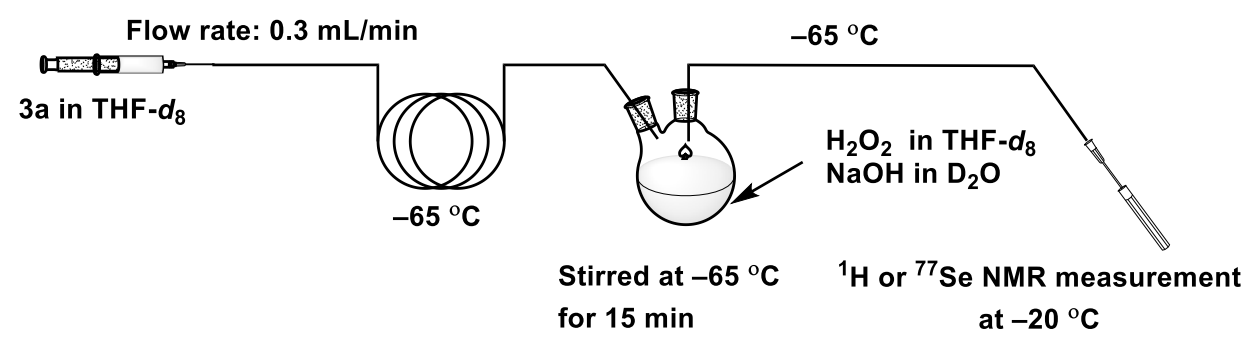

Figure S1. Protocol for the direct observation of $\mathrm{Sec}-\mathrm{SeOH} 1 \mathrm{a}$.

All solvents were degassed by argon bubbling before use. A stock solution of $\mathrm{H}_{2} \mathrm{O}_{2}(30 \%, 80 \mathrm{~mL}, 0.78 \mathrm{mmol})$ in THF- $d_{8}(1.0 \mathrm{~mL})$ and that of $\mathrm{NaOH}(93 \%$ purity, $102.6 \mathrm{mg}, 2.38 \mathrm{mmol})$ in $\mathrm{D}_{2} \mathrm{O}(1.00 \mathrm{~mL})$ were prepared prior to the reaction. After evacuated and backfilled with argon, THF- $d_{8}(0.50 \mathrm{~mL})$, the stock solution of $\mathrm{NaOH}(2.38 \mathrm{M}$, $2.6 \mathrm{~mL}, 6.2 \mathrm{mmol})$, and then that of $\mathrm{H}_{2} \mathrm{O}_{2}(0.78 \mathrm{M}, 71 \mathrm{~mL}, 55 \mathrm{mmol})$ were added and the obtained mixture was cooled to $-65^{\circ} \mathrm{C}$. After the flask was set in the system shown in Figure S1, selenol 3a (11.2 mg, $\left.5.53 \mathrm{mmol}\right)$ and 1 
$\mu \mathrm{L}$ of bis(trimethylsilyl)methane $\left(\mathrm{Me}_{3} \mathrm{SiCH}_{2} \mathrm{SiMe}_{3}\right)$ as an internal standard in THF- $d_{8}(0.40 \mathrm{~mL})$ was added dropwise via a cooled tube $\left(-65^{\circ} \mathrm{C}\right)$ to the solution prepared as described by using a syringe pump (flow rate: 0.3 $\mathrm{mL} / \mathrm{min}$ ). The obtained solution was stirred at $-65^{\circ} \mathrm{C}$ for $15 \mathrm{~min}$ and then transferred to a J-young NMR tube via a cooled tube $\left(-65^{\circ} \mathrm{C}\right)$ carefully. The formation of $\mathrm{Sec}-\mathrm{SeOH} 1 \mathrm{a}$ and diselenide 9a in 92\% and 8\% NMR yields, respectively, were observed by ${ }^{1} \mathrm{H}$ NMR spectroscopy at $-20{ }^{\circ} \mathrm{C}$. (Figure S2 and S3). Comparison of the ${ }^{1} \mathrm{H}$ NMR chemical shifts $\mathbf{3 a}$ and $\mathbf{1 a}$ is shown in Table $\mathrm{S} 1$.

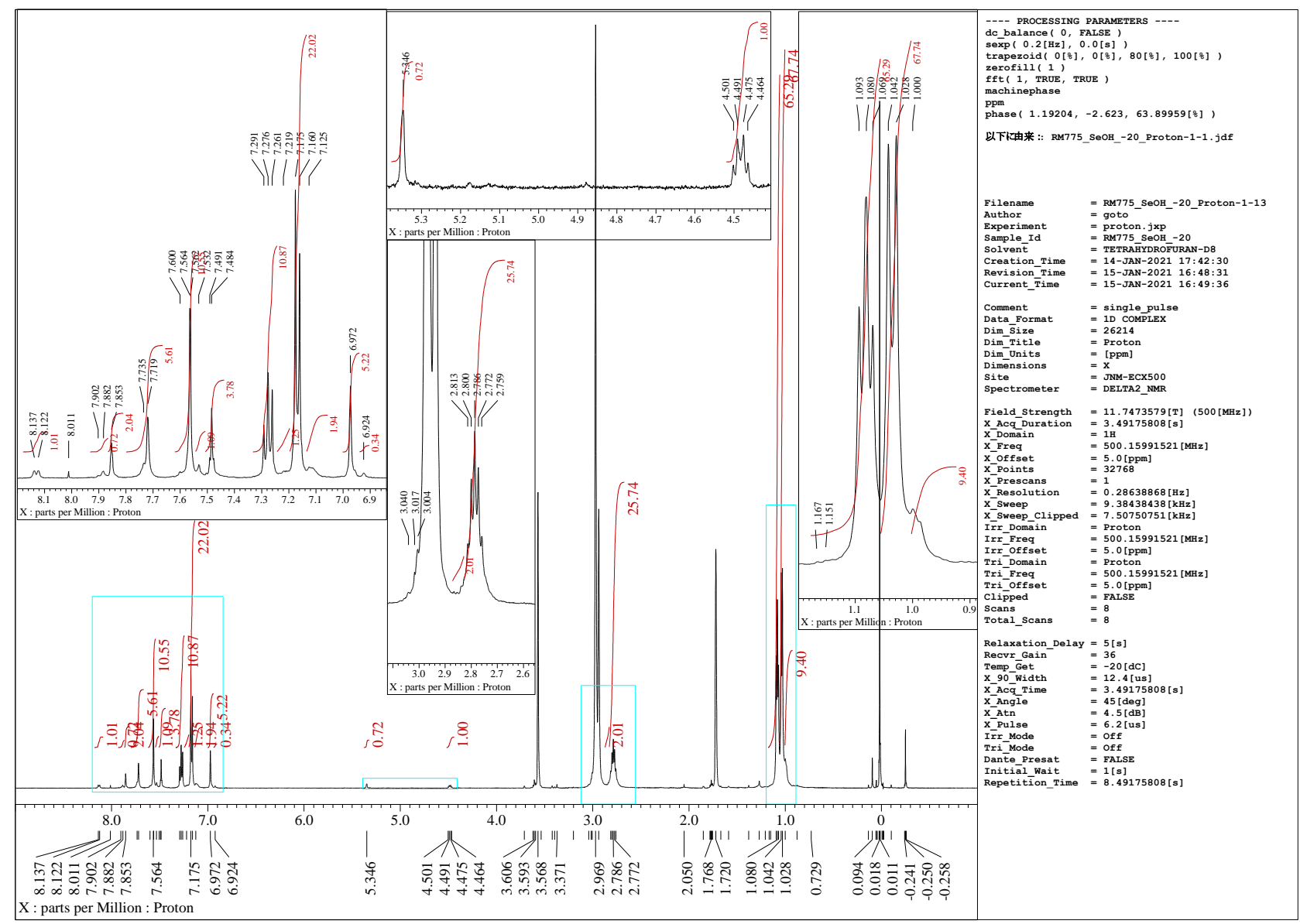

Figure S2. ${ }^{1} \mathrm{H}$ NMR $\left(500 \mathrm{MHz}, \mathrm{THF}-d_{8},-20{ }^{\circ} \mathrm{C}\right)$ spectrum of the mixture obtained in Scheme S16.

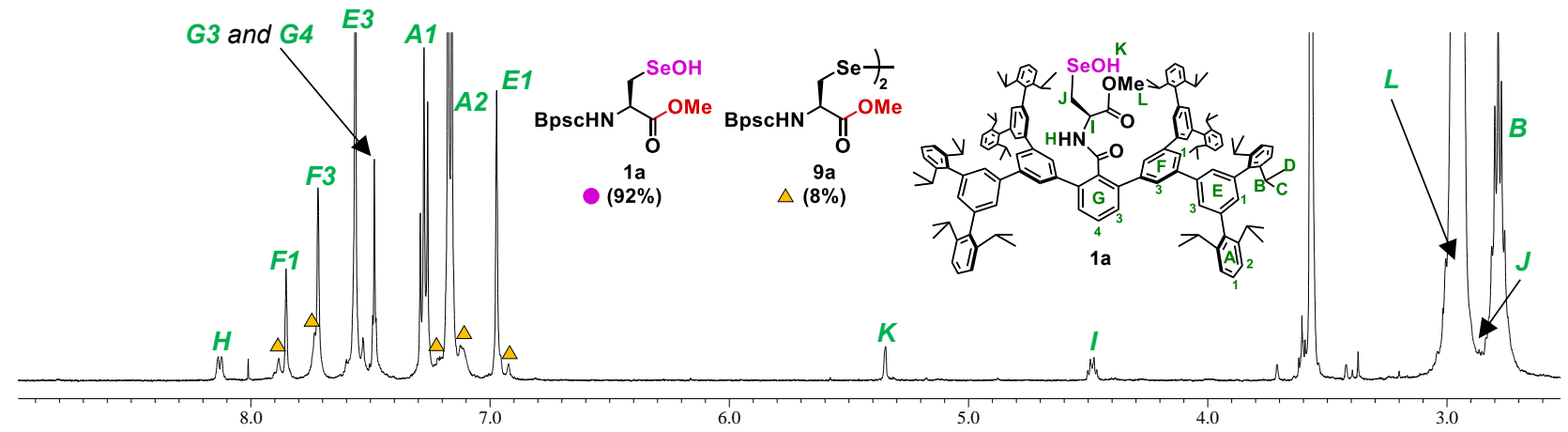

Figure S3. Assignment of the signal in ${ }^{1} \mathrm{H}$ NMR $\left(500 \mathrm{MHz}\right.$, THF- $\left.-d_{8},-20{ }^{\circ} \mathrm{C}\right)$ spectrum of the mixture obtained in Scheme S16. 
Table S1. Comparison of the chemical shifts of 3a and $1 \mathbf{a}$ on ${ }^{1} \mathrm{H}$ NMR $\left(500 \mathrm{MHz}, \mathrm{THF}-d_{8},-20{ }^{\circ} \mathrm{C}\right)$.

\begin{tabular}{cccc}
\hline & \multirow{2}{*}{$\begin{array}{c}\text { BpscHN } \\
\text { methine proton }(\mathrm{ppm})\end{array}$} & $-\mathrm{N} H-(\mathrm{ppm})$ & \\
\hline $\mathbf{3 a}(\mathrm{X}=\mathrm{H})+\mathrm{NaOH}(1 \mathrm{eq})$ & $4.53-4.62(\mathrm{~m})$ & $7.99(\mathrm{~d}, J=5.5 \mathrm{~Hz})$ & $-1.43(\mathrm{br} \mathrm{t}, J=8.1 \mathrm{~Hz})$ \\
\hline $\mathbf{1 a}(\mathrm{X}=\mathrm{OH})$ & $4.48(\mathrm{td}, J=7.4,5.3 \mathrm{~Hz})$ & $8.13(\mathrm{~d}, J=7.4 \mathrm{~Hz})$ & $5.35(\mathrm{~s})$ \\
\hline
\end{tabular}

Direct observation of Sec-SeOH 1a by ${ }^{77} \mathrm{Se}$ NMR spectroscopy.

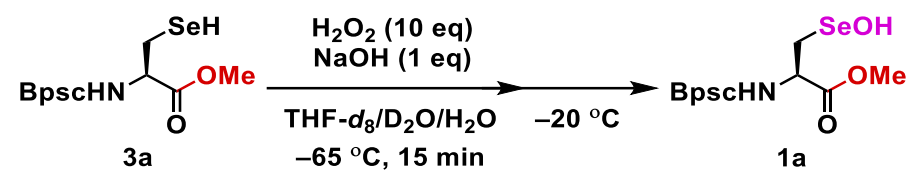

Scheme S17. Direct observation of Sec-SeOH 1a by ${ }^{77} \mathrm{Se}$ NMR spectroscopy.

All solvents were degassed by argon bubbling before use. A stock solution of $\mathrm{NaOH}$ (93\% purity, $92.7 \mathrm{mg}, 2.16$ $\mathrm{mmol})$ in $\mathrm{D}_{2} \mathrm{O}(0.5 \mathrm{~mL})$ was prepared prior to the reaction. After evacuated and backfilled with argon, THF- $d_{8}(0.3$ $\mathrm{mL})$, the stock solution of $\mathrm{NaOH}(4.3 \mathrm{M}, 9.0 \mu \mathrm{L}, 40 \mu \mathrm{mol})$ and then $\mathrm{H}_{2} \mathrm{O}_{2}(30 \%, 41 \mu \mathrm{L}, 0.40 \mathrm{mmol})$ were added and the obtained mixture was cooled to $-65^{\circ} \mathrm{C}$. After the flask was set in the system shown in Figure S1, selenol 3a $(83.9 \mathrm{mg}, 41.4 \mu \mathrm{mol})$ in THF- $d_{8}(0.4 \mathrm{~mL})$ was added dropwise via a cooled tube $\left(-65^{\circ} \mathrm{C}\right)$ to the solution prepared as described above by using a syringe pump (flow rate: $0.3 \mathrm{~mL} / \mathrm{min}$ ). The obtained solution was stirred at $-65^{\circ} \mathrm{C}$ for $15 \mathrm{~min}$ and then transferred to a J-young NMR tube via a cooled tube $\left(-65^{\circ} \mathrm{C}\right)$ carefully. $\mathrm{A}^{77} \mathrm{Se}$ NMR spectrum was recorded at $-20^{\circ} \mathrm{C}$ (Figure S4). A signal of $1 \mathbf{a}$ was observed at $1298 \mathrm{ppm}$.

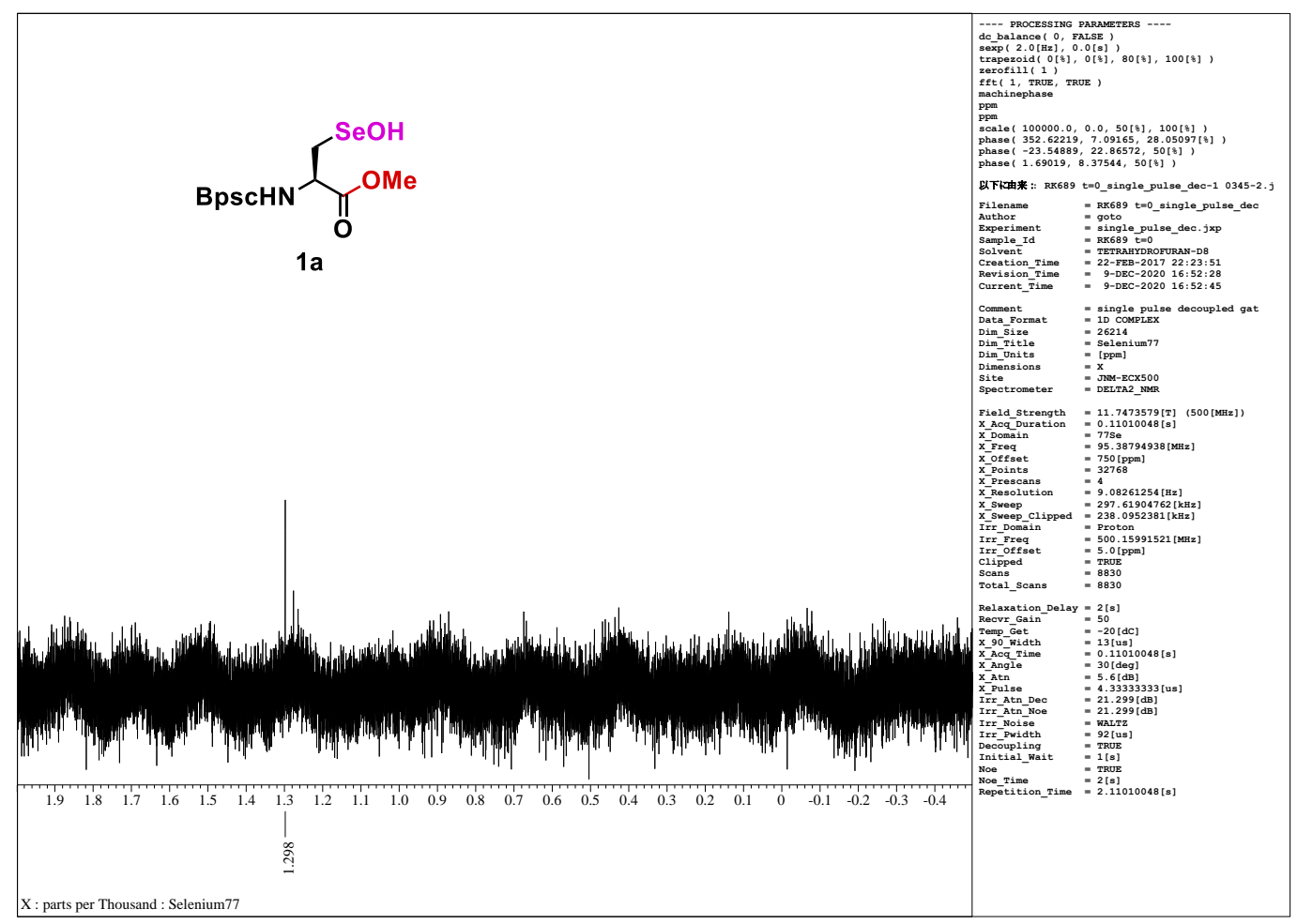

Figure S4. ${ }^{77} \mathrm{Se}$ NMR $\left(95 \mathrm{MHz}, \mathrm{THF}-d_{8},-20{ }^{\circ} \mathrm{C}\right)$ spectrum of the mixture obtained in Scheme S17. 


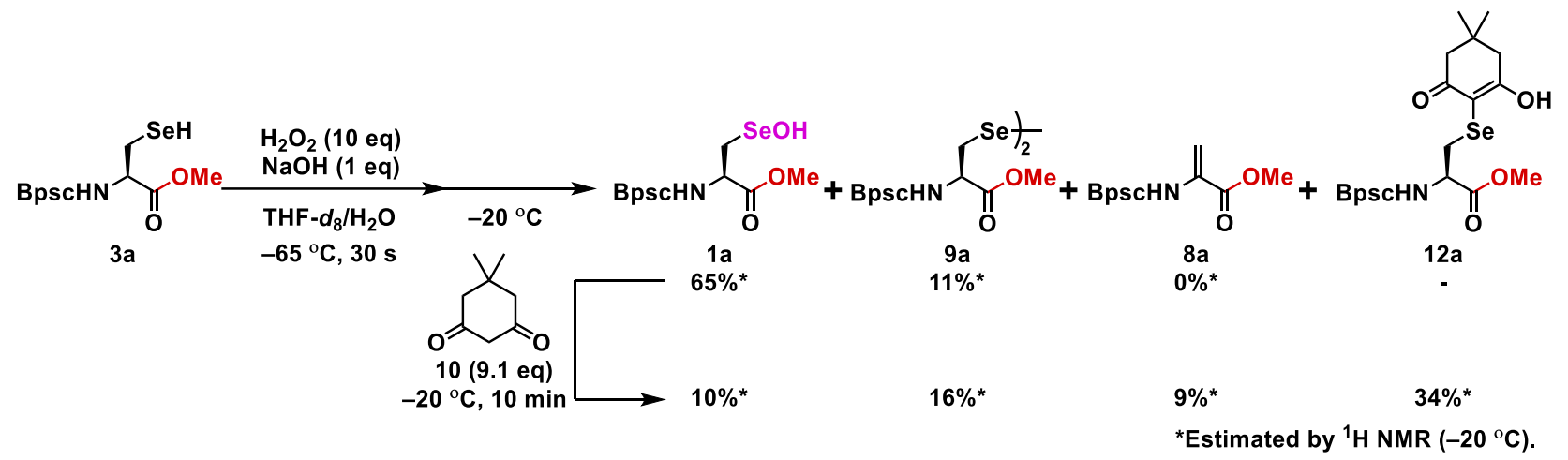

Scheme S18. Direct observation of 1a and its reaction with dimedone (10).

All solvents were degassed by argon bubbling before use. A stock solution of $\mathrm{NaOH}$ (93\% purity, $92 \mathrm{mg}, 2.1$ $\mathrm{mmol})$ in $\mathrm{H}_{2} \mathrm{O}(1.0 \mathrm{~mL})$ was prepared prior to the reaction. After evacuated and backfilled with argon, THF- $d_{8}(0.45$ $\mathrm{mL})$, the stock solution of $\mathrm{NaOH}(2.1 \mathrm{M}, 3.1 \mu \mathrm{L}, 6.6 \mu \mathrm{mol})$, and then $\mathrm{H}_{2} \mathrm{O}_{2}(30 \%, 7.2 \mu \mathrm{L}, 70 \mu \mathrm{mol})$ were added and the obtained mixture was cooled to $-65^{\circ} \mathrm{C}$. After the flask was set in the system shown in Figure S1, selenol 3a (14.6 mg, $7.21 \mu \mathrm{mol})$ and $1 \mu \mathrm{L}$ of bis(trimethylsilyl)methane as an internal standard in THF- $d_{8}(0.4 \mathrm{~mL})$ was added dropwise via a cooled tube $\left(-65^{\circ} \mathrm{C}\right)$ to the solution prepared as described above by using a syringe pump (flow rate: $0.9 \mathrm{~mL} / \mathrm{min}$ ). The obtained solution was stirred at $-65^{\circ} \mathrm{C}$ for $30 \mathrm{sec}$ and then transferred to a J-young NMR tube via a cooled tube $\left(-65{ }^{\circ} \mathrm{C}\right)$ carefully. A ${ }^{1} \mathrm{H}$ NMR spectrum was recorded at $-20^{\circ} \mathrm{C}$. The formation of $\mathrm{Sec}-\mathrm{SeOH} 1 \mathrm{a}$ and diselenide 9a were observed in 65\% and 11\% yields, respectively. After 10 min, dimedone (10; $9.2 \mathrm{mg}, 66$ $\mu$ mol, 9.1 eq) was added to the mixture at $-55^{\circ} \mathrm{C}$. After $10 \mathrm{~min}$, a ${ }^{1} \mathrm{H}$ NMR spectrum was recorded again at $-20{ }^{\circ} \mathrm{C}$. The formation of selenide 12a, dehydroalanine 8a, diselenide 9a, and Sec-SeOH 1a were observed in 34\%, 9\%, $16 \%$, and $10 \%$ yields, respectively. A dimedone unit of 12a was found to adopt only enol form. The signals of 12a in ${ }^{1} \mathrm{H}$ NMR spectrum were in agreement with those of purified 12a.

\section{Derivatization of Sec-SeOH 1a with acetylacetone (11).}

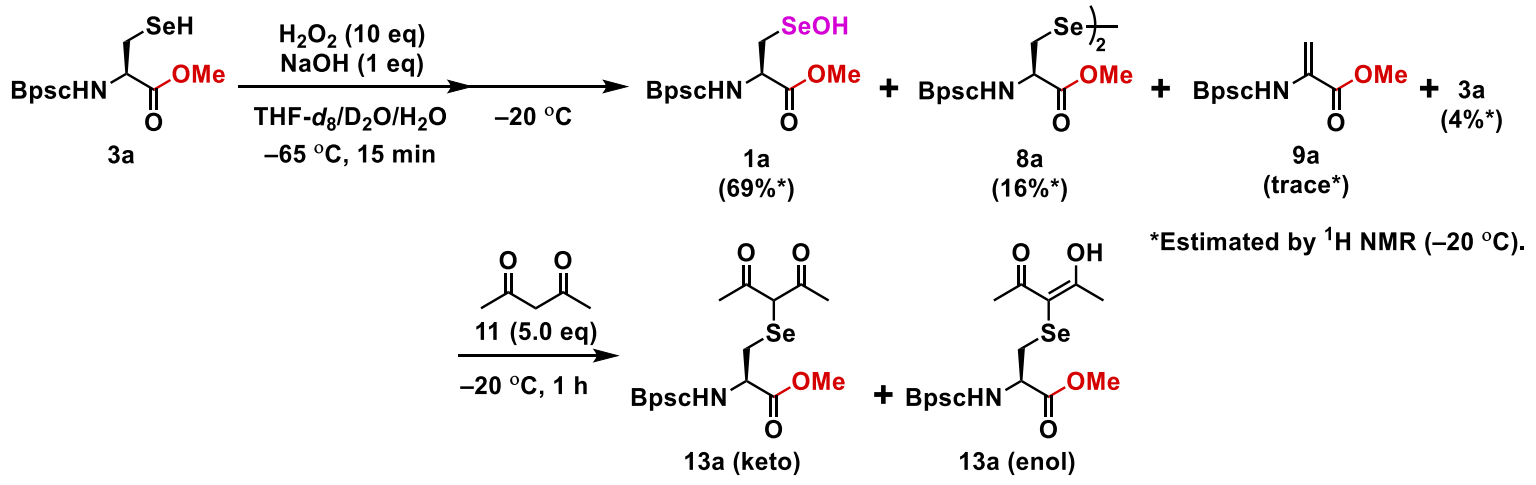

Scheme S19. Direct observation of 1a and its reaction with acetyl acetone (11).

All solvents were degassed by argon bubbling before use. A stock solution of $\mathrm{H}_{2} \mathrm{O}_{2}(30 \%, 80 \mu \mathrm{L}, 0.78 \mathrm{mmol})$ in THF- $d_{8}(0.5 \mathrm{~mL})$ and that of $\mathrm{NaOH}(93 \%$ purity, $96 \mathrm{mg}, 2.2 \mathrm{mmol})$ in $\mathrm{D}_{2} \mathrm{O}(1.0 \mathrm{~mL})$ were prepared prior to the reaction. After evacuated and backfilled with argon, THF- $d_{8}(0.4 \mathrm{~mL})$, the stock solution of $\mathrm{NaOH}(2.2 \mathrm{M}, 3.0 \mu \mathrm{L}$, $6.0 \mu \mathrm{mol})$ and then that of $\mathrm{H}_{2} \mathrm{O}_{2}(1.3 \mathrm{M}, 44 \mu \mathrm{L}, 59 \mu \mathrm{mol})$ were added and the obtained mixture was cooled to $-65{ }^{\circ} \mathrm{C}$. 
After the flask was set in the system shown in Figure S1, selenol 3a $(12.3 \mathrm{mg}, 6.12 \mu \mathrm{mol})$ and $1 \mu \mathrm{L}$ of bis(trimethylsilyl)methane as an internal standard in THF- $d_{8}(0.4 \mathrm{~mL})$ was added dropwise via a cooled tube $\left(-65^{\circ} \mathrm{C}\right)$ to the solution prepared as described above by using a syringe pump (flow rate: $0.3 \mathrm{~mL} / \mathrm{min}$ ). The obtained solution was stirred at $-65^{\circ} \mathrm{C}$ for $15 \mathrm{~min}$ and then transferred to a J-young NMR tube via a cooled tube $\left(-65^{\circ} \mathrm{C}\right)$ carefully. A ${ }^{1} \mathrm{H}$ NMR spectrum was recorded at $-20^{\circ} \mathrm{C}$. Sec-SeOH 1a, diselenide 9a, and remained selenol 3a were observed in 69\%, 16\%, and 4\% NMR yields, respectively. After recording the NMR spectrum, acetylacetone (11; $3.0 \mu \mathrm{L}, 29 \mu \mathrm{mol}, 5.0 \mathrm{eq}$ ) was added at $-65^{\circ} \mathrm{C}$. After $10 \mathrm{~min}$, the change of the components depending on the time was monitored by ${ }^{1} \mathrm{H}$ NMR spectroscopy at $-20{ }^{\circ} \mathrm{C}$ (Table S2, Figure S5).

Table S2. Monitoring of the composition of the solution obtained in Scheme S19 after addition of $\mathbf{1 1}$ by ${ }^{1} \mathrm{H}$ NMR $\left(500 \mathrm{MHz}, \mathrm{THF}-d_{8},-20^{\circ} \mathrm{C}\right)$ spectroscopies.

\begin{tabular}{|c|c|c|c|c|c|}
\hline \multirow[b]{2}{*}{ time (min) } & \multicolumn{4}{|c|}{ NMR yield (\%) } & \multirow[b]{2}{*}{ keto/enol ratio } \\
\hline & $1 \mathrm{a}$ & $8 a$ & $9 a$ & $13 a$ & \\
\hline 4 & 38 & 2 & 17 & 45 & $71: 29$ \\
\hline 11 & 11 & 2 & 17 & 64 & $64: 36$ \\
\hline 19 & 5 & 4 & 17 & 70 & $61: 39$ \\
\hline 53 & 0 & 6 & 17 & 67 & $51: 49$ \\
\hline
\end{tabular}

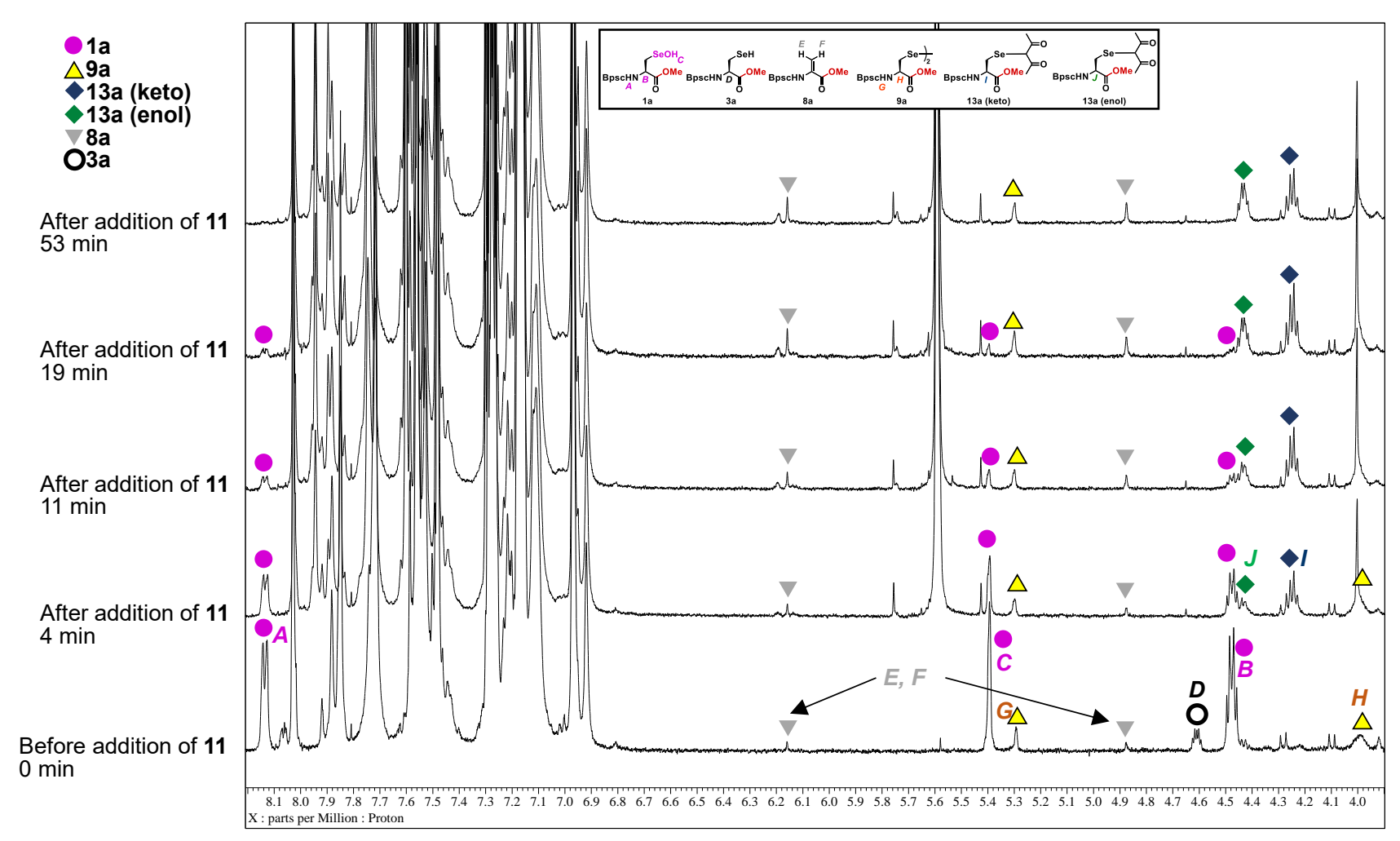

Figure S5. Monitoring of the mixture obtained in Scheme S19 by ${ }^{1} \mathrm{H}$ NMR $\left(500 \mathrm{MHz}\right.$, THF- $\left.d_{8},-20{ }^{\circ} \mathrm{C}\right)$ spectroscopies. 
Thermal decomposition of Sec-SeOH 1a.

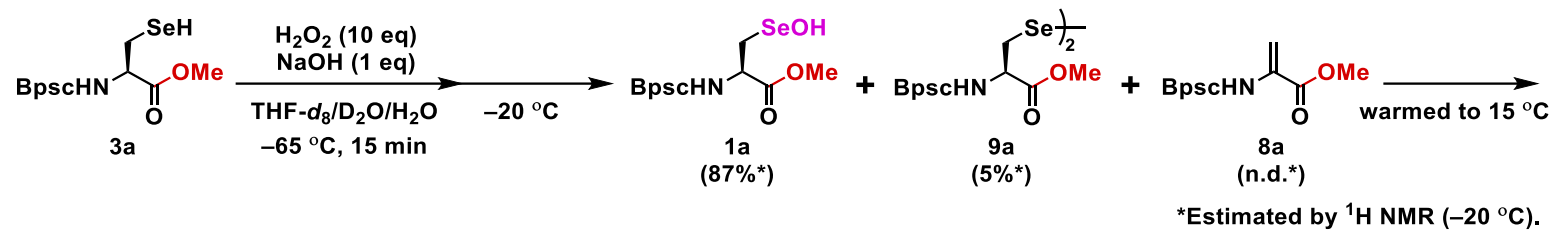

Scheme S20. Direct observation of 1a and thermal deselenation to 8a.

All solvents were degassed by argon bubbling before use. A stock solution of $\mathrm{NaOH}$ (93\% purity, $95.8 \mathrm{mg}, 2.23$ $\mathrm{mmol})$ in $\mathrm{D}_{2} \mathrm{O}(0.5 \mathrm{~mL})$ was prepared prior to the reaction. After evacuated and backfilled with argon, THF- $d_{8}(0.6$ $\mathrm{mL})$, a stock solution of $\mathrm{NaOH}(4.4 \mathrm{M}, 2.3 \mu \mathrm{L}, 10 \mu \mathrm{mol})$, and then that of $\mathrm{H}_{2} \mathrm{O}_{2}(30 \%, 10 \mu \mathrm{L}, 98 \mu \mathrm{mol})$ were added and the obtained mixture was cooled to $-65^{\circ} \mathrm{C}$. After the flask was set in the system shown in Figure S1, selenol 3a $(21.6 \mathrm{mg}, 10.7 \mu \mathrm{mol})$ and $2 \mu \mathrm{L}$ of bis(trimethylsilyl)methane as an internal standard in THF- $d_{8}(0.8 \mathrm{~mL})$ was added dropwise via a cooled tube $\left(-65^{\circ} \mathrm{C}\right)$ to the solution prepared as described above by using a syringe pump (flow rate: $0.3 \mathrm{~mL} / \mathrm{min}$ ). The obtained solution was stirred at $-65^{\circ} \mathrm{C}$ for $15 \mathrm{~min}$ and then transferred to a J-young NMR tube via a cooled tube $\left(-65^{\circ} \mathrm{C}\right)$ carefully. A ${ }^{1} \mathrm{H}$ NMR spectrum was recorded at $-20{ }^{\circ} \mathrm{C}$. The formation of $\mathrm{Sec}-\mathrm{SeOH}$ 1a and diselenide 9a were observed in $87 \%$ and 5\% yields, respectively. The obtained solution was warmed to $15^{\circ} \mathrm{C}$ and the ${ }^{1} \mathrm{H}$ NMR spectra were recorded at the temperatures shown in Table S3.

Table S3. Monitoring of the composition of the solution obtained in Scheme S20.

\begin{tabular}{ccccc} 
& & \multicolumn{3}{c}{ NMR yield (\%) } \\
\cline { 3 - 5 } time (min) & temp. $\left({ }^{\circ} \mathrm{C}\right)$ & $\mathbf{1 a}$ & $\mathbf{8 a}$ & $\mathbf{9 a}$ \\
0 & -20 & 87 & n.d. & 5 \\
5 & -10 & 79 & 2 & 6 \\
10 & 0 & 83 & 3 & 5 \\
15 & 5 & 55 & 16 & 7 \\
20 & 10 & 12 & 54 & 7 \\
25 & 15 & n.d. & 73 & 7
\end{tabular}

Reaction of Sec-SeOH 1a with cysteine thiol 6.
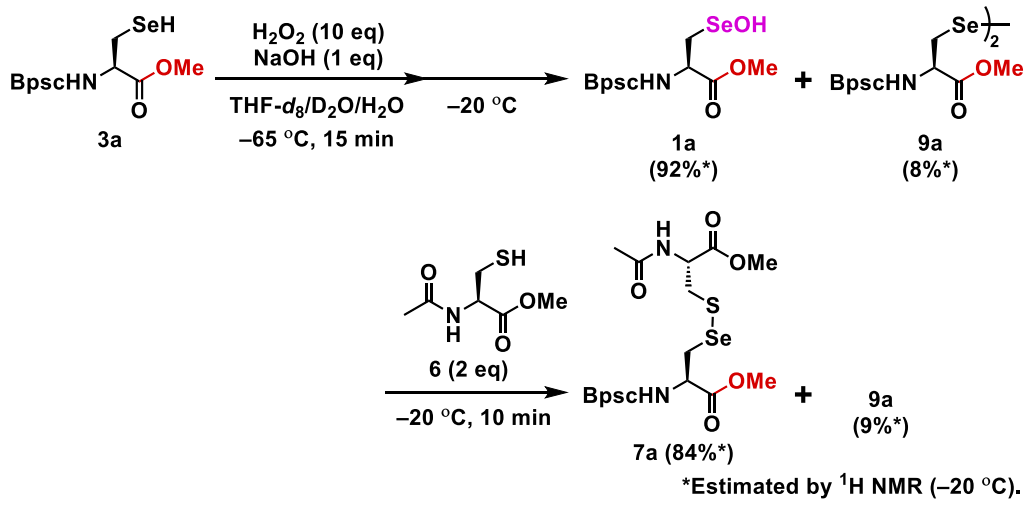

Scheme S21. Direct observation of Sec-SeOH 1a and its reaction with cysteine thiol 6. 
The mixture which was obtained in Scheme S16 was used for this experiment. After recording the ${ }^{1} \mathrm{H}$ NMR spectrum at $-20{ }^{\circ} \mathrm{C}$, a solution of 6 in THF- $d_{8}(0.10 \mathrm{M}, 111 \mu \mathrm{L}, 11 \mu \mathrm{mol})$ was added to the above mixture at $-65{ }^{\circ} \mathrm{C}$. After $8 \mathrm{~min}, \mathrm{a}{ }^{1} \mathrm{H}$ NMR spectrum of the obtained solution was recorded at $-20^{\circ} \mathrm{C}$. Disappearance of $\mathrm{Sec}-\mathrm{SeOH} \mathbf{1 a}$ and formation of the corresponding selenenyl sulfide 7a and diselenide 9a in 84\% and 9\% NMR yields, respectively, were observed by ${ }^{1} \mathrm{H}$ NMR spectroscopy at $-20{ }^{\circ} \mathrm{C}$. The signals of $7 \mathbf{a}$ in ${ }^{1} \mathrm{H}$ NMR were in agreement with those of the isolated sample. A comparison of the ${ }^{1} \mathrm{H}$ NMR spectra before and after the addition of thiol 6 is shown below (Figure S6).

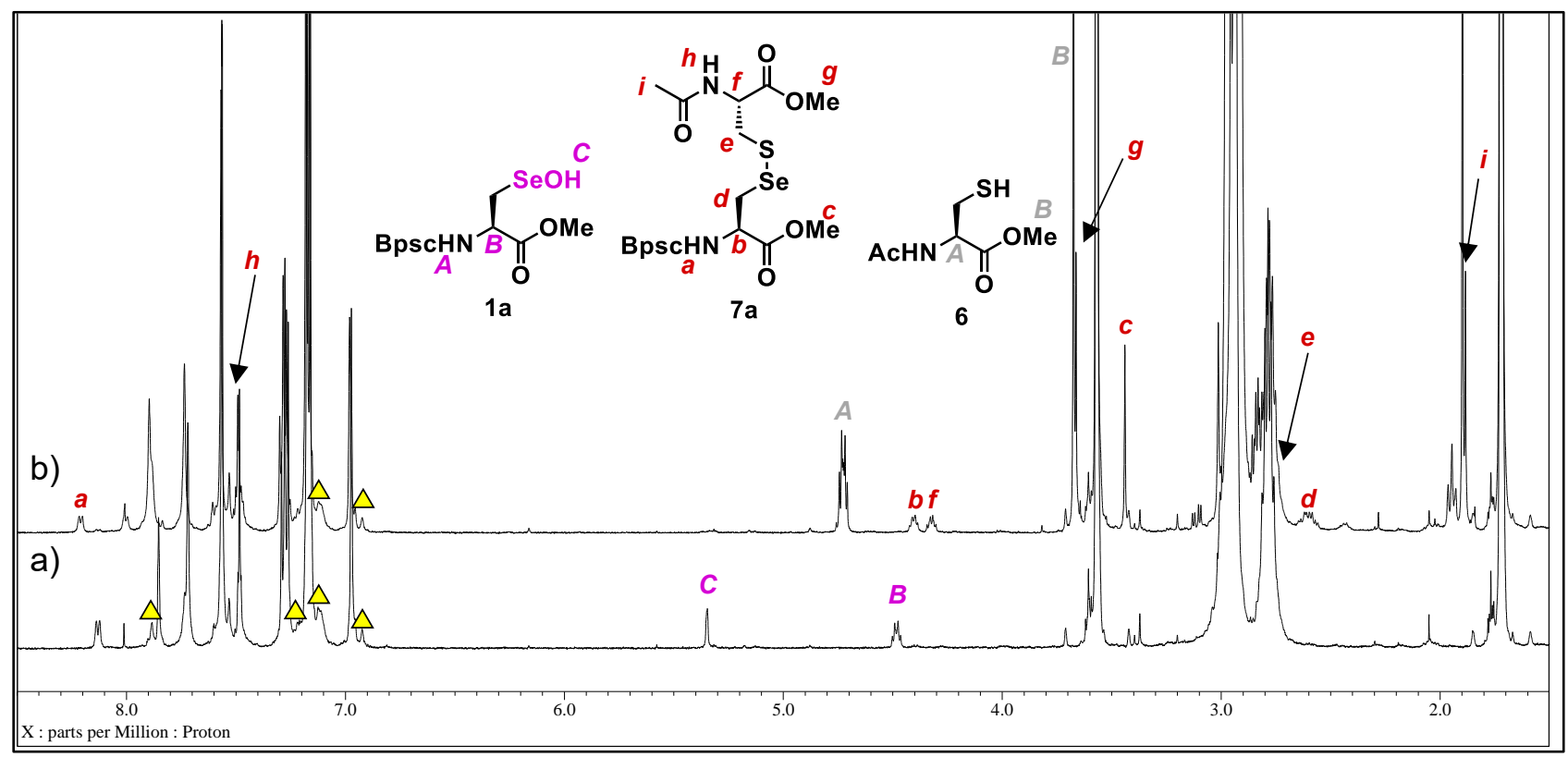

Figure S6. ${ }^{1} \mathrm{H}$ NMR $\left(500 \mathrm{MHz}, \mathrm{THF}-d_{8}\right)$ spectra of (a) before and (b) after addition of 6 in Scheme S21.

The obtained mixture was concentrated in vacuo and the crude product was purified by PTLC (hexane/ $\mathrm{CH}_{2} \mathrm{Cl}_{2}=$ 1:8) to give $\mathbf{7 a}$ as colorless crystals.

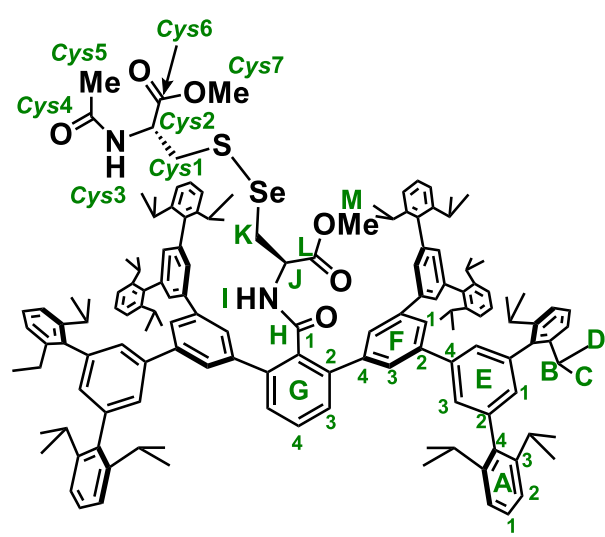

7a: colorless crystals; m.p. 203.0-205.2 ${ }^{\circ} \mathrm{C} .{ }^{1} \mathbf{H}$ NMR $(500 \mathrm{MHz}$, $\left.\mathrm{CDCl}_{3}\right): \delta 1.02-1.04(\mathrm{~m}, 48 \mathrm{H}, \mathbf{C}$ and $\mathbf{D}), 1.09-1.12(\mathrm{~m}, 48 \mathrm{H}, \mathbf{C}$ and $\mathbf{D})$, 1.86 (s, 3H, Cys5), $2.32\left(\mathrm{dd}, J=13.5,5.4 \mathrm{~Hz}, 1 \mathrm{H}, \mathbf{K}_{\mathrm{A}}\right), 2.68-2.79(\mathrm{~m}$, 17H, B and Cys $_{\mathbf{A}}$ ), $2.95\left(\mathrm{dd}, J=13.3,3.0 \mathrm{~Hz}, 1 \mathrm{H}, \mathbf{K}_{\mathbf{B}}\right), 3.00(\mathrm{~s}, 3 \mathrm{H}$, M), 3.09 (dd, $J=13.8,5.2 \mathrm{~Hz}, 1 \mathrm{H}$, Cys 1 $\left._{\mathbf{B}}\right), 3.52$ (s, 3H, Cys7), 4.49 (dt, $J=7.8,4.8 \mathrm{~Hz}, 1 \mathrm{H}$, Cys2), 4.70-4.73 (m, 1H, J), $6.28(\mathrm{~d}, J=7.5 \mathrm{~Hz}$, 1H, Cys3), 6.47 (d, $J=8.3 \mathrm{~Hz}, 1 \mathrm{H}, \mathbf{I}), 6.98$ (br, 4H, E1), 7.16-7.18 (m, 16H, A2), 7.31 (t, $J=7.7 \mathrm{~Hz}, 8 \mathrm{H}, \mathbf{A 1}), 7.41$ (d, $J=1.5 \mathrm{~Hz}, 8 \mathrm{H}, \mathbf{E 3})$, 7.42-7.44 (m, 2H, G3), 7.47-7.50 (m, 1H, G4), $7.66(\mathrm{~d}, J=1.4 \mathrm{~Hz}, 4 \mathrm{H}$, F3), 7.76 (br, 2H, F1); ${ }^{13} \mathbf{C ~ N M R ~ ( 1 2 5 ~ M H z , ~} \mathrm{CDCl}_{3}$ ): $\delta 22.9$ (q, Cys5), 24.3, 24.5 (q, C and D), 30.60, 30.62 (d, B), 32.3 (t, K), 40.9 (t, Cys1), 50.3 (d, J), 52.1 (d, Cys2), 52.4 (q, M, overlapped, q, Cys7), 122.7 (d, A2), 126.0 (d, F1), 126.6 (d, E3), 127.2 (d, F3), 128.1 (d, A1), 129.5 (d, G4), 130.0 (d, G3), 130.5 (d, E1), 134.7 (s, G1), 139.1 (s, A4), 140.1 (s, G2), 140.5 (s, F2), 141.2 (s, E2), 141.6 (s, F4), 141.8 (s, E4), 146.8 (s, A3), 167.9 (s, H), 169.9 (s, Cys4), 
170.3 (s, Cys6), 170.5 (s, L); ${ }^{77}$ Se NMR (95 MHz, CDCl $)$ ): $\delta$ 384; IR (KBr); 3423, 3326, 3059, 3032, 2961, 2926, 2867, 1747, 1675, 1578, 1498, 1460, 1437, 1382, 1361, 1345, 1325, 1250, 1215, 1178, 1103, 1055, 1039, 873, 827, $805,754,725 \mathrm{~cm}^{-1}$; HRMS (FD-TOF) $\mathrm{m} / z 2199.2259[\mathrm{M}]^{+}$(calcd for $\mathrm{C}_{149} \mathrm{H}_{174} \mathrm{~N}_{2} \mathrm{O}_{6} \mathrm{SSe}, 2199.2257$ ).

\section{Direct observation of Sec-SeOH $1 b$ by ${ }^{1} \mathrm{H}$ NMR spectroscopy.}

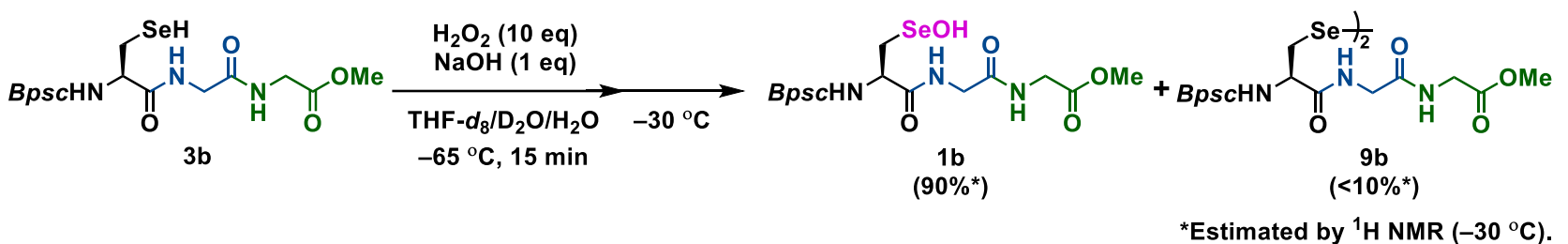

Scheme S22. Direct observation of Sec-SeOH 1 b.

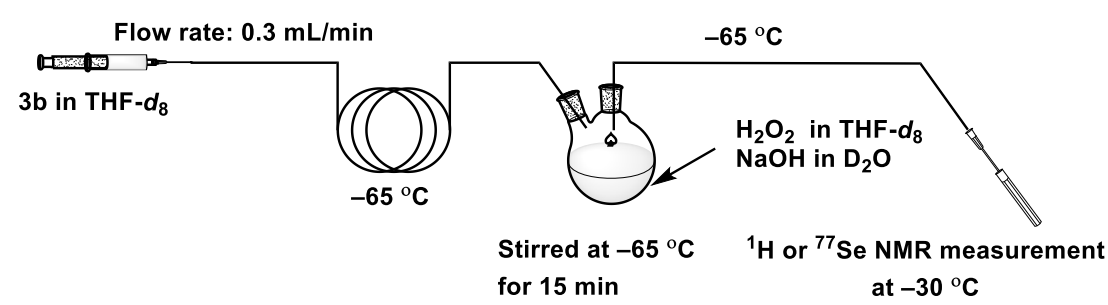

Figure S7. Protocol for direct observation of $\mathbf{1 b}$.

All solvents were degassed by argon bubbling before use. A stock solution of $\mathrm{H}_{2} \mathrm{O}_{2}(30 \%, 40 \mu \mathrm{L}, 0.39 \mathrm{mmol})$ in THF- $d_{8}(0.25 \mathrm{~mL})$ and that of $\mathrm{NaOH}(93 \%$ purity, $92 \mathrm{mg}, 2.1 \mathrm{mmol})$ in $\mathrm{D}_{2} \mathrm{O}(1.0 \mathrm{~mL})$ were prepared prior to the reaction. After evacuated and backfilled with argon, THF- $d_{8}(0.4 \mathrm{~mL})$, a stock solution of $\mathrm{NaOH}(2.1 \mathrm{M}, 3.0 \mu \mathrm{L}$, $6.3 \mu \mathrm{mol})$, and then that of $\mathrm{H}_{2} \mathrm{O}_{2}(1.3 \mathrm{M}, 87 \mu \mathrm{L}, 117 \mu \mathrm{mol})$ were added and the obtained mixture was cooled to $-65{ }^{\circ} \mathrm{C}$. After the flask was set in the system shown in Figure S7, selenol $3 \mathbf{b}(26.1 \mathrm{mg}, 12.2 \mu \mathrm{mol})$ and $1 \mu \mathrm{L}$ of bis(trimethylsilyl)methane as an internal standard in THF- $d_{8}(0.4 \mathrm{~mL})$ was added dropwise via a cooled tube $\left(-65^{\circ} \mathrm{C}\right)$ to the solution prepared as described above by using a syringe pump (flow rate: $0.3 \mathrm{~mL} / \mathrm{min}$ ). The obtained solution was stirred at $-65^{\circ} \mathrm{C}$ for $15 \mathrm{~min}$ and then transferred to a J-young NMR tube via a cooled tube $\left(-65^{\circ} \mathrm{C}\right)$ carefully. A ${ }^{1} \mathrm{H}$ NMR spectrum was recorded at $-30^{\circ} \mathrm{C}$ (Figure S8). Sec-SeOH $\mathbf{1 b}$ and the corresponding symmetric diselenide $\mathbf{9 b}$ were observed in $90 \%$ and $<10 \%$ yields, respectively and several signals were characterized (Figure S9). The signals of $\mathbf{9 b}$ in the ${ }^{1} \mathrm{H}$ NMR spectrum were in agreement with the compound data obtained in Scheme S23. 


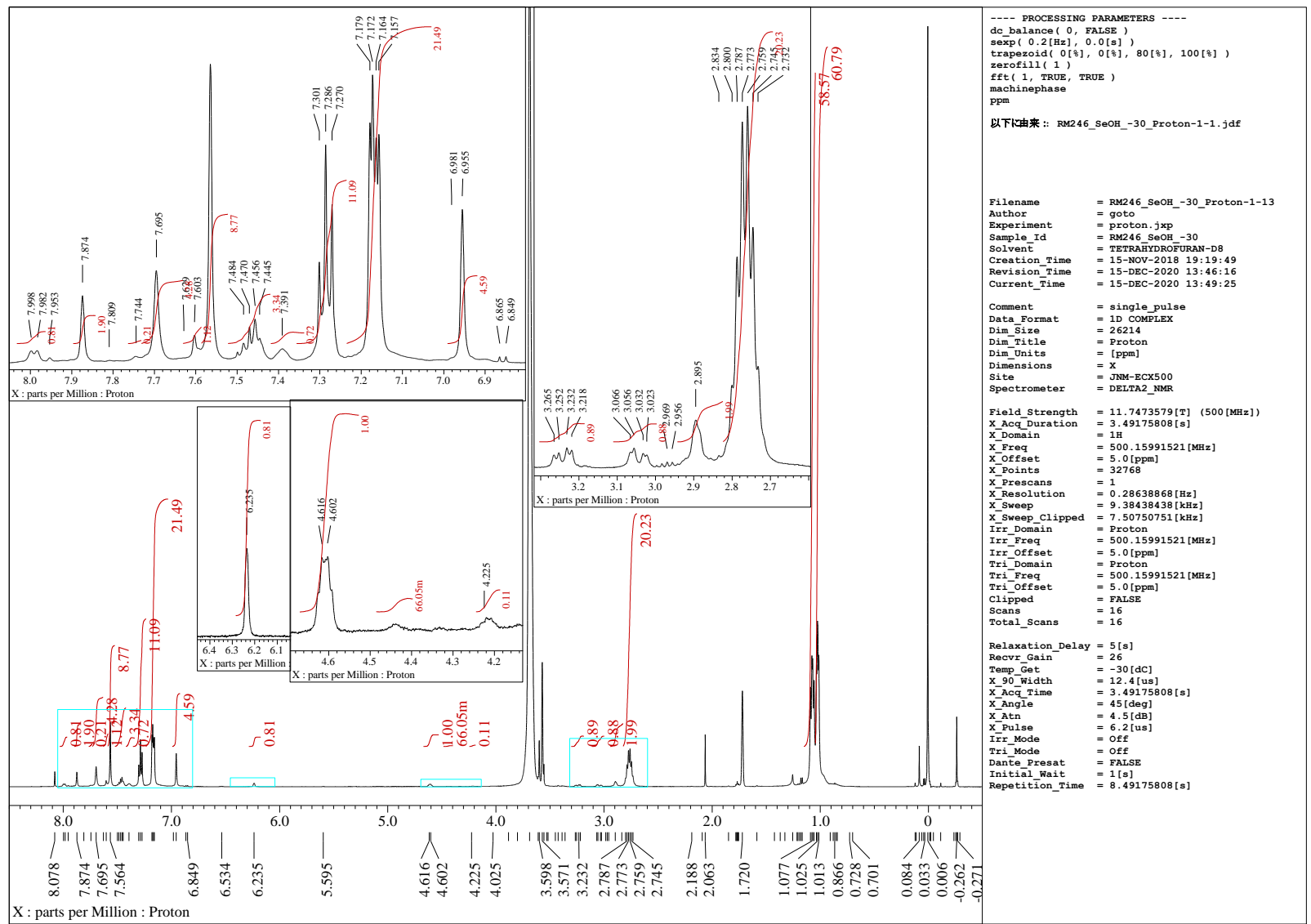

Figure S8. ${ }^{1} \mathrm{H}$ NMR $\left(500 \mathrm{MHz}, \mathrm{THF}-d_{8},-30{ }^{\circ} \mathrm{C}\right)$ spectrum of the mixture obtained in Scheme S22.

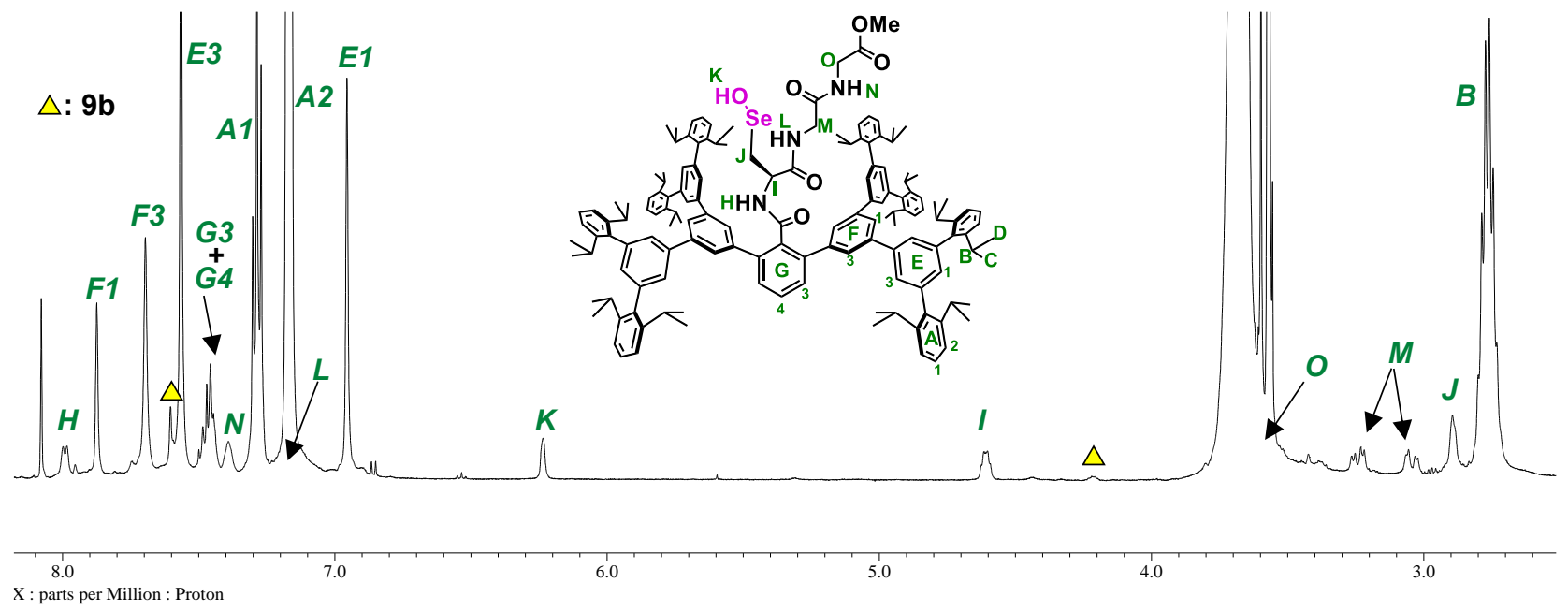

Figure S9. Assignment of the signal in the ${ }^{1} \mathrm{H}$ NMR $\left(500 \mathrm{MHz}\right.$, THF- $\left.d_{8},-30{ }^{\circ} \mathrm{C}\right)$ spectrum of the mixture obtained in Scheme S22. 
Direct observation of Sec-SeOH $1 \mathrm{~b}$ by ${ }^{77} \mathrm{Se}$ NMR spectroscopy.

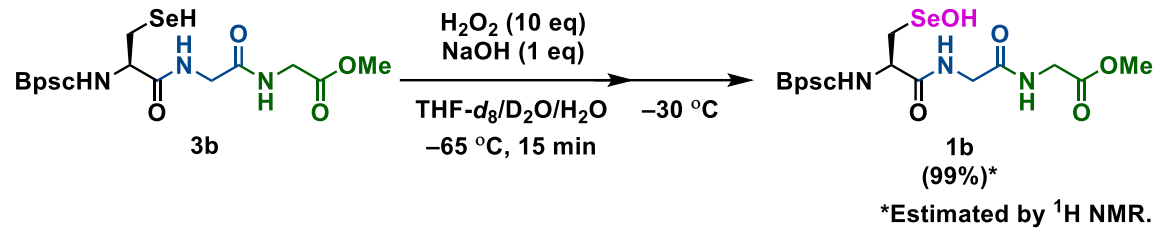

Scheme S23. Direct observation of $\mathrm{Sec}-\mathrm{SeOH} 1 \mathbf{b}$ by ${ }^{77} \mathrm{Se}$ NMR spectroscopy.

All solvents were degassed by argon bubbling before use. A stock solution of $\mathrm{NaOH}$ (93\% purity, $315 \mathrm{mg}, 7.32$ $\mathrm{mmol})$ in $\mathrm{D}_{2} \mathrm{O}(0.5 \mathrm{~mL})$ was prepared prior to the reaction. After evacuated and backfilled with argon, THF- $d_{8}(0.4$ $\mathrm{mL})$, a stock solution of $\mathrm{NaOH}(14.6 \mathrm{M}, 3.0 \mu \mathrm{L}, 44 \mu \mathrm{mol})$ and then $\mathrm{H}_{2} \mathrm{O}_{2}(30 \%, 41 \mu \mathrm{L}, 0.40 \mathrm{mmol})$ were added and the obtained mixture was cooled to $-65^{\circ} \mathrm{C}$. After the flask was set in the system shown in Figure S7, selenol 3b (87.3 mg, $40.8 \mu \mathrm{mol})$ in THF- $d_{8}(0.4 \mathrm{~mL})$ was added dropwise via a cooled tube $\left(-65^{\circ} \mathrm{C}\right)$ to the solution prepared as described above by using a syringe pump (flow rate: $0.07 \mathrm{~mL} / \mathrm{min}$ ). The obtained mixture was stirred at $-65^{\circ} \mathrm{C}$ for $15 \mathrm{~min}$ and then transferred to a J-young NMR tube via a cooled tube $\left(-65^{\circ} \mathrm{C}\right)$ carefully. The formation of Sec$\mathrm{SeOH} \mathbf{1 b}$ in a quantitative yield was observed by ${ }^{1} \mathrm{H}$ NMR spectrum at $-30{ }^{\circ} \mathrm{C}$ and then, a ${ }^{77} \mathrm{Se}$ NMR spectrum was recorded at $-30{ }^{\circ} \mathrm{C}$ (Figure S10). The obtained mixture was warmed to room temperature and transferred to a separatory funnel. After addition of water, the two layers were separated, and the aqueous layer was extracted with $\mathrm{CHCl}_{3}(3 \times 4 \mathrm{~mL})$. The combined organic layer was washed with brine, dried over $\mathrm{Na}_{2} \mathrm{SO}_{4}$, and filtered. The filtrate was concentrated in vacuo. After purification by $\mathrm{GPC}\left(\mathrm{CHCl}_{3}\right)$, dehydroalanine $\mathbf{8 b}$ was obtained as colorless crystals. Yield $61.1 \mathrm{mg}(29.7 \mu \mathrm{mol}, 73 \%)$.

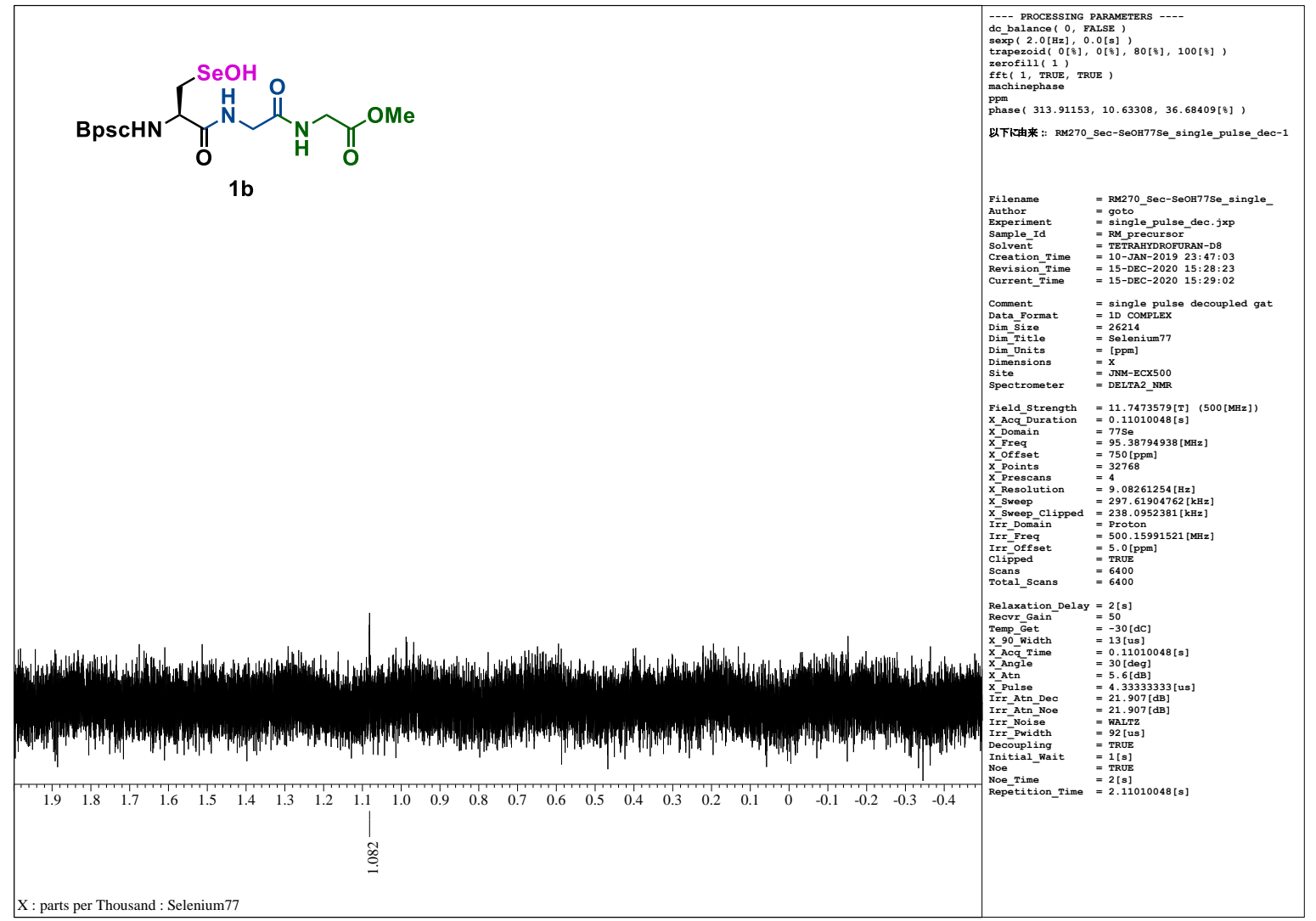

Figure S10. ${ }^{77} \mathrm{Se}$ NMR $\left(95 \mathrm{MHz}, \mathrm{THF}-d_{8},-30{ }^{\circ} \mathrm{C}\right)$ spectrum of the mixture obtained in Scheme $\mathrm{S} 23$. 


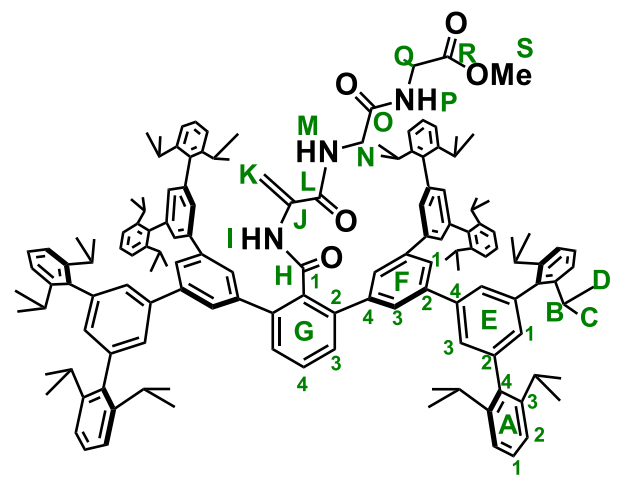

8b: colorless crystals; m.p. 222.9-225.3 ${ }^{\circ} \mathrm{C} .{ }^{1} \mathbf{H}$ NMR $(500 \mathrm{MHz}$, $\left.\mathrm{CDCl}_{3}\right): \delta 1.02(\mathrm{~d}, J=6.8 \mathrm{~Hz}, 48 \mathrm{H}, \mathbf{C}$ and $\mathbf{D}), 1.09(\mathrm{~d}, J=6.8 \mathrm{~Hz}, 48 \mathrm{H}$, C and D), 2.72 (septet, $J=6.8 \mathrm{~Hz}, 16 \mathrm{H}, \mathbf{B}), 3.39(\mathrm{~d}, J=4.6 \mathrm{~Hz}, 2 \mathrm{H}, \mathbf{N}$ ), $3.73(\mathrm{~s}, 3 \mathrm{H}, \mathbf{S}), 3.95(\mathrm{~d}, J=5.2 \mathrm{~Hz}, 2 \mathrm{H}, \mathbf{Q}), 4.34\left(\mathrm{~s}, 1 \mathrm{H}, \mathbf{K}_{\mathbf{A}}\right), 5.84(\mathrm{t}, J$ $=4.6 \mathrm{~Hz}, 1 \mathrm{H}, \mathbf{M}), 5.90(\mathrm{t}, J=5.2 \mathrm{~Hz}, 1 \mathrm{H}, \mathbf{P}), 6.09\left(\mathrm{~s}, 1 \mathrm{H}, \mathbf{K}_{\mathbf{B}}\right), 6.95(\mathrm{br}$, 4H, E1), 7.16 (d, $J=7.7 \mathrm{~Hz}, 16 \mathrm{H}, \mathbf{A 2}), 7.30$ (t, $J=7.7 \mathrm{~Hz}, 8 \mathrm{H}, \mathbf{A 1})$, 7.39 (br, 8H, E3), 7.52 (br, 3H, G3 and G4), 7.68 (br, 4H, F3), 7.77 (br, 2H, F1), 8.14 (s, 1H, I); ${ }^{13} \mathbf{C}$ NMR (125 MHz, CDCl $)$ ): $\delta 24.3,24.4$ (q, C and D), 30.5 (d, B), 41.2 (t, Q), 42.8 (t, N), 52.7 (q, S), 101.2 (t, K), 122.6 (d, A2), 126.0 (d, F1), 126.7 (d, E3), 127.0 (d, F3), 128.0 (d, A1), 129.6 (d, G3), 129.9 (d, G4), 130.2 (d, E1), 133.3 (s, J), 135.6 (s, G1), 139.3 (s, A4), 140.0 (s, G2), 140.7 (s, F2), 141.0 (s, E2, overlapped, s, F4), 141.9 (s, E4), 146.9 (s, A3), 162.7 (s, L), 167.9 (s, O), 168.2 (s, H), 169.9 (s, R); IR (KBr); 3409, 3365, 3060, 3033, 2961, 2926, 2867, 1751, 1698, 1665, 1631, 1578, 1486, 1464, 1400, 1383, 1362, 1346, 1325, 1250, 1207, 1179, 1102, 1055, 1039, 873, 805, 754, 725, 649, 631, 594 $\mathrm{cm}^{-1}$; HRMS (FD-TOF) $\mathrm{m} / 22056.3111[\mathrm{M}]^{+}$(calcd for $\left.\mathrm{C}_{147} \mathrm{H}_{169} \mathrm{~N}_{3} \mathrm{O}_{5}, 2056.3062\right)$.

Table S4. Comparison of the chemical shifts of $\mathbf{1 a}$ and $\mathbf{1 b}$ in ${ }^{77} \mathrm{Se}$ NMR spectra with those of previously reported selenenic acids.

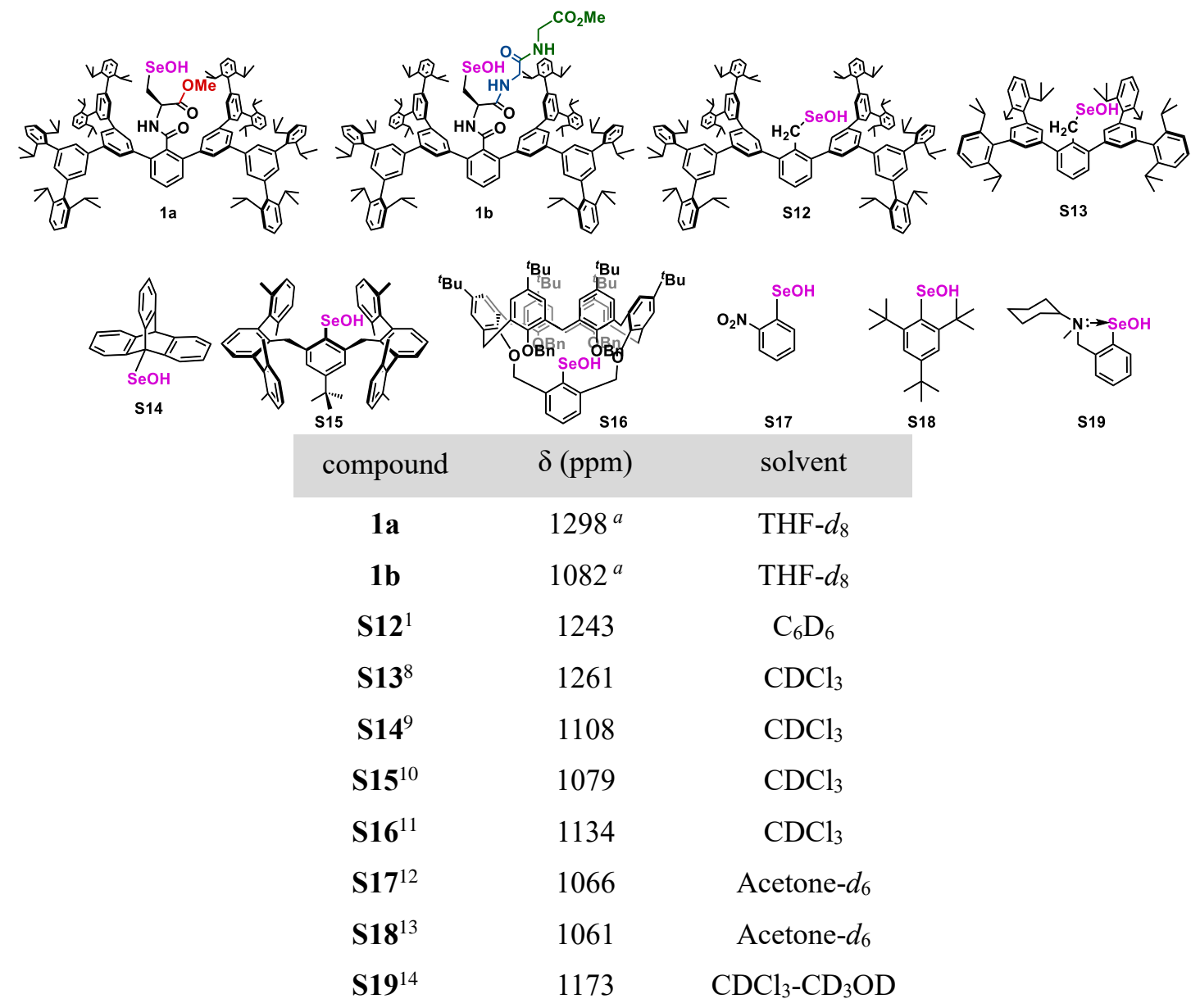

${ }^{a}$ Recorded at $-20{ }^{\circ} \mathrm{C}$. 
Derivatization of Sec-SeOH $1 \mathrm{~b}$ with acetylacetone (11).

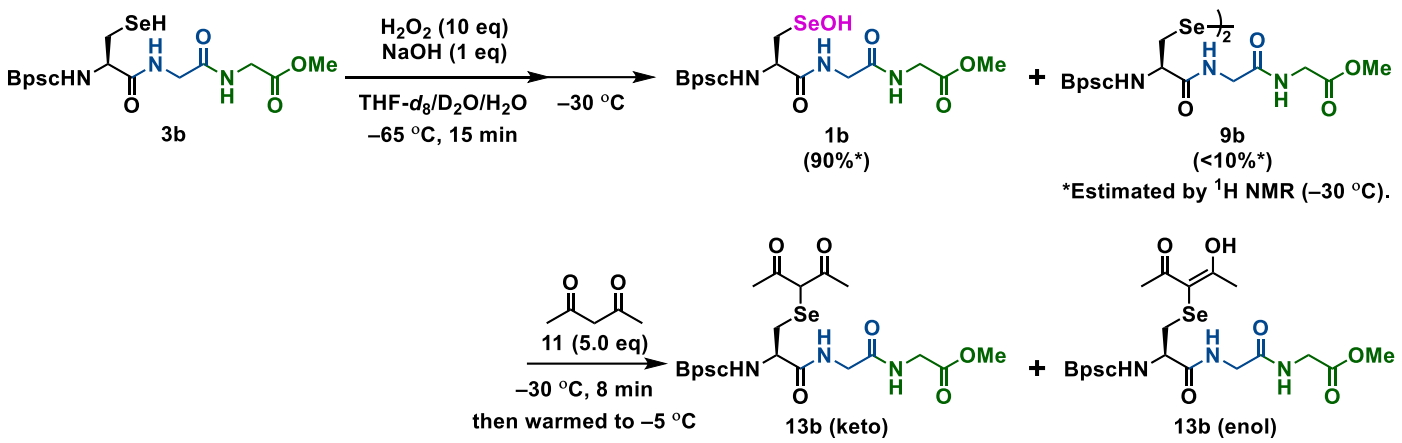

Scheme S24. Direct observation of $\mathbf{1 b}$ and its reaction with acetylacetone (11).

The mixture which was obtained in Scheme S22 was used for this experiment. After recording the ${ }^{1} \mathrm{H}$ NMR spectrum at $-30^{\circ} \mathrm{C}$, acetylacetone $(11 ; 6.3 \mu \mathrm{L}, 61 \mu \mathrm{mol}, 5.0 \mathrm{eq})$ was added at $-65^{\circ} \mathrm{C}$. The change of the components depending on the time was monitored by ${ }^{1} \mathrm{H}$ NMR spectroscopy at various temperature $\left(-30\right.$ to $-5{ }^{\circ} \mathrm{C}$; Table S5). The following workup was performed under argon atmosphere. After addition of degassed $10 \%$ aq. $\mathrm{Na}_{2} \mathrm{SO}_{3}(0.7$ $\mathrm{mL}$ ), the mixture was diluted with $\mathrm{Et}_{2} \mathrm{O}$. After the two layers were separated and the aqueous layer was extracted with $\mathrm{Et}_{2} \mathrm{O}(4 \times 2 \mathrm{~mL})$ using Schlenk tubes. The combined organic layer was dried over $\mathrm{Na}_{2} \mathrm{SO}_{4}$ and filtered through Celite using a glass filter. The filtrate was concentrated in vacuo. The composition of the crude mixture was determined by ${ }^{1} \mathrm{H}$ NMR spectrum. Selenide $\mathbf{1 3 b}$, symmetric diselenide $\mathbf{9 b}$, and dehydroalanine $\mathbf{8 b}$ were observed in 61\% (keto/enol $=38: 62$ ), 9\%, and 30\% NMR yields, respectively. The crude mixture was separated by PTLC $\left(\mathrm{CH}_{2} \mathrm{Cl}_{2}\right)$ to give selenide $\mathbf{1 3 b}$ (keto/enol $\left.=75: 25\right)$ as colorless crystals. The remained acetylacetone was removed by $\mathrm{GPC}\left(\mathrm{CHCl}_{3}\right)$. Yield $8.20 \mathrm{mg}(3.67 \mu \mathrm{mol}, 30 \%)$. Following data were collected for this mixture of tautomeric isomers of $\mathbf{1 3 b}$. Diselenide $\mathbf{9 b}$ was also obtained by the above PTLC separation as yellow crystals. Yield $1.0 \mathrm{mg}$ $(0.23 \mu \mathrm{mol}, 2 \%)$.

Table S5. Monitoring of the composition of the solution obtained in Scheme S24 after addition of $\mathbf{1 1}$ by ${ }^{1} \mathrm{H}$ NMR spectroscopy (500 MHz, THF- $\left.d_{8}\right)$.

\begin{tabular}{ccccccc} 
& & \multicolumn{6}{c}{ NMR yield (\%) } & \\
\cline { 3 - 6 } time (min) & temp. $\left({ }^{\circ} \mathrm{C}\right)$ & $\mathbf{1 b}$ & $\mathbf{8 b}$ & $\mathbf{9 b}$ & $\mathbf{1 3 b}$ & keto/enol ratio \\
8 & -30 & 48 & n.d. & 8 & 17 & $63: 37$ \\
20 & -20 & 15 & n.d. & 9 & 50 & $58: 42$ \\
29 & -5 & n.d. & n.d. & 13 & 63 & $62: 38$
\end{tabular}

13b $<$ keto/enol = 82:18>: colorless crystals; IR $(\mathrm{KBr})$; 3402, 3060, 3032, 2961, 2926, 2867, 1751, 1698, 1689, $1578,1508,1488,1466,1459,1416,1382,1362,1346,1179,1054,874,805,760,754,725,595 \mathrm{~cm}^{-1}$; HRMS (FD-TOF) $m / z 2236.2710[\mathrm{M}]^{+}$(calcd for $\mathrm{C}_{152} \mathrm{H}_{177} \mathrm{~N}_{3} \mathrm{O}_{7} \mathrm{Se}, 2236.2751$ ).

13b (keto form): ${ }^{1} \mathbf{H}$ NMR $\left(500 \mathrm{MHz}, \mathrm{CDCl}_{3}\right)$ : $\delta 1.00-1.10(\mathrm{~m}, 96 \mathrm{H}, \mathbf{C}$ and $\mathbf{D}), 1.55\left(\mathrm{dd}, J=13.0,4.1 \mathrm{~Hz}, 1 \mathrm{H}, \mathbf{K}_{\mathbf{A}}\right)$, $1.63(\mathrm{~s}, 3 \mathrm{H}, \mathbf{N}), 2.09$ (s, 3H, P), 2.67-2.79 (m, 17H, $\mathbf{B}$ and $\left.\mathbf{K}_{\mathbf{B}}\right), 2.98\left(\mathrm{dd}, J=17.0,4.2 \mathrm{~Hz}, 1 \mathrm{H}, \mathbf{S}_{\mathbf{A}}\right), 3.25(\mathrm{dd}, J=$ 17.6, $\left.5.3 \mathrm{~Hz}, 1 \mathrm{H}, \mathbf{V}_{\mathbf{A}}\right), 3.56(\mathrm{~s}, 3 \mathrm{H}, \mathbf{X}), 3.64\left(\mathrm{dd}, J=17.6,6.4 \mathrm{~Hz}, 1 \mathrm{H}, \mathbf{V}_{\mathbf{B}}\right), 3.73(\mathrm{~s}, 1 \mathrm{H}, \mathbf{L}), 3.93-3.98(\mathrm{~m}, 2 \mathrm{H}, \mathbf{J}$ and 


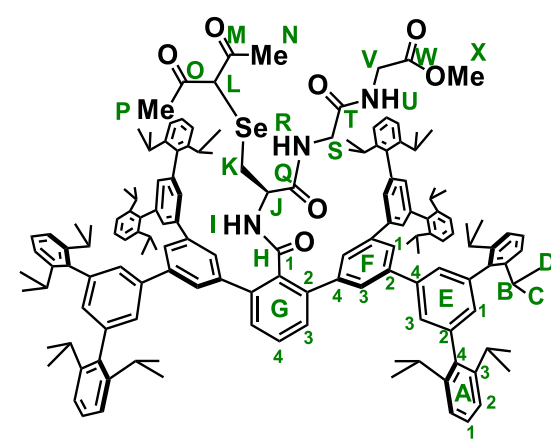

$\left.\mathbf{S}_{\mathbf{B}}\right), 6.39(\mathrm{dd}, J=8.8,4.2 \mathrm{~Hz}, 1 \mathrm{H}, \mathbf{R}), 6.77(\mathrm{t}, J=6.4 \mathrm{~Hz}, 1 \mathrm{H}, \mathbf{U}), 6.99(\mathrm{br}$, 4H, E1), 7.15-7.17 (m, 16H, A2), 7.30 (t, $J=7.8 \mathrm{~Hz}, 8 \mathrm{H}, \mathbf{A 1}), 7.47-7.48$ (m, 2H, G3), 7.48 (br, 8H, E3), 7.53-7.56 (m, 1H, G4), 7.66 (br, 4H, F3), 7.90 (br, $2 \mathrm{H}, \mathbf{F 1}), 8.72(\mathrm{~d}, J=4.3 \mathrm{~Hz}, 1 \mathrm{H}, \mathbf{I}) ;{ }^{13} \mathbf{C}$ NMR $\left(125 \mathrm{MHz}, \mathrm{CDCl}_{3}\right)$ : $\delta 22.4(\mathrm{t}, \mathbf{K}), 24.0,24.3,24.40,24.47,24.5$ (q, $\mathbf{C}$ and $\mathbf{D}), 28.2(\mathrm{q}, \mathbf{N}), 28.6$ (q, P), 30.5, 30.6 (d, B), $40.6(\mathrm{t}, \mathbf{V}), 42.3$ (t, S), 51.9 (q, X), 55.1 (d, J), 59.0 (s, L), 122.5, 122.71, 122.76 (d, A2), 125.9 (d, F1), 126.3 (d, E3), 126.8 (d, F3), 128.1 (d, A1), 129.6 (d, G4), 129.8 (d, G3), 130.7 (d, E1), 134.4 (s, G1), 139.0 (s, A4), 139.9 (s, F2), 140.2 (s, G2), 141.4 (s, E2), 141.6 (s, F4 overlapped with a carbon $\mathbf{F 4}$ in enol form, determined by HMBC), 141.7 (s, E4), 146.8, 146.9 (s, A3), 168.7 (s, T overlapped with a carbon $\mathbf{H}$ in enol form, determined by HMBC), 168.9 (s, Q), 169.3 (s, W), 172.0 (s, H), 199.2 (s, O), 201.0 (s, M); ${ }^{77} \mathbf{S e}$ NMR (95 MHz, CDCl 3 ): $\delta 203$ (br).

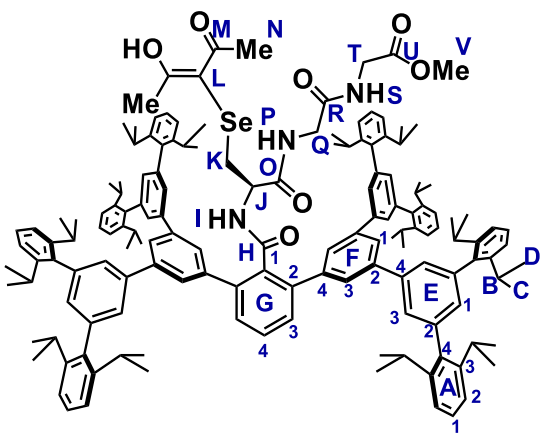

13b (enol form): ${ }^{1} \mathbf{H}$ NMR (500 MHz, $\left.\mathrm{CDCl}_{3}\right): \delta 1.02-1.12$ (m, 96H, $\mathbf{C}$ and D), $1.97(\mathrm{~s}, 6 \mathrm{H}, \mathbf{N}), 2.34\left(\mathrm{dd}, J=12.8,5.2 \mathrm{~Hz}, 1 \mathrm{H}, \mathbf{K}_{\mathrm{A}}\right), 2.44(\mathrm{dd}, J=13.0$, $\left.4.2 \mathrm{~Hz}, 1 \mathrm{H}, \mathbf{K}_{\mathbf{B}}\right), 2.57\left(\mathrm{dd}, J=17.2,3.7 \mathrm{~Hz}, 1 \mathrm{H}, \mathbf{Q}_{\mathbf{A}}\right), 2.67-2.79(\mathrm{~m}, 16 \mathrm{H}, \mathbf{B})$, $3.17\left(\mathrm{dd}, J=17.1,6.2 \mathrm{~Hz}, 1 \mathrm{H}, \mathbf{Q}_{\mathbf{B}}\right), 3.69$ (s, 3H, V), 3.70-3.76 (m, 1H, $\left.\mathbf{T}_{\mathbf{A}}\right)$, $3.86\left(\mathrm{dd}, J=18.2,5.6 \mathrm{~Hz}, 1 \mathrm{H}, \mathbf{T}_{\mathbf{B}}\right), 4.30(\mathrm{dt}, J=6.3,4.8 \mathrm{~Hz}, 1 \mathrm{H}, \mathbf{J}), 6.00-$ $6.04(\mathrm{~m}, 2 \mathrm{H}, \mathbf{P}$ and $\mathbf{S}), 6.51(\mathrm{~d}, J=6.6 \mathrm{~Hz}, 1 \mathrm{H}, \mathbf{I}), 6.98(\mathrm{t}, J=1.4 \mathrm{~Hz}, 4 \mathrm{H}$, E1), 7.15-7.19 (m, 16H, A2), 7.29-7.33 (m, 8H, A1), 7.42 (d, $J=1.4 \mathrm{~Hz}$, 8H, E3), 7.42-7.43 (m, 2H, G3), 7.48-7.52 (m, 1H, G4), 7.66 (br, 4H, F3), 7.81 (br, 2H, F1); ${ }^{13} \mathbf{C}$ NMR (125 MHz, $\mathrm{CDCl}_{3}$ ): $\delta$ 24.0, 24.2, 24.3, 24.40, 24.47, 24.5 (q, C and D), 25.8 (q, N), 28.9 (t, K), 30.5, 30.6 (d, B), 41.1 (t, T), 41.6 (t, Q), 51.8 (d, J), 52.4 (q, X), 97.9 (s, L), 122.5 (d, A2), 126.1 (d, F1), 126.5 (d, E3), 127.1 (d, F3), 128.1 (d, A1), 129.5 (d, G4), 130.2 (d, G3), 130.6 (d, E1), 134.7 (s, G1), 139.1 (s, A4), 140.1 (s, G2), 140.3 (s, F2), 141.2 (s, E2), 141.6 (s, F4 overlapped with a carbon F4 in keto form, determined by HMBC), 141.9 (s, E4), 146.9 (s, A3), 168.0 (s, R), 168.2 (s, O), 168.7 (s, H overlapped with a carbon T in keto form, determined by HMBC.), 169.8 (s, N), 196.7 (s, M); ${ }^{77} \mathbf{S e} \mathbf{N M R}\left(95 \mathrm{MHz}, \mathrm{CDCl}_{3}\right): \delta 150$.

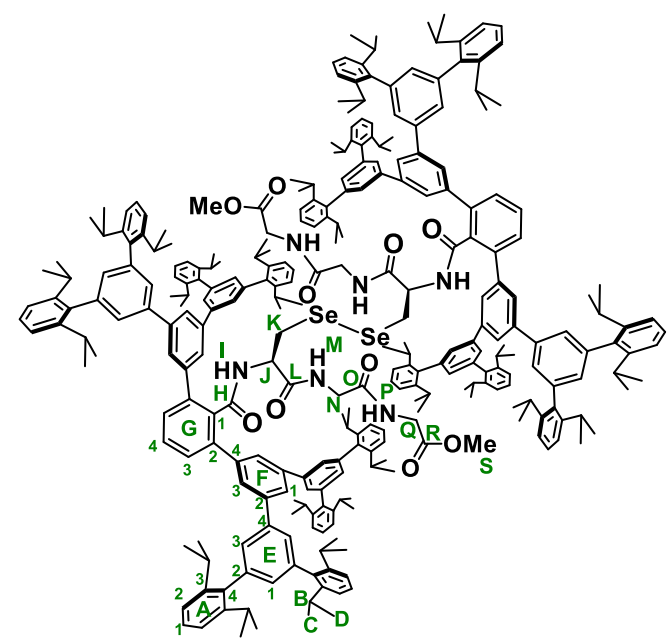

9b: pale yellow crystals; m.p. 244.6-246.1 ${ }^{\circ} \mathrm{C} .{ }^{1} \mathbf{H}$ NMR $(500 \mathrm{MHz}$, $\left.\mathrm{CDCl}_{3}\right): \delta$ 0.95-1.03 (m, 192H, $\mathbf{C}$ and $\left.\mathbf{D}\right), 2.21-2.25\left(\mathrm{~m}, 2 \mathrm{H}, \mathbf{K}_{\mathbf{A}}\right)$, 2.45-2.46 (m, 2H, K $\mathbf{B}), 2.65-2.72(\mathrm{~m}, 32 \mathrm{H}, \mathbf{B}), 2.93-2.97(\mathrm{~m}, 2 \mathrm{H}$, $\left.\mathbf{N}_{\mathbf{A}}\right), 3.17-3.20\left(\mathrm{~m}, 2 \mathrm{H}, \mathbf{N}_{\mathbf{B}}\right), 3.26(\mathrm{~s}, 6 \mathrm{H}, \mathbf{S}), 3.40-3.44\left(\mathrm{~m}, 2 \mathrm{H}, \mathbf{Q}_{\mathrm{A}}\right)$, $3.54\left(\mathrm{dd}, J=17.9,5.4 \mathrm{~Hz}, 2 \mathrm{H}, \mathbf{Q}_{\mathbf{B}}\right), 4.03-4.07$ (m, 2H, J), 6.32-6.39 (m, 6H, I, M, and P), 6.92 (br, 8H, E1), 7.07-7.12 (m, 32H, A2), 7.22 (t, $J=7.7 \mathrm{~Hz}, 16 \mathrm{H}, \mathbf{A 1}), 7.38-7.39$ (m, 4H, G3), 7.39 (br, 16H, E3), 7.43-7.46 (m, 2H, G4), 7.67 (br, 8H, F3), 7.81 (br, 4H, F1), ${ }^{13} \mathbf{C}$ NMR (125 MHz, $\left.\mathrm{CDCl}_{3}\right) \delta 24.2,24.3,24.52,24.54(\mathrm{q}, \mathbf{C}$ and D), 30.5 (d, B), $31.2(\mathrm{t}, \mathbf{K}), 40.8(\mathrm{t}, \mathbf{Q}), 42.8(\mathrm{t}, \mathbf{N}), 52.0(\mathrm{q}, \mathbf{S}), 53.2(\mathrm{~d}$, J), 122.6 (d, A2), 126.4 (d, F1), 126.8 (d, E3), 127.6 (d, F3), 128.1 (d, A1), 129.0 (d, G4), 130.5 (d, E1), 130.7 (d, G3), 134.3 (s, G1), 139.1 (s, A4), 140.1 (s, G2), 140.7 (s, F2), 141.2 (s, E2), 141.4 (s, F4), 141.9 (s, E4), 146.8, 146.9 (s, A3), 168.1 (s, L), 168.6 (s, H), 169.6 (s, O), 170.3 (s, R); ${ }^{77}$ Se 
NMR (95 MHz, CDCl $)$ ): not observed; IR (KBr); 3402 (br), 3060, 3032, 2961, 2926, 2867, 1750, 1683, 1578, 1507, 1486, 1464, 1382, 1362, 1325, 1250, 1207, 1179, 1054, 873, 805, 754, $725 \mathrm{~cm}^{-1}$; HRMS (ESI-TOF) $\mathrm{m} / \mathrm{z} 2161.2299$ $[\mathrm{M}+2 \mathrm{Na}]^{2+}$ (calcd for $\mathrm{C}_{294} \mathrm{H}_{340} \mathrm{~N}_{6} \mathrm{O}_{10} \mathrm{Se}_{2},[\mathrm{M}+2 \mathrm{Na}]^{2+}, 2161.2256$ ).

Model study on the bypass process (Step D) by utilizing Sec-SeOH $1 \mathrm{~b}$.

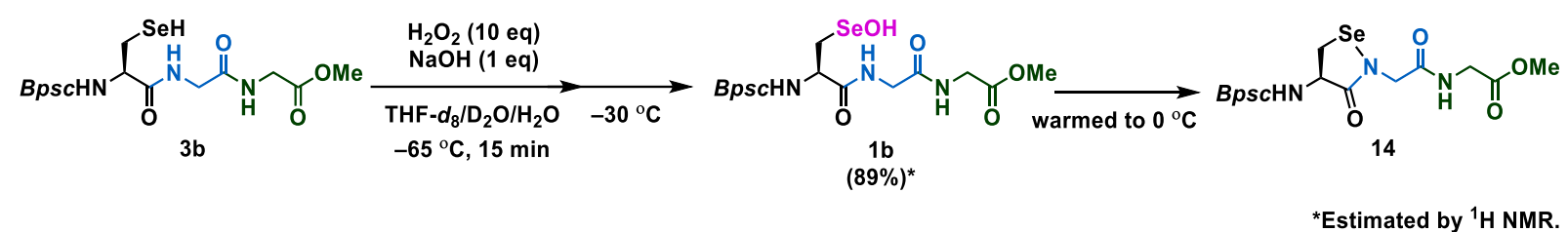

Scheme S25. Direct observation of Sec-SeOH $1 \mathbf{b}$ and its conversion to cyclic selenenyl amide 14.

All solvents were degassed by argon bubbling before use. A stock solution of $\mathrm{H}_{2} \mathrm{O}_{2}(30 \%, 40 \mu \mathrm{L}, 0.39 \mathrm{mmol})$ in THF- $d_{8}(0.25 \mathrm{~mL})$ and that of $\mathrm{NaOH}(93 \%$ purity, $113.6 \mathrm{mg}, 2.6 \mathrm{mmol})$ in $\mathrm{D}_{2} \mathrm{O}(1.0 \mathrm{~mL})$ were prepared prior to the reaction. After evacuated and backfilled with argon, THF- $d_{8}(0.4 \mathrm{~mL})$, a stock solution of $\mathrm{NaOH}(2.6 \mathrm{M}, 2.5 \mu \mathrm{L}$, $6.5 \mu \mathrm{mol})$ and then that of $\mathrm{H}_{2} \mathrm{O}_{2}(1.3 \mathrm{M}, 54 \mu \mathrm{L}, 73 \mu \mathrm{mol})$ were added and the obtained mixture was cooled to $-65^{\circ} \mathrm{C}$. After the flask was set in the system shown in Figure S7, selenol $\mathbf{3 b}(13.9 \mathrm{mg}, 6.50 \mu \mathrm{mol})$ and $1 \mu \mathrm{L}$ of bis(trimethylsilyl)methane as an internal standard in THF- $d_{8}(0.5 \mathrm{~mL})$ was added dropwise via a cooled tube $\left(-65^{\circ} \mathrm{C}\right)$ to the solution prepared as described above by using a syringe pump (flow rate: $0.3 \mathrm{~mL} / \mathrm{min}$ ). The obtained solution was stirred at $-65^{\circ} \mathrm{C}$ for $15 \mathrm{~min}$ and then transferred to a J-young NMR tube via a cooled tube $\left(-65^{\circ} \mathrm{C}\right)$ carefully. A ${ }^{1} \mathrm{H}$ NMR spectrum was measured at $-30{ }^{\circ} \mathrm{C}$. The mixture was warmed to $5{ }^{\circ} \mathrm{C}$ and ${ }^{1} \mathrm{H}$ NMR spectra at selected temperature were also recorded (Table S6, Figure S11). The corresponding cyclic selenenyl amide $\mathbf{1 4}$ was detected above $-10{ }^{\circ} \mathrm{C}$. The signals of 14 in the ${ }^{1} \mathrm{H}$ NMR spectrum were in agreement with those of the isolated sample prepared by another route (Scheme S26). Finally, the obtained mixture was allowed to stand at $-5{ }^{\circ} \mathrm{C}$ for 12 $\mathrm{h}$ and its composition was determined by ${ }^{1} \mathrm{H}$ NMR spectroscopy. Dehydroalanine $\mathbf{8 b}$ was observed in $74 \%$ yield.

Table S6. Monitoring of the composition of the solution obtained in Scheme S25 by ${ }^{1} \mathrm{H}$ NMR spectroscopies (400 MHz, THF- $\left.d_{8}\right)$.

\begin{tabular}{cccccc} 
& & \multicolumn{5}{c}{ NMR yield (\%) } \\
\cline { 3 - 6 } $\mathrm{t}(\mathrm{min})$ & temp. $\left({ }^{\circ} \mathrm{C}\right)$ & $\mathbf{1 b}$ & $\mathbf{1 4}$ & $\mathbf{8 b}$ & $\mathbf{9 b}$ \\
0 & -30 & 89 & n.d. & n.d. & 11 \\
6 & -20 & 87 & n.d. & n.d. & 11 \\
13 & -10 & 79 & 6 & n.d. & 11 \\
29 & 0 & 70 & 12 & 2 & 7 \\
35 & 0 & 67 & 15 & 5 & 5 \\
42 & 0 & 62 & 16 & 8 & 8 \\
52 & 0 & 53 & 15 & 9 & 8 \\
58 & 5 & 50 & 18 & 18 & 8 \\
$778^{a}$ & -5 & n.d. & n.d. & 74 & n.d.
\end{tabular}

${ }^{a}$ The mixture was allowed to stand at $-5^{\circ} \mathrm{C}$ for $12 \mathrm{~h}$. 


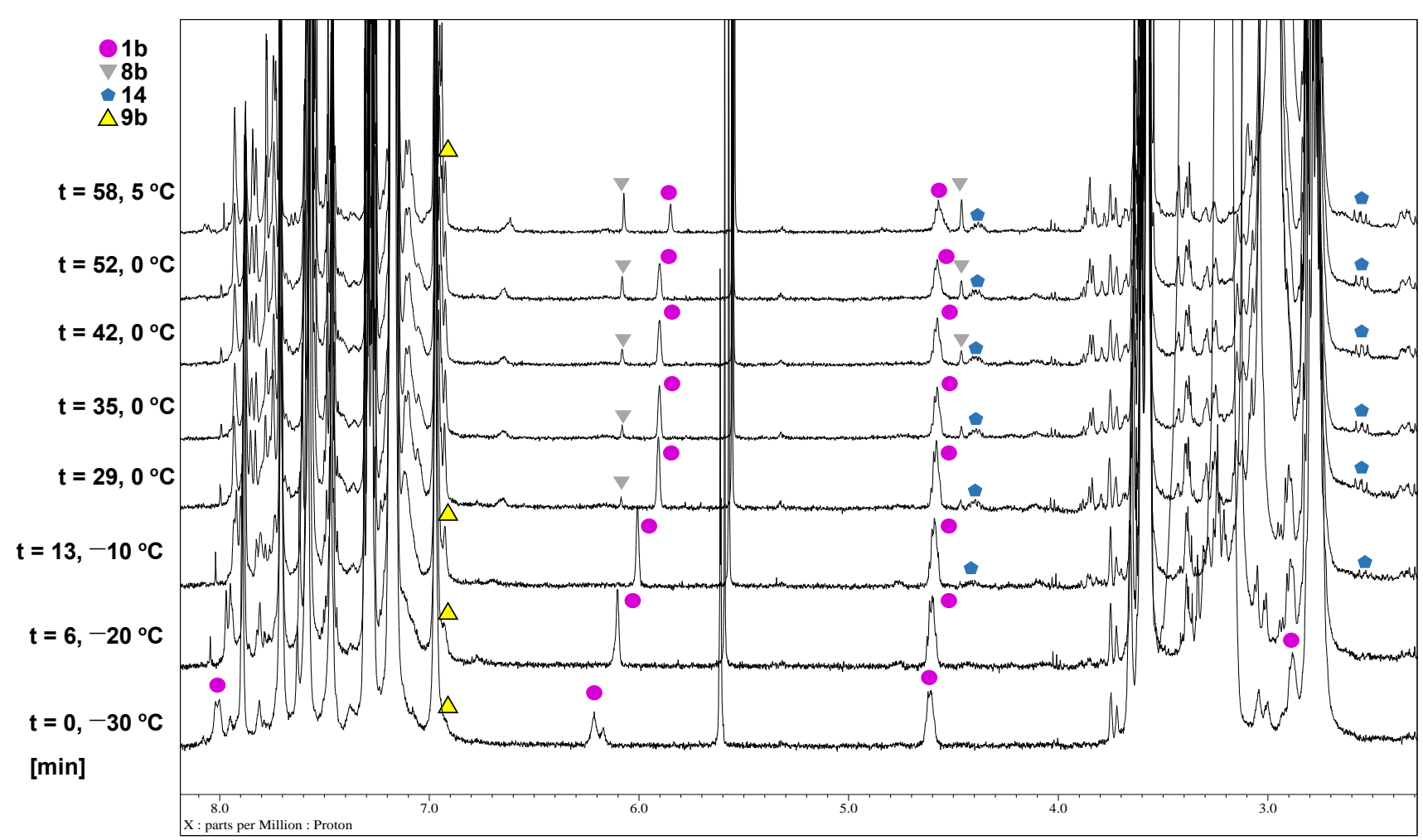

Figure S11. ${ }^{1} \mathrm{H}$ NMR $\left(400 \mathrm{MHz}, \mathrm{THF}-d_{8}\right)$ spectra of the solution obtained in Scheme S25.

Another synthetic route for cyclic selenenyl amide 14.

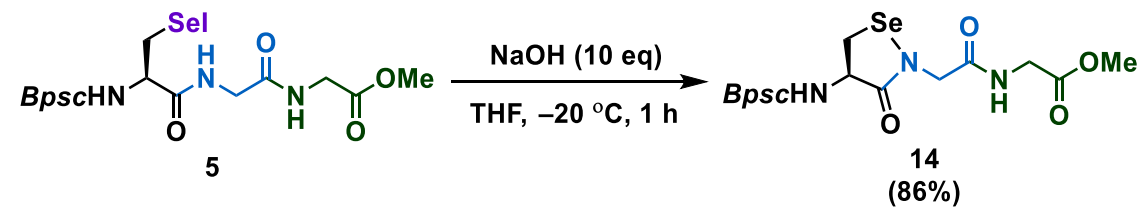

Scheme S26. Cyclization of selenenyl iodide 5 under basic conditions.

A stock solution of $\mathrm{NaOH}(93 \%$ purity, $187.7 \mathrm{mg}, 4.36 \mathrm{mmol})$ in degassed $\mathrm{H}_{2} \mathrm{O}(0.50 \mathrm{~mL})$ was prepared prior to the reaction. Selenenyl iodide $5(602.0 \mathrm{mg}, 0.2658 \mathrm{mmol})$ was placed in a $25 \mathrm{~mL}$ J-young tube. After evacuated and backfilled with argon, THF $(17 \mathrm{~mL})$ was added and the resulting brownish purple solution was cooled to $-20{ }^{\circ} \mathrm{C}$. The stock solution of $\mathrm{NaOH}(8.72 \mathrm{M}, 310 \mu \mathrm{L}, 2.71 \mathrm{mmol})$ was added and the resulting solution was stirred at the same temperature. After $1 \mathrm{~h}$, the color of the solution disappeared. The following workup was performed under argon atmosphere. Degassed $10 \%$ aq. $\mathrm{NH}_{4} \mathrm{Cl}(4.0 \mathrm{~mL})$ was added to the solution and then the layers were separated and the aqueous layer was extracted with $\mathrm{Et}_{2} \mathrm{O}(3 \times 4 \mathrm{~mL})$ using Schlenk tubes. The combined organic layer was dried over $\mathrm{Na}_{2} \mathrm{SO}_{4}$ and filtered through Celite using a glass filter. The filtrate was concentrated in vacuo and the crude product was purified by flash column chromatography on silica gel (hexane/ $\mathrm{CH}_{2} \mathrm{Cl}_{2}=2: 3$ ) to give $\mathbf{1 4}$ as pale-yellow crystals. Yield $486.8 \mathrm{mg}(0.2278 \mathrm{mmol}, 86 \%)$. 


\section{Characterization of selenenyl amide 14.}

Whether selenoamide $\mathbf{1 4}$ has a five-membered ring or an eight-membered ring was determined by HMBC measurement (Figure S12).

1. The protons $R$ of methoxy group in a terminal Gly residue correlated with the carbon $Q$ of amide-carbonyl group.

2. The carbon $Q$ correlated with the protons $P_{A}$ and $P_{B}$, both of which were observed as dd. These results are consistent with the existence of the amide proton $(O)$ adjacent to the protons $P_{A}$ and $P_{B}$. Actually, the protons $P_{A}$ and $P_{B}$ correlated with the amide proton $O$ through the amide-carbonyl carbon $Q$.

3. The proton $O$ correlated with not only the carbon $Q$ but also another carbon $N$.

4. The carbon $N$ correlated with the protons $M_{A}$ and $M_{B}$, both of which were observed as ABq. These results indicated that an amide proton adjacent to the protons $M_{A}$ and $M_{B}$ disappeared in the cyclization. It also suggests that the structure of $\mathbf{1 4}$ has a five-membered ring.

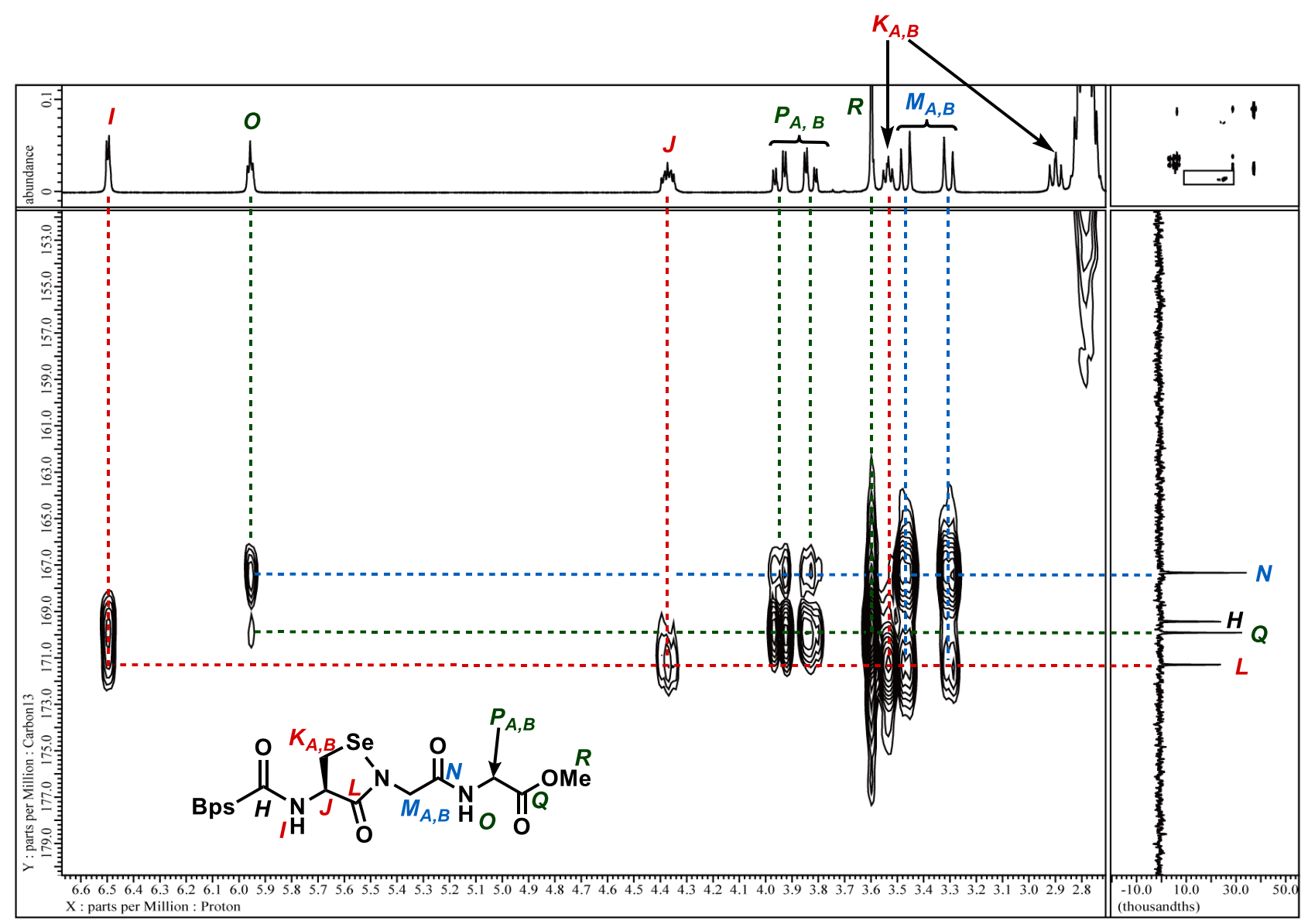

Figure S12. ${ }^{1} \mathrm{H}-{ }^{13} \mathrm{C}$ HMBC (X: $500 \mathrm{MHz}, \mathrm{Y}: 125 \mathrm{MHz}, \mathrm{CDCl}_{3}$ ) spectrum of selenenyl amide 14.

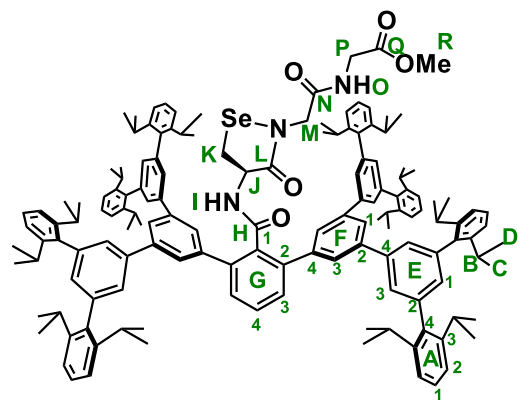

14: pale-yellow crystals; m.p. $212.0-214.9{ }^{\circ} \mathrm{C} .{ }^{1} \mathbf{H}$ NMR $\left(500 \mathrm{MHz}, \mathrm{CDCl}_{3}\right)$ $\delta$ 1.06-1.08 (m, 48H, C and D), $1.13(\mathrm{~d}, J=6.9 \mathrm{~Hz}, 24 \mathrm{H}, \mathrm{C}$ or D), $1.16(\mathrm{~d}, J$ $=6.9 \mathrm{~Hz}, 24 \mathrm{H}, \mathbf{C}$ or $\mathbf{D}), 2.72-2.83(\mathrm{~m}, 16 \mathrm{H}, \mathbf{B}), 2.89(\mathrm{dd}, J=11.6,9.9 \mathrm{~Hz}$, $\left.1 \mathrm{H}, \mathbf{K}_{\mathbf{A}}\right), 3.29\left(\mathrm{ABq}, J=16.6 \mathrm{~Hz}, 1 \mathrm{H}, \mathbf{M}_{\mathbf{A}}\right), 3.47\left(\mathrm{ABq}, J=16.6 \mathrm{~Hz}, 1 \mathrm{H}, \mathbf{M}_{\mathbf{B}}\right)$, $3.53\left(\mathrm{dd}, J=9.5,7.0 \mathrm{~Hz}, 1 \mathrm{H}, \mathbf{K}_{\mathbf{B}}\right), 3.59$ (s, $\left.3 \mathrm{H}, \mathbf{R}\right), 3.82(\mathrm{dd}, J=18.3,4.6 \mathrm{~Hz}$, $\left.1 \mathrm{H}, \mathbf{P}_{\mathbf{A}}\right), 3.94\left(\mathrm{dd}, J=18.4,5.2 \mathrm{~Hz}, 1 \mathrm{H}, \mathbf{P}_{\mathbf{B}}\right), 4.34-4.39(\mathrm{~m}, 1 \mathrm{H}, \mathbf{J}), 5.95(\mathrm{t}, J$ 
$=5.2 \mathrm{~Hz}, 1 \mathrm{H}, \mathbf{O}), 6.48(\mathrm{t}, J=4.9 \mathrm{~Hz}, 1 \mathrm{H}, \mathbf{I}), 7.02(\mathrm{br}, 4 \mathrm{H}, \mathbf{E 1}), 7.20-7.22(\mathrm{~m}, 16 \mathrm{H}, \mathbf{A 2}), 7.35(\mathrm{t}, J=7.8 \mathrm{~Hz}, 8 \mathrm{H}$, A1), 7.50 (d, $J=1.5 \mathrm{~Hz}, 8 \mathrm{H}, \mathbf{E 3}), 7.51-7.57$ (m, 3H, G3 and G4), 7.75 (d, $J=1.8 \mathrm{~Hz}, 4 \mathrm{H}, \mathbf{F 3}), 7.88$ (br, $2 \mathrm{H}, \mathbf{F 1})$;

${ }^{13}$ C NMR (125 MHz, $\left.\mathrm{CDCl}_{3}\right): \delta$ 24.3, $24.5(\mathrm{q}, \mathbf{C}$ and D), $29.6(\mathrm{t}, \mathbf{K}), 30.5,30.6(\mathrm{~d}, \mathbf{B}), 41.0(\mathrm{t}, \mathbf{P}), 48.0(\mathrm{t}, \mathbf{M}), 52.4$ (q, R), 54.3 (d, J), 122.68, 122.69 (d, A2), 126.0 (d, F1), 126.6 (d, E3), 127.0 (d, F3), 128.0 (d, A1), 129.6 (d, G4), 129.7 (d, G3), 130.4 (d, E1), 135.0 (s, G1), 139.2 (s, A4), 140.2 (s, G2), 140.5 (s, F2), 141.2 (s, E2), 141.5 (s, F4), 141.8 (s, E4), $146.91,146.95$ (s, A3), 167.4 (s, N), 169.5 (s, H), 169.9 (s, Q), 171.3 (s, L); ${ }^{77}$ Se NMR (95 MHz, $\mathrm{CDCl}_{3}$ ): $\delta$ 876; IR (KBr); 3394 (br), 3060, 3032, 2961, 2926, 2867, 1749, 1670, 1578, 1519, 1488, 1461, 1519, 1488, 1461, 1382, 1362, 1346, 1325, 1249, 1209, 1179, 1055, 873, 805, 754, $725 \mathrm{~cm}^{-1}$; HRMS (FD-TOF) $\mathrm{m} / \mathrm{z}$ 2136.2302 [M] $]^{+}$calcd for $\mathrm{C}_{148} \mathrm{H}_{171} \mathrm{~N}_{2} \mathrm{O}_{5} \mathrm{Se}, 2136.2227$ ).

Reaction of cyclic selenenyl amide 14 with cysteine thiol 6.
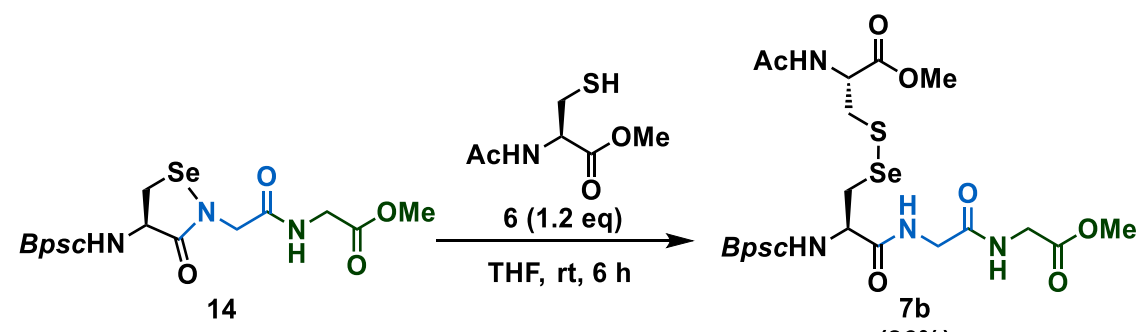

$(86 \%)$

Scheme S27. Reaction of cyclic selenenyl amide 14 with cysteine thiol 6.

Cyclic selenenyl amide $14(133.4 \mathrm{mg}, 62.43 \mu \mathrm{mol})$ was placed in a $25 \mathrm{~mL}$ Schlenk tube. After evacuated and backfilled with argon, THF $(8.5 \mathrm{~mL})$ and $N$-acetyl cysteine methyl ester $(6 ; 13.6 \mathrm{mg}, 76.7 \mathrm{mmol})$ were added. The resulting solution was stirred at room temperature for $6 \mathrm{~h}$ before water was added. The two layers were separated and the aqueous layer was extracted with $\mathrm{CHCl}_{3}(3 \times 4 \mathrm{~mL})$. The combined organic layer was washed with brine, dried over $\mathrm{Na}_{2} \mathrm{SO}_{4}$, and filtered. The filtrate was concentrated in vacuo and the crude product was purified by flash column chromatography on silica gel $\left(\mathrm{CH}_{2} \mathrm{Cl}_{2} / \mathrm{MeOH}=50: 1\right)$ to give $7 \mathbf{b}$ as colorless crystals. Yield $124.4 \mathrm{mg}(53.76$ $\mu \mathrm{mol}, 86 \%$ ). This reaction proceeded cleanly without any additives.

Direct reduction of cyclic selenenyl amide 14 to selenol $3 \mathrm{~b}$.

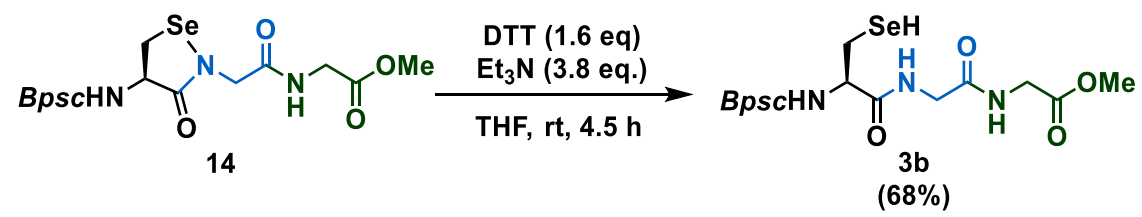

Scheme S28. Reduction of cyclic selenenyl amide 14 by DTT.

Cyclic selenenyl amide $14(115.0 \mathrm{mg}, 53.82 \mu \mathrm{mol})$ was placed in a $10 \mathrm{~mL}$ Schlenk tube. After evacuated and backfilled with argon, THF $(2.5 \mathrm{~mL})$ and $\mathrm{Et}_{3} \mathrm{~N}(28 \mu \mathrm{L}, 0.20 \mathrm{mmol})$ were added. The obtained solution was transferred to a solution of D, L-dithiothreitol $(13.4 \mathrm{mg}, 86.8 \mu \mathrm{mol})$ in THF $(5.0 \mathrm{~mL})$ in a separate $25 \mathrm{~mL}$ Schlenk tube. The resulting solution was stirred at room temperature for $4.5 \mathrm{~h}$. The following workup was performed under argon atmosphere. After addition of saturated aq. $\mathrm{NH}_{4} \mathrm{Cl}(3.0 \mathrm{~mL})$ and diluted by $\mathrm{CHCl}_{3}$ (freshly purified using $\mathrm{Al}_{2} \mathrm{O}_{3}$ and degassed), degassed brine was added to the solution and then the two layers were separated. The aqueous 
layer was extracted with $\mathrm{CHCl}_{3}(4 \times 2 \mathrm{~mL})$ using Schlenk tubes. The combined organic layer was dried over $\mathrm{Na}_{2} \mathrm{SO}_{4}$ and filtered using a glass filter. The filtrate was concentrated in vacuo and the crude product was immediately purified by column chromatography on silica gel (hexane/ $\mathrm{CH}_{2} \mathrm{Cl}_{2}=1: 8$ ) to give the $\mathbf{3 b}$ as colorless crystals. Yield $78.4 \mathrm{mg}(36.7 \mu \mathrm{mol}, 68 \%)$.

\section{Reaction of Sec-SeOH 1b with cysteine thiol 6.}

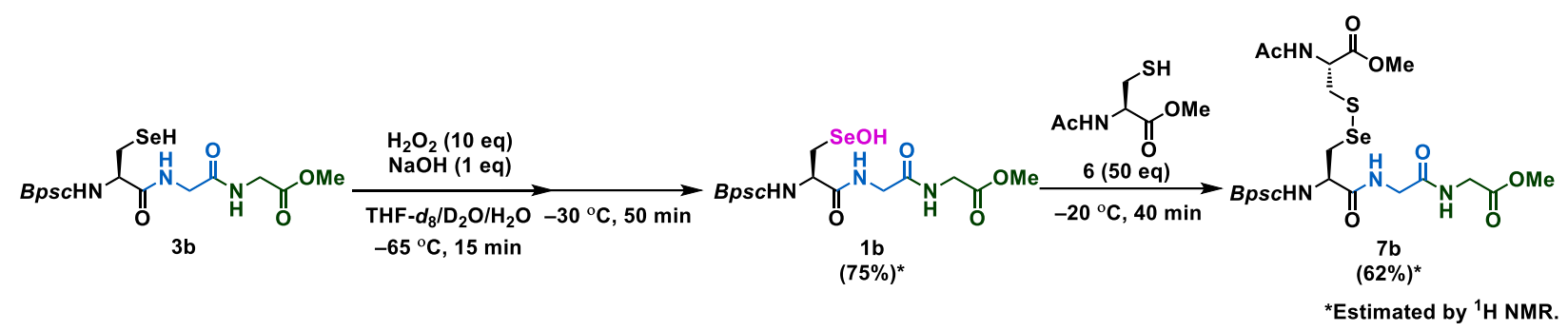

Scheme S29. Direct observation of the $\mathrm{Sec}-\mathrm{SeOH} \mathbf{1 b}$ and its reaction with cysteine thiol 6.

All solvents were degassed by argon bubbling before use. A stock solution of $\mathrm{H}_{2} \mathrm{O}_{2}(30 \%, 40 \mu \mathrm{L}, 0.39 \mathrm{mmol})$ in THF- $d_{8}(0.25 \mathrm{~mL})$ and that of $\mathrm{NaOH}(93 \%$ purity, $95.5 \mathrm{mg}, 2.2 \mathrm{mmol})$ in $\mathrm{D}_{2} \mathrm{O}(1.0 \mathrm{~mL})$ were prepared prior to the reaction. After evacuated and backfilled with argon, THF- $d_{8}(0.4 \mathrm{~mL})$, a stock solution of $\mathrm{NaOH}(2.2 \mathrm{M}, 3.3 \mu \mathrm{L}$, $7.3 \mu \mathrm{mol})$, and then that of $\mathrm{H}_{2} \mathrm{O}_{2}(1.3 \mathrm{M}, 60 \mu \mathrm{L}, 81 \mu \mathrm{mol})$ were added and the obtained mixture was cooled to $-65{ }^{\circ} \mathrm{C}$. After the flask was set in the system shown in Figure S7, selenol 3b (15.4 mg, $\left.7.20 \mu \mathrm{mol}\right)$ and $2 \mu \mathrm{L}$ of bis(trimethylsilyl)methane as an internal standard in THF- $d_{8}(0.50 \mathrm{~mL})$ was added dropwise via a cooled tube $\left(-65^{\circ} \mathrm{C}\right)$ to the solution prepared as described above by using a syringe pump (flow rate: $0.3 \mathrm{~mL} / \mathrm{min}$ ). The obtained solution was stirred at $-65^{\circ} \mathrm{C}$ for 15 min and then transferred to a J-young NMR tube via a cooled tube $\left(-65^{\circ} \mathrm{C}\right)$ carefully. The sample was allowed to stand at $-30{ }^{\circ} \mathrm{C}$ for $50 \mathrm{~min}$ for the VT-NMR measurement. The formation of Sec-SeOH $1 \mathbf{b}$ in $75 \%$ yield was observed by ${ }^{1} \mathrm{H}$ NMR measurement at $-30{ }^{\circ} \mathrm{C}$. Then, a solution of $N$-acetyl cysteine methyl ester $(\mathbf{6} ; 63.8 \mathrm{mg}, 0.360 \mathrm{mmol}, 50 \mathrm{eq})$ in THF- $d_{8}(0.2 \mathrm{~mL})$ was added to the above mixture at $-65^{\circ} \mathrm{C}$. After $40 \mathrm{~min}$, disappearance of $\mathrm{Sec}-\mathrm{SeOH} \mathbf{1} \mathbf{b}$ and formation of the corresponding selenenyl sulfide $\mathbf{7 b}$ were observed by ${ }^{1} \mathrm{H}$ NMR measurement at $-20{ }^{\circ} \mathrm{C}$. The signals of $\mathbf{7 b}$ in the ${ }^{1} \mathrm{H}$ NMR spectrum were in agreement with those of the isolated sample. The following workup was performed under argon atmosphere. After the obtained mixture in a Jyoung NMR tube was transferred to a $10 \mathrm{~mL}$ Schlenk tube and diluted with $\mathrm{Et}_{2} \mathrm{O}(4 \mathrm{~mL})$, degassed $10 \%$ aq. $\mathrm{Na}_{2} \mathrm{SO}_{3}$ $(1.0 \mathrm{~mL})$ and sat. aq. $\mathrm{NH}_{4} \mathrm{Cl}(1.0 \mathrm{~mL})$ were added to the solution. The two layers were separated and then the aqueous layer was extracted with $\mathrm{Et}_{2} \mathrm{O}(4 \times 2 \mathrm{~mL})$ using Schlenk tubes. The combined organic layer was dried over $\mathrm{Na}_{2} \mathrm{SO}_{4}$ and filtered using a glass filter. The filtrate was concentrated in vacuo and the crude product was immediately purified by PTLC $\left(\mathrm{MeOH} / \mathrm{CH}_{2} \mathrm{Cl}_{2}=1: 50\right)$ to give selenenyl sulfide $\mathbf{7 b}$ as colorless crystals. Yield 10.6 $\operatorname{mg}(4.58 \mu \mathrm{mol}, 59 \%)$. 
Oxidation of 14 with $\mathrm{H}_{2} \mathrm{O}_{2}$.

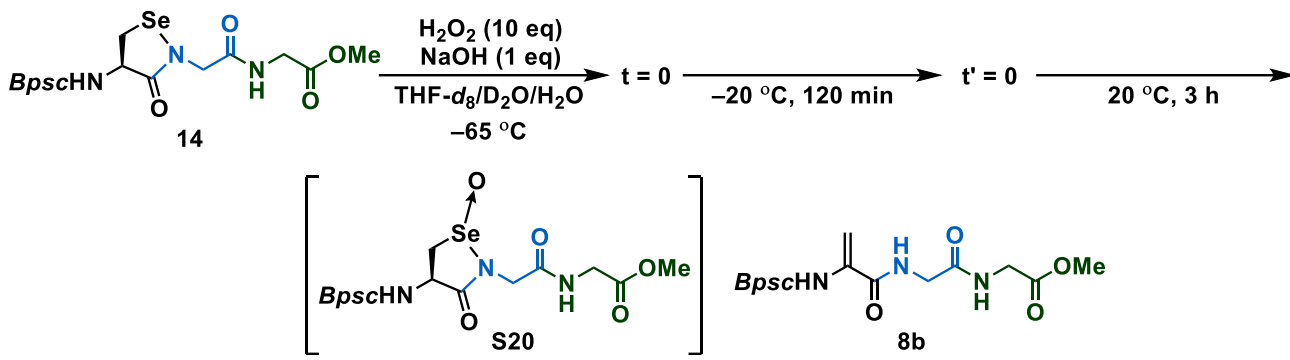

Scheme S30. Oxidation of 14 by $\mathrm{H}_{2} \mathrm{O}_{2}$.

All solvents were degassed by argon bubbling before use. A stock solution of $\mathrm{NaOH}$ (93\% purity, $99.0 \mathrm{mg}, 2.3$ $\mathrm{mmol})$ in $\mathrm{D}_{2} \mathrm{O}(1.0 \mathrm{~mL})$ was prepared prior to the reaction. Cyclic selenenyl amide $\mathbf{1 4}(19.6 \mathrm{mg}, 9.17 \mu \mathrm{mol})$ was placed in a J-Young NMR tube. After evacuated and backfilled with argon, THF- $d_{8}(0.70 \mathrm{~mL})$ was added at room temperature and then $\mathrm{H}_{2} \mathrm{O}_{2}(30 \%, 9.4 \mu \mathrm{L}, 92 \mu \mathrm{mol})$ and the stock solution of $\mathrm{NaOH}(2.3 \mathrm{M}, 4.0 \mu \mathrm{L}, 9.2 \mu \mathrm{mol})$ were added at $-65{ }^{\circ} \mathrm{C}$. The sample was allowed to stand at $-20{ }^{\circ} \mathrm{C}$ for $17 \mathrm{~min}$ for the VT-NMR measurement. Cyclic selenenyl amide $\mathbf{1 4}$ and an intermediate which is highly likely to be selenoxide $\mathbf{S 2 0}$ were observed in $89 \%$ and $7 \%$ yields, respectively, by ${ }^{1} \mathrm{H}$ NMR measurement at $-20{ }^{\circ} \mathrm{C}$. Subsequently, the ${ }^{1} \mathrm{H}$ NMR spectra at $-20{ }^{\circ} \mathrm{C}$ were also recorded over $2 \mathrm{~h}$ (Table S7 and Figure S13). Then, the reaction mixture was warmed to $20{ }^{\circ} \mathrm{C}$ and the ${ }^{1} \mathrm{H}$ NMR spectra at $20^{\circ} \mathrm{C}$ were also recorded over $3 \mathrm{~h}$ (Table S8 and Figure S14). Although the intermediate of the reaction was not isolated, the observed signals of the intermediate are consistent with the formation of the diastereomers of selenoxide S20.

Table S7. Monitoring of the composition of the solution obtained in Scheme S30 at $-20{ }^{\circ} \mathrm{C}$ by ${ }^{1} \mathrm{H}$ NMR spectroscopies $\left(500 \mathrm{MHz}, \mathrm{THF}-d_{8} / \mathrm{D}_{2} \mathrm{O}\right)$.

\begin{tabular}{ccccc} 
& & \multicolumn{4}{c}{ NMR yield (\%) } \\
\cline { 3 - 5 } $\mathrm{t}(\mathrm{min})$ & temp. $\left({ }^{\circ} \mathrm{C}\right)$ & $\mathbf{1 4}$ & $\mathbf{S 2 0}$ & $\mathbf{8 b}$ \\
17 & -20 & 89 & 7 & n.d. \\
36 & -20 & 88 & 10 & n.d. \\
57 & -20 & 87 & 11 & n.d. \\
78 & -20 & 86 & 11 & n.d. \\
99 & -20 & 85 & 11 & n.d. \\
114 & -20 & 86 & 10 & n.d.
\end{tabular}


Table S8. Monitoring of the composition of the solution obtained in Scheme S30 at $20{ }^{\circ} \mathrm{C}$ by ${ }^{1} \mathrm{H}$ NMR spectroscopies $\left(500 \mathrm{MHz}, \mathrm{THF}-d_{8} / \mathrm{D}_{2} \mathrm{O}\right)$.

\begin{tabular}{ccccc} 
& & \multicolumn{3}{c}{ NMR yield (\%) } \\
\cline { 3 - 5 } t' $($ min $)$ & temp. $\left({ }^{\circ} \mathrm{C}\right)$ & $\mathbf{1 4}$ & $\mathbf{S 2 0}$ & $\mathbf{8 b}$ \\
6 & 20 & 78 & n.d. & 10 \\
17 & 20 & 66 & 6 & 14 \\
27 & 20 & 46 & 23 & 18 \\
37 & 20 & 22 & 41 & 24 \\
48 & 20 & 9 & 58 & 36 \\
56 & 20 & trace & 56 & 41 \\
100 & 20 & n.d. & 30 & 59 \\
183 & 20 & n.d. & 10 & 81
\end{tabular}

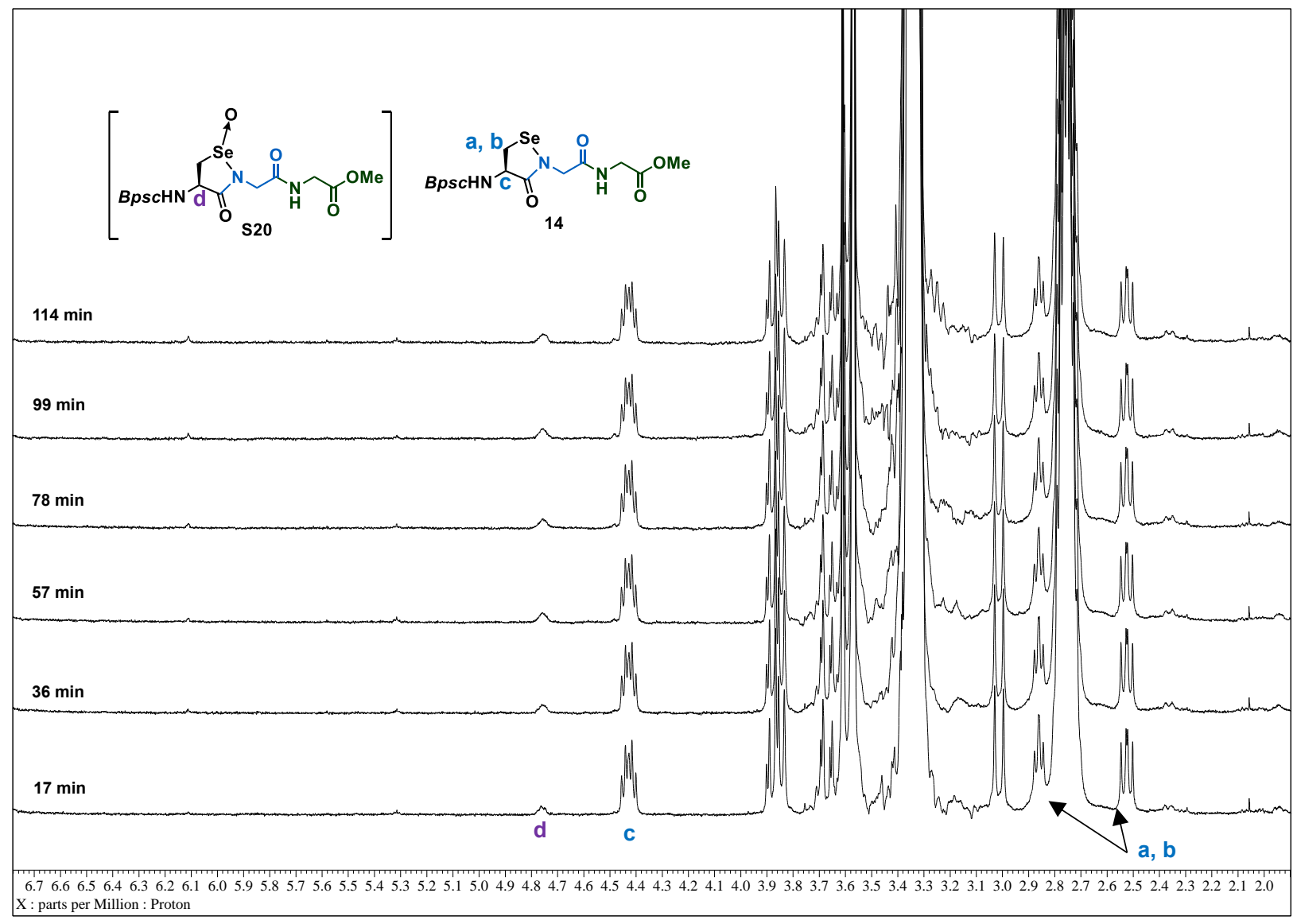

Figure S13. ${ }^{1} \mathrm{H}$ NMR $\left(500 \mathrm{MHz}, \mathrm{THF}-d_{8} / \mathrm{D}_{2} \mathrm{O}\right)$ monitoring of the reaction shown in Scheme $\mathrm{S} 30$ at $-20{ }^{\circ} \mathrm{C}$. 


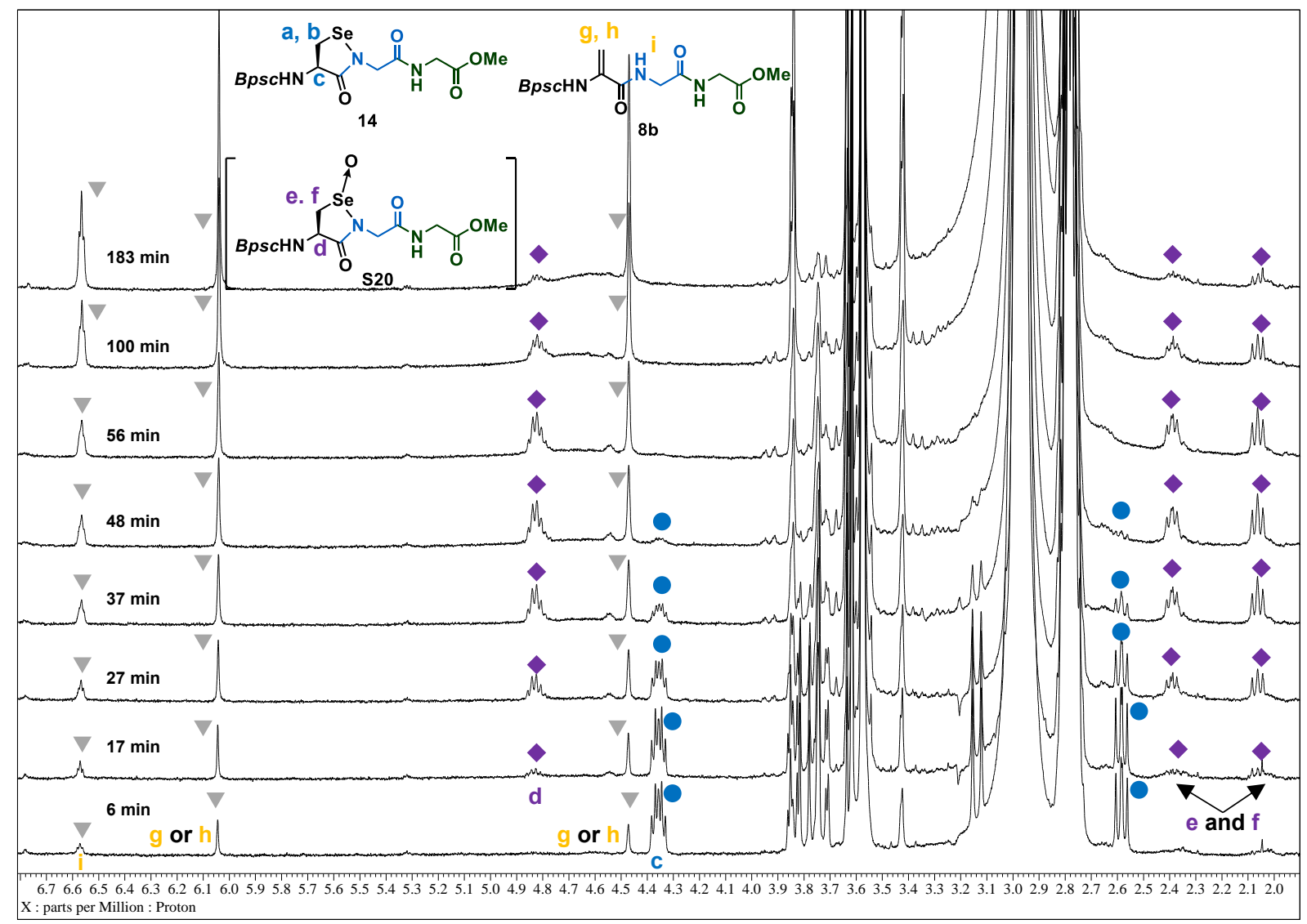

Figure S14. ${ }^{1} \mathrm{H}$ NMR $\left(500 \mathrm{MHz}\right.$, THF- $\left.d_{8} / \mathrm{D}_{2} \mathrm{O}\right)$ monitoring of the reaction shown in Scheme $\mathrm{S} 30$ at $20{ }^{\circ} \mathrm{C}$.

\section{X-ray crystallographic analysis of selenenyl iodide 5.}

Single crystals of $2(5) \cdot 1.5 \mathrm{C}_{4} \mathrm{H}_{10} \mathrm{O} \cdot 1.5 \mathrm{C}_{5} \mathrm{H}_{12}$ were grown in their $\mathrm{Et}_{2} \mathrm{O}$-pentane solution. A brownish crystal of 2(5) $1.5 \mathrm{C}_{4} \mathrm{H}_{10} \mathrm{O} \cdot 1.5 \mathrm{C}_{5} \mathrm{H}_{12}$ was mounted on a loop. All measurements were made on a Rigaku/Synergy CCD with VariMax $\mathrm{Cu}$ with graphite monochromated $\mathrm{Cu}-\mathrm{K} \alpha$ radiation $(\lambda=1.54184 \AA)$ at $-150{ }^{\circ} \mathrm{C}$. Crystallographic and experimental data are listed in Table S9. The structures were solved and refined against all $F_{2}$ values using Shelx2018 implemented through Olex2 v1.3. The non-hydrogen atoms were refined anisotropically, except for the solvent molecules, the minor components of the disordered isopropyl groups, and an amide carbonyl carbon on a peptide moiety of the central glycine residue of one of the two independent molecules. The hydrogen atoms were idealized by using the riding models. Attempt to sensibly model the solvent molecules (probably one and half pentane which was used for final crystallization) was unsuccessful because of diffuse electron density (disordered) corresponding to them and limited data quality. So, the solvent mask (similar to PLATON_SQUEEZE) was applied using Olex2 to remove those electron densities in the final model. The solvent accessible volume was found to be $757.5 \AA^{3}$. The number of the electrons found in solvent accessible void is $79.2 \mathrm{e}$, which corresponds to approximately two pentane molecules per unit cell. The slight variation in void electron count can be the result of limited data quality. 
Table S9. Crystal data and structure refinement for $2(\mathbf{5}) \cdot 1.5 \mathrm{C}_{4} \mathrm{H}_{10} \mathrm{O} \bullet 1.5 \mathrm{C}_{5} \mathrm{H}_{12}$.

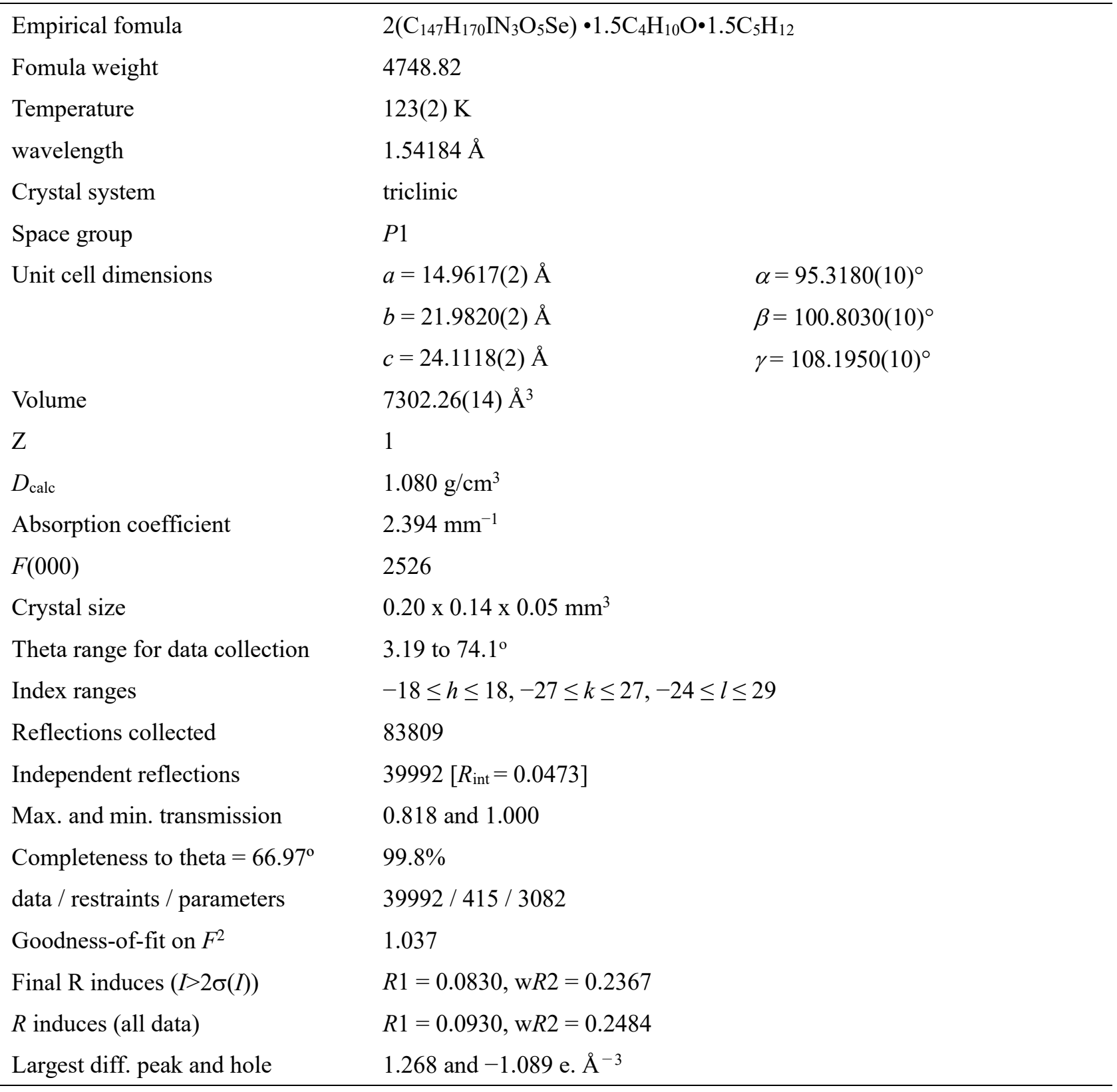




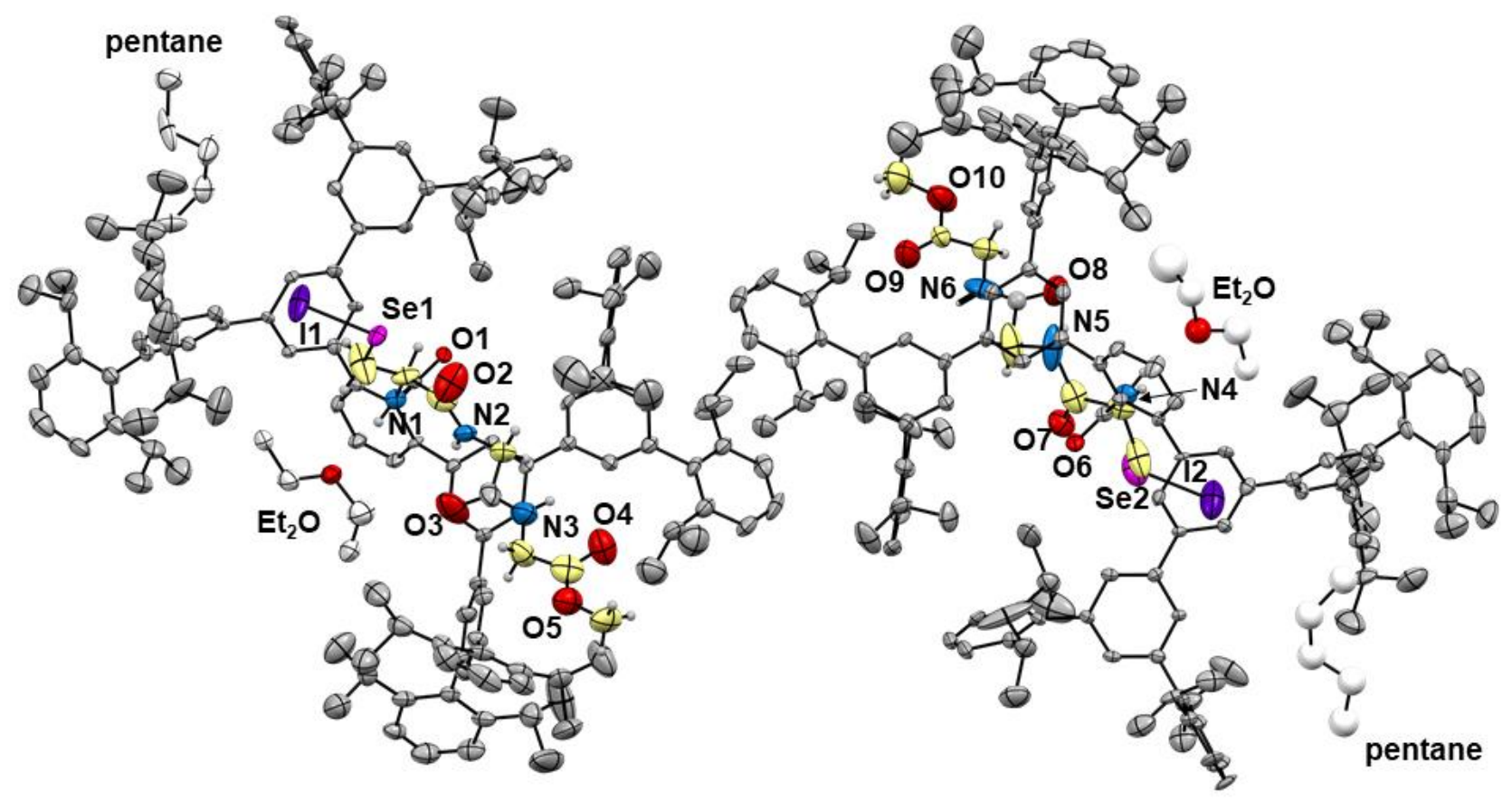

Figure S15. Molecular structure of 5 with thermal ellipsoids at 50\% probability (CCDC 2063205). Only the position with higher occupancy of the disordered isopropyl group in the Bpsc group and the disordered Se-I bonds in the selenocysteine moiety are shown. Hydrogen atoms of the Bpsc groups and solvents are omitted for clarity.
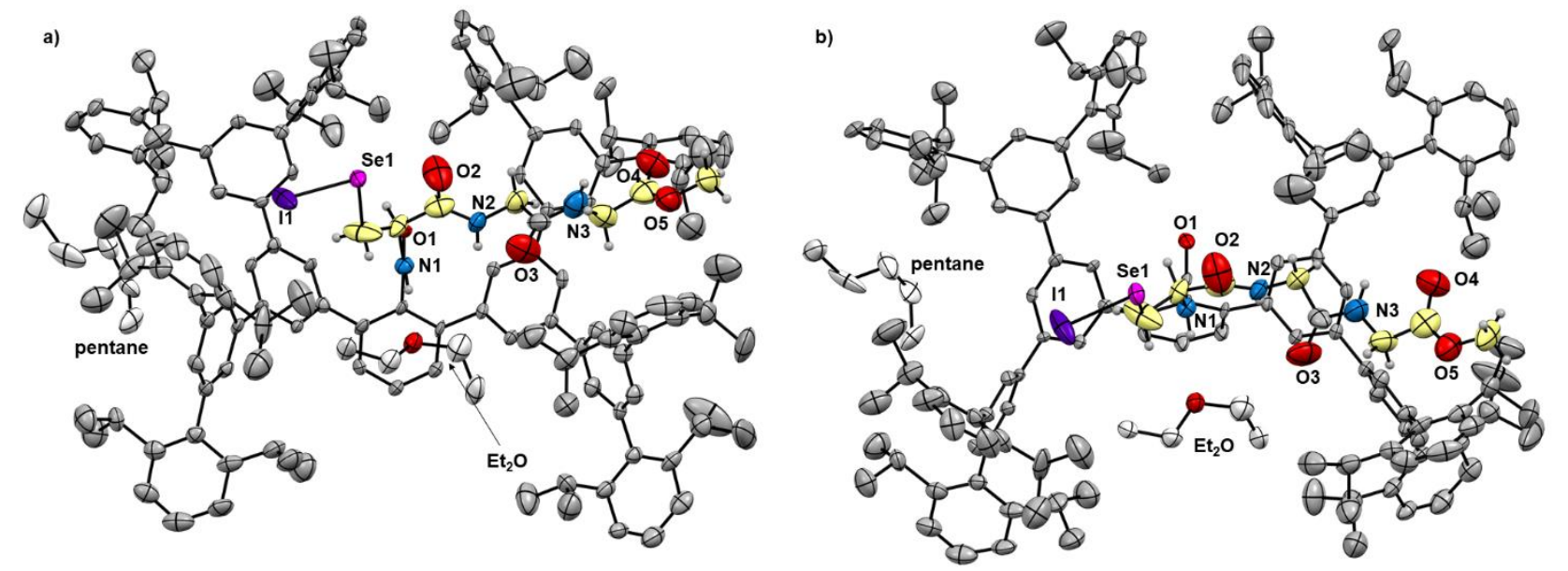

Figure S16. Selected molecular structure of 5 with thermal ellipsoids at 50\% probability (CCDC 2063205): (a) Front view, and (b) top view. Only the position with higher occupancy of the disordered isopropyl group in the Bpsc group and the disordered Se-I bonds in the selenocysteine moiety are shown. Hydrogen atoms of the Bpsc groups and solvents are omitted for clarity. 
Table S10. Selected structural parameters of selenenyl iodides 5, S21, and S22.

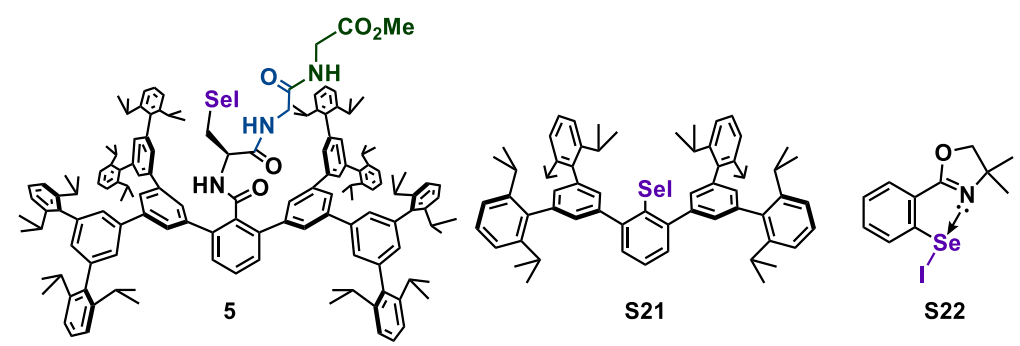

\begin{tabular}{ccccc} 
& \multicolumn{2}{c}{ bond length $(\AA)$} & & bond angle (deg) \\
\cline { 2 - 3 } compounds & Se-I & C-Se & C-Se-I \\
\cline { 2 - 3 } $\mathbf{5}$ (molecule A, major) & $2.5395(19)$ & $2.000(10)$ & $92.6(4)$ \\
$\mathbf{5}$ (molecule A, minor) & $2.537(4)$ & $1.935(10)$ & \\
$\mathbf{5}$ (molecule B, major) & $2.5013(16)$ & $1.960(8)$ & \\
$\mathbf{5}$ (molecule B, minor) & $2.769(5)$ & $1.960(8)$ & $97.3(3)$ \\
S21 $^{15}$ & $2.5203(11)$ & $1.946(5)$ & \\
S22 $^{16}$ & $2.7773(7)$ & $1.945(5)$ & & $102.14(15)$ \\
& & & $97.39(14)$
\end{tabular}


NMR spectra

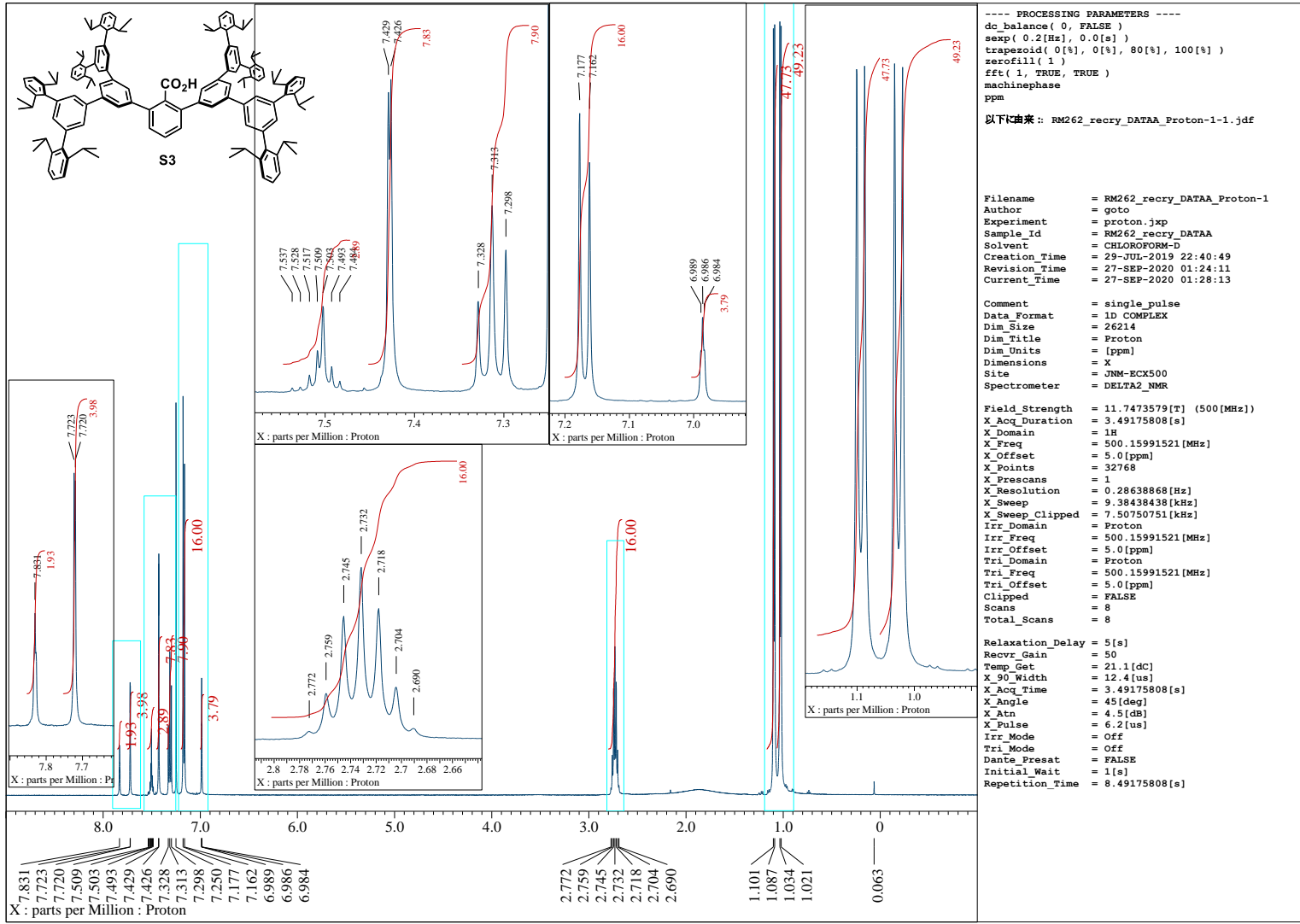

Figure S17. ${ }^{1} \mathrm{H}$ NMR $\left(500 \mathrm{MHz}, \mathrm{CDCl}_{3}\right)$ spectrum of $\mathbf{S 3}$.

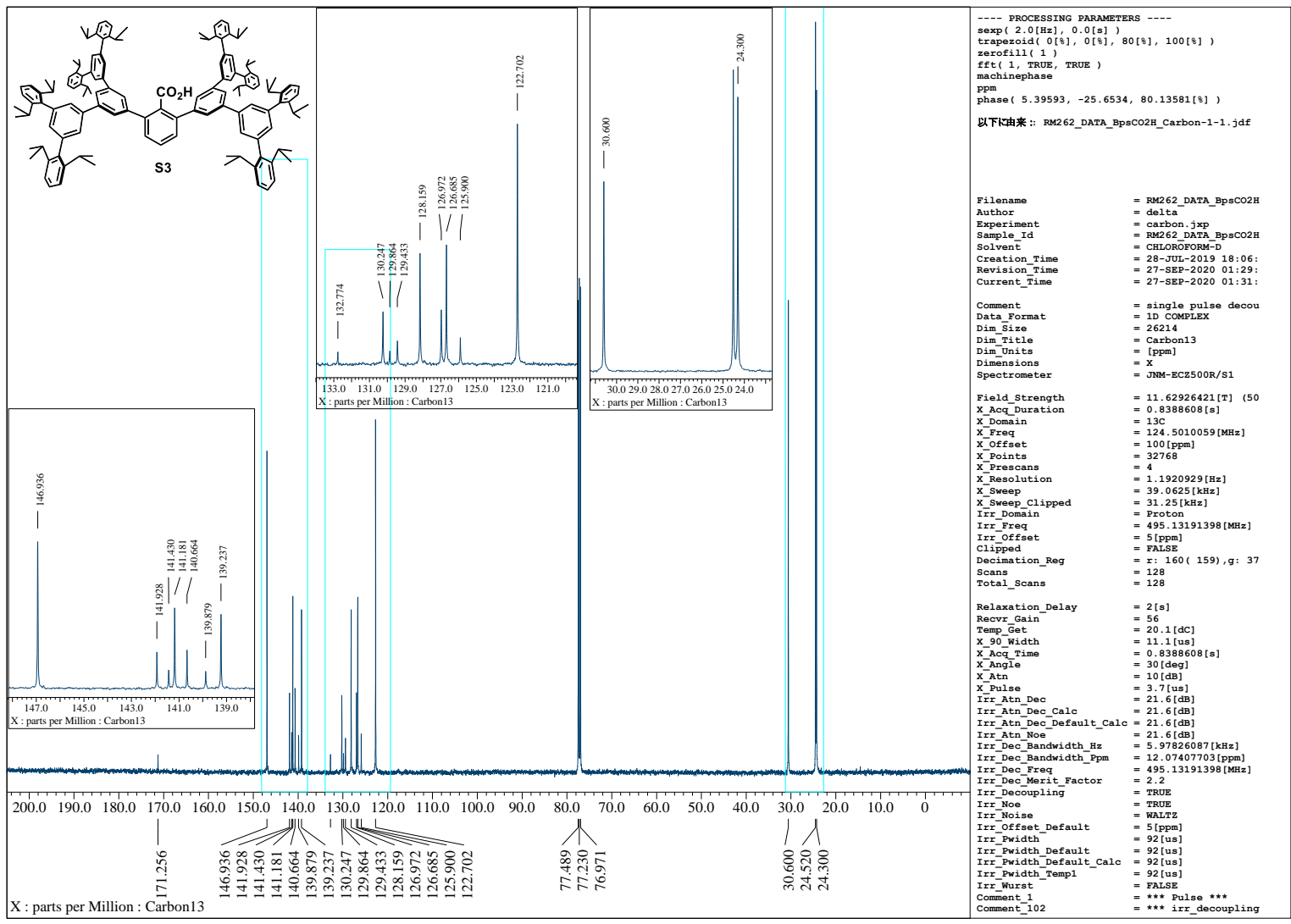

Figure S18. ${ }^{13} \mathrm{C}$ NMR $\left(125 \mathrm{MHz}, \mathrm{CDCl}_{3}\right)$ spectrum of $\mathbf{S 3}$. 


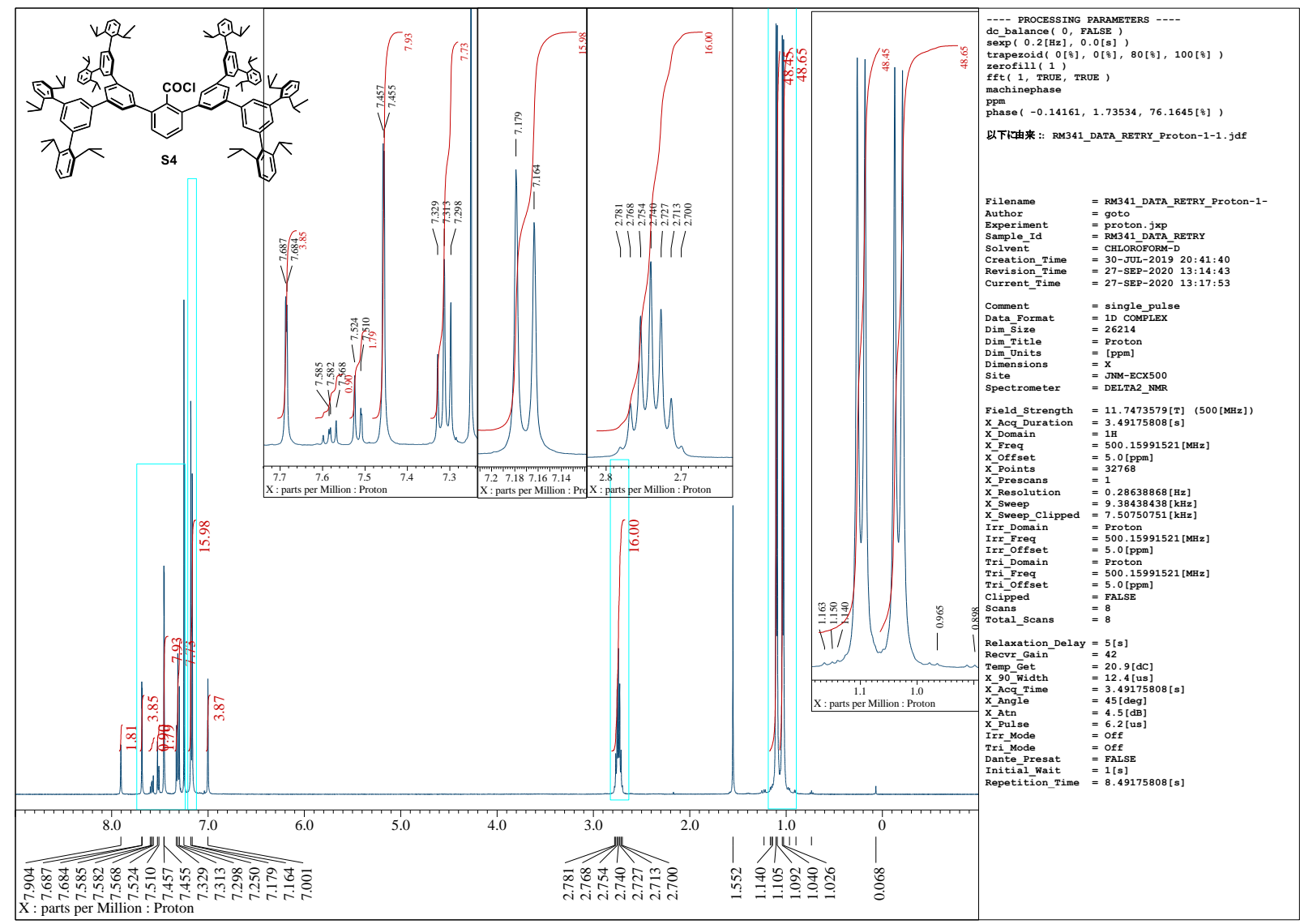

Figure S19. ${ }^{1} \mathrm{H}$ NMR $\left(500 \mathrm{MHz}, \mathrm{CDCl}_{3}\right)$ spectrum of $\mathbf{S 4}$.

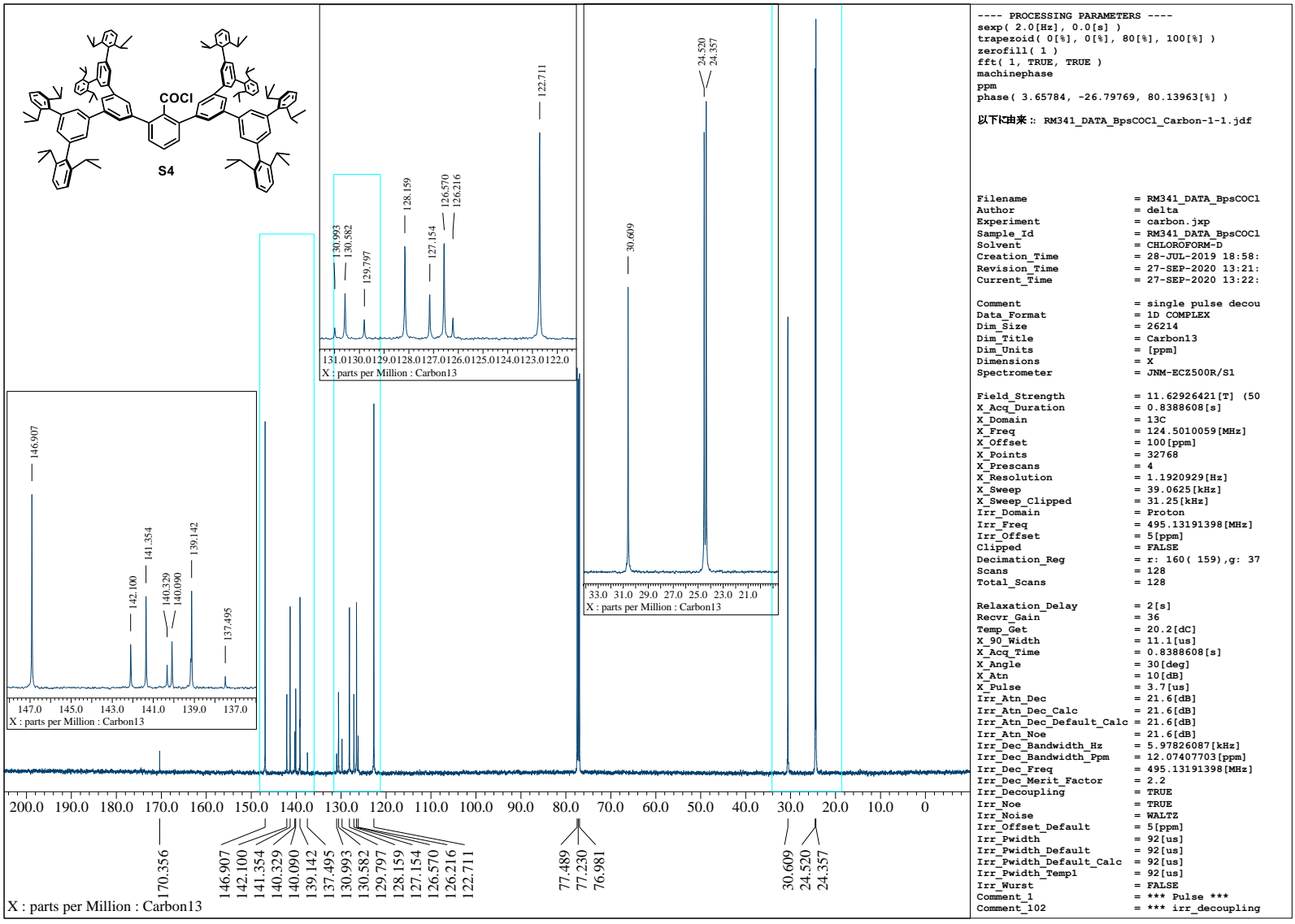

Figure S20. ${ }^{13} \mathrm{C}$ NMR $\left(125 \mathrm{MHz}, \mathrm{CDCl}_{3}\right)$ spectrum of $\mathbf{S} 4$. 


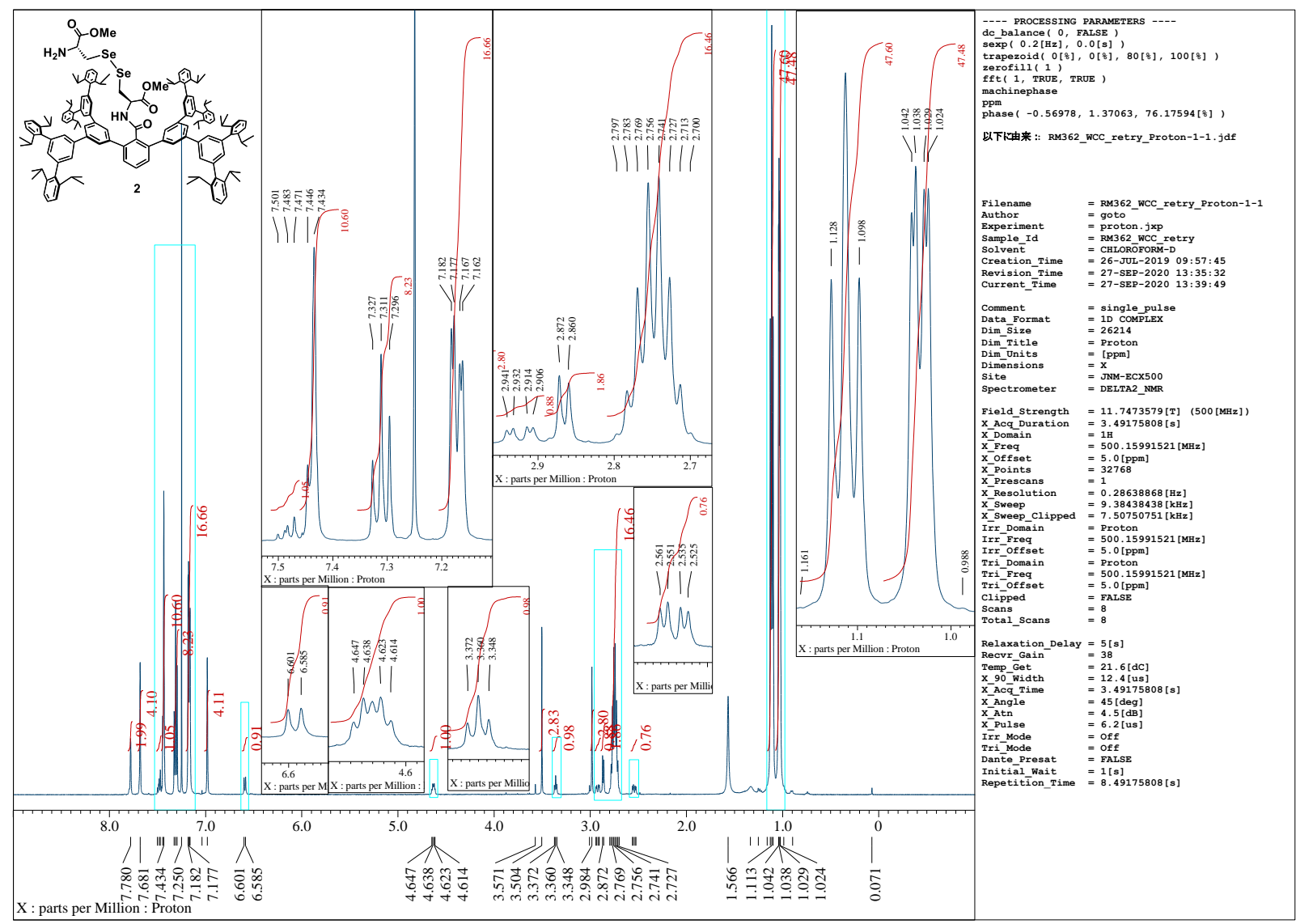

Figure S21. ${ }^{1} \mathrm{H}$ NMR $\left(500 \mathrm{MHz}, \mathrm{CDCl}_{3}\right)$ spectrum of 2.

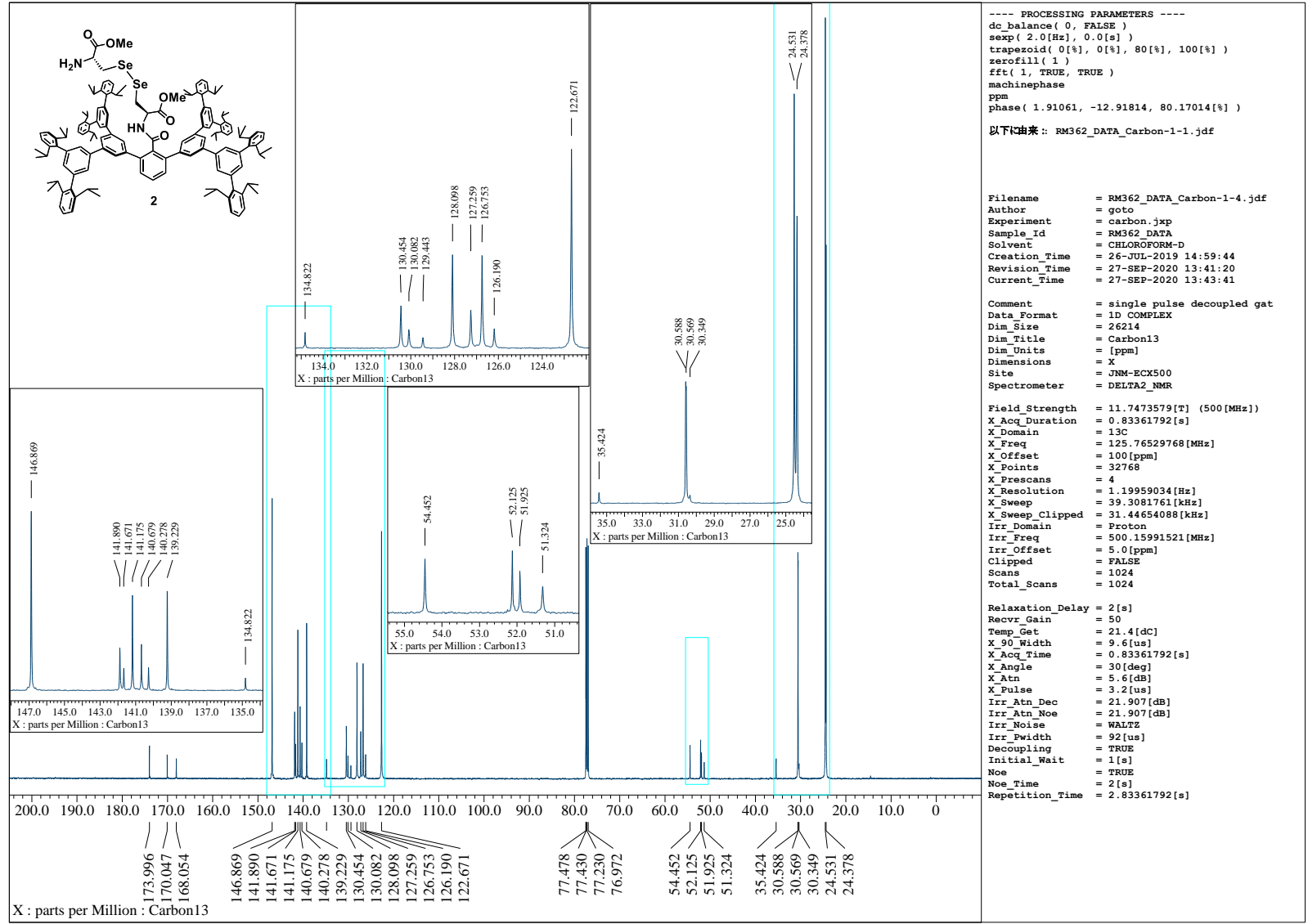

Figure S22. ${ }^{13} \mathrm{C}$ NMR $\left(125 \mathrm{MHz}, \mathrm{CDCl}_{3}\right)$ spectrum of 2. 


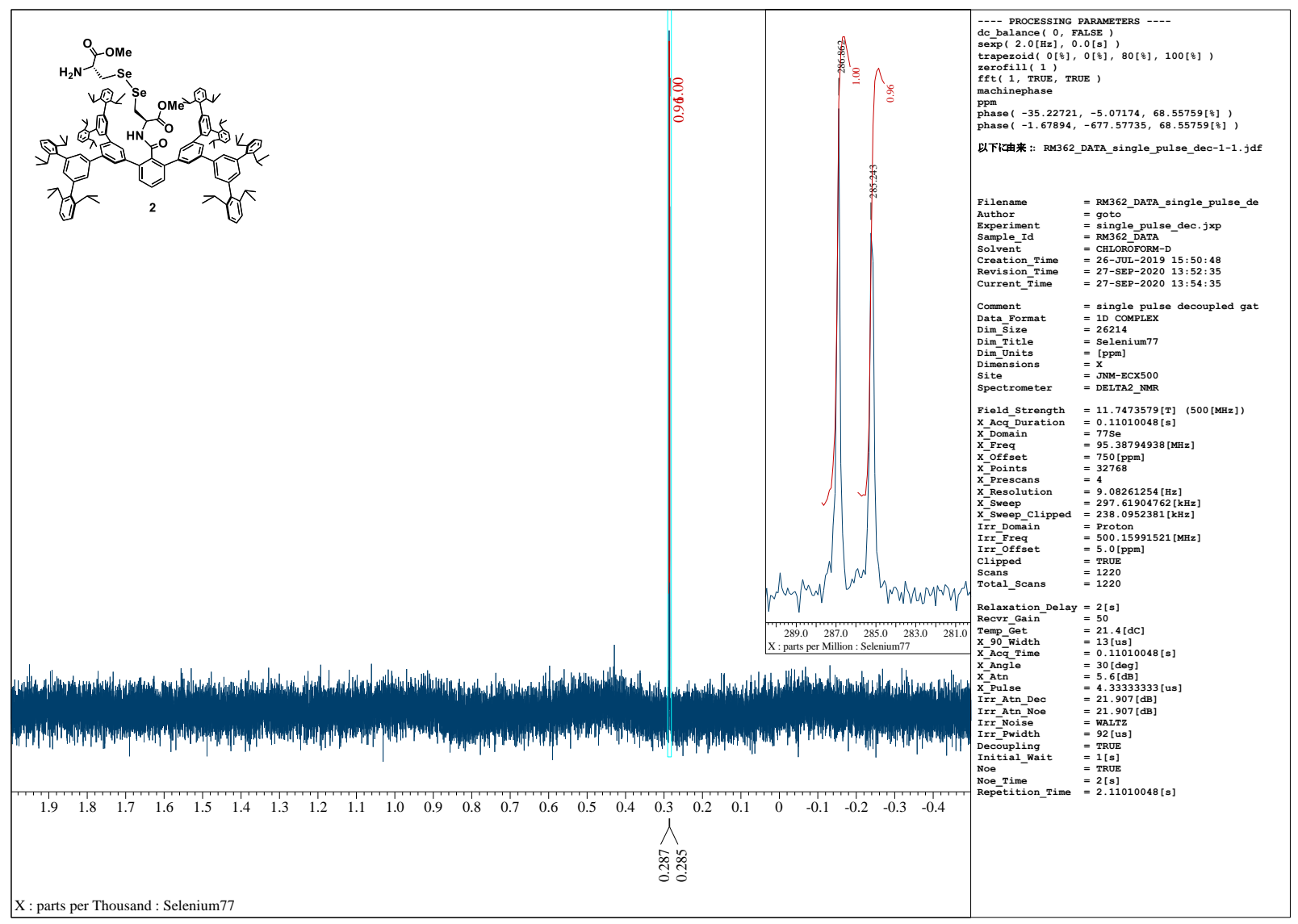

Figure S23. ${ }^{77} \mathrm{Se} \mathrm{NMR}\left(95 \mathrm{MHz}, \mathrm{CDCl}_{3}\right)$ spectrum of 2.

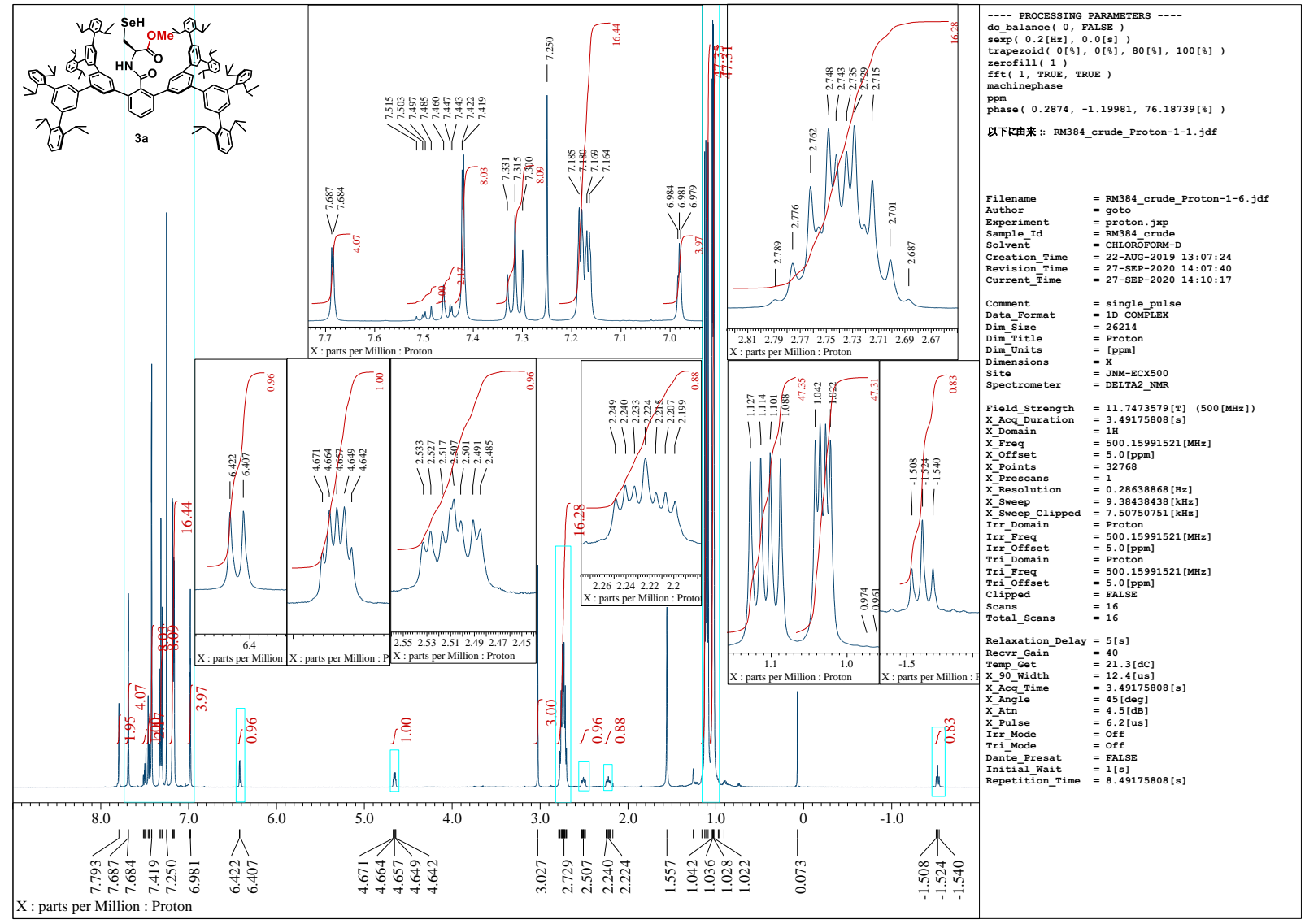

Figure S24. ${ }^{1} \mathrm{H}$ NMR $\left(500 \mathrm{MHz}, \mathrm{CDCl}_{3}\right)$ spectrum of 3a. 


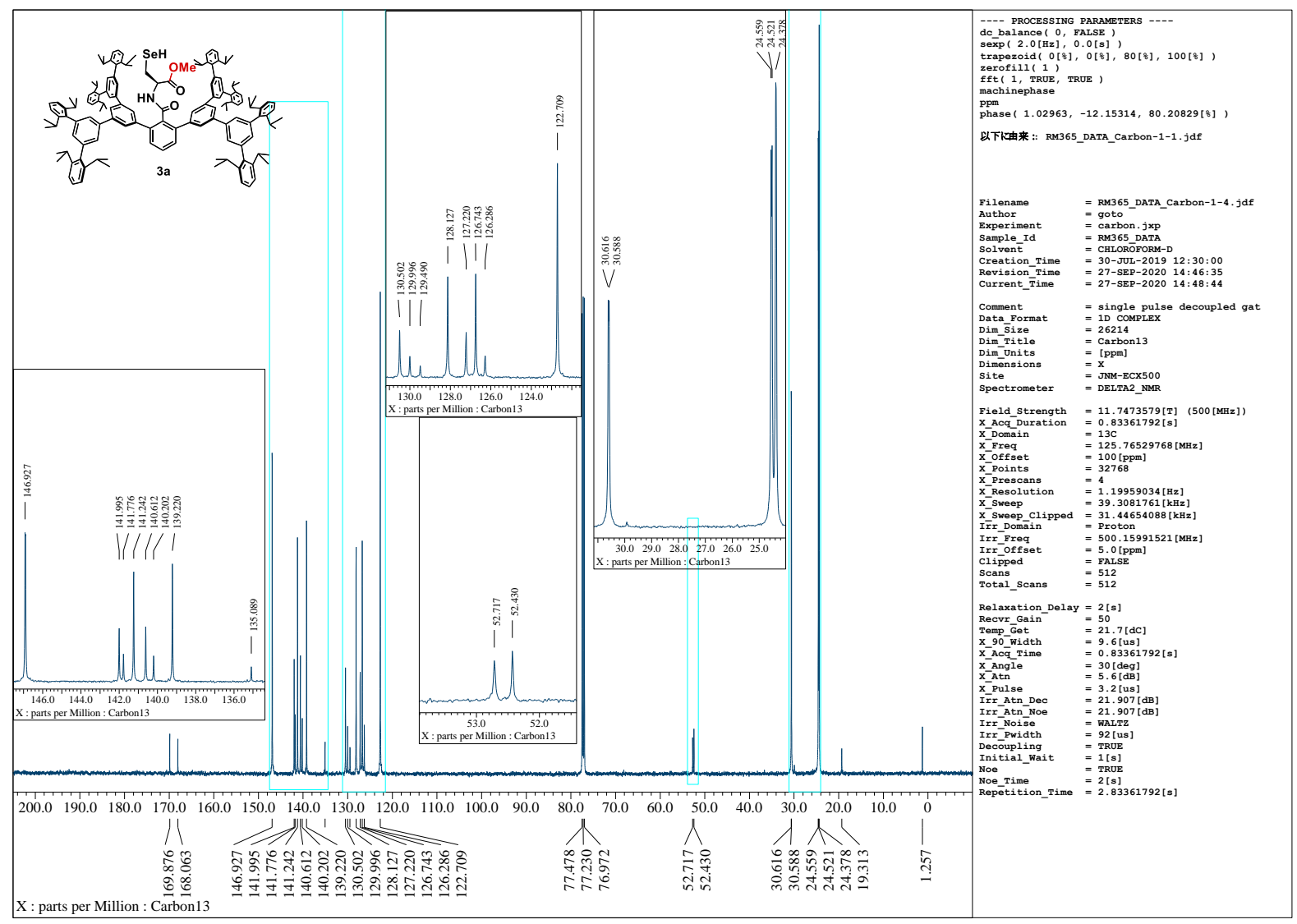

Figure S25. ${ }^{13} \mathrm{C}$ NMR $\left(125 \mathrm{MHz}, \mathrm{CDCl}_{3}\right)$ spectrum of $\mathbf{3 a}$.

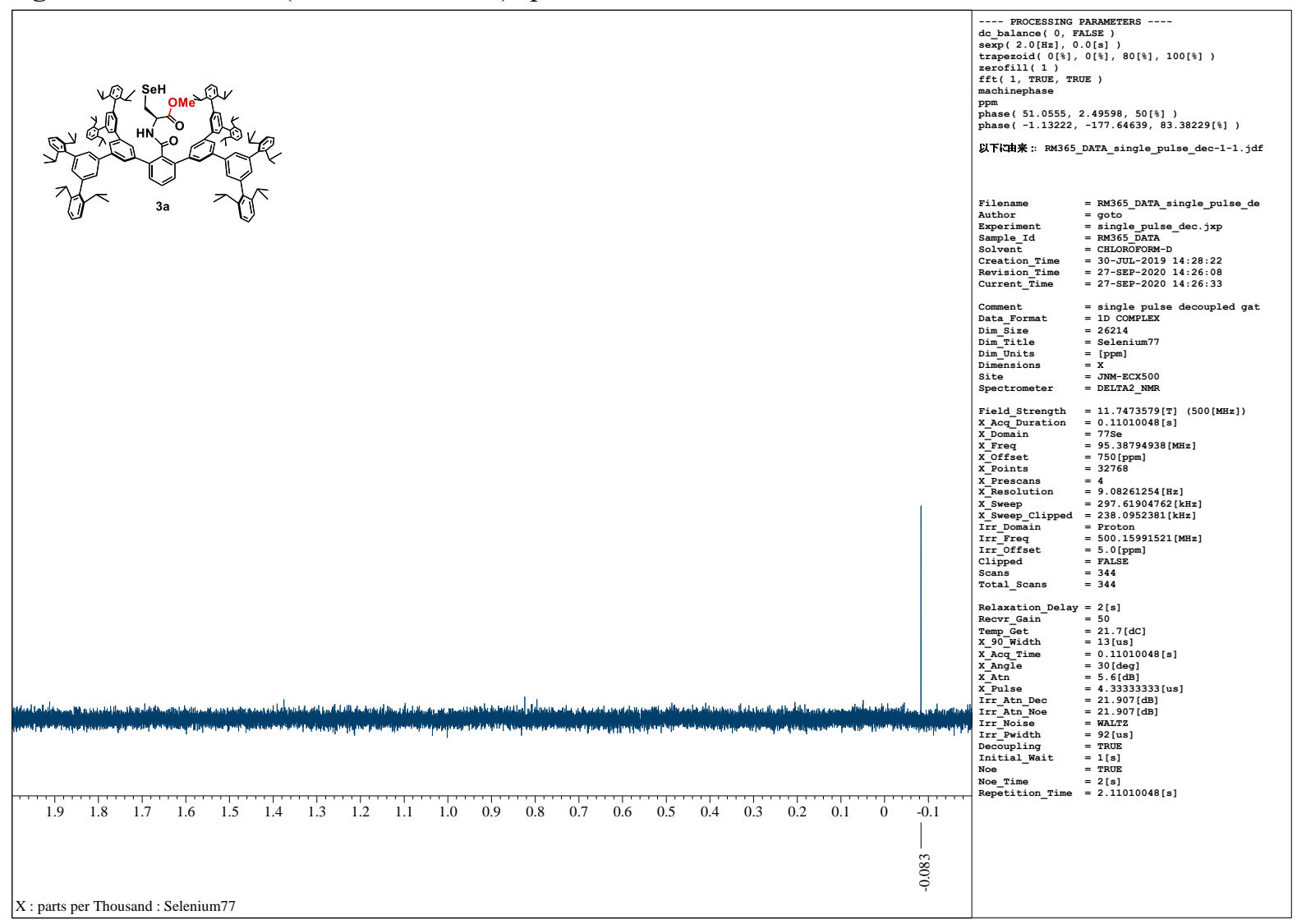

Figure S26. ${ }^{77} \mathrm{Se} \mathrm{NMR}\left(95 \mathrm{MHz}, \mathrm{CDCl}_{3}\right)$ spectrum of 3a. 


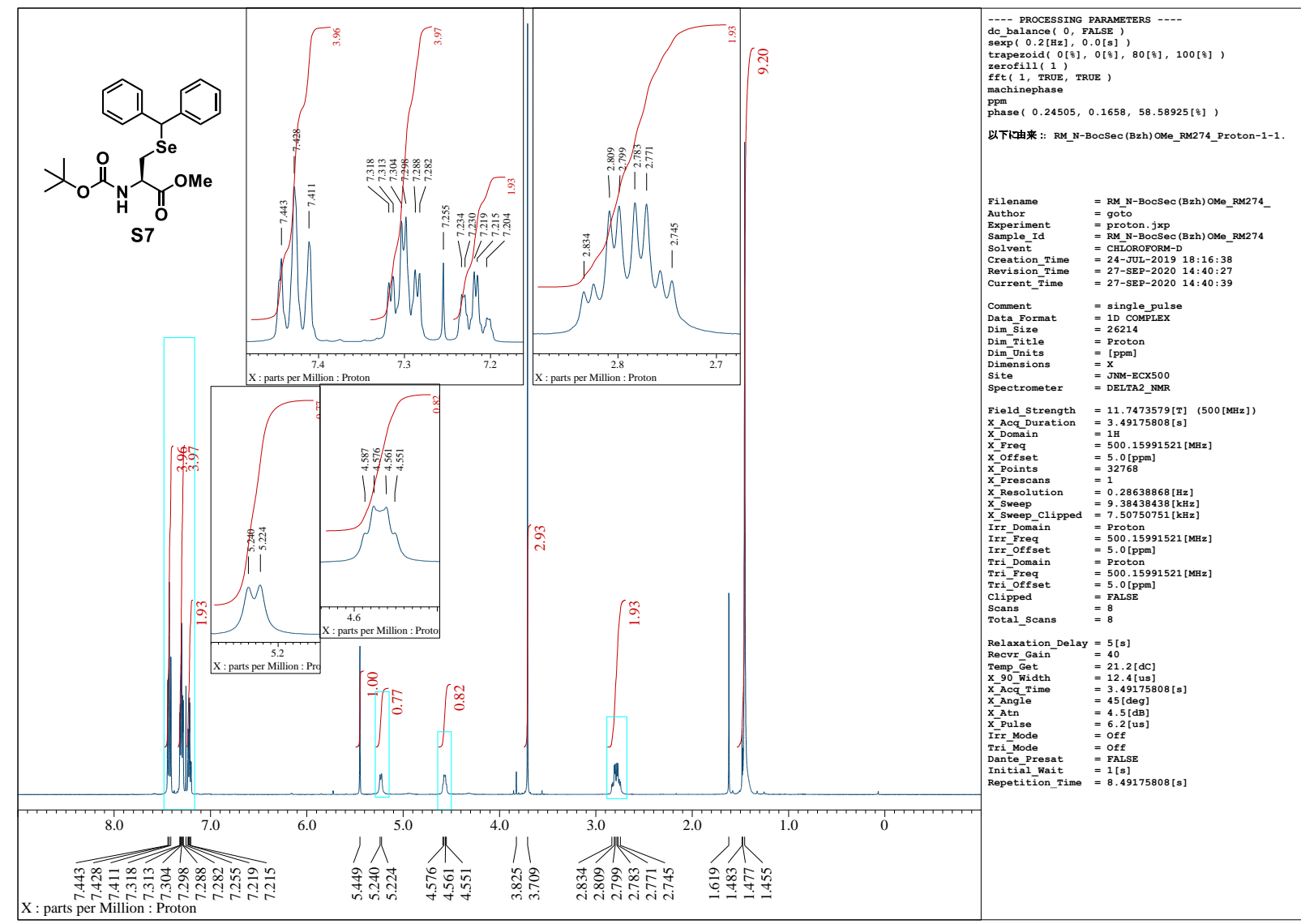

Figure S27. ${ }^{1} \mathrm{H}$ NMR $\left(500 \mathrm{MHz}, \mathrm{CDCl}_{3}\right)$ spectrum of $\mathbf{S} 7$.

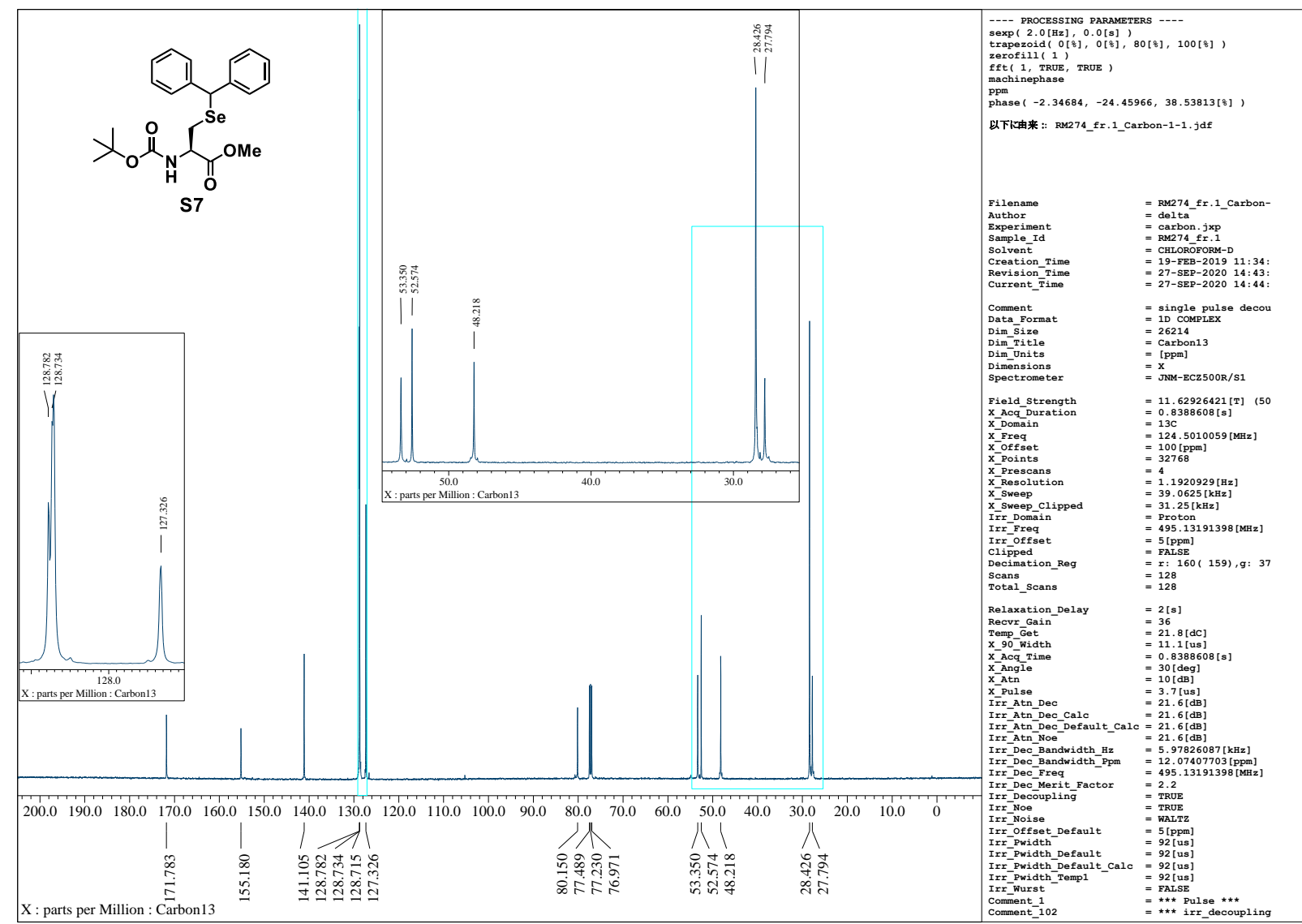

Figure S28. ${ }^{13} \mathrm{C}$ NMR $\left(125 \mathrm{MHz}, \mathrm{CDCl}_{3}\right)$ spectrum of $\mathbf{S} 7$. 


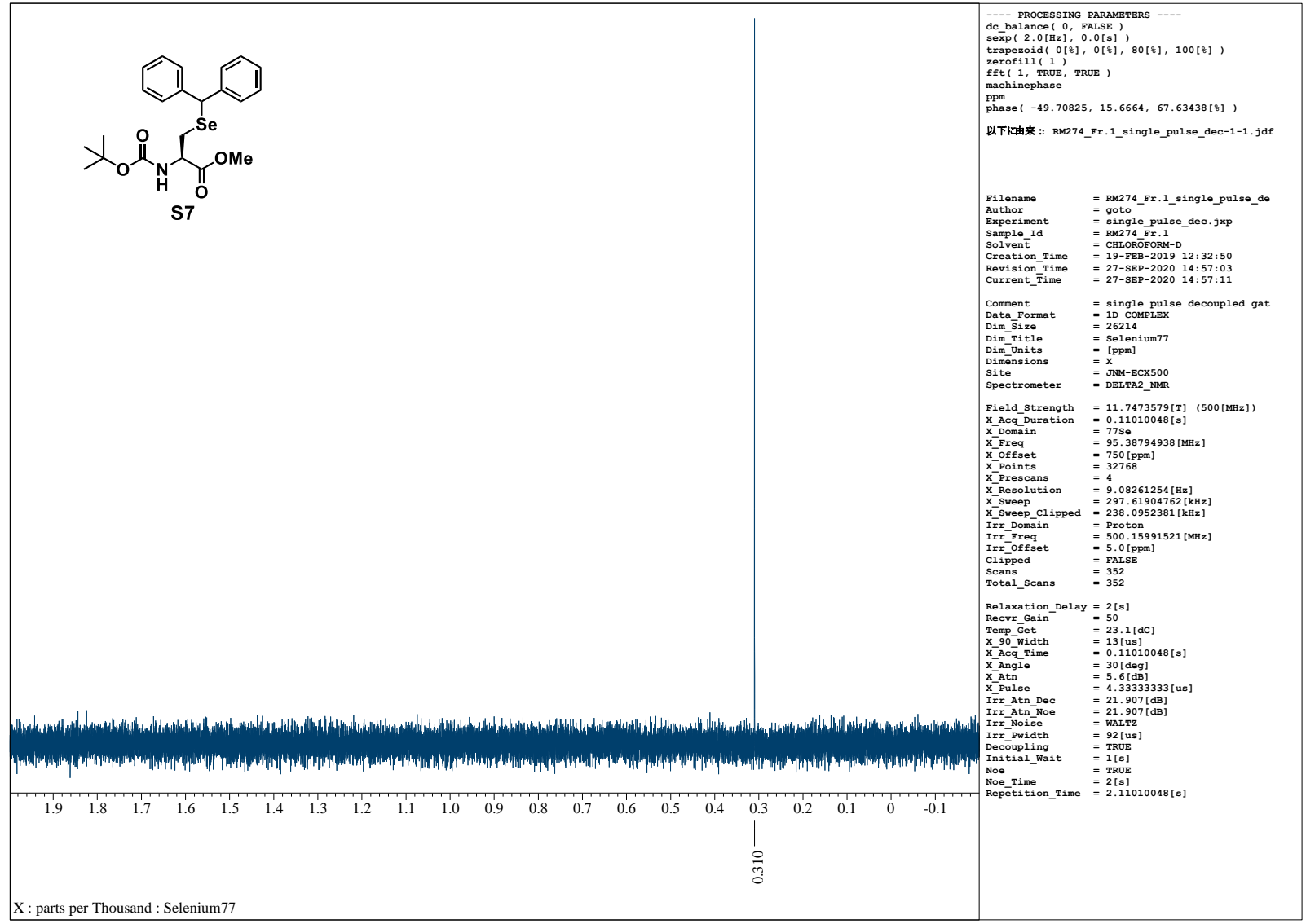

Figure S29. ${ }^{77} \mathrm{Se}$ NMR ( $95 \mathrm{MHz}, \mathrm{CDCl}_{3}$ ) spectrum of $\mathbf{S 7}$.

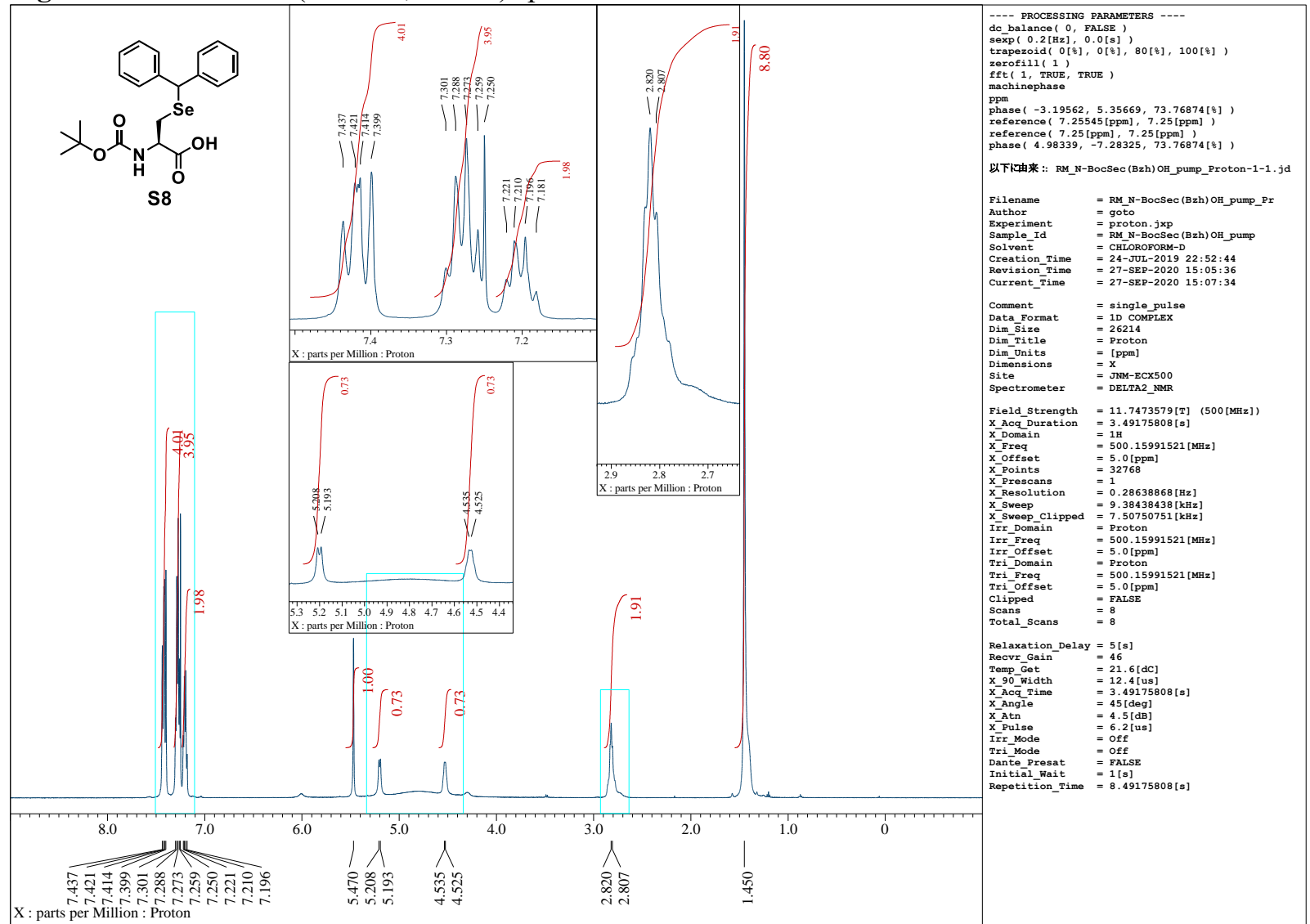

Figure S30. ${ }^{1} \mathrm{H}$ NMR $\left(500 \mathrm{MHz}, \mathrm{CDCl}_{3}\right)$ spectrum of $\mathbf{S 8}$. 


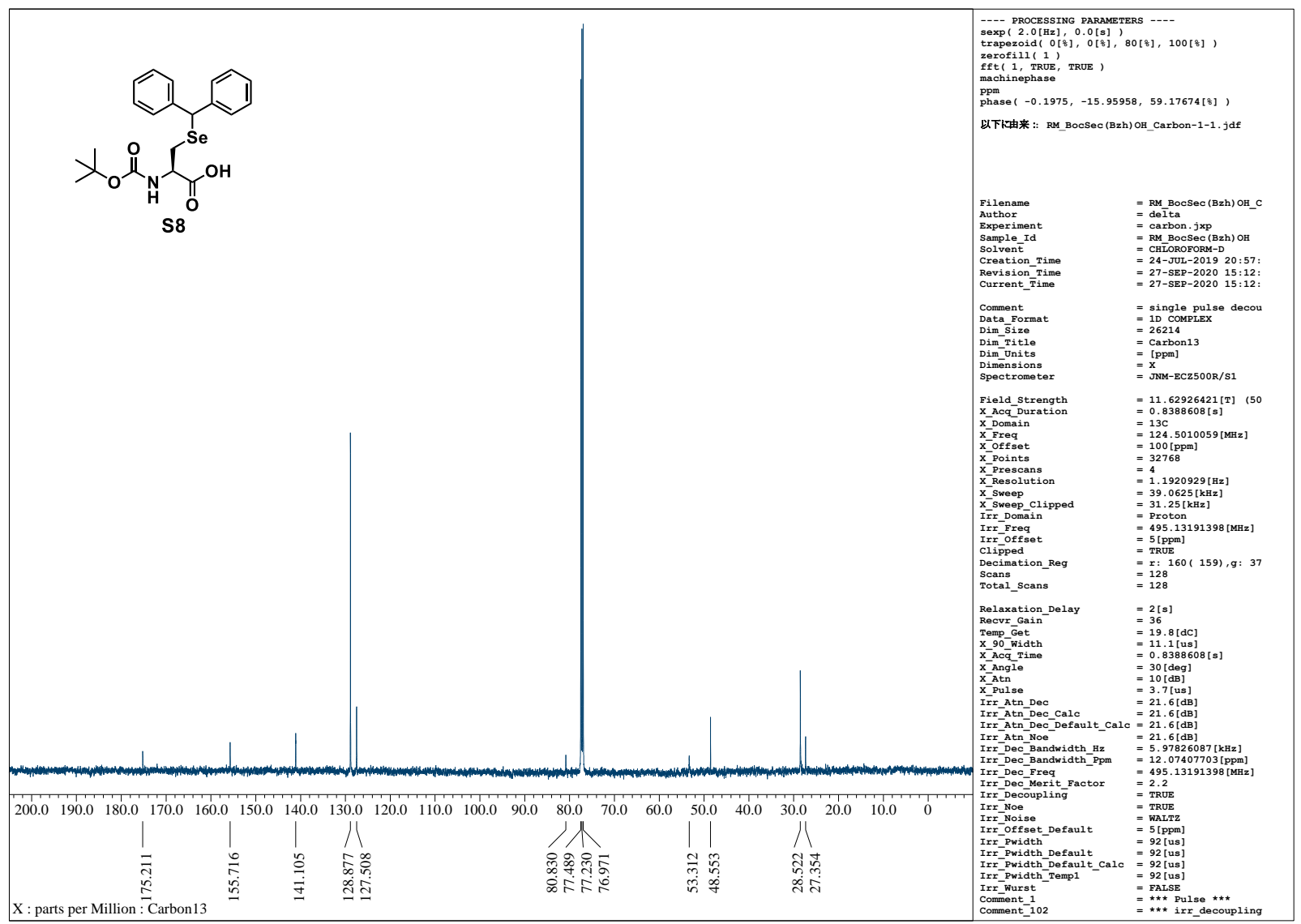

Figure S31. ${ }^{13} \mathrm{C}$ NMR $\left(125 \mathrm{MHz}, \mathrm{CDCl}_{3}\right)$ spectrum of $\mathbf{S 8}$.

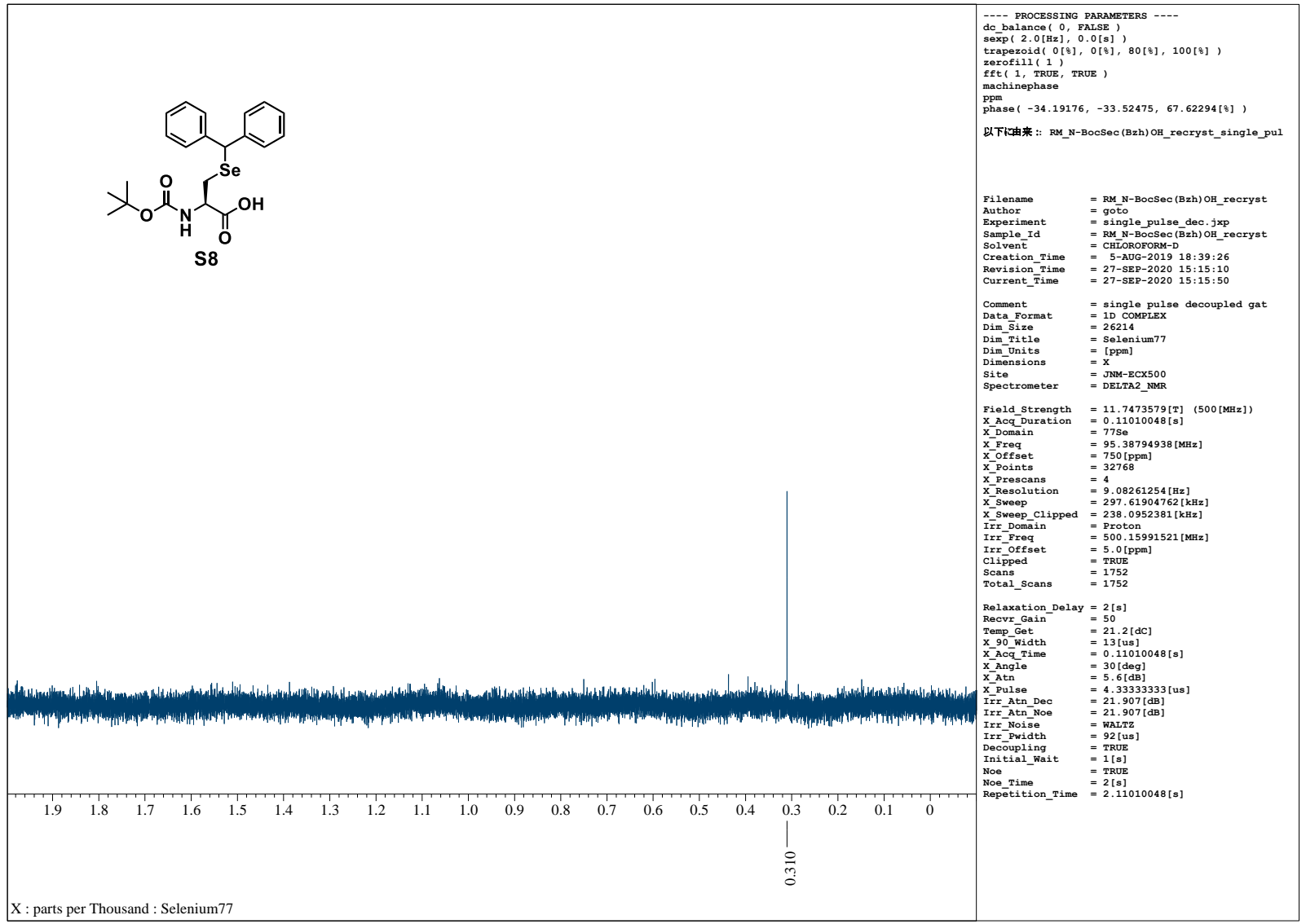

Figure S32. ${ }^{77} \mathrm{Se} \mathrm{NMR}\left(95 \mathrm{MHz}, \mathrm{CDCl}_{3}\right)$ spectrum of $\mathbf{S 8}$. 


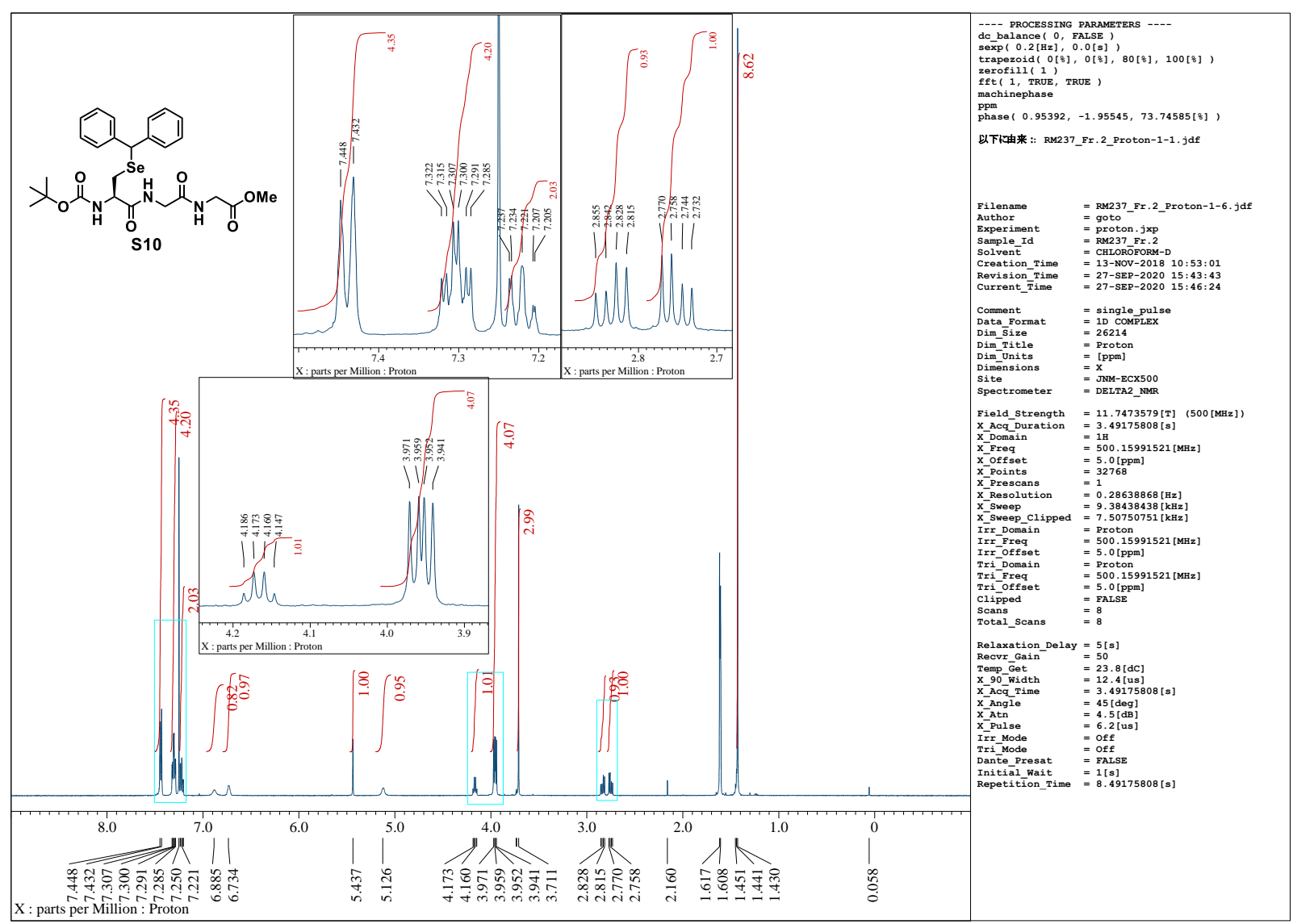

Figure S33. ${ }^{1} \mathrm{H}$ NMR $\left(500 \mathrm{MHz}, \mathrm{CDCl}_{3}\right)$ spectrum of $\mathbf{S 1 0}$.

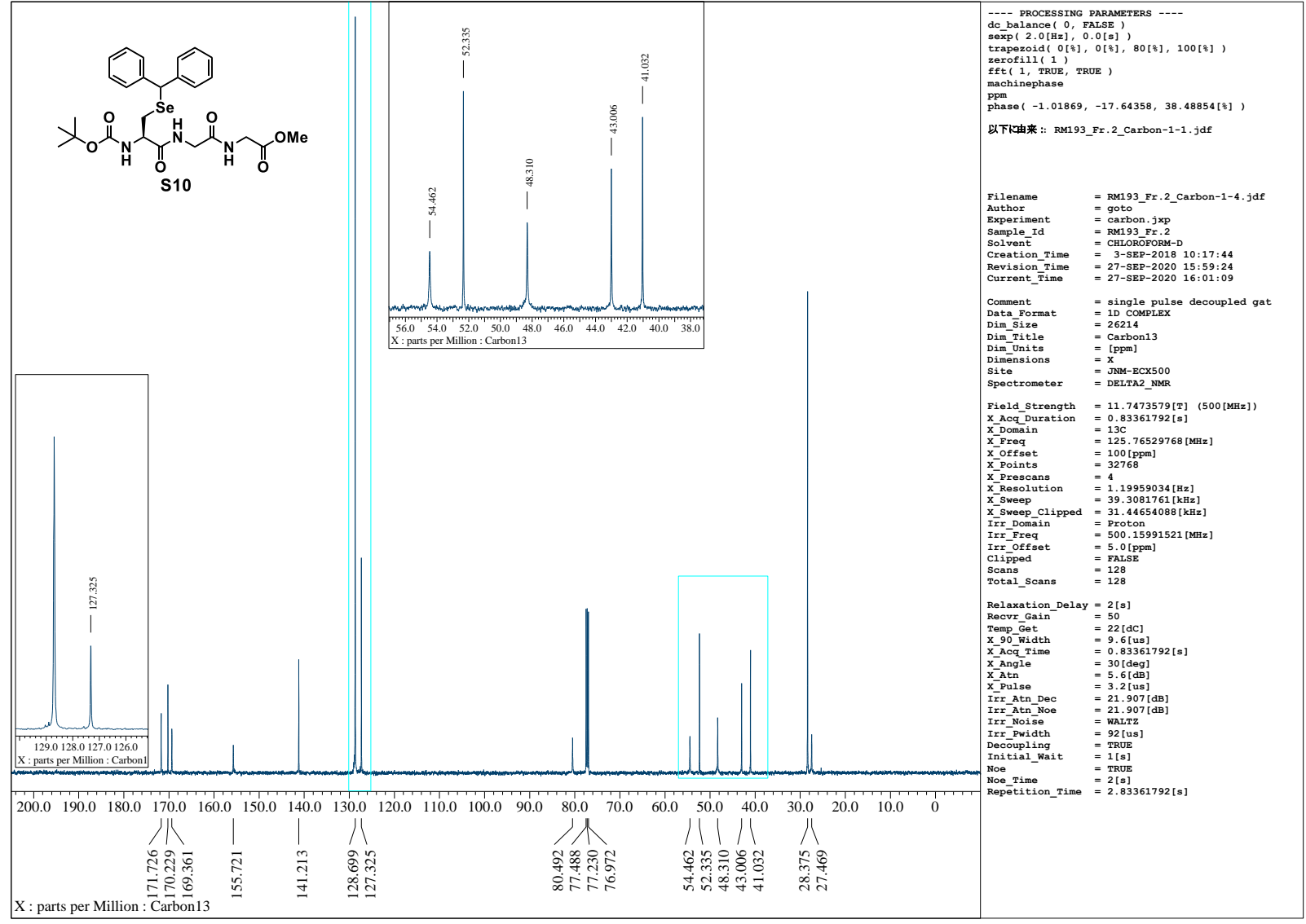

Figure S34. ${ }^{13} \mathrm{C}$ NMR $\left(125 \mathrm{MHz}, \mathrm{CDCl}_{3}\right)$ spectrum of $\mathbf{S 1 0}$. 


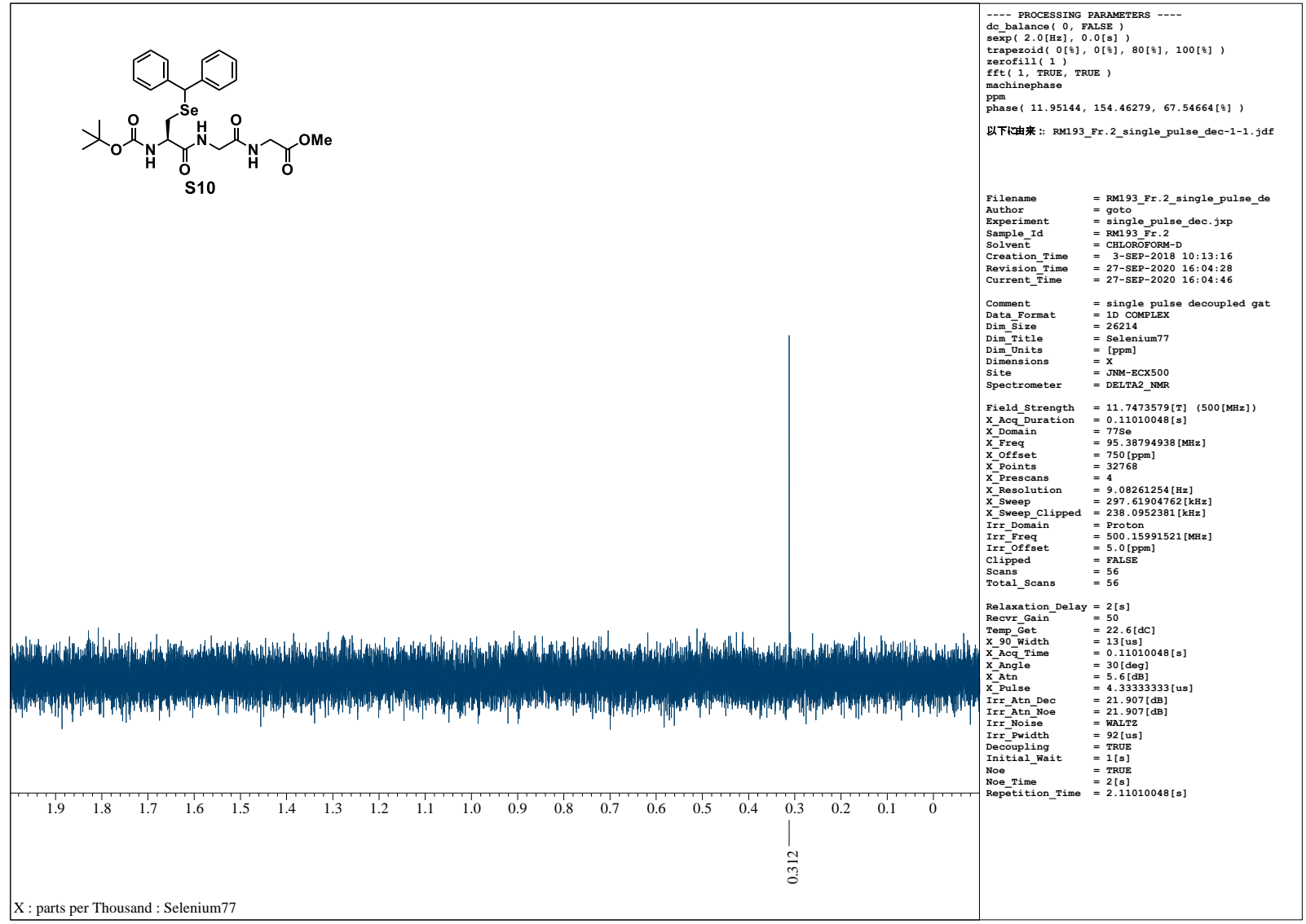

Figure S35. ${ }^{77} \mathrm{Se}$ NMR (95 MHz, $\left.\mathrm{CDCl}_{3}\right)$ spectrum of $\mathbf{S 1 0}$.

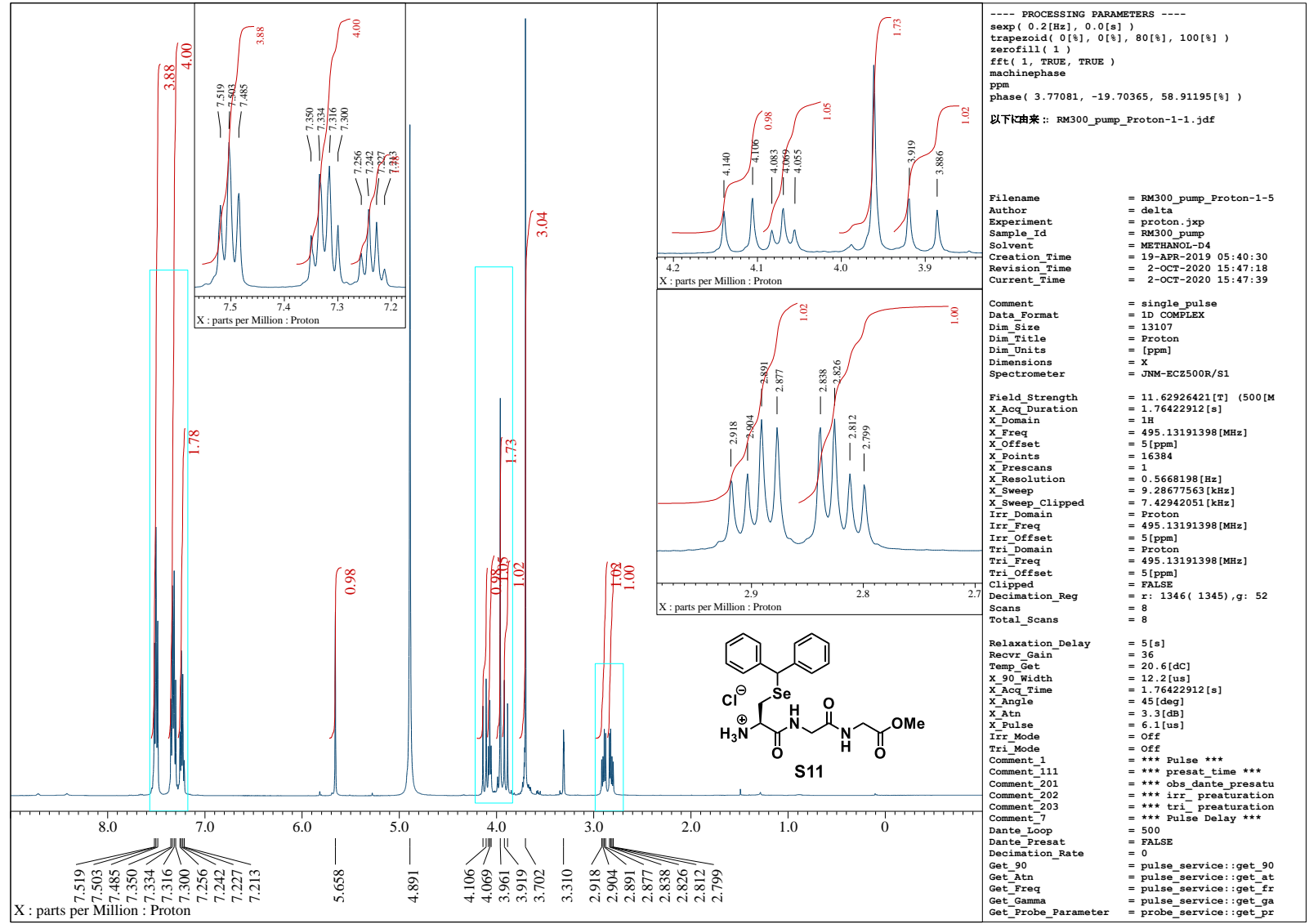

Figure S36. ${ }^{1} \mathrm{H}$ NMR $\left(500 \mathrm{MHz}, \mathrm{CD}_{3} \mathrm{OD}\right)$ spectrum of $\mathbf{S 1 1}$. 


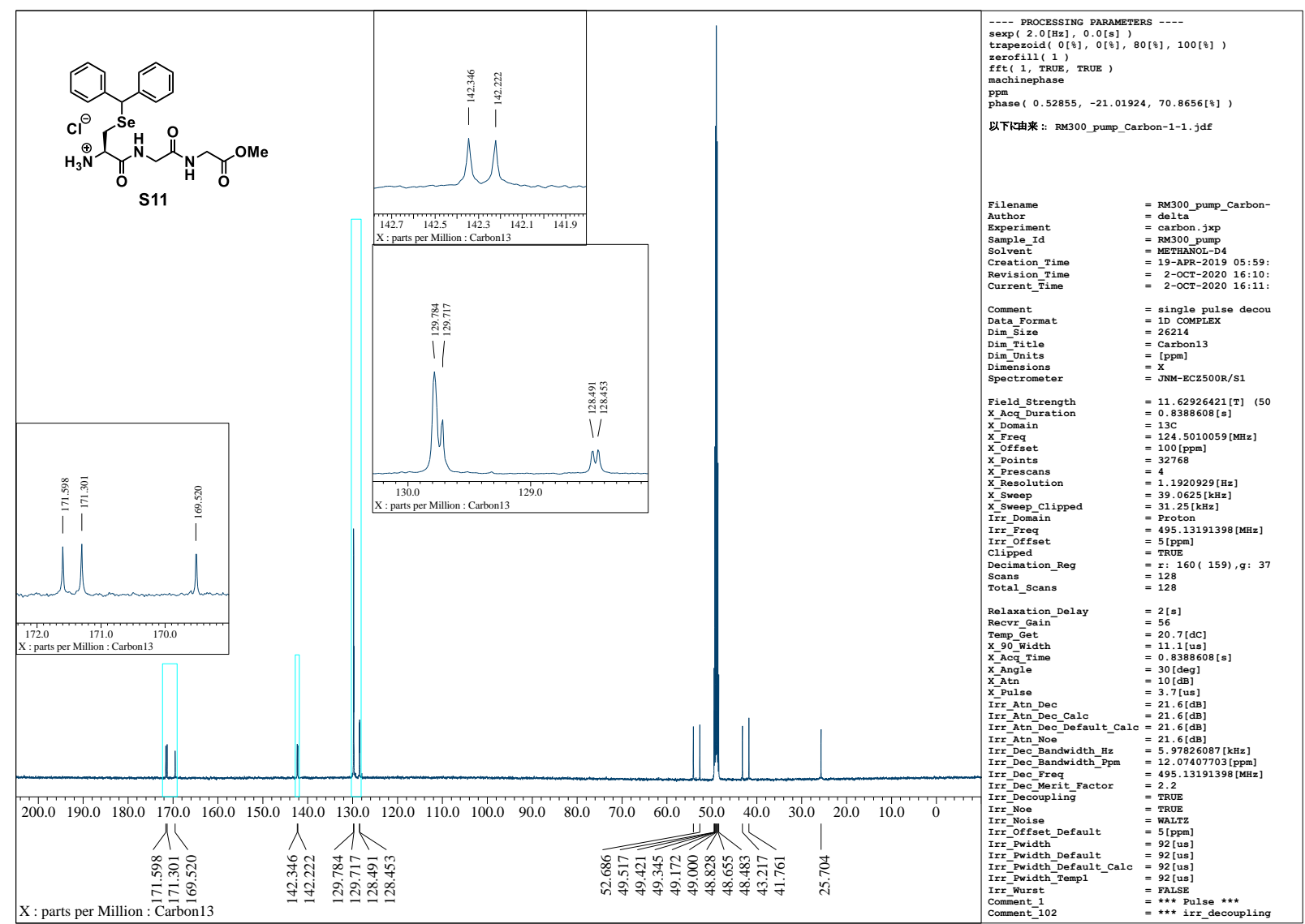

Figure S37. ${ }^{13} \mathrm{C}$ NMR $\left(125 \mathrm{MHz}, \mathrm{CD}_{3} \mathrm{OD}\right)$ spectrum of $\mathbf{S 1 1}$.

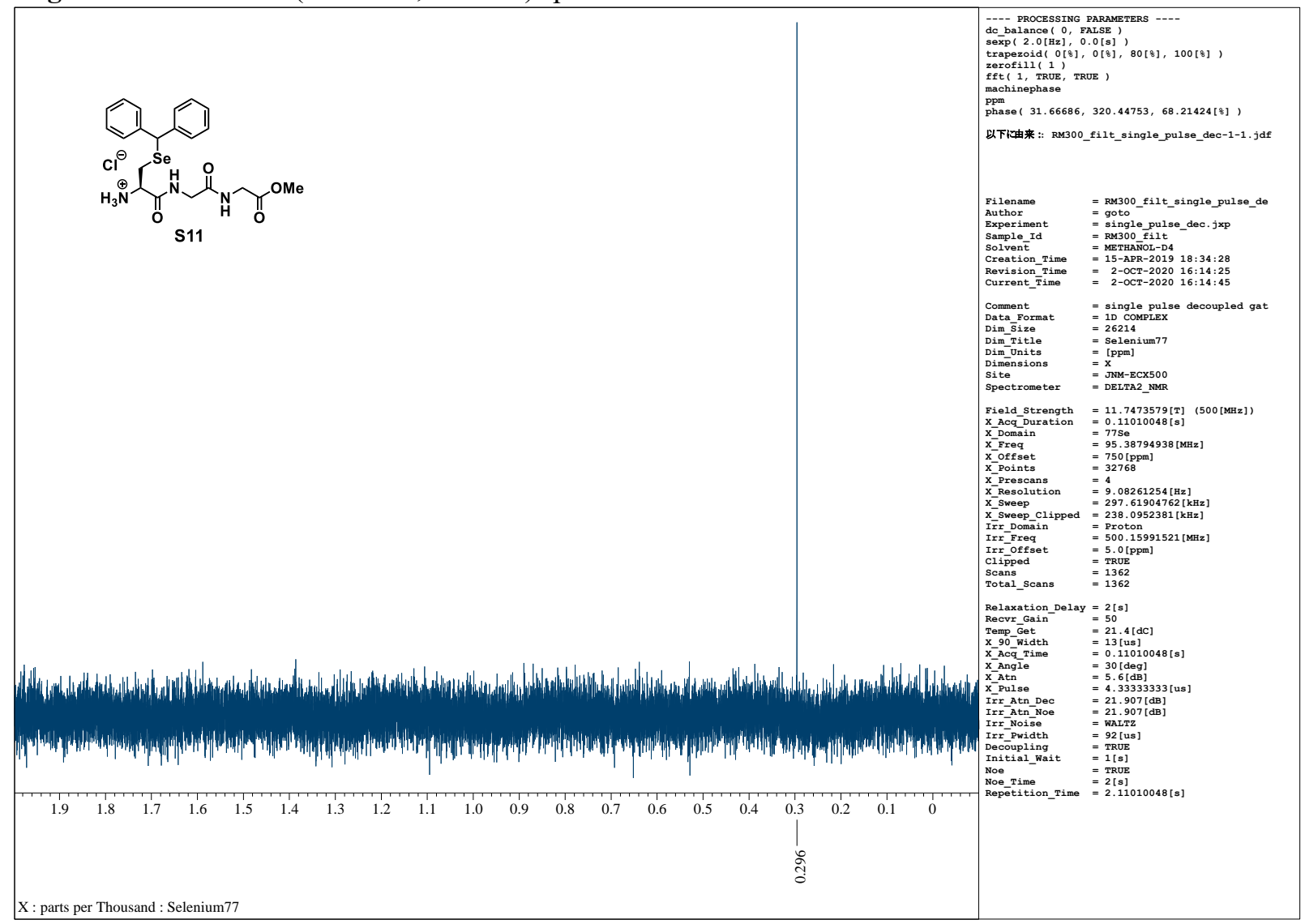

Figure S38. ${ }^{77} \mathrm{Se}$ NMR (95 MHz, $\left.\mathrm{CD}_{3} \mathrm{OD}\right)$ spectrum of $\mathbf{S 1 1}$. 


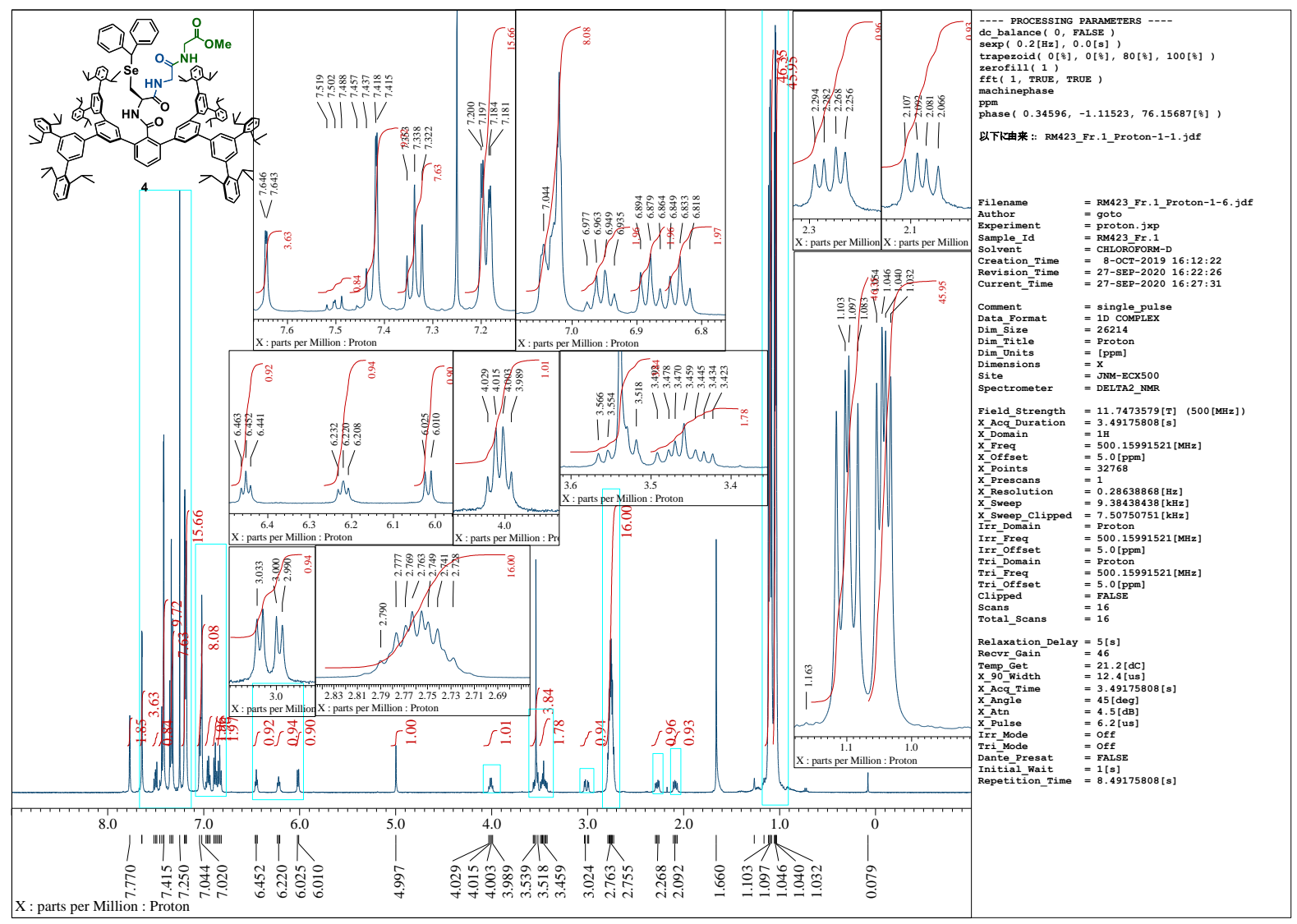

Figure S39. ${ }^{1} \mathrm{H}$ NMR $\left(500 \mathrm{MHz}, \mathrm{CDCl}_{3}\right)$ spectrum of 4.

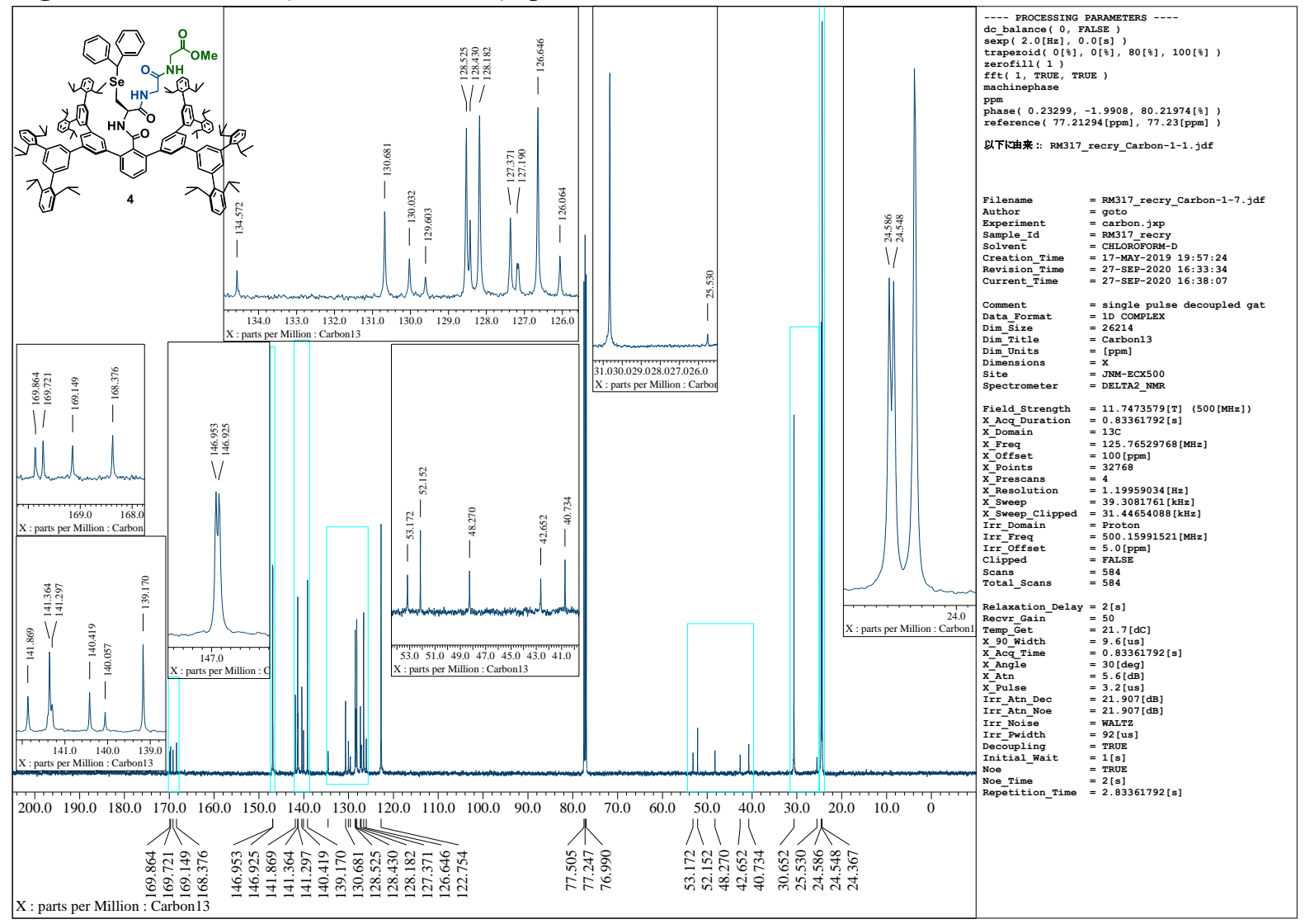

Figure S40. ${ }^{13} \mathrm{C}$ NMR $\left(125 \mathrm{MHz}, \mathrm{CDCl}_{3}\right)$ spectrum of 4 . 


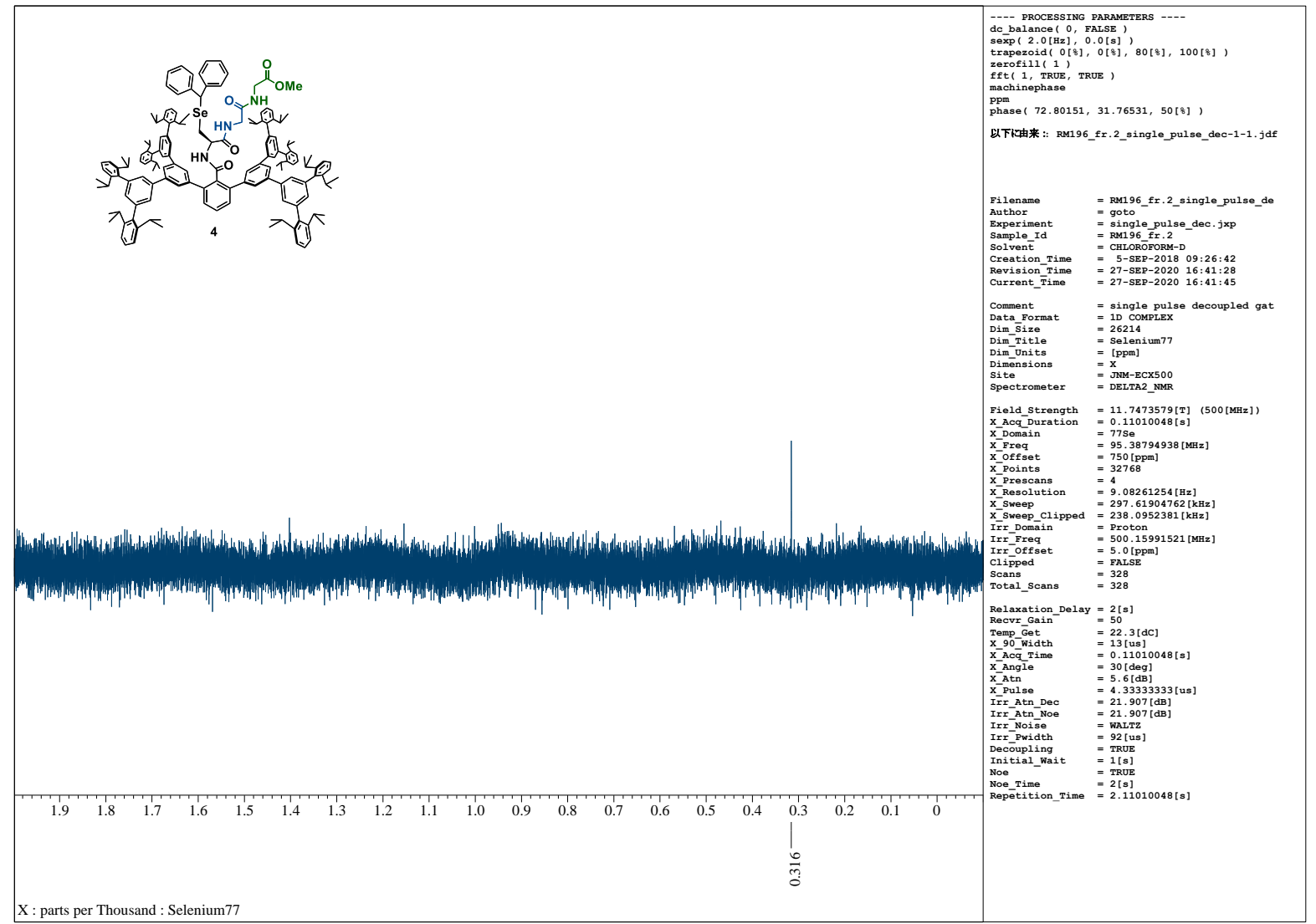

Figure S41. ${ }^{77} \mathrm{Se} \mathrm{NMR}\left(95 \mathrm{MHz}, \mathrm{CDCl}_{3}\right)$ spectrum of 4.

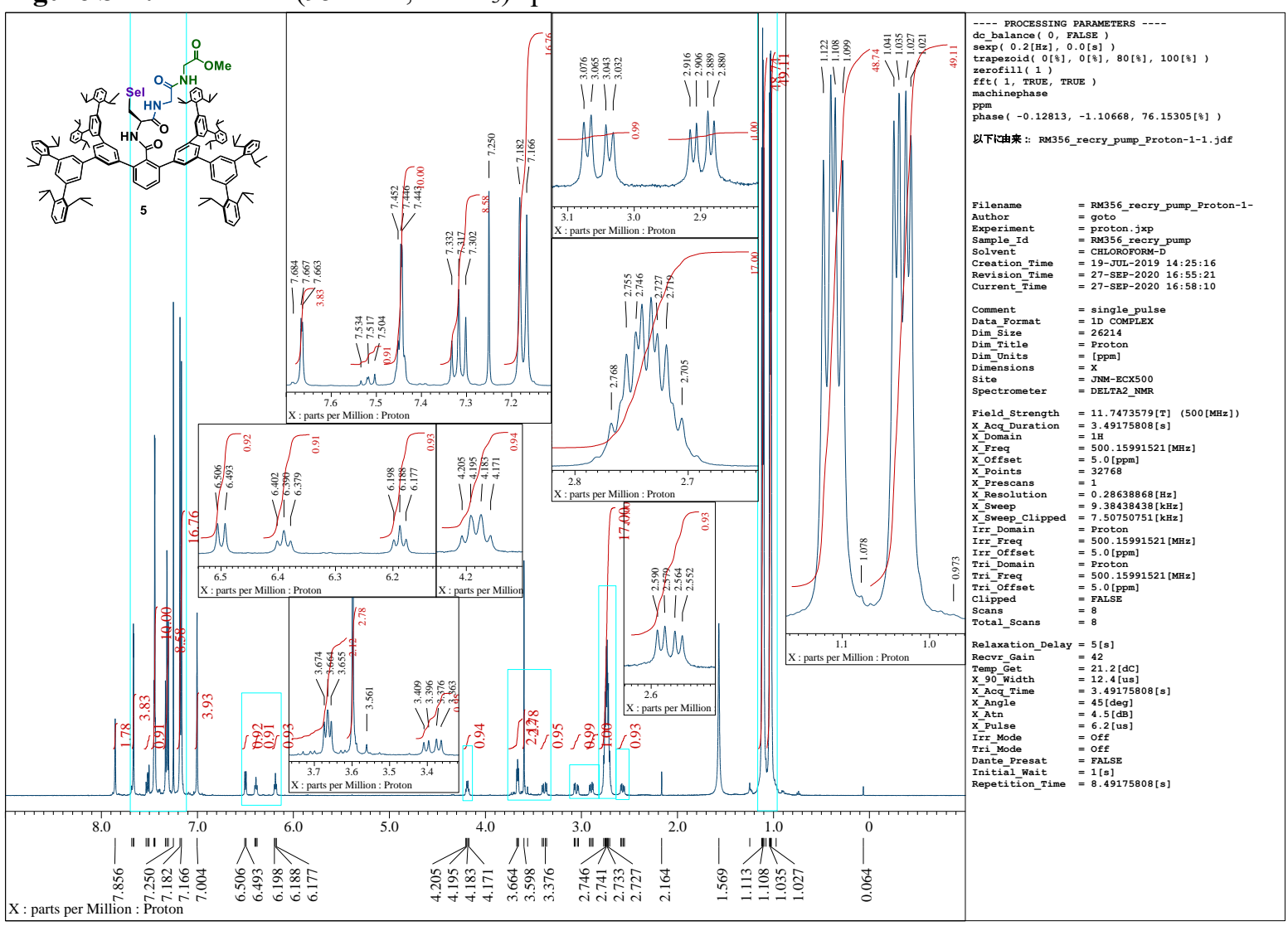

Figure S42. ${ }^{1} \mathrm{H}$ NMR $\left(500 \mathrm{MHz}, \mathrm{CDCl}_{3}\right)$ spectrum of 5 . 


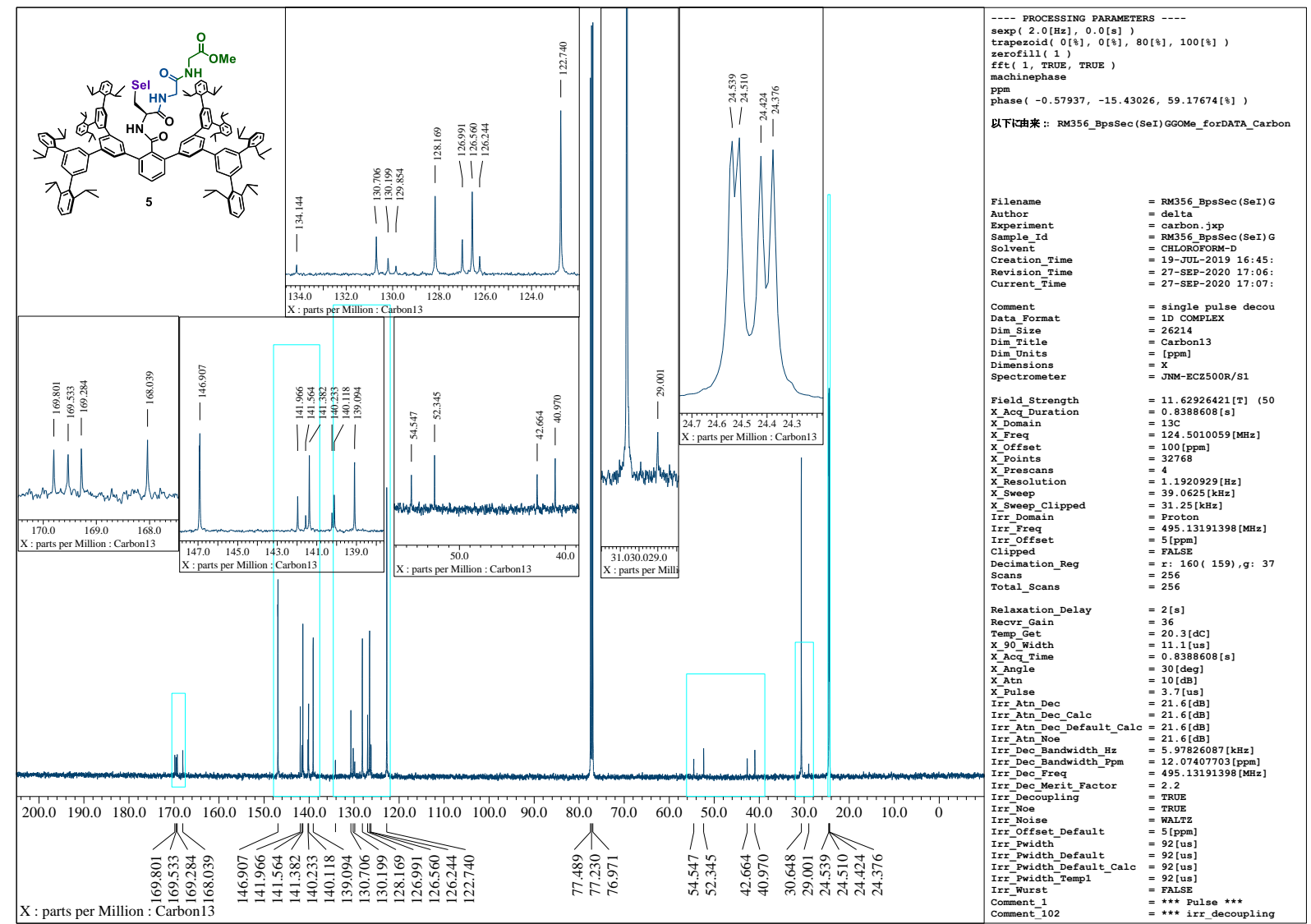

Figure S43. ${ }^{13} \mathrm{C}$ NMR (125 MHz, $\left.\mathrm{CDCl}_{3}\right)$ spectrum of 5.

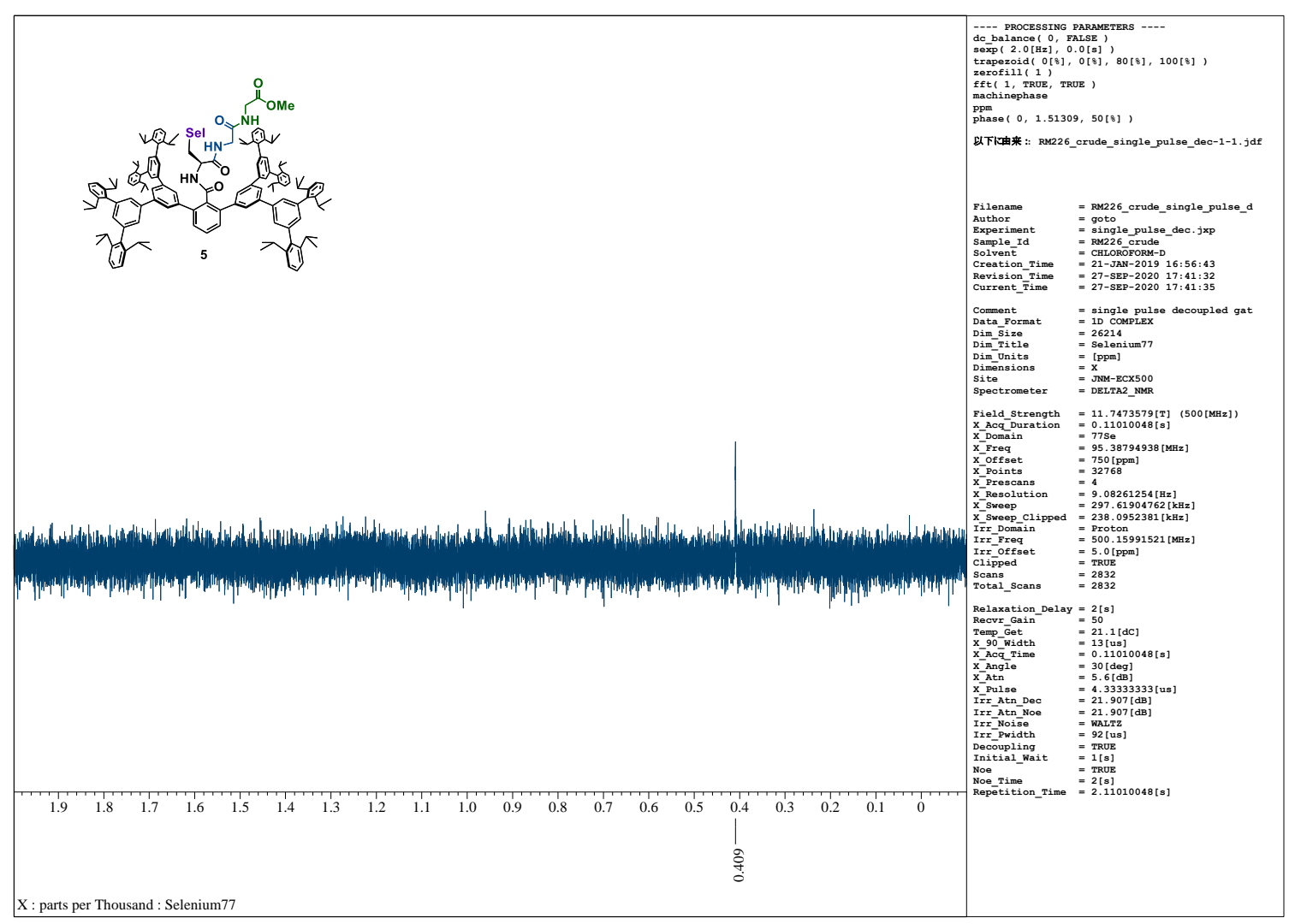

Figure S44. ${ }^{77} \mathrm{Se} \mathrm{NMR}\left(95 \mathrm{MHz}, \mathrm{CDCl}_{3}\right)$ spectrum of 5. 


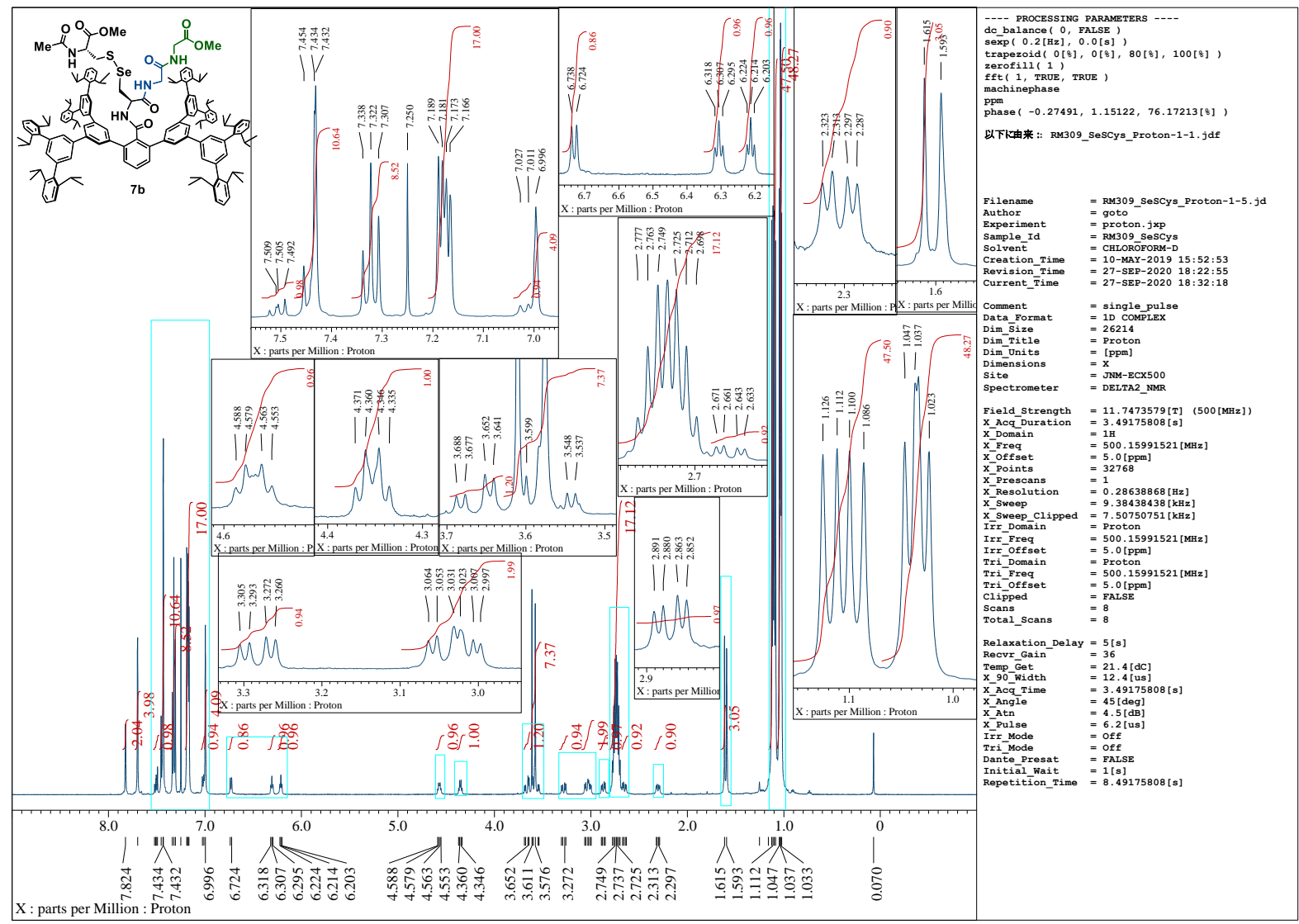

Figure S45. ${ }^{1} \mathrm{H}$ NMR $\left(500 \mathrm{MHz}, \mathrm{CDCl}_{3}\right)$ spectrum of $\mathbf{7 b}$.

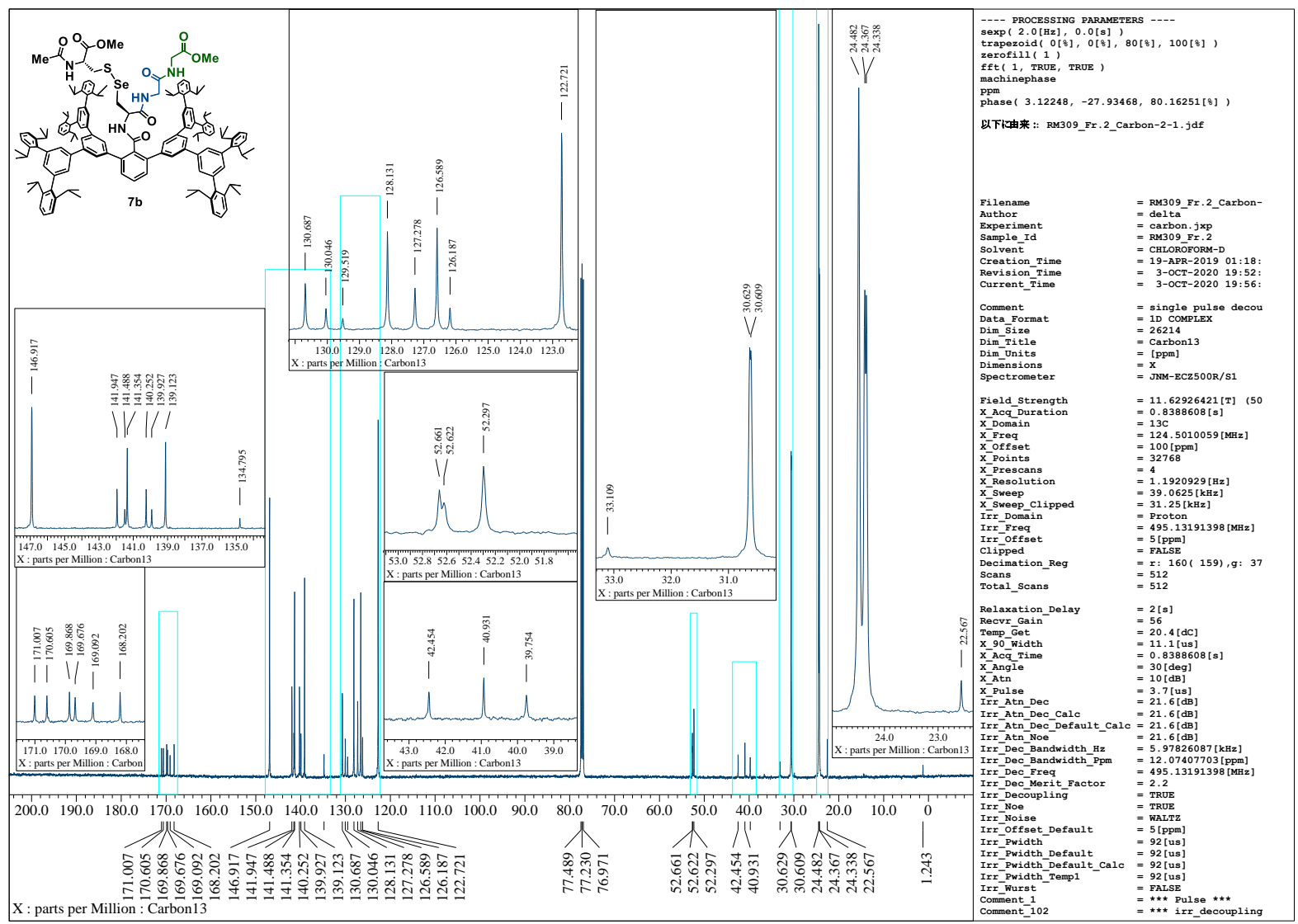

Figure S46. ${ }^{13} \mathrm{C}$ NMR (125 MHz, $\left.\mathrm{CDCl}_{3}\right)$ spectrum of $\mathbf{7 b}$. 


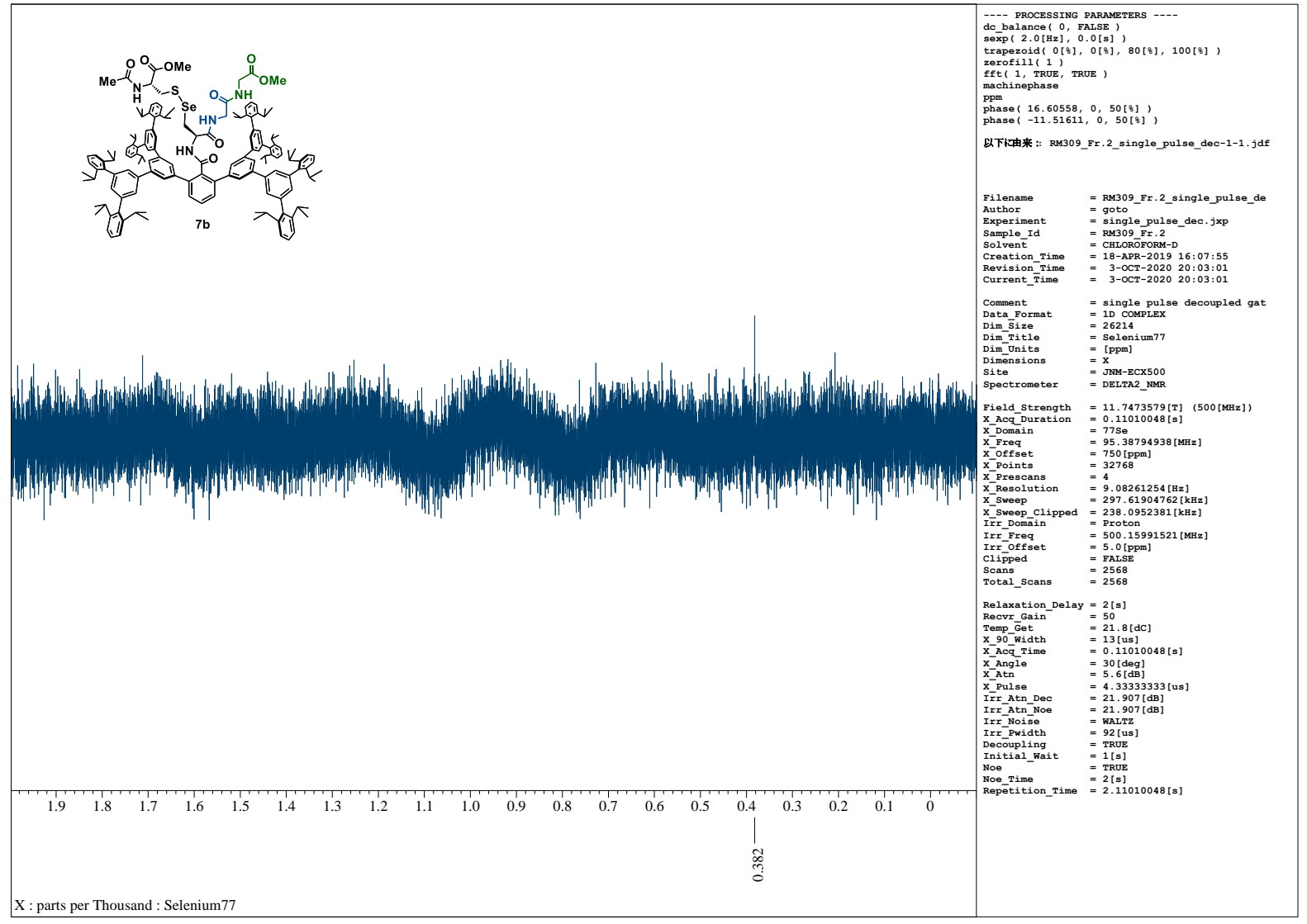

Figure S47. ${ }^{77} \mathrm{Se} \mathrm{NMR}\left(95 \mathrm{MHz}, \mathrm{CDCl}_{3}\right)$ spectrum of $\mathbf{7 b}$.

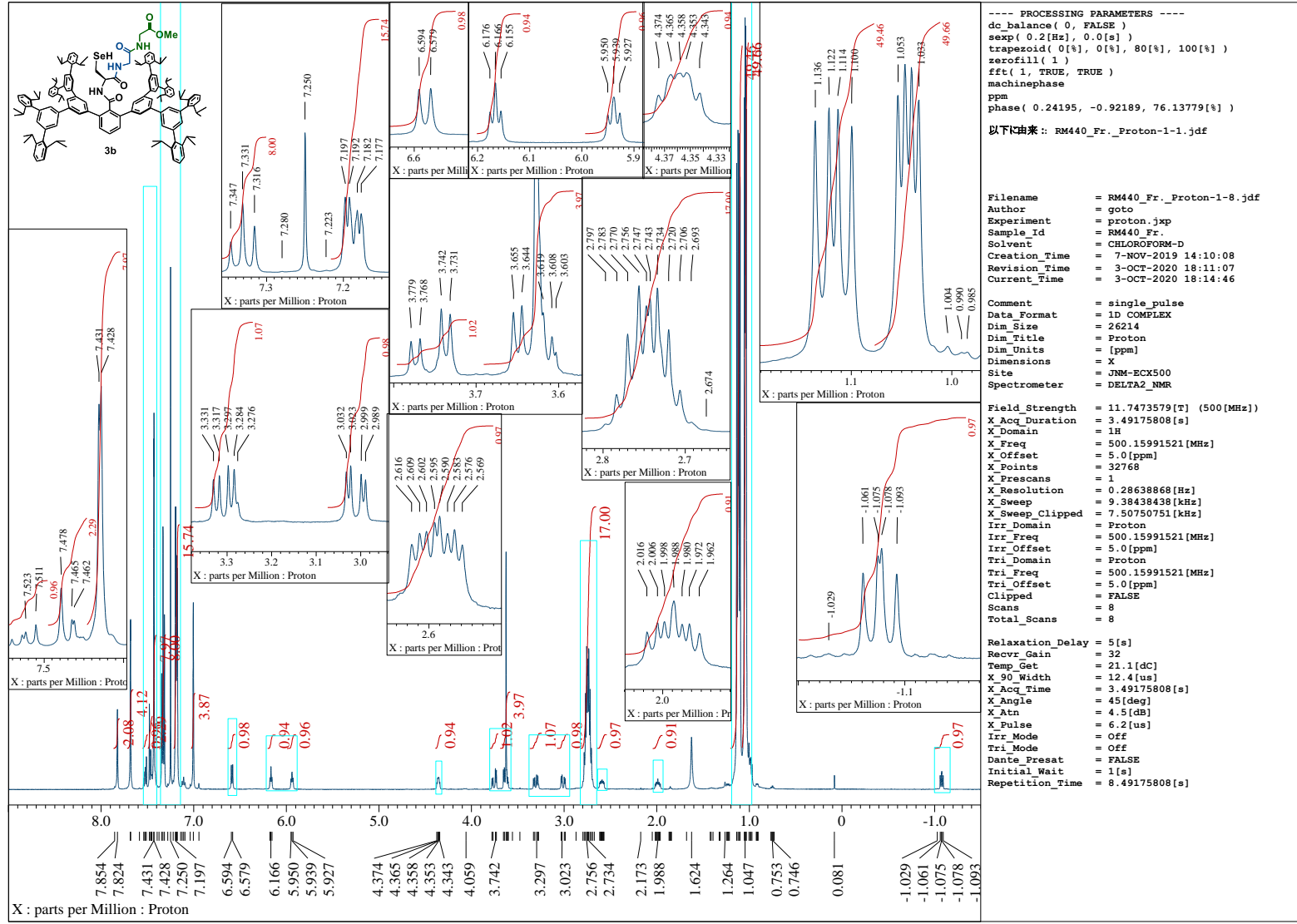

Figure S48. ${ }^{1} \mathrm{H}$ NMR $\left(500 \mathrm{MHz}, \mathrm{CDCl}_{3}\right)$ spectrum of $\mathbf{3 b}$. 


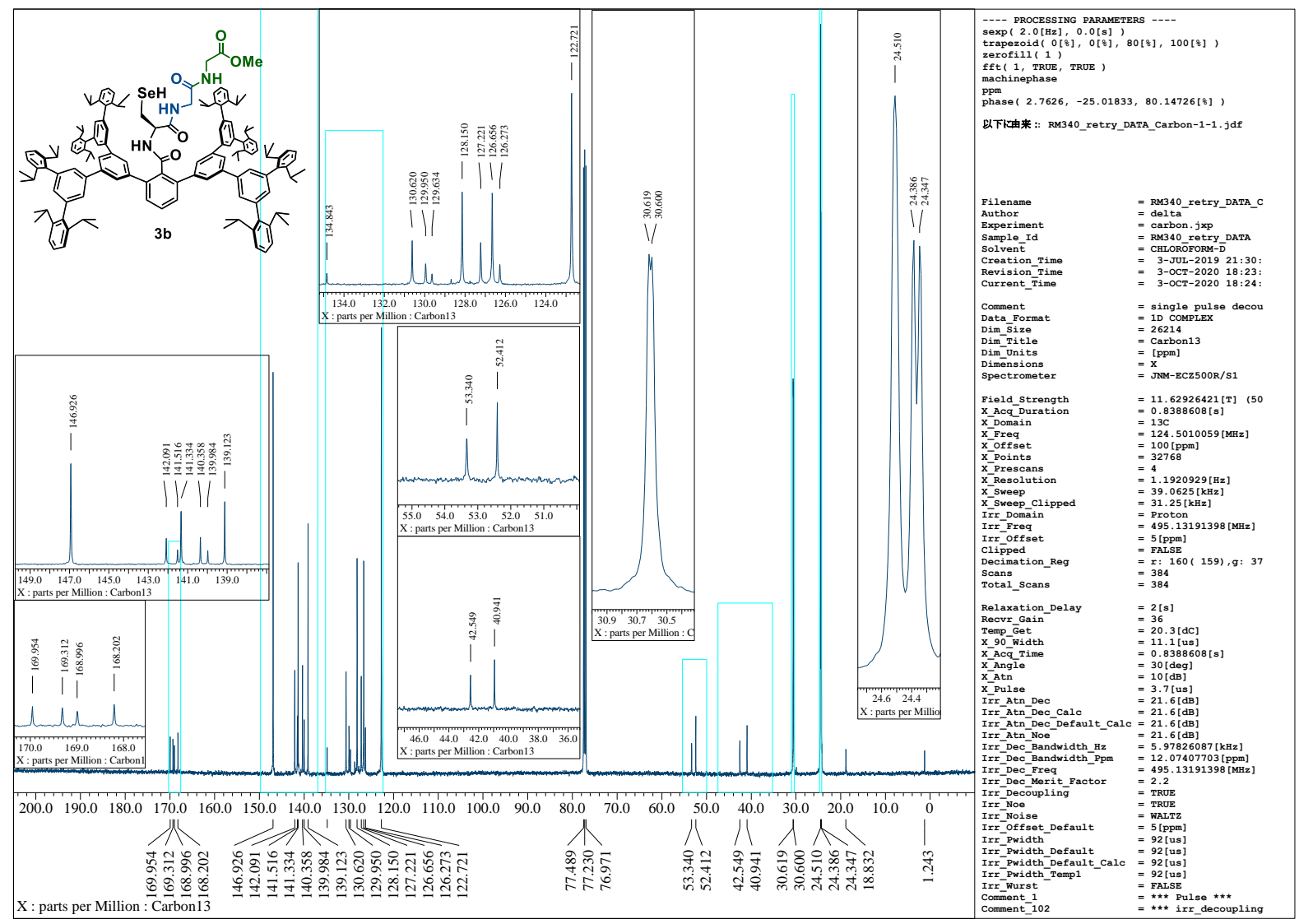

Figure $\mathbf{S 4 9} .{ }^{13} \mathrm{C}$ NMR $\left(125 \mathrm{MHz}, \mathrm{CDCl}_{3}\right)$ spectrum of $\mathbf{3 b}$.

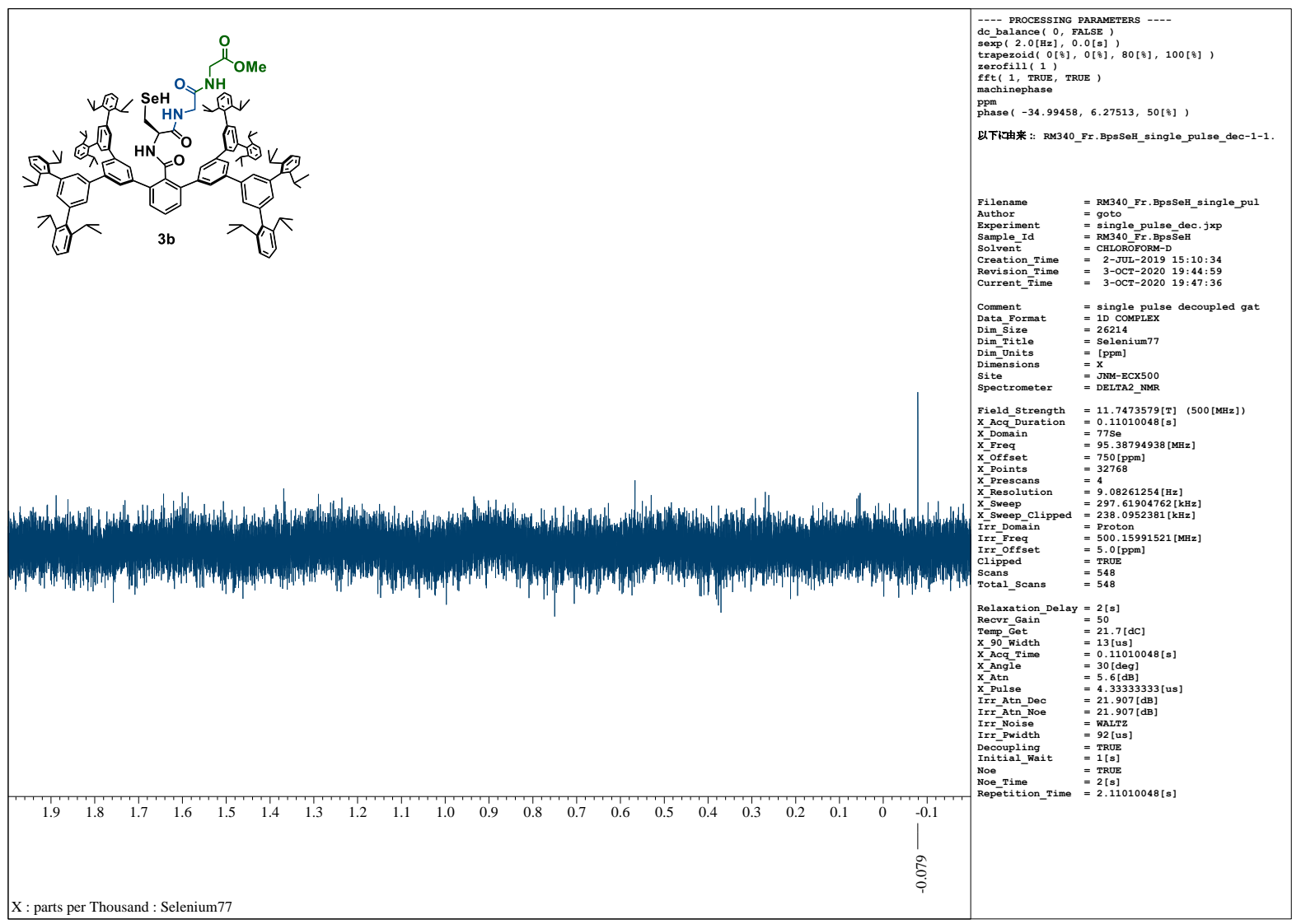

Figure S50. ${ }^{77} \mathrm{Se} \mathrm{NMR}\left(95 \mathrm{MHz}, \mathrm{CDCl}_{3}\right)$ spectrum of $\mathbf{3 b}$. 


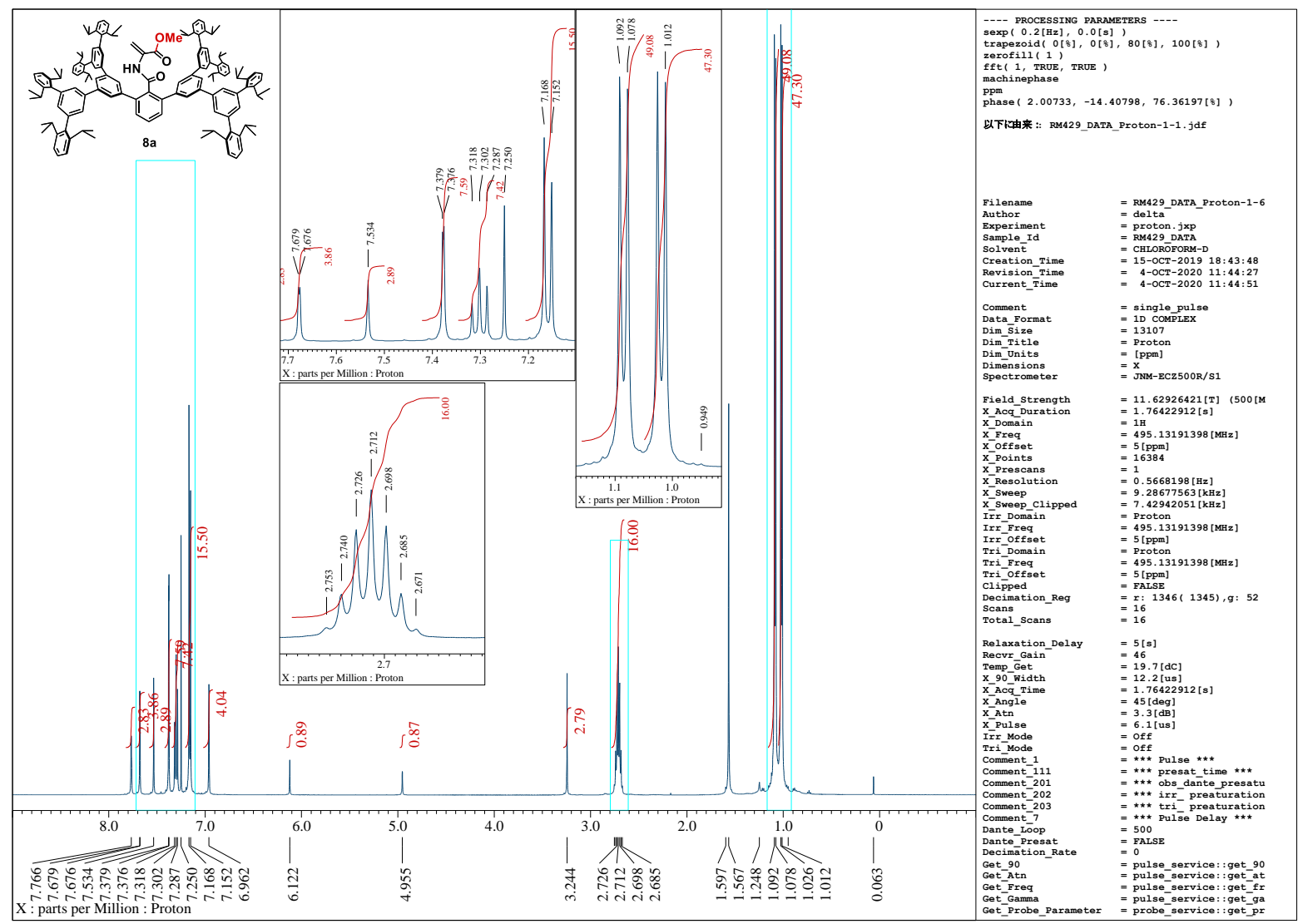

Figure S51. ${ }^{1} \mathrm{H}$ NMR $\left(500 \mathrm{MHz}, \mathrm{CDCl}_{3}\right)$ spectrum of $8 \mathbf{a}$.

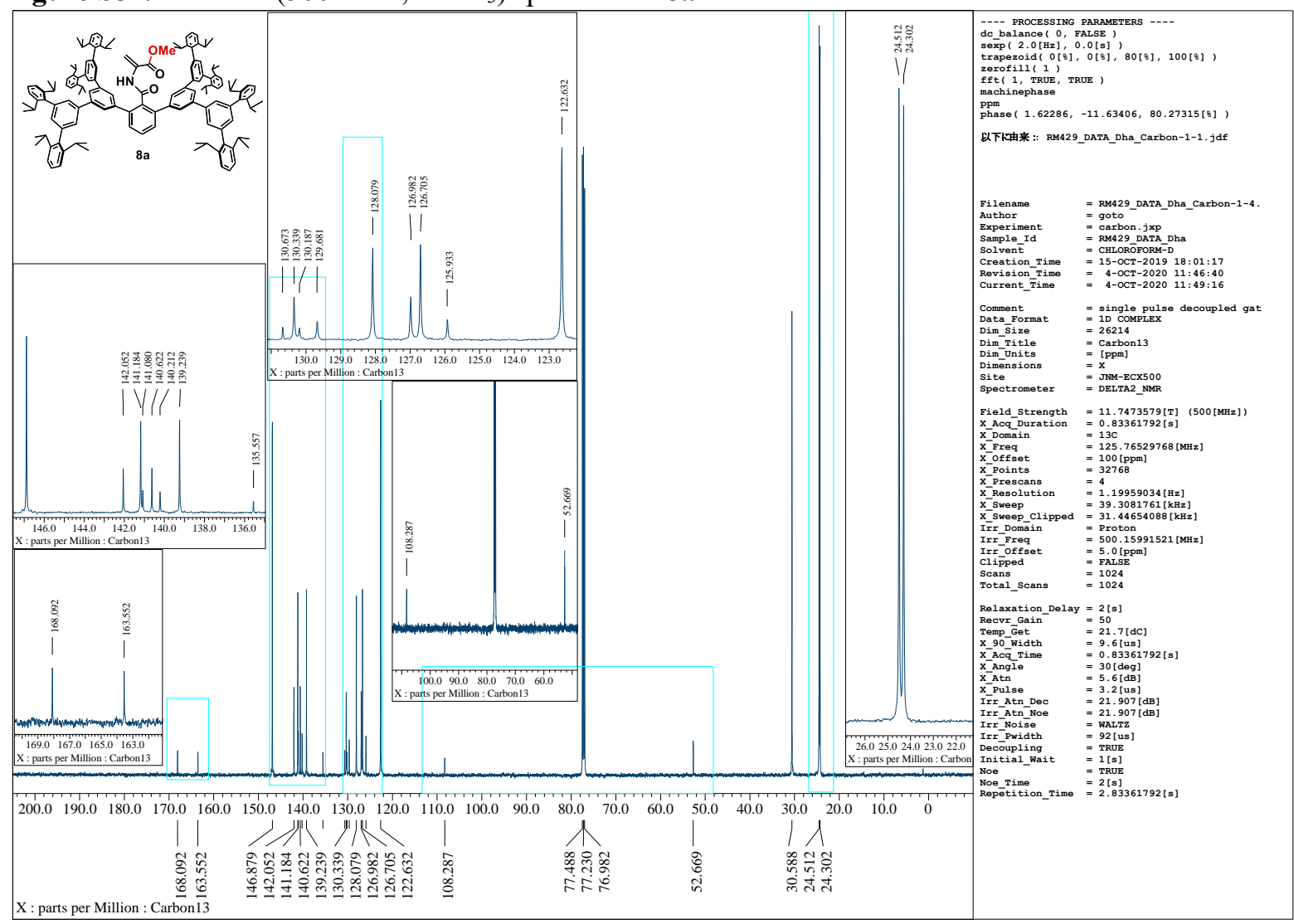

Figure S52. ${ }^{13} \mathrm{C}$ NMR $\left(125 \mathrm{MHz}, \mathrm{CDCl}_{3}\right)$ spectrum of $8 \mathbf{a}$. 


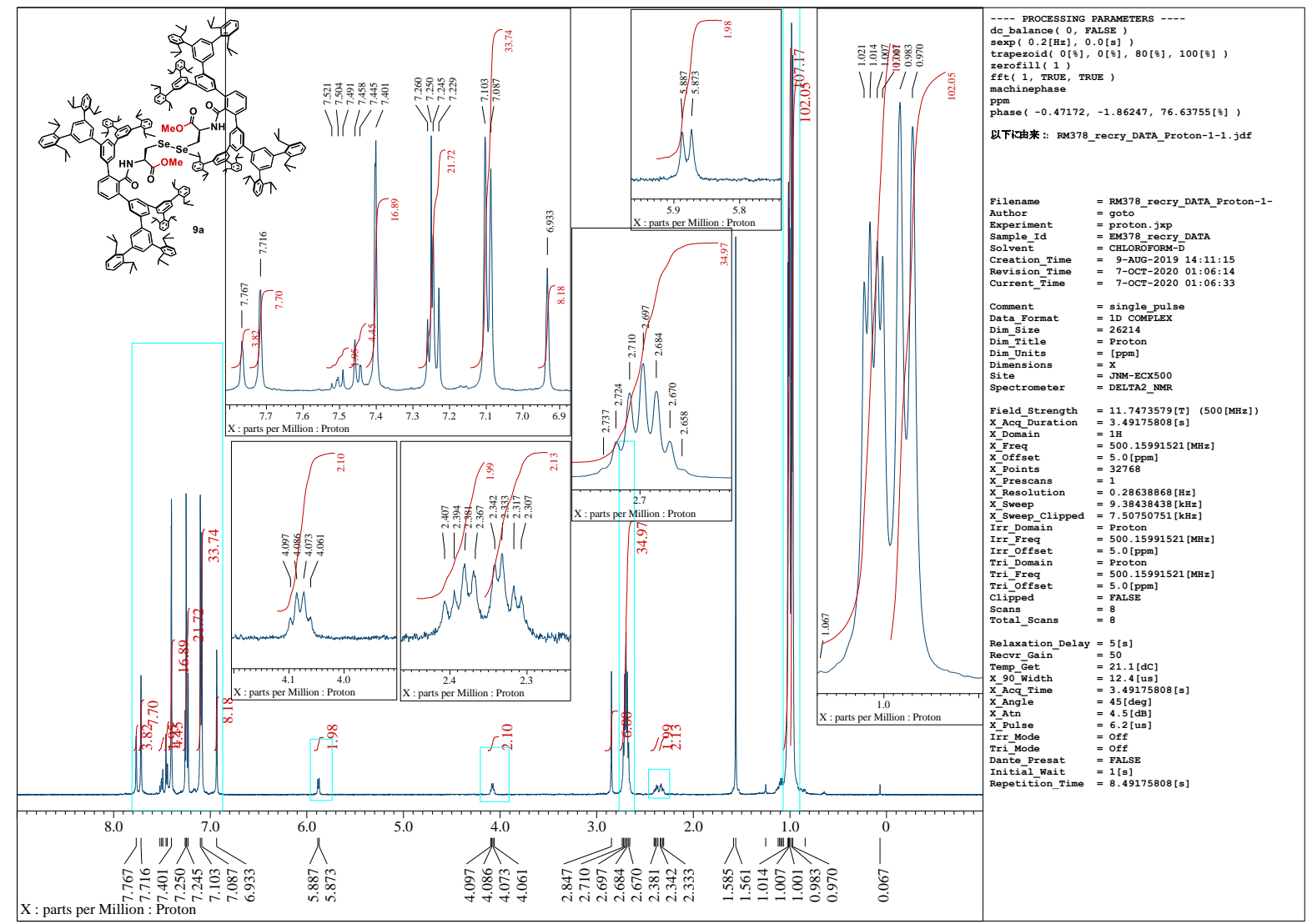

Figure S53. ${ }^{1} \mathrm{H}$ NMR $\left(500 \mathrm{MHz}, \mathrm{CDCl}_{3}\right)$ spectrum of 9a.

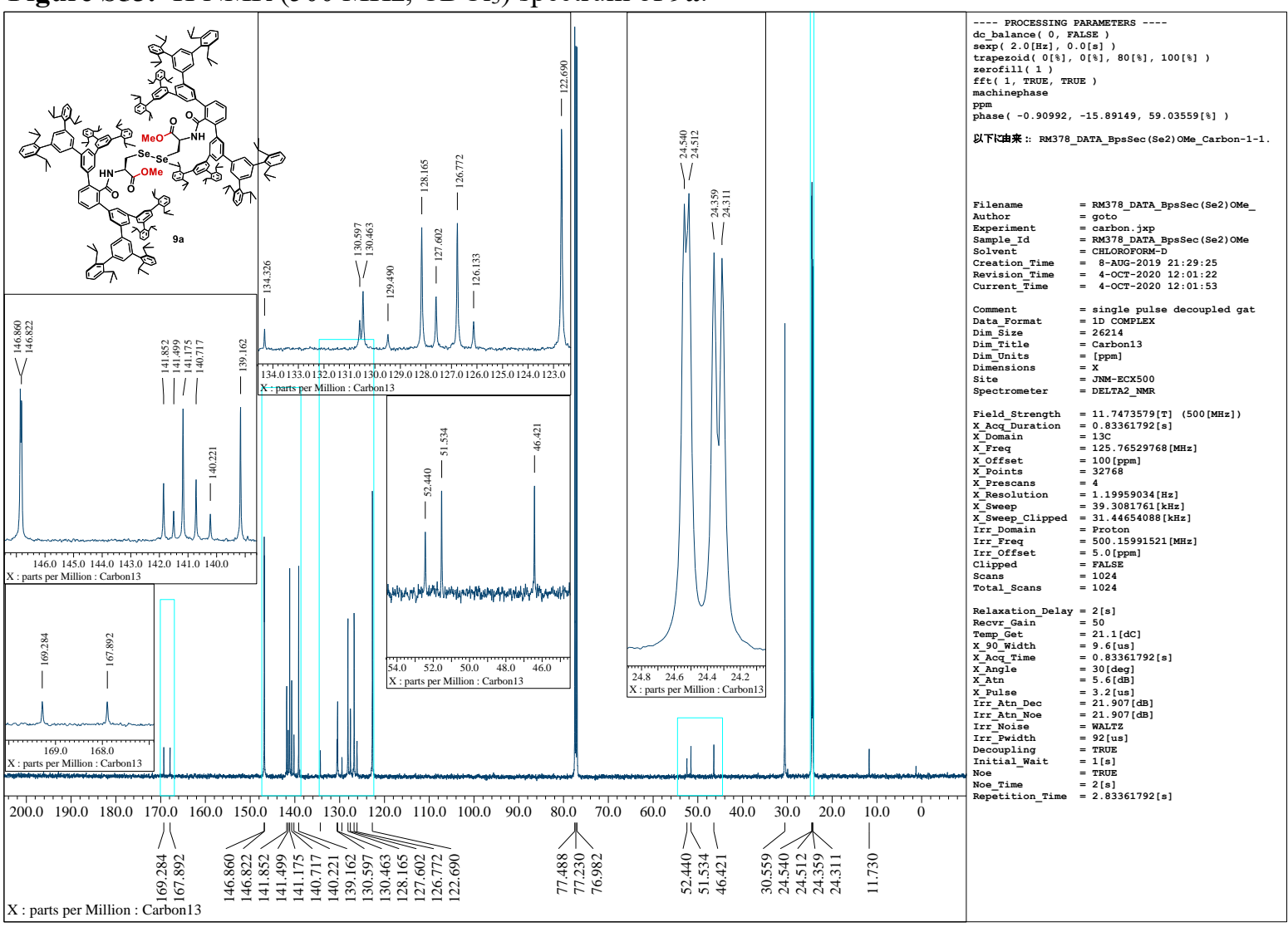

Figure S54. ${ }^{13} \mathrm{C}$ NMR $\left(125 \mathrm{MHz}, \mathrm{CDCl}_{3}\right)$ spectrum of 9a. 


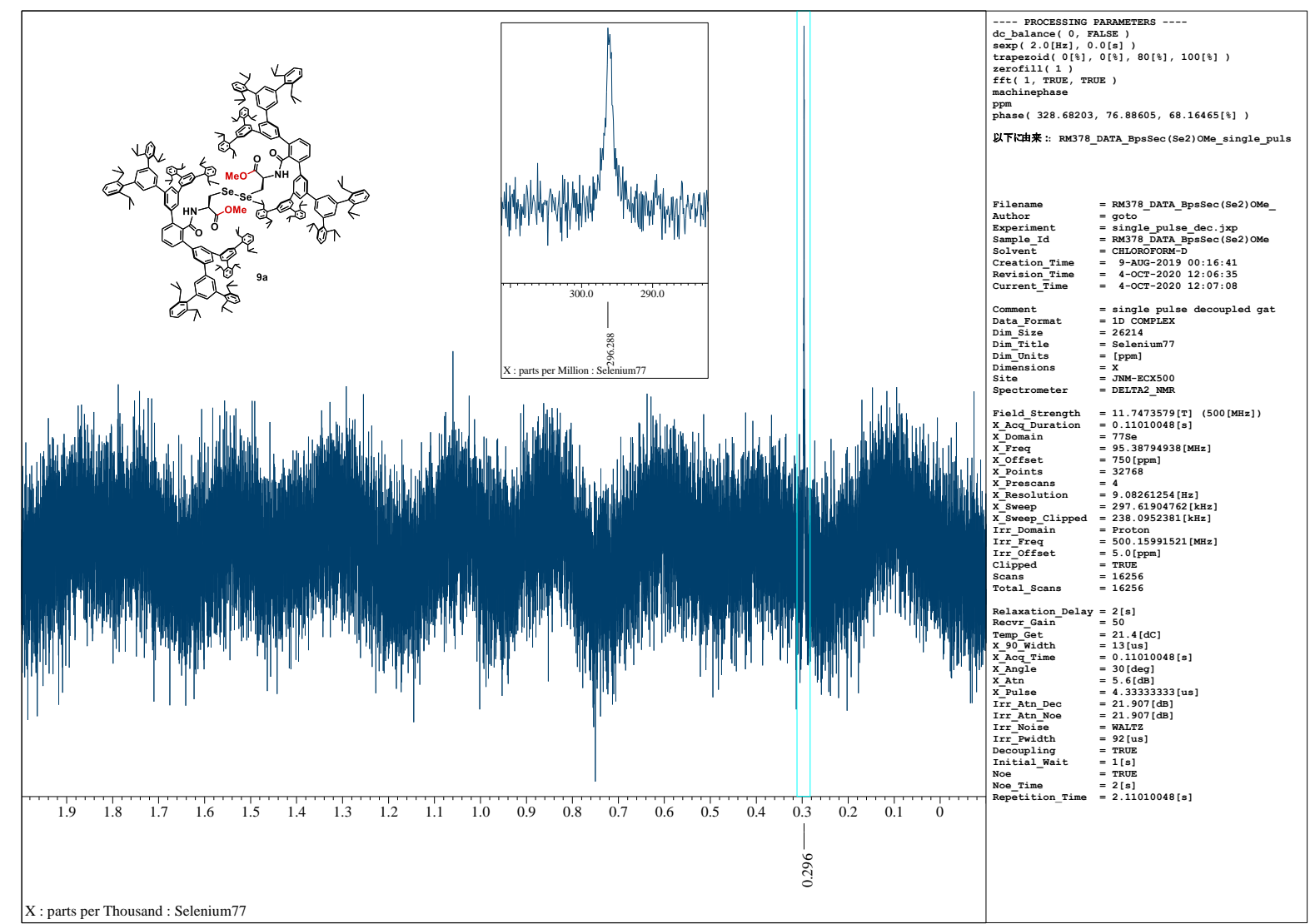

Figure S55. ${ }^{77} \mathrm{Se} \mathrm{NMR}\left(95 \mathrm{MHz}, \mathrm{CDCl}_{3}\right)$ spectrum of $9 \mathbf{a}$.

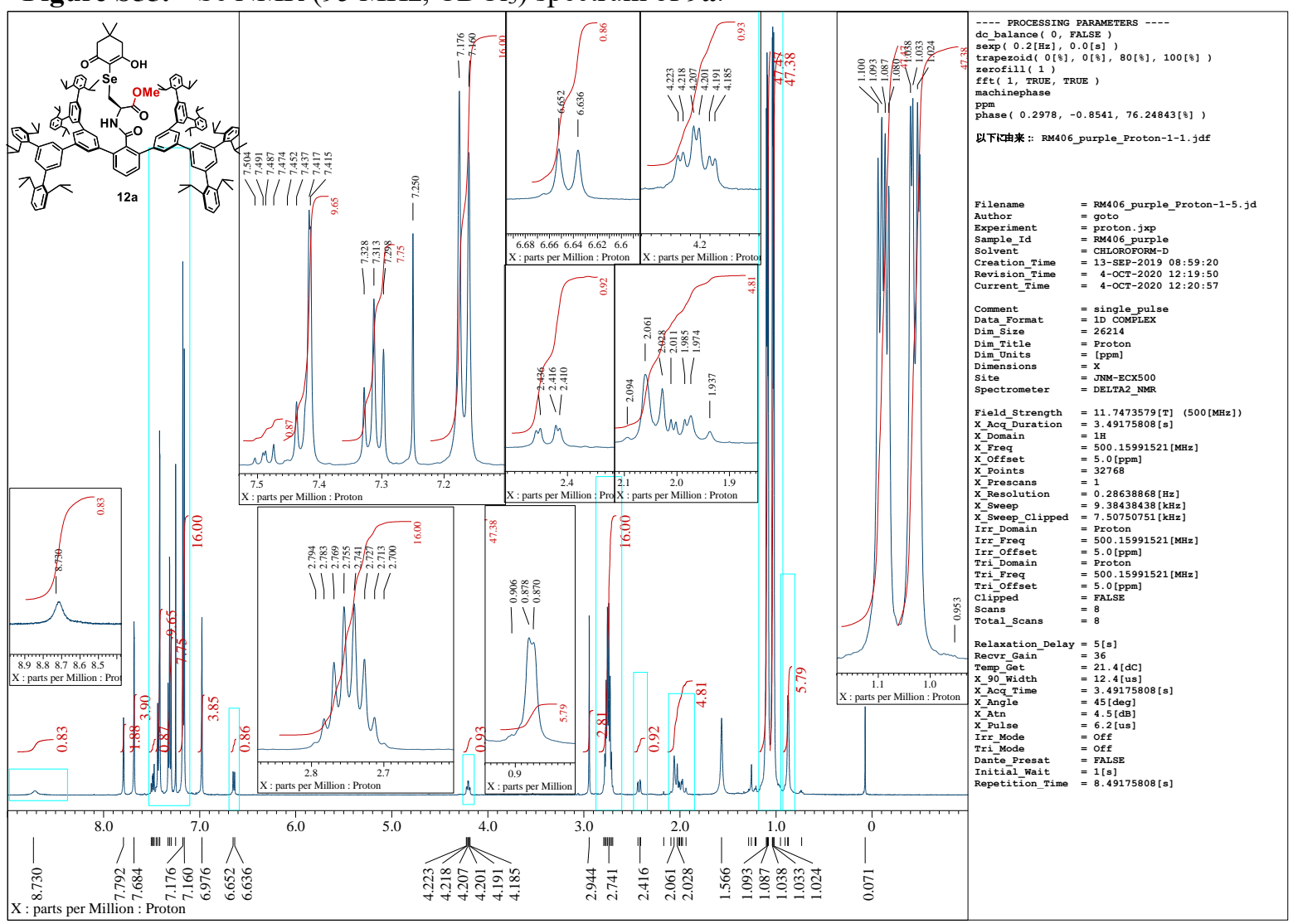

Figure S56. ${ }^{1} \mathrm{H}$ NMR $\left(500 \mathrm{MHz}, \mathrm{CDCl}_{3}\right)$ spectrum of 12a. 


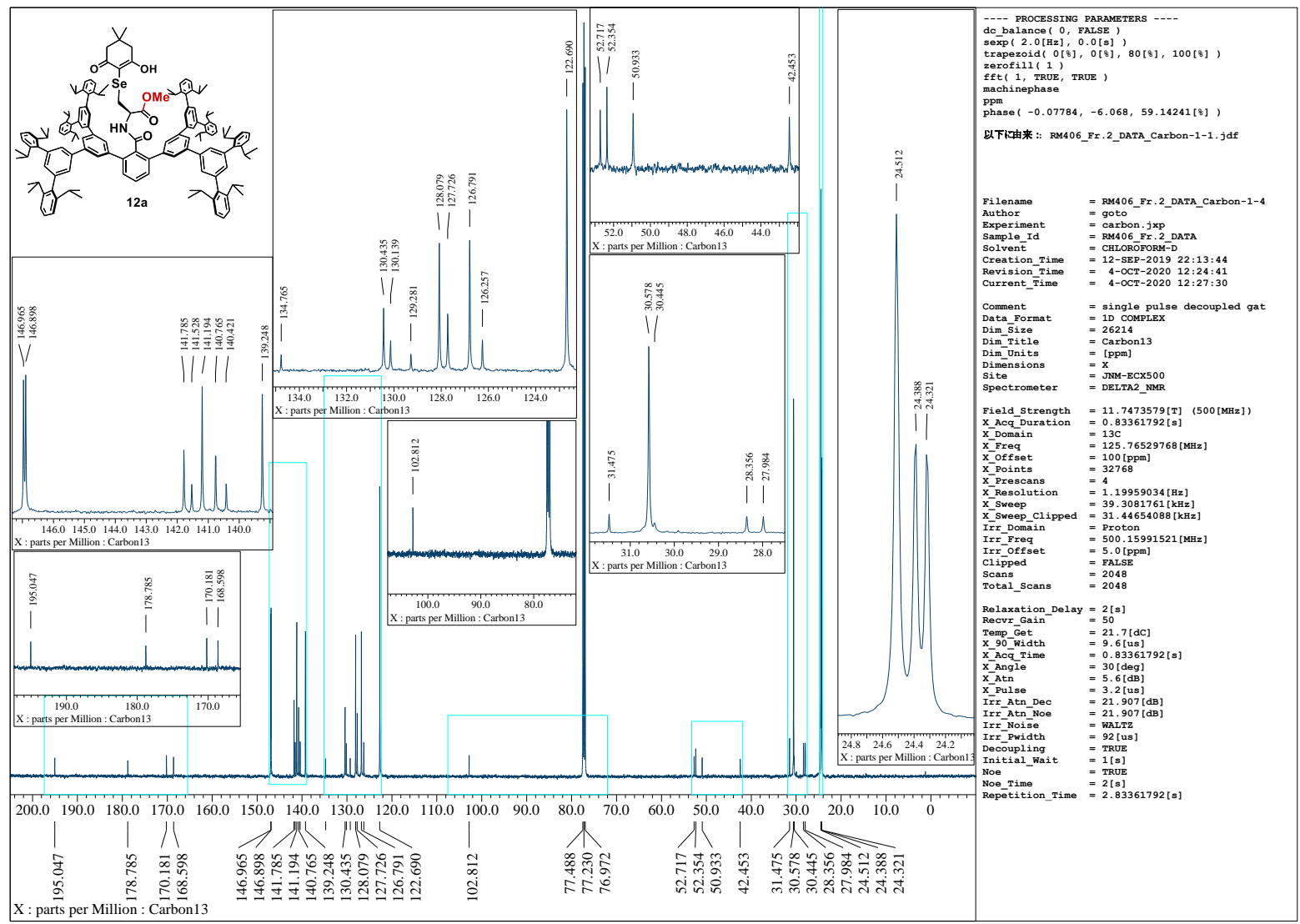

Figure S57. ${ }^{13} \mathrm{C}$ NMR $\left(125 \mathrm{MHz}, \mathrm{CDCl}_{3}\right)$ spectrum of 12a.

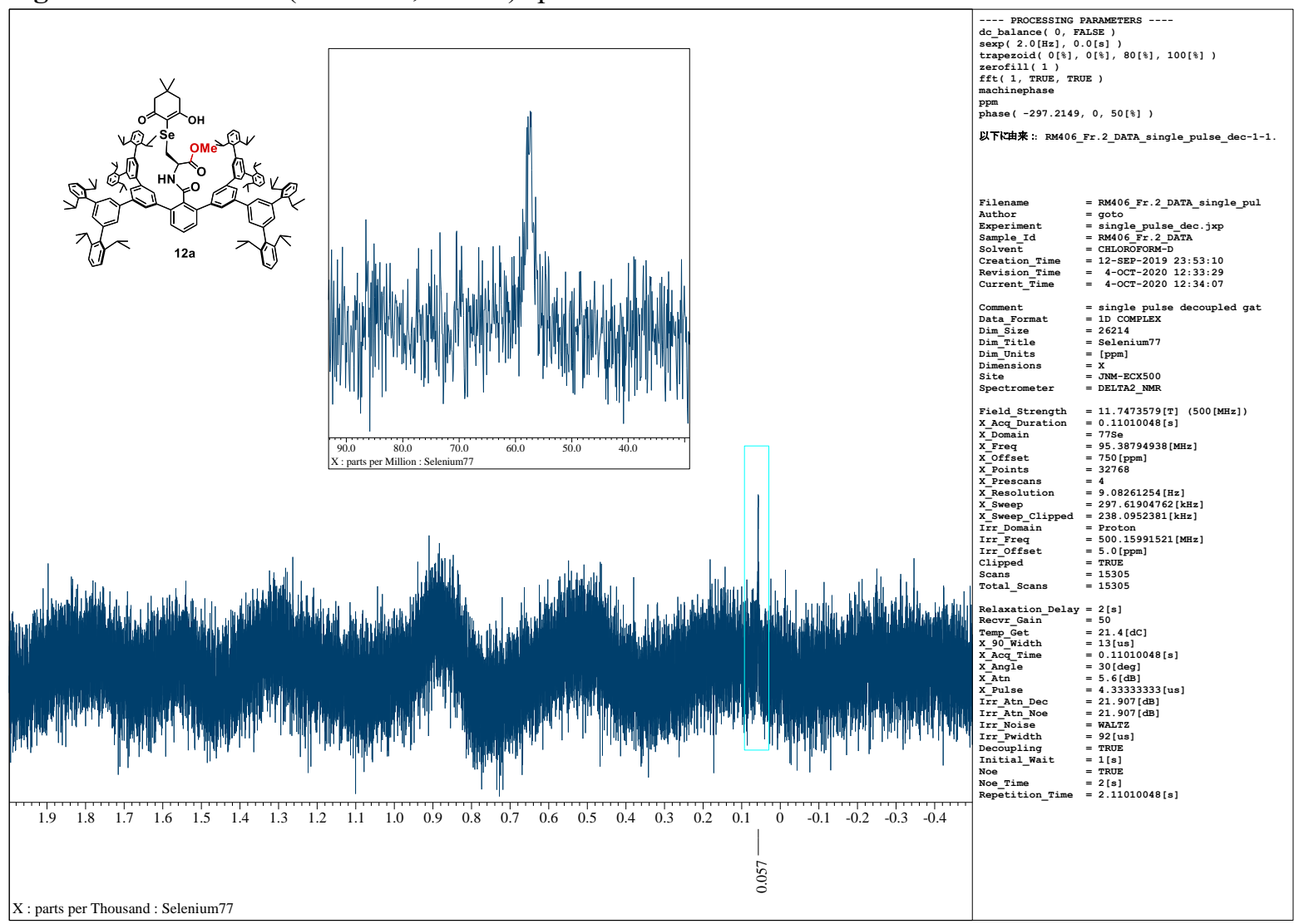

Figure S58. ${ }^{77} \mathrm{Se} \mathrm{NMR}\left(95 \mathrm{MHz}, \mathrm{CDCl}_{3}\right.$ ) spectrum of 12a. 


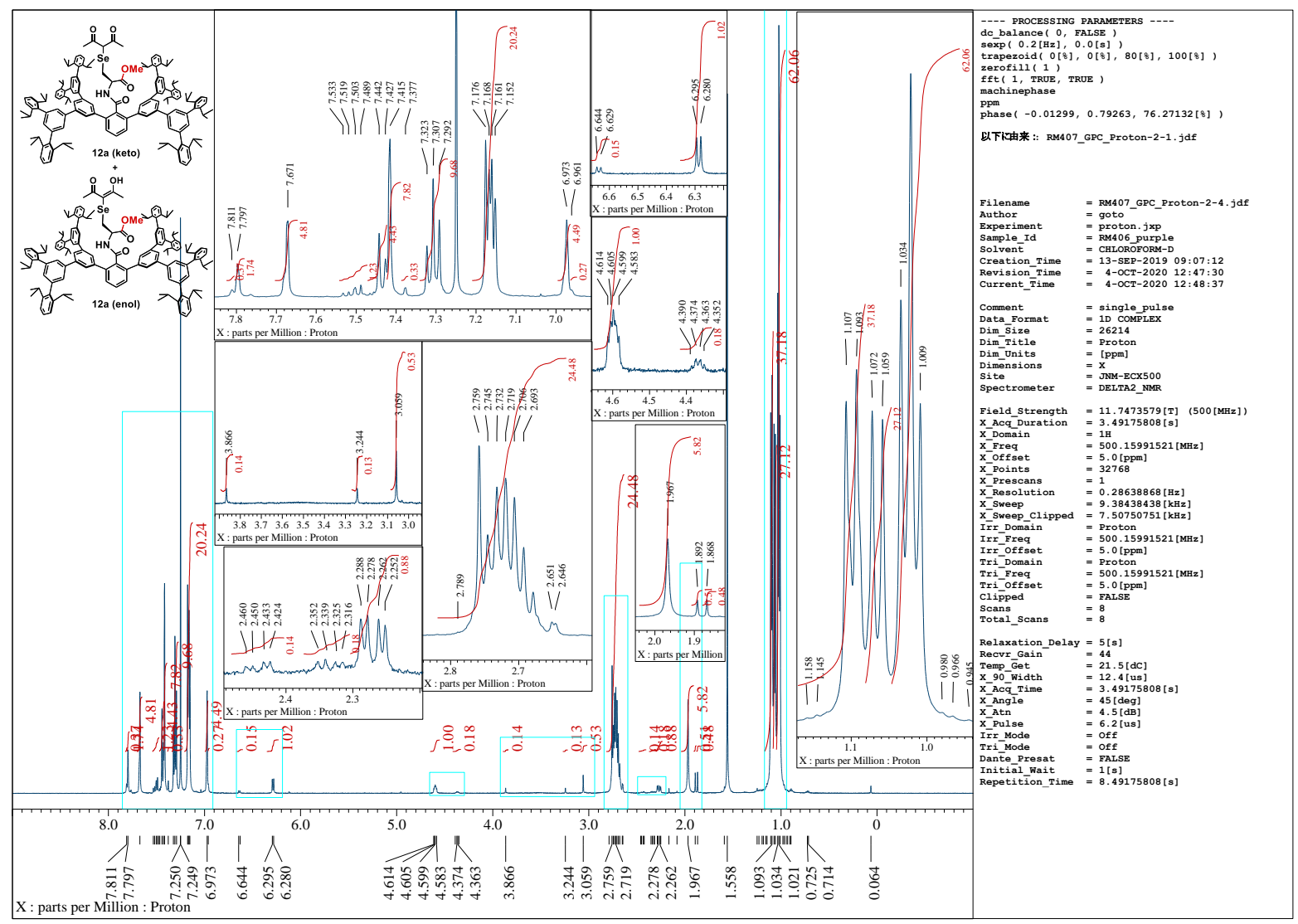

Figure S59. ${ }^{1} \mathrm{H}$ NMR $\left(500 \mathrm{MHz}, \mathrm{CDCl}_{3}\right)$ spectrum of tautomeric mixture of 13a (keto/enol = 15:85).

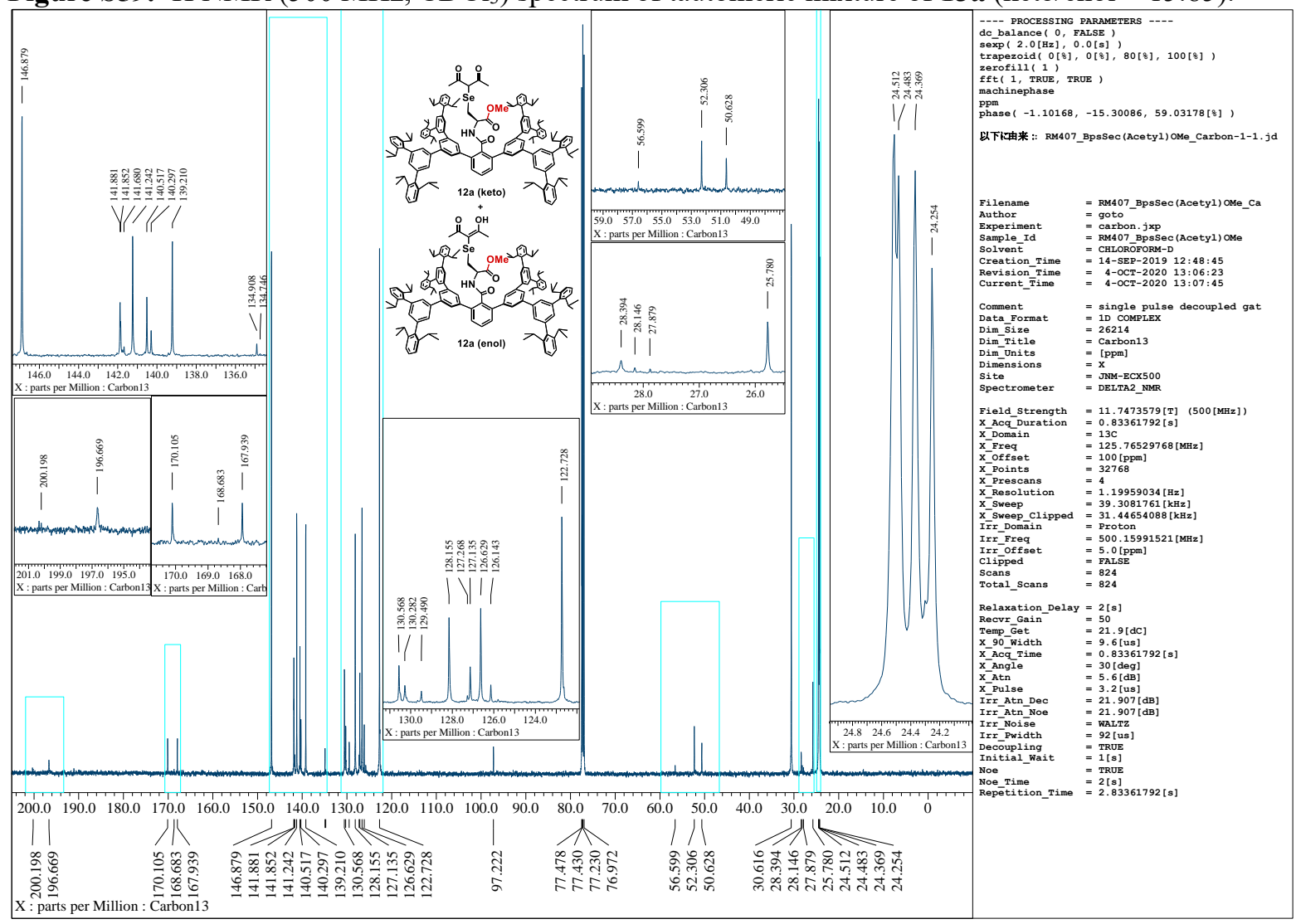

Figure S60. ${ }^{13} \mathrm{C}$ NMR $\left(125 \mathrm{MHz}, \mathrm{CDCl}_{3}\right)$ spectrum of tautomeric mixture of 13a (keto/enol = 15:85). 


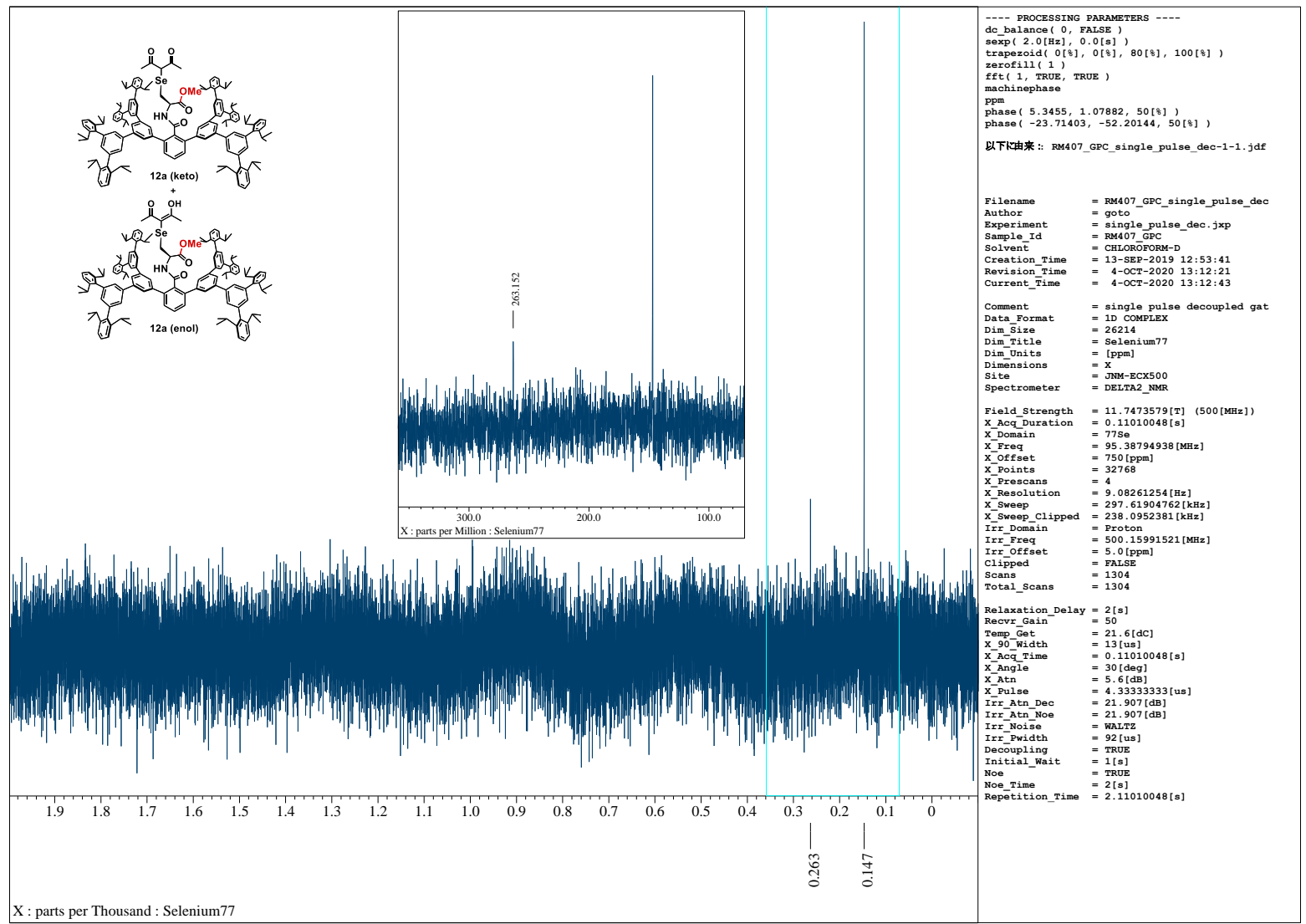

Figure S61. ${ }^{77} \mathrm{Se}$ NMR (95 MHz, $\left.\mathrm{CDCl}_{3}\right)$ spectrum of tautomeric mixture of 13a (keto/enol = 15:85).

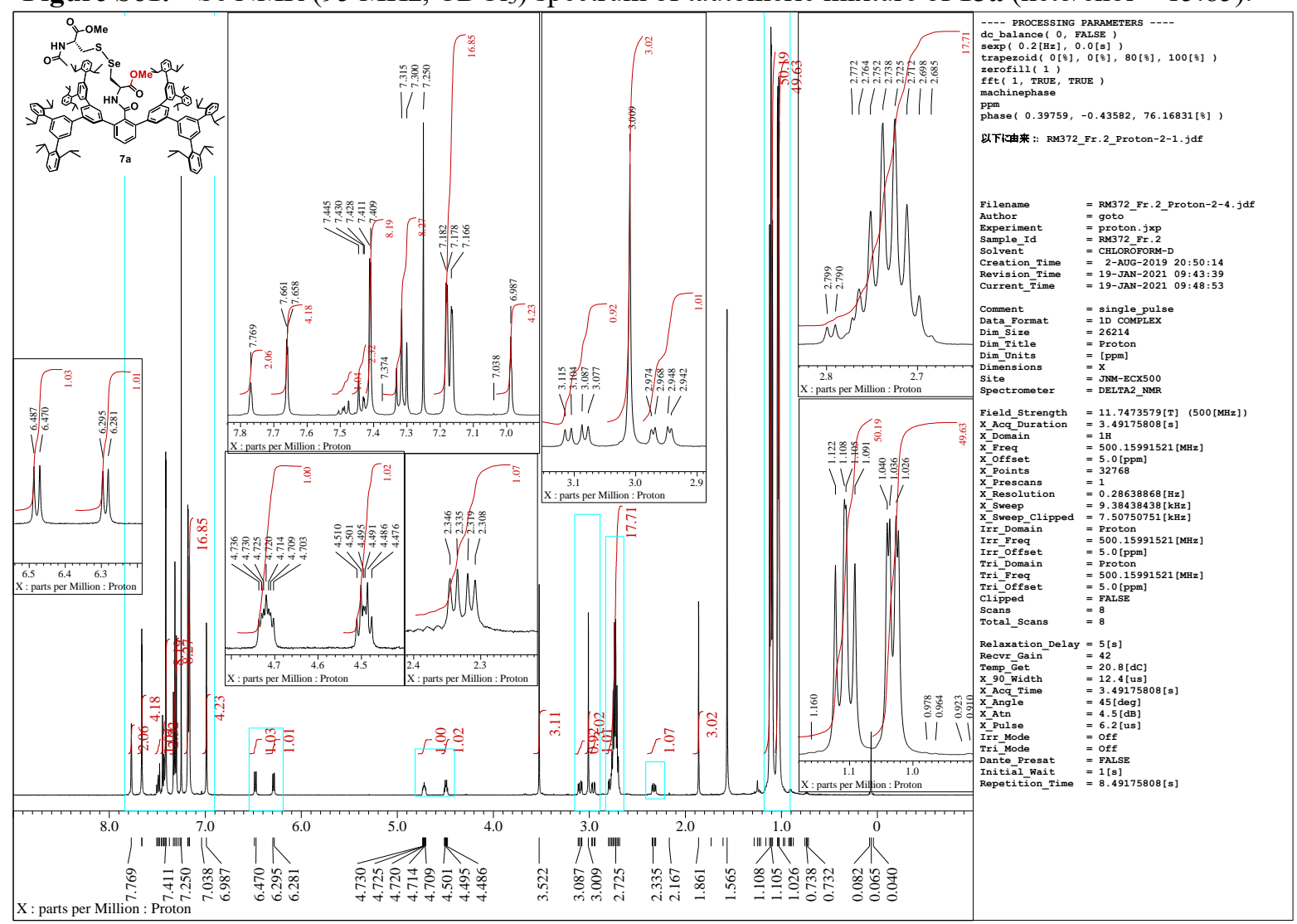

Figure $\mathbf{S 6 2} .{ }^{1} \mathrm{H}$ NMR $\left(500 \mathrm{MHz}, \mathrm{CDCl}_{3}\right)$ spectrum of $7 \mathbf{a}$. 


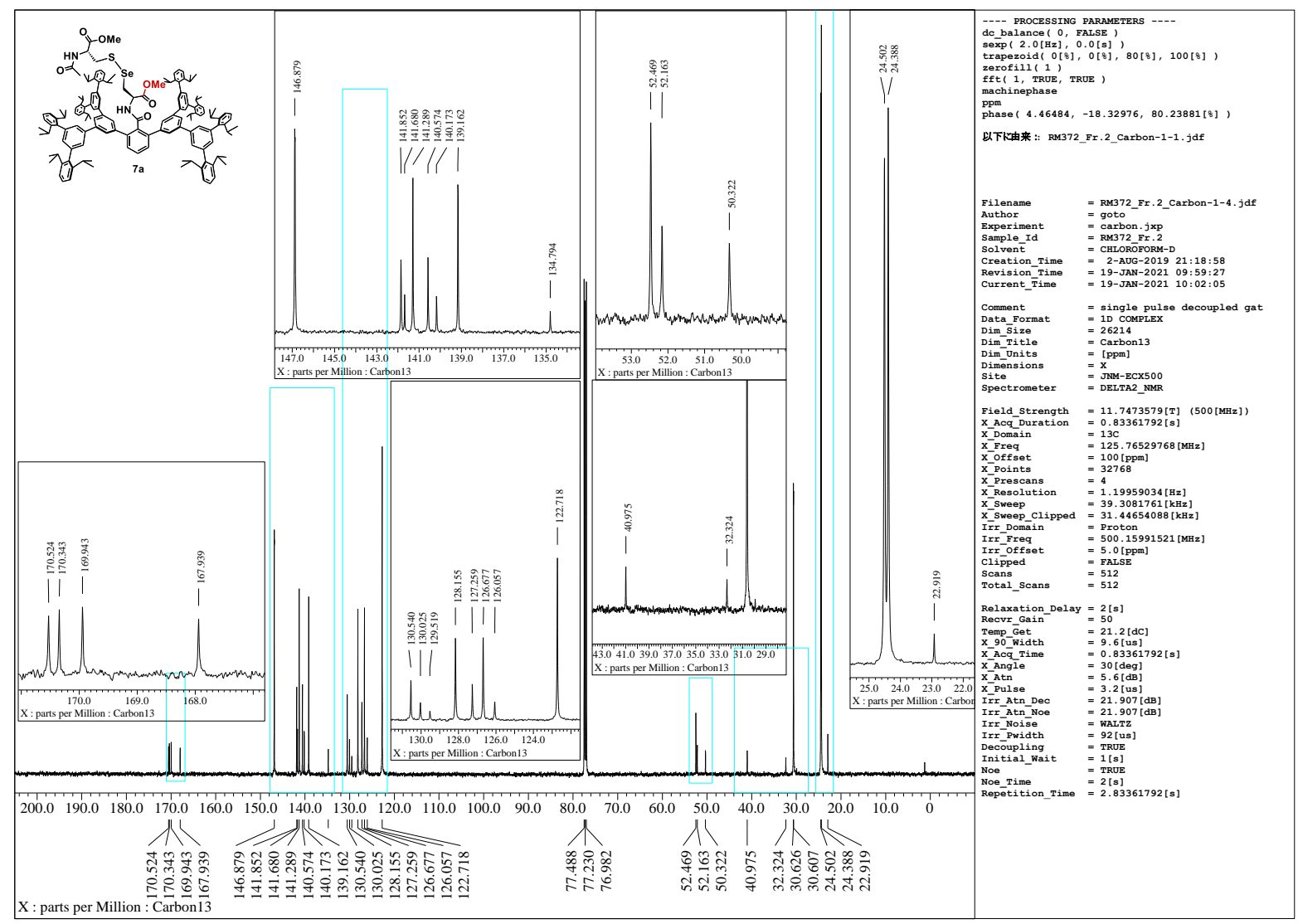

Figure S63. ${ }^{13} \mathrm{C}$ NMR $\left(125 \mathrm{MHz}, \mathrm{CDCl}_{3}\right)$ spectrum of $7 \mathbf{a}$.

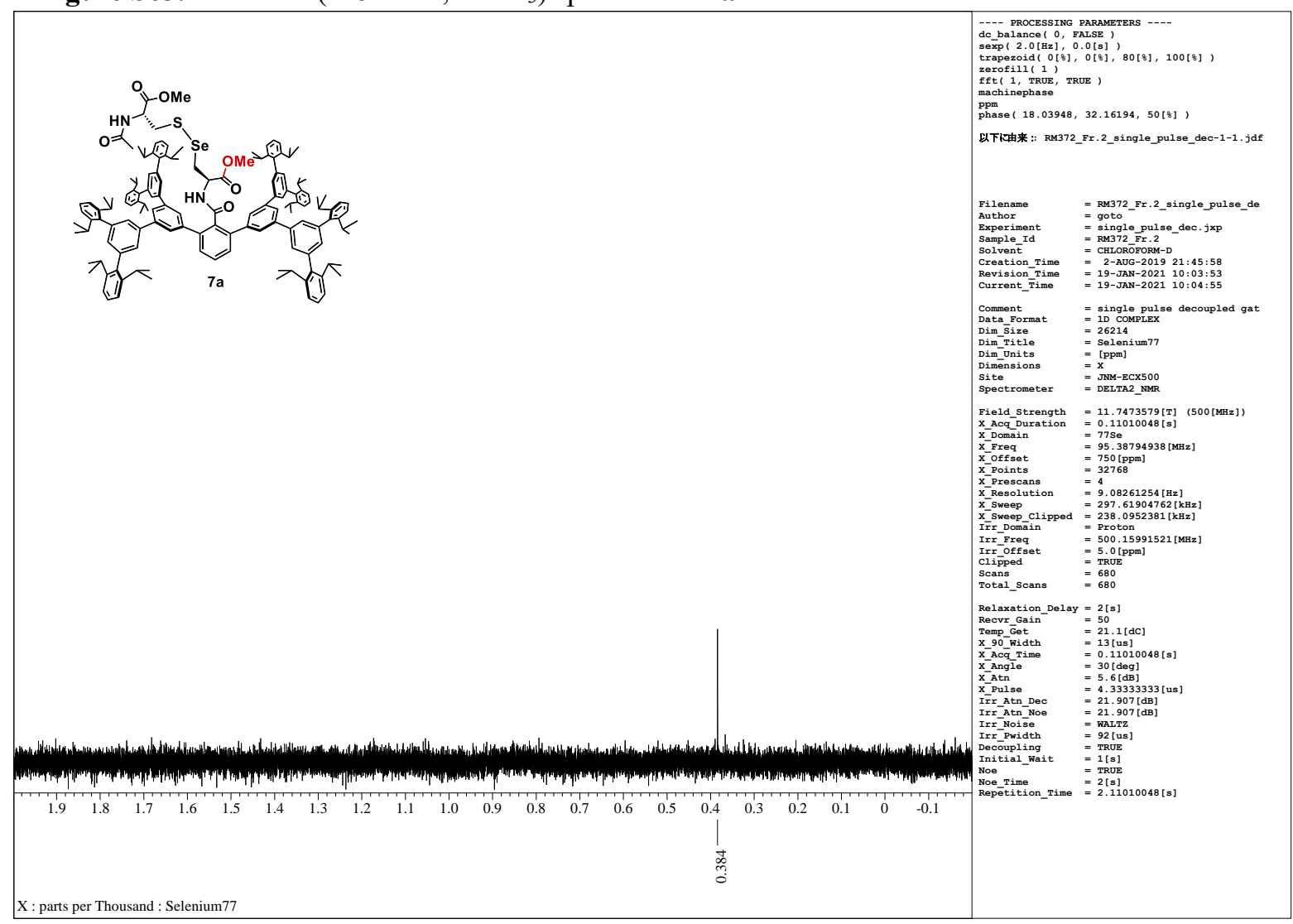

Figure S64. ${ }^{77} \mathrm{Se} \mathrm{NMR}\left(95 \mathrm{MHz}, \mathrm{CDCl}_{3}\right)$ spectrum of $\mathbf{7 a}$. 


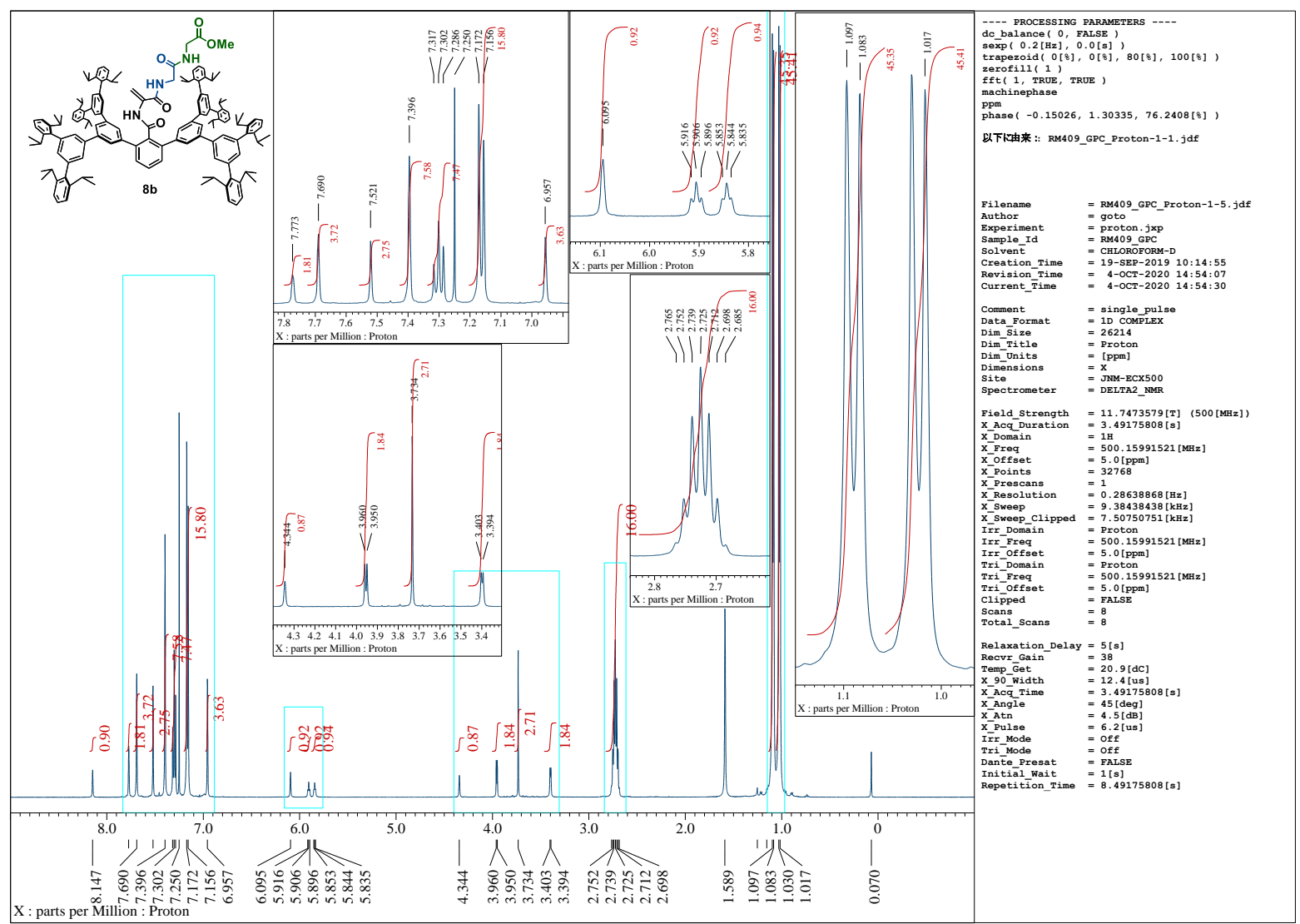

Figure S65. ${ }^{1} \mathrm{H}$ NMR $\left(500 \mathrm{MHz}, \mathrm{CDCl}_{3}\right)$ spectrum of $\mathbf{8 b}$.

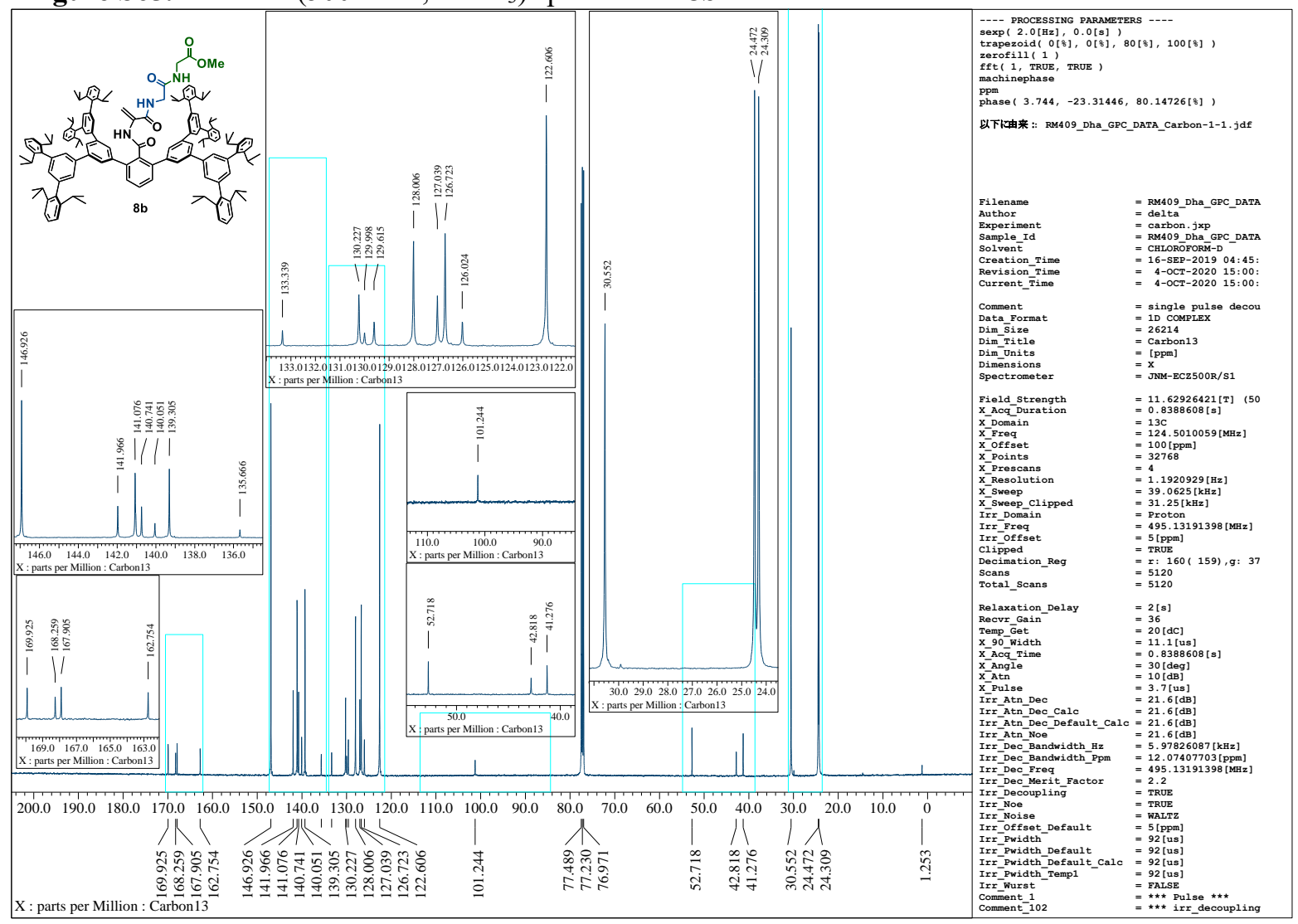

Figure S66. ${ }^{13} \mathrm{C}$ NMR $\left(125 \mathrm{MHz}, \mathrm{CDCl}_{3}\right)$ spectrum of $\mathbf{8 b}$. 


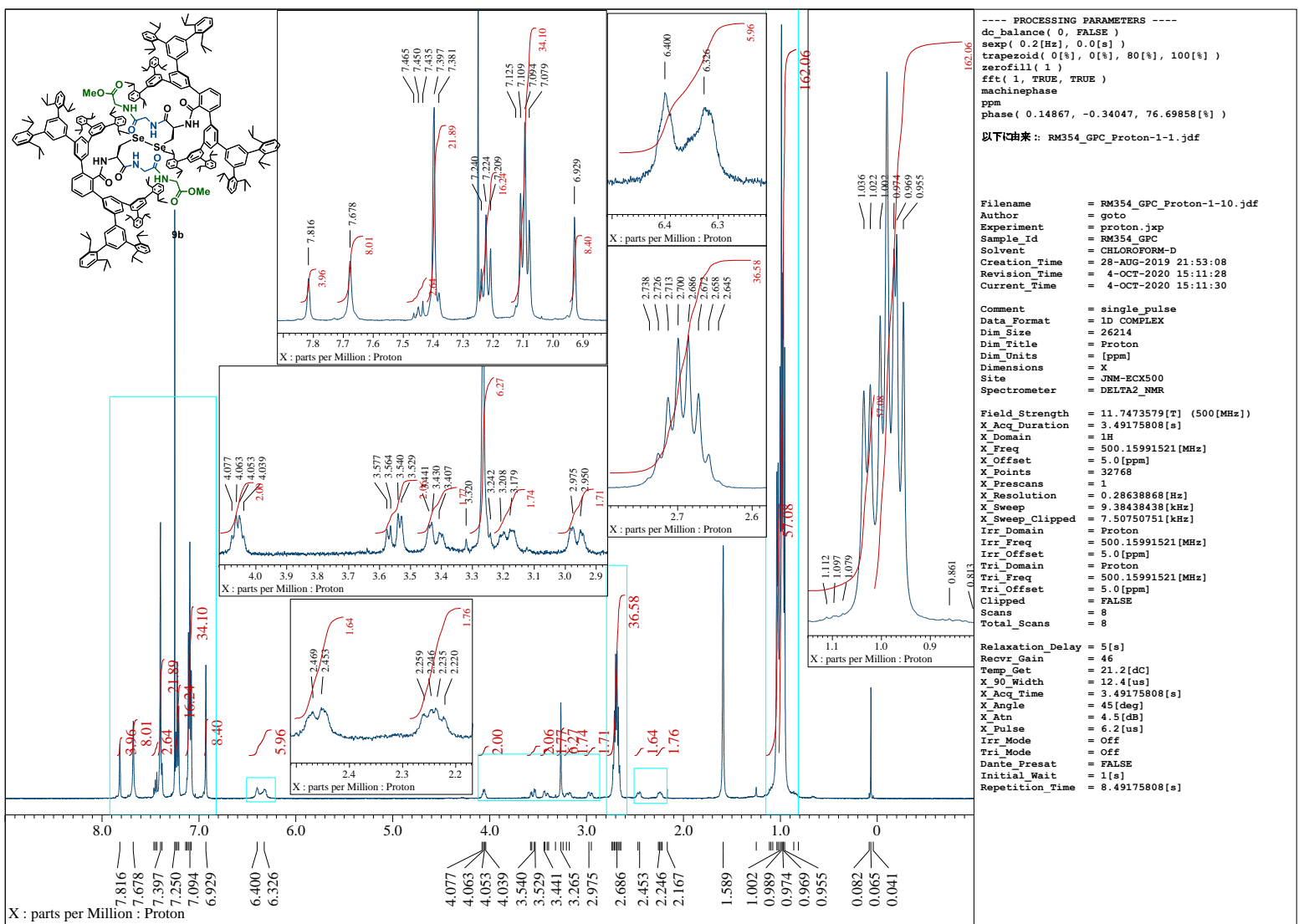

Figure S67. ${ }^{1} \mathrm{H}$ NMR (500 MHz, $\left.\mathrm{CDCl}_{3}\right)$ spectrum of $9 \mathbf{b}$.

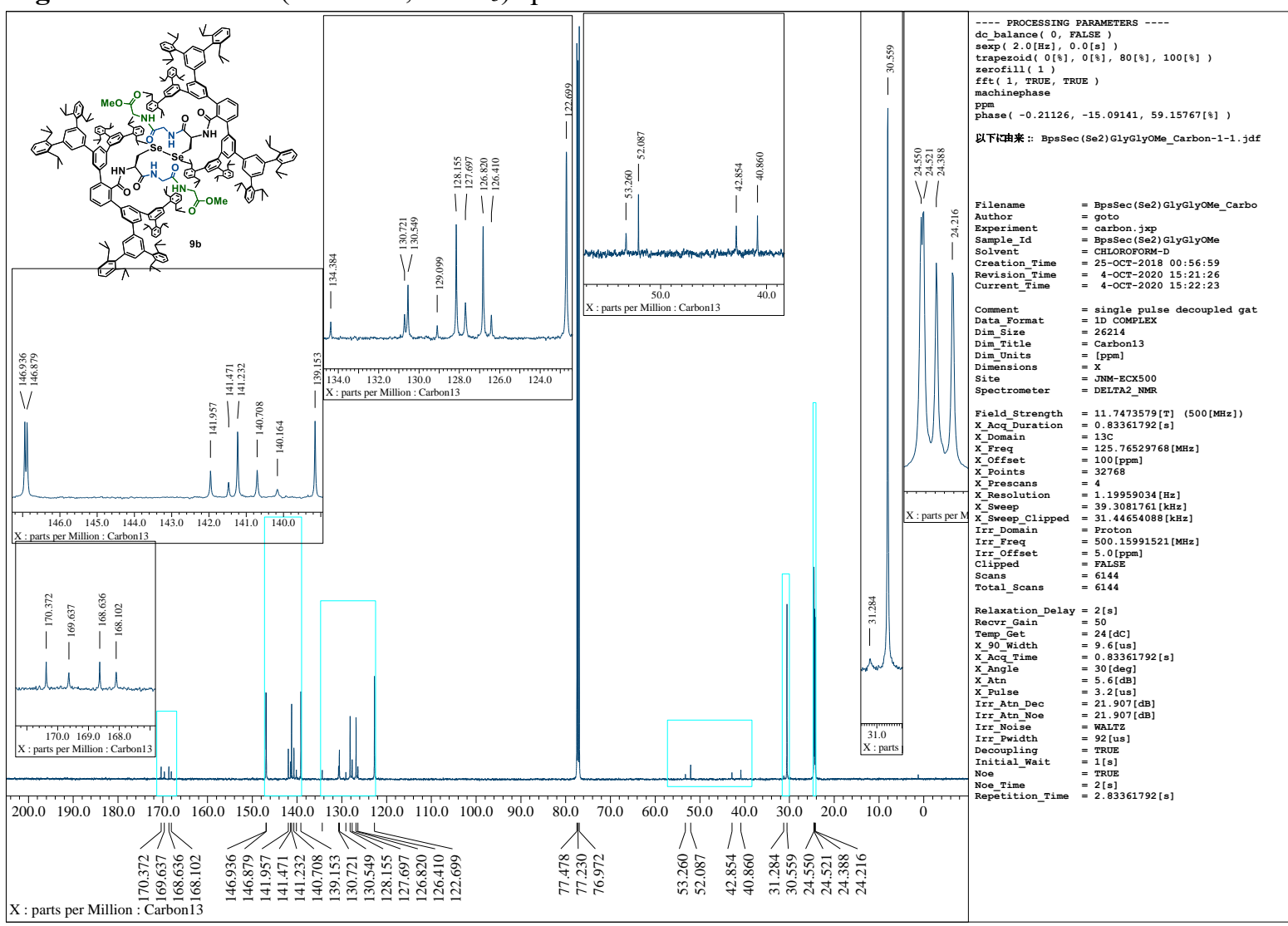

Figure S68. ${ }^{13} \mathrm{C}$ NMR $\left(125 \mathrm{MHz}, \mathrm{CDCl}_{3}\right)$ spectrum of $\mathbf{9 b}$. 


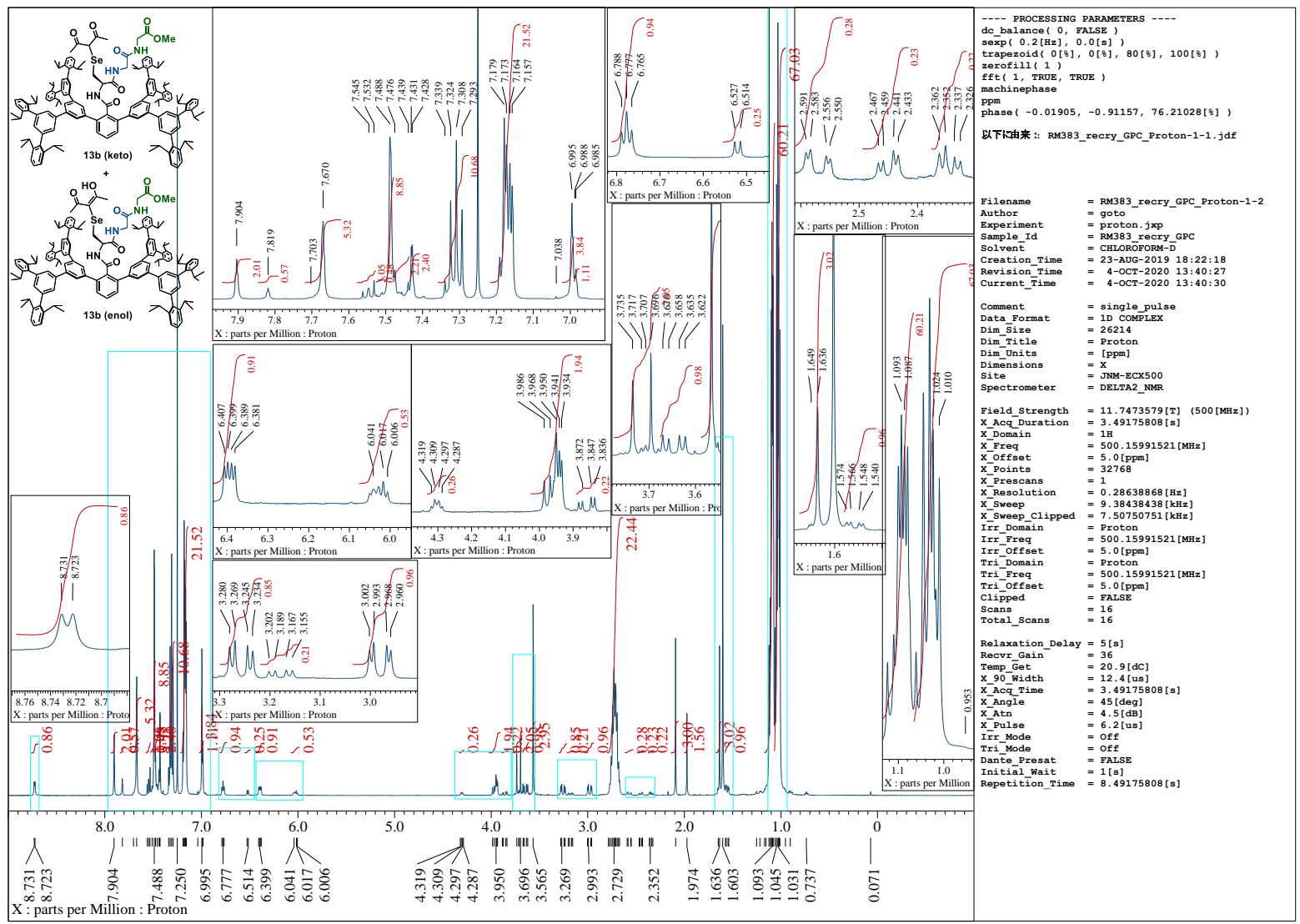

Figure S69. ${ }^{1} \mathrm{H}$ NMR $\left(500 \mathrm{MHz}, \mathrm{CDCl}_{3}\right)$ spectrum of tautomeric mixture of $\mathbf{1 3 b}$ (keto/enol = 66:34).

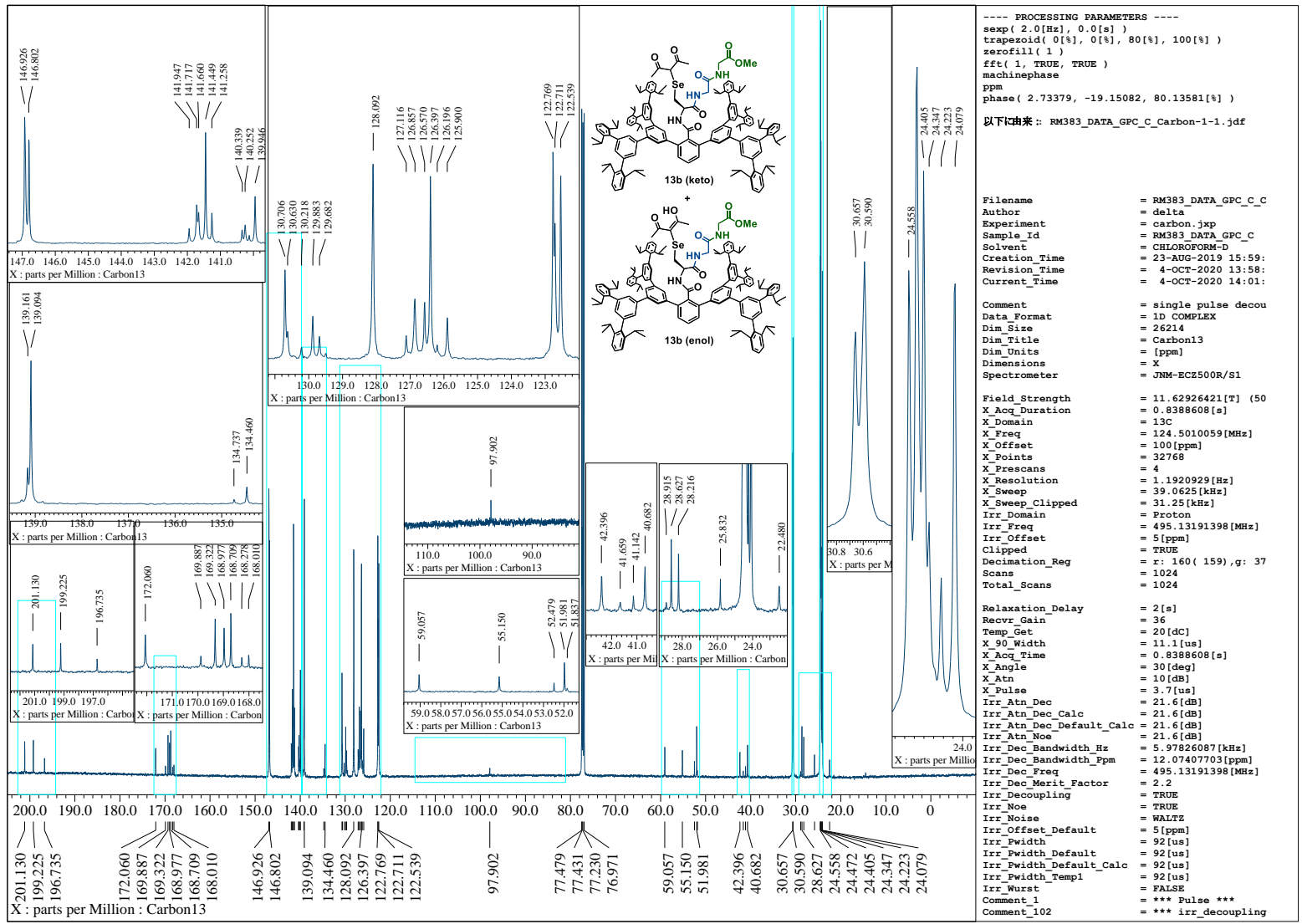

Figure S70. ${ }^{13} \mathrm{C}$ NMR $\left(125 \mathrm{MHz}, \mathrm{CDCl}_{3}\right)$ spectrum of tautomeric mixture of $\mathbf{1 3 b}$ (keto/enol = 66:34). 


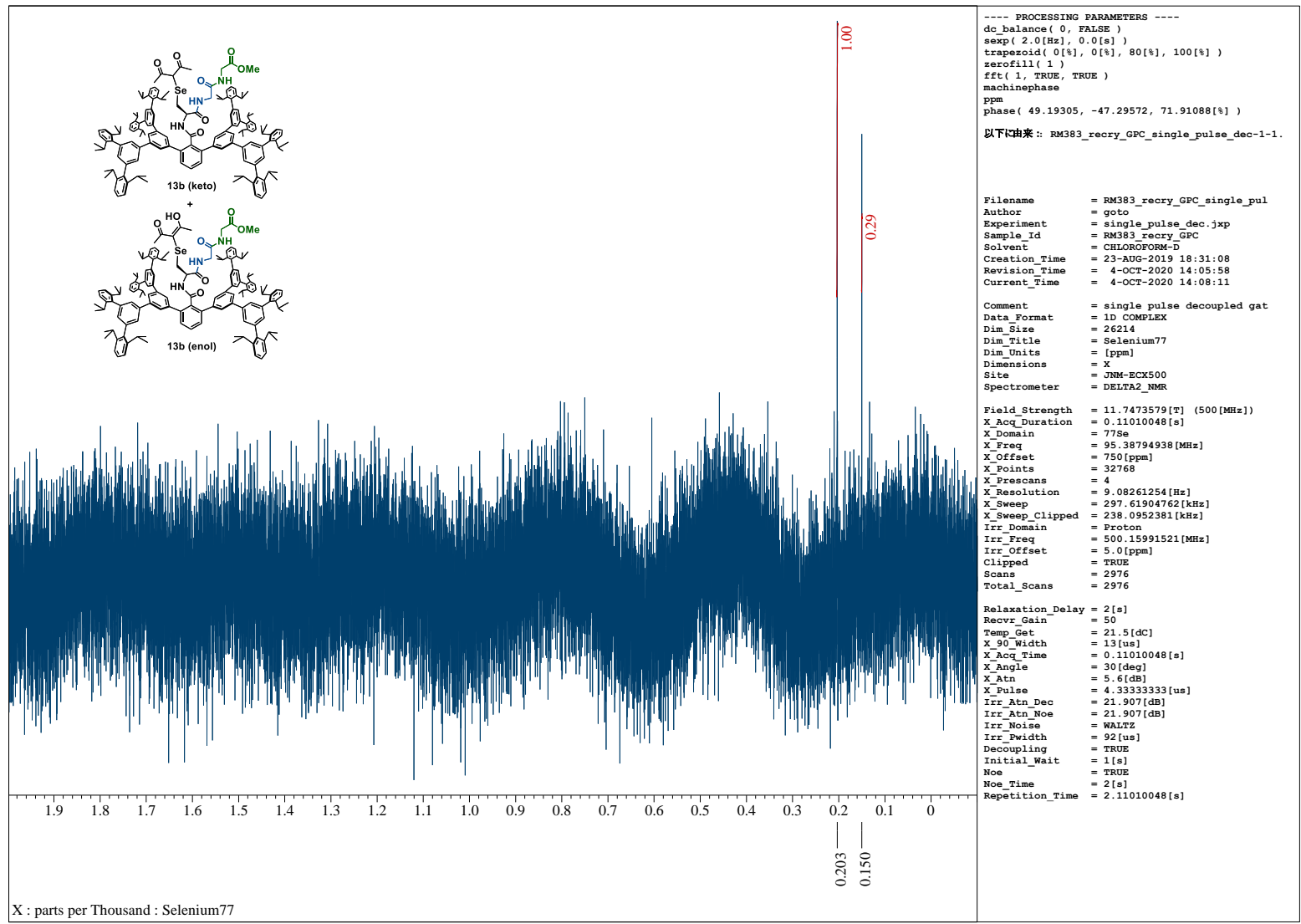

Figure S71. ${ }^{77} \mathrm{Se} \mathrm{NMR}\left(95 \mathrm{MHz}, \mathrm{CDCl}_{3}\right)$ spectrum of tautomeric mixture of $\mathbf{1 3 b}$ (keto/enol = 78:22).

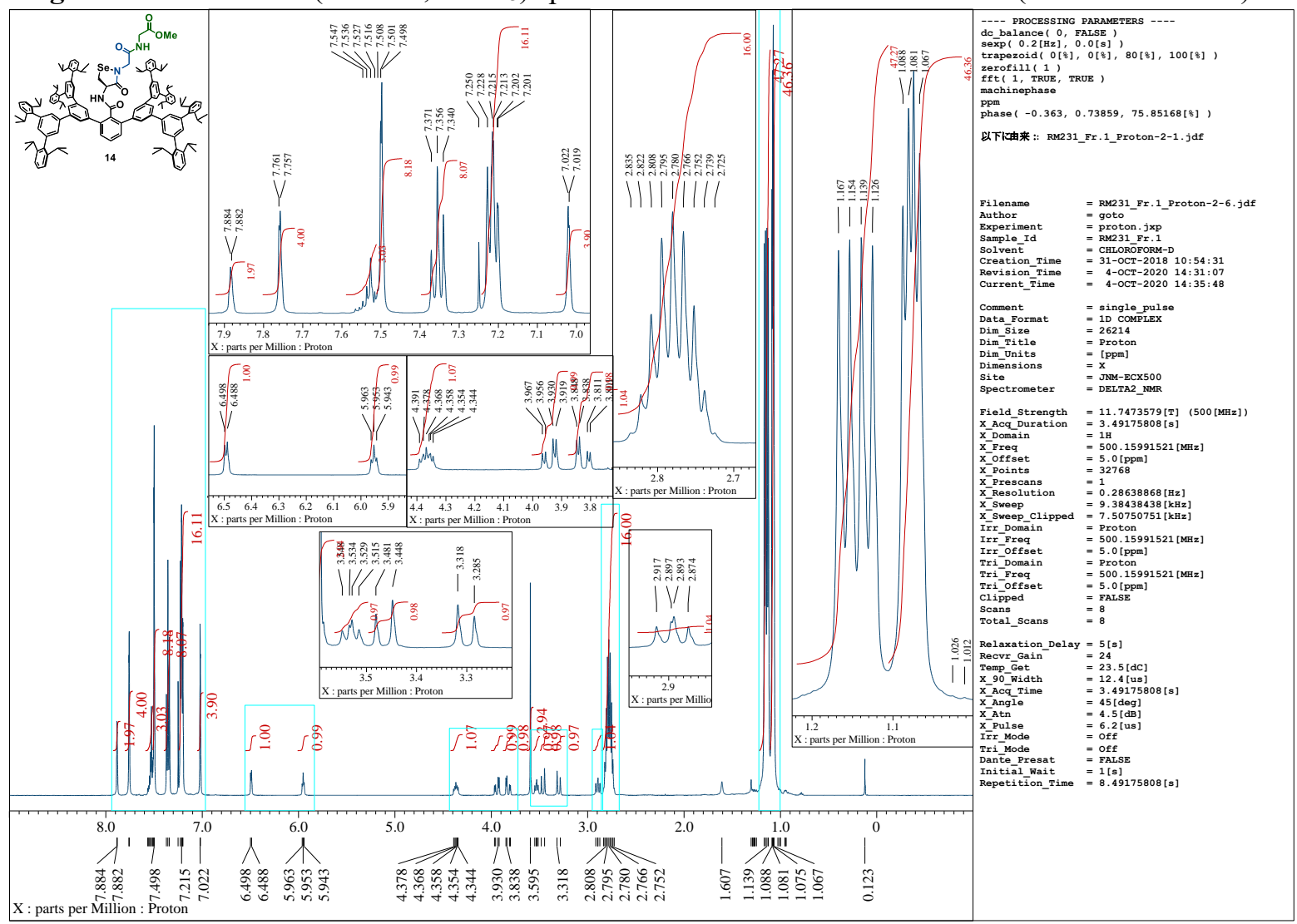

Figure S72. ${ }^{1} \mathrm{H}$ NMR $\left(500 \mathrm{MHz}, \mathrm{CDCl}_{3}\right)$ spectrum of 14. 


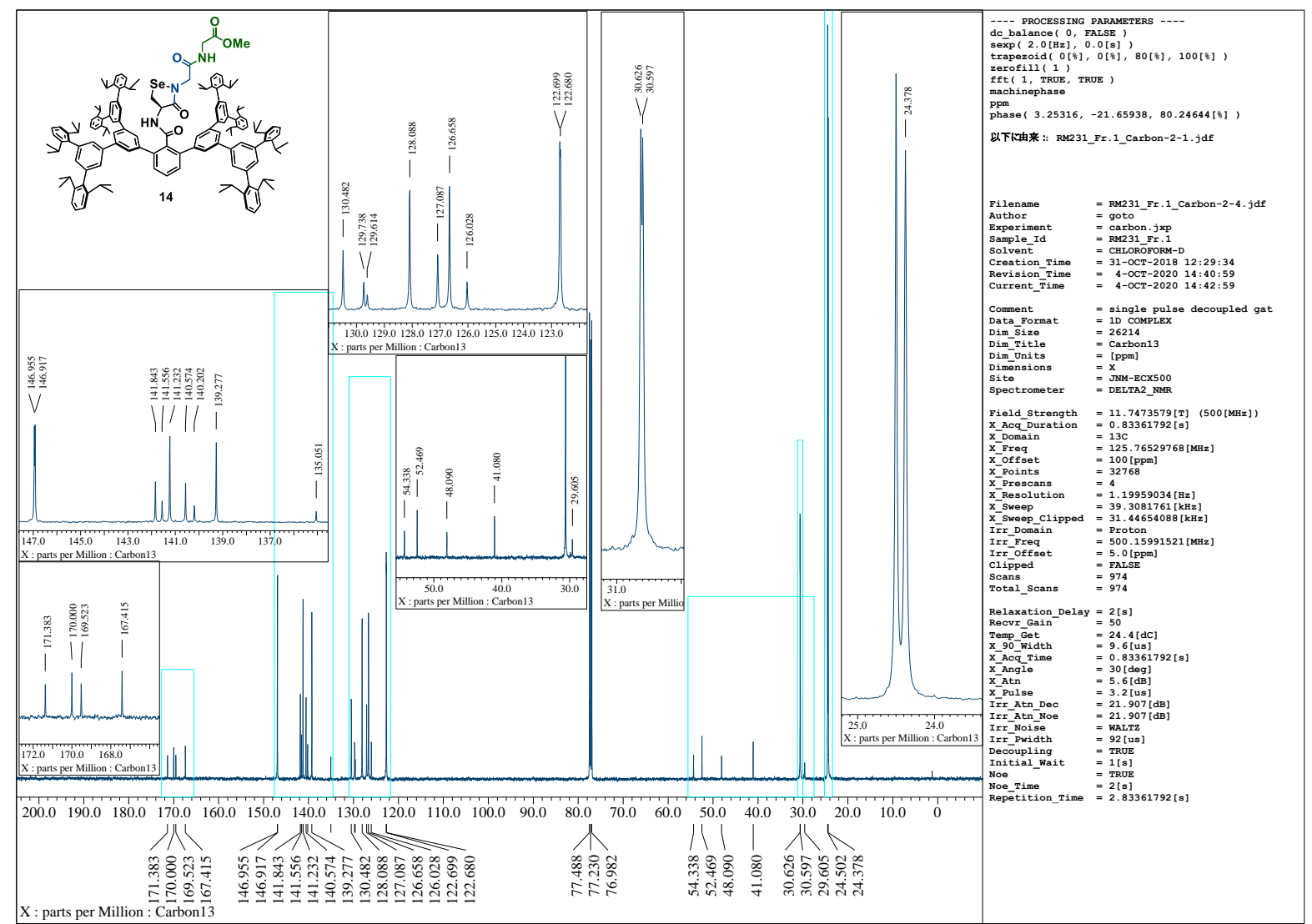

Figure S73. ${ }^{13} \mathrm{C}$ NMR $\left(125 \mathrm{MHz}, \mathrm{CDCl}_{3}\right)$ spectrum of 14.

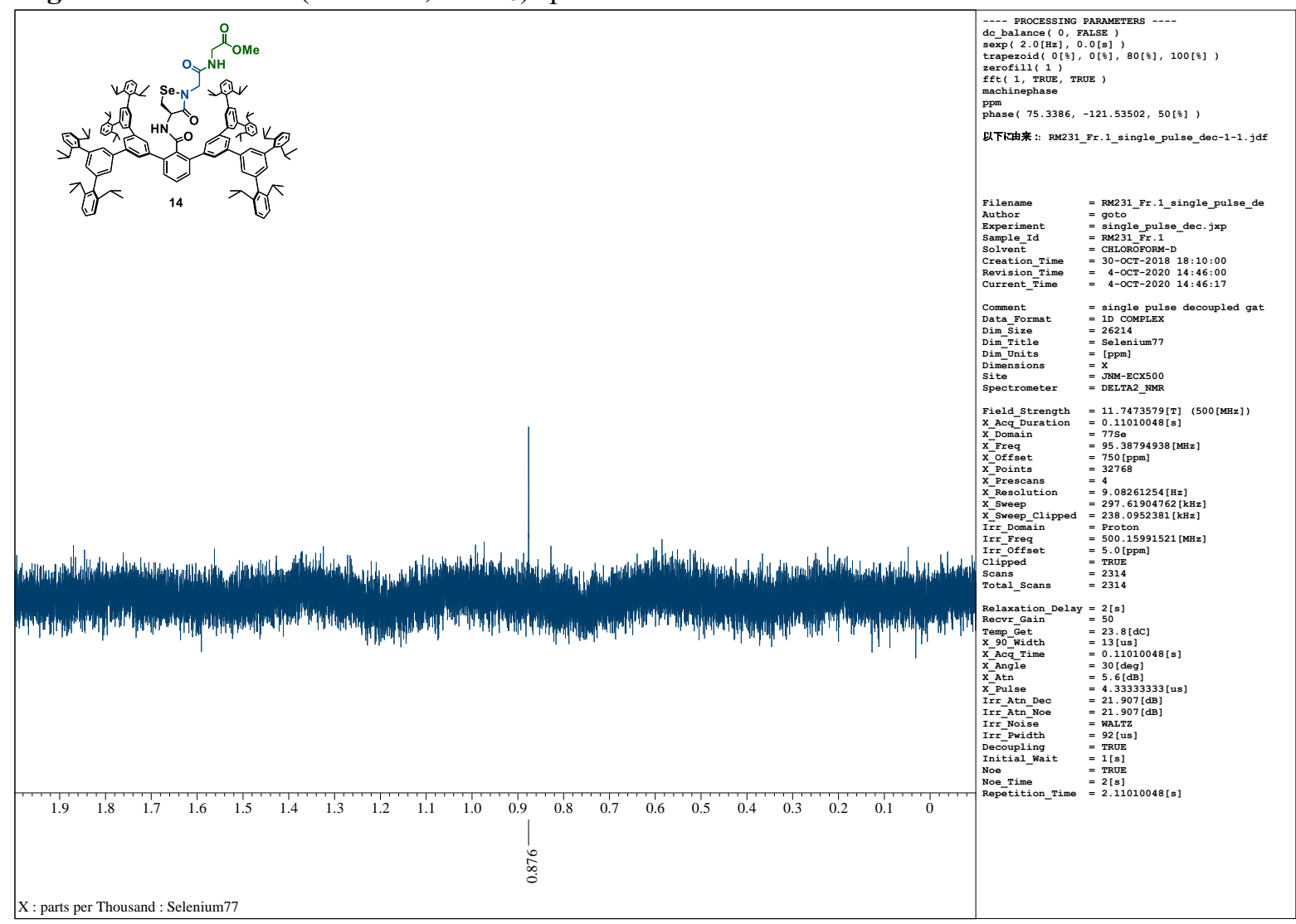

Figure S74. ${ }^{77} \mathrm{Se} \mathrm{NMR}\left(95 \mathrm{MHz}, \mathrm{CDCl}_{3}\right)$ spectrum of 14. 


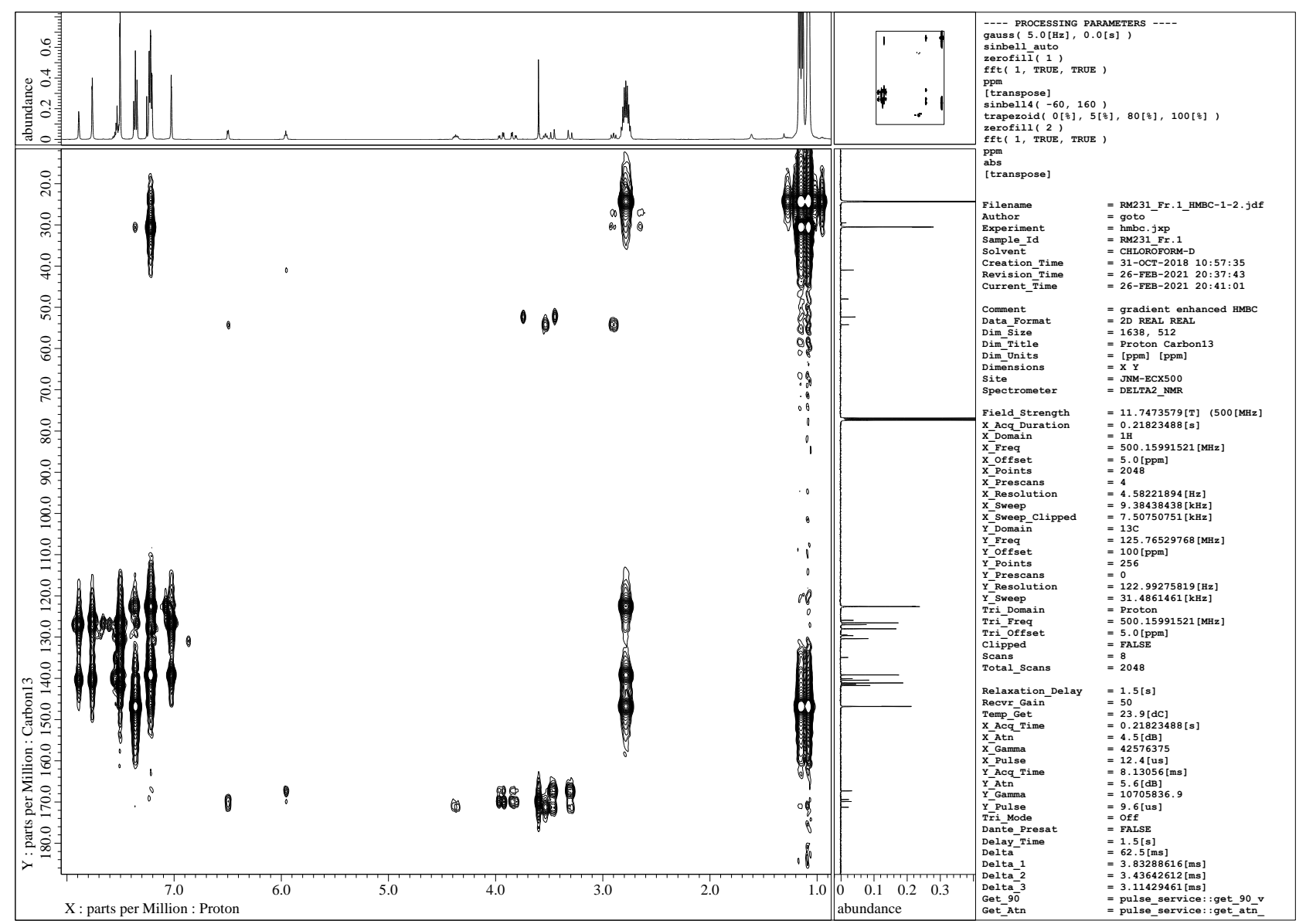

Figure S75. ${ }^{1} \mathrm{H}_{-}{ }^{13} \mathrm{C}$ HMBC (X: $500 \mathrm{MHz}, \mathrm{Y}: 125 \mathrm{MHz}, \mathrm{CDCl}_{3}$ ) spectrum of 14.

\section{References}

1 Sase, S.; Kimura, R.; Masuda, R.; Goto, K. New J. Chem. 2019, 43, 6830-6833.

2 Sano, T.; Masuda, R.; Sase, S.; Goto K. Chem. Commun. 2021, 57, 2479-2482.

3 Stocking, E. -M.; Schwarz, J. -N.; Senn, H.; Salzmann, M.; Silks, L. -A. J. Chem. Soc., Perkin Trans. 1 1997, 2443-2447.

4 Stempel, E.; Kaml, R. F. X.; Budisa, N.; Kalesse, M. Bioorg. Med. Chem. 2018, 26, 5259-5269.

5 Park, H. S.; Kim, C.; Park, M. S. Arch. Pharm. Res. 2015, 38, 791-800.

6 Shimodaira, S.; Iwaoka, M. Phosphorus, Sulfur Silicon Relat. Elem. 2019, 194, 750-752.

7 Dalton, N.; Gordon, C. P.; Boyle, T. P.; Vandegraaf, N.; Deadman, J.; Rhodes, D. I.; Coates, J. A.; Pyne, S. G.; Keller, P. A.; Bremner, J. B. Org. Biomol. Chem. 2016, 14, 6010-6023.

8 Sase, S.; Kakimoto, R.; Goto, K. Angew. Chem., Int. Ed. 2015, 54, 901-904.

9 Ishii, A.; Matsubayashi, S.; Takahashi, T.; Nakayama, J. J. Org. Chem. 1999, 64, 1084-1085.

10 Goto, K.; Nagahama, M.; Mizushima, T.; Shimada, K.; Kawashima, T.; Okazaki, R. Org. Lett. 2001, 3, 35693572 .

11 Saiki, T.; Goto, K.; Okazaki, R. Angew. Chem. Int. Ed. Engl. 1997, 36, 2223-2224.

12 Reich, H. J.; Willis, W. W.; Wollowitz, S. Tetrahedron Lett. 1982, 23, 3319-3322.

13 Reich, H. J.; Jasperse, C. P. J. Org. Chem. 1988, 53, 2389-2390.

14 Iwaoka, M.; Tomoda, S. J. Am. Chem. Soc. 1994, 116, 2557-2561.

15 Goto, K.; Sonoda, D.; Shimada, K.; Sase, S.; Kawashima, T. Angew., Chem., Int. Ed. 2010, 49, $545-547$.

16 Mugesh, G.; Panda, A.; Singh, H. B.; Butcher, R. J. Chem. Eur. J. 1999, 5, 1411-1421. 